\title{
Analysis of D0 -> K+ pi- pi0 Decays: Search for D0-D0bar Mixing, and Measurements of the Doubly Cabibbo-Suppressed Decay Rate and Resonance Contributions
}

\author{
M. Wilson \\ Stanford Linear Accelerator Center \\ Stanford University \\ Stanford, CA 94309
}

SLAC-Report-792

Prepared for the Department of Energy

under contract number DE-AC02-76SF00515

Printed in the United States of America. Available from the National Technical Information Service, U.S.

Department of Commerce, 5285 Port Royal Road, Springfield, VA 22161. 
This document, and the material and data contained therein, was developed under sponsorship of the United States Government. Neither the United States nor the Department of Energy, nor the Leland Stanford Junior University, nor their employees, nor their respective contractors, subcontractors, or their employees, makes an warranty, express or implied, or assumes any liability of responsibility for accuracy, completeness or usefulness of any information, apparatus, product or process disclosed, or represents that its use will not infringe privately owned rights. Mention of any product, its manufacturer, or suppliers shall not, nor is it intended to, imply approval, disapproval, or fitness of any particular use. A royalty-free, nonexclusive right to use and disseminate same of whatsoever, is expressly reserved to the United States and the University. 
UNIVERSITY OF CALIFORNIA

SANTA CRUZ

ANALYSIS OF $D^{0} \rightarrow K^{+} \pi^{-} \pi^{0}$ DECAYS:

SEARCH FOR $D^{0}-\bar{D}^{0}$ MIXING, AND MEASUREMENTS

OF THE DOUBLY CABIBBO-SUPPRESSED DECAY RATE AND RESONANCE CONTRIBUTIONS

A dissertation submitted in partial satisfaction of the requirements for the degree of

DOCTOR OF PHILOSOPHY

in

PHYSICS

by

\section{Michael Galante Wilson}

December 2005

The Dissertation of Michael Galante Wilson is approved:

Professor Abraham Seiden, Chair

Professor Michael Dine

Professor Bruce Schumm

Lisa C. Sloan

Vice Provost and Dean of Graduate Studies 
Copyright (c) by

Michael Galante Wilson

2005 


\section{Contents}

List of Figures $\quad$ vi

\begin{tabular}{ll} 
List of Tables & $\mathbf{x}$ \\
\hline
\end{tabular}

$\begin{array}{ll}\text { Abstract } & \text { xi }\end{array}$

Dedication $\quad$ xiii

Acknowledgments $\quad$ xiv

1 The Physics of $D^{0}-\bar{D}^{0}$ Mixing 1

1.1 Introduction to $D^{0}-\bar{D}^{0}$ Mixing . . . . . . . . . . . . . . . 1

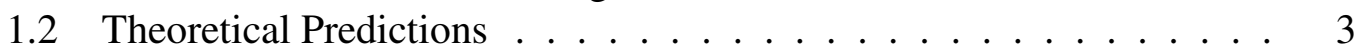

1.3 Current Experimental Status . . . . . . . . . . . . . 8

2 Motivation and Strategy for Analyzing $D^{0} \rightarrow K^{+} \pi^{-} \pi^{0}$ Decays 10

2.1 Searching for $D^{0}-\bar{D}^{0}$ Mixing with a Lifetime Analysis . . . . . . . . 10

2.2 Motivation for Analyzing $D^{0} \rightarrow K^{+} \pi^{-} \pi^{0}$ Decays . . . . . . . . . . 15

2.3 Lifetime Analysis of a Multi-Body $D^{0}$ Decay . . . . . . . . . . . . . 18

2.4 An Analysis Strategy . . . . . . . . . . . . . . . . . 20

3 The BABAR Detector 21

3.1 Track Reconstruction . . . . . . . . . . . . . . . . . 21

3.1.1 Silicon Vertex Tracker . . . . . . . . . . . . 22

3.1 .2 Drift Chamber . . . . . . . . . . . . . 25

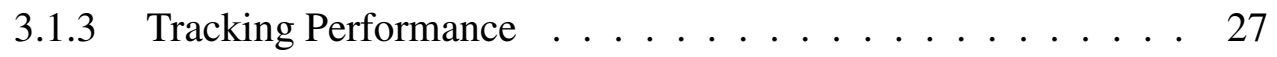

3.2 Particle Identification . . . . . . . . . . . . . . . . . . . 28

3.2.1 $\mathrm{d} E / \mathrm{d} x$ in the Tracking System . . . . . . . . . . . . . . 30

3.2.2 Detector of Internally-Reflected Cherenkov Light . . . . . . . 30 


\subsection{Reconstruction of $\pi^{0}$}

in the Electromagnetic Calorimeter . . . . . . . . . . . . . . . 33

4 Event Selection 36

4.1 Overview of the Selection Process . . . . . . . . . . . . . 36

4.2 Skim Selection . . . . . . . . . . . . . . . . . . 38

4.3 Reskim Selection . . . . . . . . . . . . . . . . 46

5 Dalitz-Plot Efficiency 52

6 Maximum Likelihood Fit to $m_{K \pi \pi^{0}}$ and $\Delta m$ Distributions 58

6.1 Introduction . . . . . . . . . . . . . . . 58

6.2 Descriptions of Fit Categories and Probability Density Functions . . . 60

$6.2 .1 \quad$ Signal . . . . . . . . . . . . . . . . 60

6.2 .2 Non-Peaking Background . . . . . . . . . . . . . 63

$6.2 .3 m_{K \pi \pi^{0}}$ Peaking Background . . . . . . . . . . . . 64

$6.2 .4 \Delta m$ Peaking Background $\ldots \ldots \ldots \ldots \ldots$

6.3 Fit Results . . . . . . . . . . . . . . . . . . . . . . 66

7 Event-Level Flavor Tagging $\quad 74$

7.1 Motivation for a Second Flavor Tag _ . . . . . . . . . . . . 74

$7.2 \pi_{\mathrm{opp}}^{\mp}$ Tag Selection . . . . . . . . . . . . . . . . 76

$7.3 \quad K_{\mathrm{opp}}^{ \pm}$Tag Selection $\ldots \ldots \ldots \ldots \ldots \ldots \ldots \ldots \ldots$

$7.4 e_{\mathrm{opp}}^{\mp} \mathrm{Tag}$ Selection $\ldots \ldots \ldots \ldots \ldots \ldots \ldots$

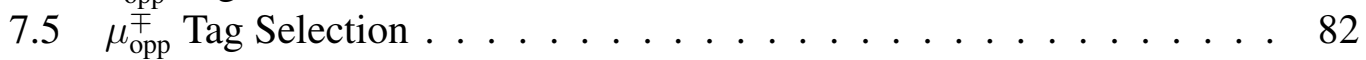

7.6 Tag Efficiencies . . . . . . . . . . . . . . . 83

8 Measurement of $D^{0} \rightarrow K^{+} \pi^{-} \pi^{0}$ Branching Ratio 89

8.1 Dalitz-Plot Efficiency Correction . . . . . . . . . . . . . . 90

8.2 Systematic Uncertainty Estimation . . . . . . . . . . . . . . . . . 91

8.3 Comparison with the Tagged Data Set . . . . . . . . . . . . . 94

8.4 Final Branching Ratio Result . . . . . . . . . . . . . . . . . 95

9 Measurements of Resonance Contributions to $D^{0} \rightarrow K^{+} \pi^{-} \pi^{0} \quad 96$

9.1 Introduction . . . . . . . . . . . . . . 96

9.2 Efficiency-Corrected Two-Body Mass Distributions . . . . . . . . . . . . . . . . . . . . 99

9.3 Description of Probability Density Functions . . . . . . . . . 100

9.4 Fit Results and Systematic Uncertainty Estimation . . . . . . . . . . 104

9.5 Final Resonance-Contribution Results . . . . . . . . . . . . . . 114 
10 Analysis of the $D^{0}$ Decay-Time Distribution 116

10.1 Introduction . . . . . . . . . . . . . . . . 116

10.2 Measured Decay-Time Uncertainties . . . . . . . . . . . . . . . 117

10.3 Description of Right-Sign Probability Density Functions . . . . . . . 119

10.3.1 Signal . . . . . . . . . . . . . . . 119

10.3.2 Combinatoric Background . . . . . . . . . . . . 120

10.3.3 Bad- $D^{*+}$ Background . . . . . . . . . . . . . 121

10.3.4 Bad- $D^{0}$ Background . . . . . . . . . . . . 121

10.4 Right-Sign Fit Results

and $D^{0}$ Lifetime Measurement . . . . . . . . . . . . . . 122

10.5 Selection of Dalitz-Plot Regions . . . . . . . . . . . . . . . . 125

10.6 Description of Wrong-Sign Signal Probability Density Function . . . 128

10.6.1 Motivation and Definition of a Functional Form . . . . . . . . 128

10.6.2 Validation of the Functional Form . . . . . . . . . . . . . 132

10.7 Wrong-Sign Fit Results

and D-Mixing Measurement . . . . . . . . . . . . . 140

10.8 Conclusions . . . . . . . . . . . . . . . . . . . . 149

A Standard Candidate Lists 150

A.1 General Track Lists . . . . . . . . . . . . . . . . . . . . . . 150

A.1.1 GoodTracksVeryLoose . . . . . . . . . . . . 150

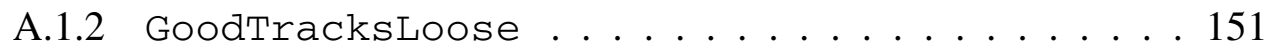

A.2 General Neutral Lists . . . . . . . . . . . . . . . . . . . . . 151

A.2.1 GoodPhotonLoose ................ 151

A.2.2 pioveryLoose. . . . . . . . . . . . . . 152

A.3 Particle Identification Lists . . . . . . . . . . . . . 152

A.3.1 KLHTight . . . . . . . . . . . . . . 152

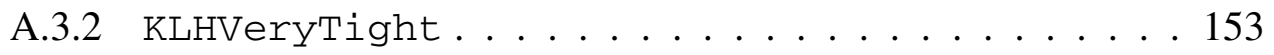

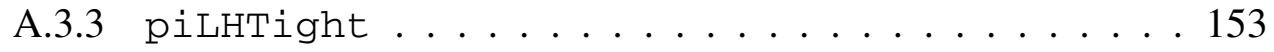

A.3.4 pilHVeryTight . . . . . . . . . . . 153

A.3.5 eMicroloose .................... 154

A.3.6 PidLHElectrons . . . . . . . . . . . . . 154

$\begin{array}{ll}\text { Bibliography } & 168\end{array}$ 


\section{List of Figures}

1.1 Standard-Model quark-level box diagrams contributing to $D^{0}$ mixing . 4

1.2 Hadron-level diagram of a long-distance contribution to mixing . . . . 5

2.1 Quark-level diagrams of $D^{0} \rightarrow K^{+} \pi^{-} \pi^{0}$ and $D^{0} \rightarrow K^{-} \pi^{+} \pi^{0}$ decays 16

3.1 Schematic view of the SVT . . . . . . . . . . . . . 23

3.2 SVT hit resolutions . . . . . . . . . . . . . . . . . 24

3.3 SVT track parameter resolutions and $\Delta z$ measurement errors . . . . . 25

3.4 Schematic view of the DCH ................. 26

3.5 Measurement of $\mathrm{d} E / \mathrm{d} x$ in the $\mathrm{DCH} \ldots \ldots . \ldots 27$

3.6 Low momentum tracking efficiency . . . . . . . . . . . . . . 29

3.7 Fitted track parameter resolutions . . . . . . . . . . . . . . . 29

3.8 Schematic view of the DIRC . . . . . . . . . . . . . . 31

$3.9 D^{0} \rightarrow K \pi$ invariant mass with and without the DIRC . . . . . . . 32

3.10 Schematic view of the EMC . . . . . . . . . . . . . . . . 33

3.11 Energy resolution of the EMC . . . . . . . . . . . . . . . . 35

3.12 Angular resolution of the EMC . . . . . . . . . . . 35

4.1 EMC cluster lateral moment . . . . . . . . . . . . . . . . 41

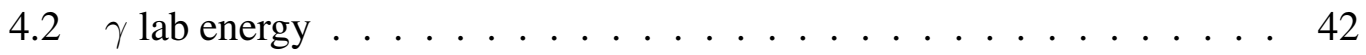

$4.3 \pi^{0}$ mass $m_{\gamma \gamma} \ldots \ldots \ldots \ldots \ldots \ldots \ldots$

$4.4 \pi^{0}$ mass-fit $p\left(\chi^{2}\right) \ldots \ldots \ldots \ldots . \ldots \ldots$

$4.5 D^{0}$ center-of-mass momentum $p^{*} \ldots \ldots \ldots$. . . . . . . . 44

$4.6 D^{0}$ vertex-fit $p\left(\chi^{2}\right) \ldots \ldots \ldots \ldots . \ldots \ldots$

$4.7 \pi^{0}$ lab momentum . . . . . . . . . . . . . . . . 50

4.8 decay chain fit $p\left(\chi^{2}\right) \ldots \ldots \ldots \ldots$. . . . . . . . . 51

5.1 Number of generated signal events in Dalitz-plot bins . . . . . . . . 55

5.2 Efficiency of reconstructing $D^{*+}$ over the Dalitz plot . . . . . . 55 
5.3 Efficiency of reconstructing $D^{*+}$ projected onto $m^{2}$ axes $\ldots . . . .56$

5.4 Pull $\left( \pm \sqrt{\chi^{2}}\right)$ distributions of the binned efficiency fit . . . . . . 56

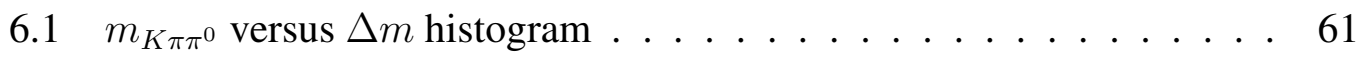

6.2 Illustration of signal PDF components . . . . . . . . . . . . 61

6.3 Projected right-sign fit to data onto $m_{K \pi \pi^{0}}$ and $\Delta m \ldots \ldots$. . . . . 68

6.4 Projected right-sign fit to data onto $m_{K \pi \pi^{0}}$ and $\Delta m$, signal region . . . 68

6.5 Projected right-sign fit to data onto $m_{K \pi \pi^{0}}$, far-sideband region . . . . 69

6.6 Projected right-sign fit to data onto $m_{K \pi \pi^{0}}$, near-sideband region . . . 69

6.7 Projected right-sign fit to data onto $\Delta m$, far-sideband region . . . . . 70

6.8 Projected right-sign fit to data onto $\Delta m$, near-sideband region . . . . 70

6.9 Projected wrong-sign fit to data onto $m_{K \pi \pi^{0}}$ and $\Delta m \ldots \ldots$

6.10 Projected wrong-sign fit data onto $m_{K \pi \pi^{0}}$ and $\Delta m$, signal region . . . 71

6.11 Projected wrong-sign fit to data onto $m_{K \pi \pi^{0}}$, far-sideband region . . . 72

6.12 Projected wrong-sign fit to data onto $m_{K \pi \pi^{0}}$, near-sideband region . . 72

6.13 Projected wrong-sign fit to data onto $\Delta m$, far-sideband region . . . . 73

6.14 Projected wrong-sign fit to data onto $\Delta m$, near-sideband region . . . 73

7.1 Angular difference between $\vec{p}_{\text {est }}$ and $\vec{p}_{\text {true }} \ldots \ldots \ldots \ldots$

$7.2 \pi_{\text {opp }}^{\mp} p_{\perp}$ in $D_{\text {est. }}^{* \mp}$ Rest Frame . . . . . . . . . . . . . . . . . 79

$7.3 \pi_{\text {opp }}^{\mp} p_{\|}$in $D_{\text {est. }}^{* \mp}$ Rest Frame . . . . . . . . . . . . . . . . . 80

$7.4 \pi_{\mathrm{opp}}^{\mp} p$ in $D_{\mathrm{est} \text {. }}^{* \mp}$ Rest Frame . . . . . . . . . . . . . . . . . . . . . 81

$7.5 K_{\mathrm{opp}}^{ \pm}$center-of-mass momentum $p_{K} \ldots \ldots \ldots \ldots . \ldots . \ldots . \ldots 2$

$7.6 e_{\mathrm{opp}}^{\mp}$ center-of-mass momentum $p_{e} \ldots \ldots \ldots \ldots$

$7.7 e_{\mathrm{opp}}^{\mp}$ center-of-mass momentum $p_{\mu} \ldots \ldots \ldots \ldots$. . . . . . . 84

7.8 wrong-sign fit projected onto $m_{K \pi \pi^{0}}$ axis before and after event tag . 88

7.9 wrong-sign fit projected onto $\Delta m$ axis before and after event tag . . . 88

9.1 Dalitz plot of the $D^{0} \rightarrow K^{-} \pi^{+} \pi^{0}$ decay . . . . . . . . . . . . . . 101

$9.2 m^{2}\left(K \pi^{0}\right)$ distributions of efficiency-corrected signal weights . . . . . 101

$9.3 m^{2}(K \pi)$ distributions of efficiency-corrected signal weights . . . . . 102

$9.4 m^{2}\left(\pi \pi^{0}\right)$ distributions of efficiency-corrected signal weights . . . . . 102

9.5 Projection of fit to DCS decays onto $m\left(K^{+} \pi^{0}\right) \ldots \ldots$. . . . . . . 111

9.6 Projection of fit to CF decays onto $m\left(K^{-} \pi^{0}\right) \ldots \ldots . . . . . .111$

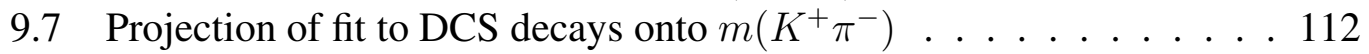

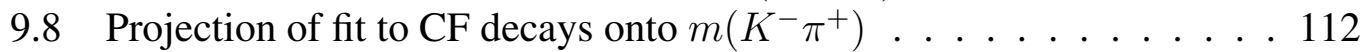

9.9 Projection of fit to DCS decays onto $m\left(\pi^{-} \pi^{0}\right) \ldots \ldots . \ldots 113$

9.10 Projection of fit to CF decays onto $m\left(\pi^{+} \pi^{0}\right) \ldots \ldots$. . . . . . . 113

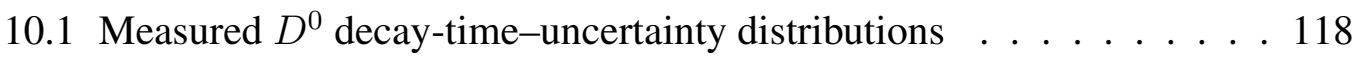

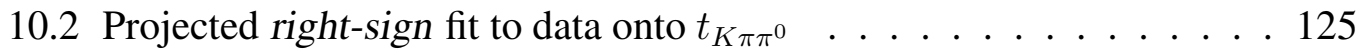


10.3 Projected right-sign fit to data onto $t_{K \pi \pi^{0}}$ in signal regions $\ldots \ldots 126$

10.4 Projected right-sign fit to data onto $t_{K \pi \pi^{0}}$ in sideband regions of both

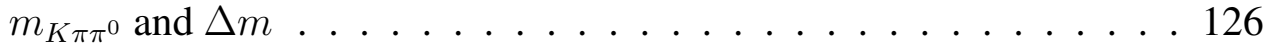

10.5 Projected right-sign fit to data onto $t_{K \pi \pi^{0}}$ in sideband region of $\Delta m$ in the $m_{K \pi \pi^{0}}$ signal region . . . . . . . . . . . . . . . 127

10.6 Projected right-sign fit to data onto $t_{K \pi \pi^{0}}$ in sideband regions of $m_{K \pi \pi^{0}}$ in the $\Delta m$ signal region . . . . . . . . . . . . . . . . . . . 127

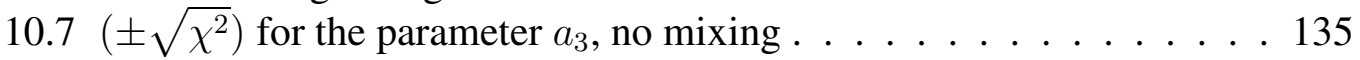

$10.8\left( \pm \sqrt{\chi^{2}}\right)$ for the parameter $a_{3}$, large interference term . . . . 135

$10.9\left( \pm \sqrt{\chi^{2}}\right)$ for the parameter $a_{3}$, moderate interference term . . . . 136

$10.10\left( \pm \sqrt{\chi^{2}}\right)$ for the parameter $a_{3}$, small interference term . . . . . . 136

10.11 Likelihood contours for Monte Carlo events with no mixing . . . . . 137

10.12Likelihood contours for Monte Carlo events with mixing, positive interference . . . . . . . . . . . . . . . . . 138

10.13Likelihood contours for Monte Carlo events with mixing, negative interference . . . . . . . . . . . . . . . . . . 139

10.14Likelihood contours for final data set . . . . . . . . . . . . . . 144

10.15Projected wrong-sign fit to data onto $t_{K \pi \pi^{0}} \ldots \ldots \ldots \ldots \ldots$

10.16Projected wrong-sign fit to data onto $t_{K \pi \pi^{0}}$ in signal regions $\ldots . .145$

10.17Projected wrong-sign fit to data onto $t_{K \pi \pi^{0}}$ in sideband regions of both $m_{K \pi \pi^{0}}$ and $\Delta m \ldots \ldots \ldots \ldots \ldots \ldots \ldots$

10.18Projected wrong-sign fit to data onto $t_{K \pi \pi^{0}}$ in sideband region of $\Delta m$ in the $m_{K \pi \pi^{0}}$ signal region . . . . . . . . . . . . . . 146

10.19Projected wrong-sign fit to data onto $t_{K \pi \pi^{0}}$ in sideband regions of $m_{K \pi \pi^{0}}$ in the $\Delta m$ signal region . . . . . . . . . . . . . 147

10.20Projected wrong-sign fit to data onto $t_{K \pi \pi^{0}}$, null-hypothesis . . . . 148

A.1 efficiency of $K$ comparing Monte Carlo and data in KLHTight . . 156

A.2 efficiency of $K$ as a function of $p$ in KLHTight . . . . . . . . 156

A.3 efficiency of $K$ as a function of $\theta$ in KLHTight . . . . . . . 157

A.4 efficiency of $\pi$ comparing Monte Carlo and data in KLHTight . . . 157

A.5 efficiency of $\pi$ as a function of $p$ in KLHTight . . . . . . . . 158

A.6 efficiency of $\pi$ as a function of $\theta$ in KLHTight . . . . . . . 158

A.7 efficiency of $K$ comparing Monte Carlo and data in KLHVeryTight 159

A.8 efficiency of $K$ as a function of $p$ in KLHVeryTight . . . . . . . 159

A.9 efficiency of $K$ as a function of $\theta$ in KLHVeryTight . . . . . . 160

A.10 efficiency of $\pi$ comparing Monte Carlo and data in KLHVeryTight 160

A.11 efficiency of $\pi$ as a function of $p$ in KLHVeryTight . . . . . . . 161

A.12 efficiency of $\pi$ as a function of $\theta$ in KLHVeryTight . . . . . . . 161

A.13 efficiency of $\pi$ comparing Monte Carlo and data in piLHTight . . 162 
A.14 efficiency of $\pi$ as a function of $p$ in piLHTight . . . . . . . 162

A.15 efficiency of $\pi$ as a function of $\theta$ in piLHTight . . . . . . . 163

A.16 efficiency of $K$ comparing Monte Carlo and data in piLHTight $\ldots 163$

A.17 efficiency of $K$ as a function of $p$ in piLHTight . . . . . . . . . 164

A.18 efficiency of $K$ as a function of $\theta$ in piLHTight . . . . . . . . 164

A.19 efficiency of $\pi$ comparing Monte Carlo and data in piLHVeryTight 165

A.20 efficiency of $\pi$ as a function of $p$ in piLHVeryTight . . . . . 165

A.21 efficiency of $\pi$ as a function of $\theta$ in piLHVeryTight . . . . . 166

A.22 efficiency of $K$ comparing Monte Carlo and data in piLHVeryTight 166

A.23 efficiency of $K$ as a function of $p$ in piLHVeryTight . . . . . 167

A.24 efficiency of $K$ as a function of $\theta$ in piLHVeryTight . . . . . 167 


\section{List of Tables}

4.1 Definitions of skim variables . . . . . . . . . . . . . . . 40

4.2 Estimated signal efficiency for skimming . . . . . . . . . . 41

4.3 Definitions of reskim variables . . . . . . . . . . . . . 47

4.4 Estimated signal efficiency for reskimming $\ldots \ldots \ldots \ldots$

6.1 Results of fit to data . . . . . . . . . . . . . . . . . . 67

7.1 Definitions of $\pi_{\mathrm{opp}}^{\mp}$ variables $\ldots \ldots \ldots \ldots \ldots \ldots \ldots$

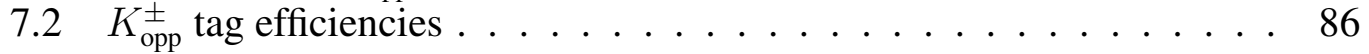

$7.3 \pi_{\mathrm{opp}}^{\mp}$ tag efficiencies $\ldots \ldots \ldots \ldots \ldots \ldots \ldots \ldots \ldots$

7.4 combined tag efficiencies $\ldots \ldots \ldots \ldots \ldots$

8.1 Branching ratio systematic uncertainties $\ldots \ldots \ldots$. . . . . 93

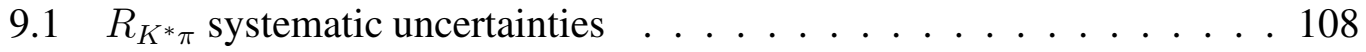

$9.2 R_{K^{* 0} \pi^{0}}$ systematic uncertainties $\ldots \ldots \ldots \ldots$

$9.3 R_{K \rho}$ systematic uncertainties . . . . . . . . . . . . 108

$9.4 \mathcal{B} r\left(D^{0} \rightarrow K^{*-} \pi^{+}\right)$systematic uncertainties . . . . . . . . . . 109

$9.5 \mathcal{B} r\left(D^{0} \rightarrow \bar{K}^{* 0} \pi^{0}\right)$ systematic uncertainties . . . . . . . . . . . . . 109

$9.6 \mathcal{B} r\left(D^{0} \rightarrow K^{-} \rho^{+}\right)$systematic uncertainties . . . . . . . . . . 110

9.7 DCS-to-CF branching ratios . . . . . . . . . . . . . 115

9.8 Branching ratios of DCS resonances to $D^{0} \rightarrow K^{+} \pi^{-} \pi^{0} \ldots \ldots \ldots 115$

9.9 Branching ratios of $\mathrm{CF}$ resonances to $D^{0} \rightarrow K^{-} \pi^{+} \pi^{0} \ldots \ldots \ldots 115$

10.1 Decay-time-uncertainty scale factors from the likelihood fit . . . . . 123

10.2 Parameters used for generating wrong-sign Monte Carlo events . . . . 133

10.3 Fitted values of mixing parameters . . . . . . . . . . . . 141

10.4 Values of physical quantities, determined by the fit . . . . . . . 141

10.5 Monte Carlo data sets demonstrating the no-mixing consistency $\ldots 142$ 


\author{
Abstract \\ Analysis of $\boldsymbol{D}^{0} \rightarrow \mathrm{K}^{+} \boldsymbol{\pi}^{-} \boldsymbol{\pi}^{0}$ Decays: \\ Search for $D^{0}-\bar{D}^{0}$ Mixing, \\ and Measurements \\ of the Doubly Cabibbo-Suppressed Decay Rate \\ and Resonance Contributions
}

by

Michael Galante Wilson

Analyzing $D^{0} \rightarrow K^{+} \pi^{-} \pi^{0}$ decays, herein are presented the methods and results of a search for $D^{0}-\bar{D}^{0}$ mixing, a measurement of the branching ratio $R \equiv$ $\Gamma\left(D^{0} \rightarrow K^{+} \pi^{-} \pi^{0}\right) / \Gamma\left(D^{0} \rightarrow K^{-} \pi^{+} \pi^{0}\right)$, and measurements of the contributions from $D^{0} \rightarrow K^{+} \rho^{-}, K^{*+} \pi^{-}, K^{* 0} \pi^{0} ; 230.4 \mathrm{fb}^{-1}$ of data collected from the BABAR detector at the PEP-II collider during 2000-2004 (Runs 1-4) are analyzed. An eventlevel tagging technique is developed, which facilitates the accurate determination of doubly Cabibbo-suppressed resonance contributions by suppressing background from Cabibbo-favored decays. The branching ratio is measured as $R=(0.214 \pm$ 0.008 (stat) \pm 0.008 (syst) $) \%$, with $(46.1 \pm 3.3$ (stat) \pm 2.9 (syst) $) \%$ of $D^{0} \rightarrow K^{+} \pi^{-} \pi^{0}$ decays proceeding through the channel $D^{0} \rightarrow K^{*+} \pi^{-}$. The data are consistent with the null-D-mixing hypothesis at a confidence level of $10 \%$, and the expected value 
of $\pm \sqrt{x^{2}+y^{2}}$ is measured as $-0.013 \pm 0.010$ (stat), indicating negative interference between mixing and doubly Cabibbo-suppressed decay. The expected value of the integrated mixing rate is $\left(x^{2}+y^{2}\right) / 2=(0.013 \pm 0.013$ (stat) $) \%$. 
This is dedicated to my brilliant wife, Christian. 


\section{Acknowledgments}

This work was supported in part by the U.S. Department of Energy under contract numbers DE-FG02-04ER41286 and DE-AC02-76SF00515.

I am grateful to all of my colleagues in the BABAR Collaboration who contributed to the construction, operation, maintenance, and understanding of the world-class particle detector with which the data studied in this work were recorded. In particular, I would like to acknowledge the charm-analysis working group for dialogue and review that helped to develop this analysis. My collaborators have always shown themselves to be scientists of the highest caliber.

The size of the data sample is due to the extraordinary contributions of our PEP-II colleagues in achieving excellent luminosity and machine conditions. The success of this project also relies critically on the expertise and dedication of the computing organizations that support $B A B A R$.

The extension of the $D$-mixing formalism to multi-body decays and the compelling motivation for studying $D^{0} \rightarrow K^{+} \pi^{-} \pi^{0}$ decays were clearly articulated to me by Abraham Seiden, who has truly been a mentor and advisor. I am grateful also for all of the efforts of my Reading Committee.

The technical and stylistic quality of this work are due in large part to the rigorous and outstanding editing of my colleague Christian Flacco. Her consistent and methodical approach to Physics yielded countless improvements in the details of this 
analysis.

Finally, I would like to thank my family, particularly my parents, Carmella and Stephen Wilson, and my brother, Paul Wilson, for their continued support throughout my life, which has given me the opportunities to pursue my whimsies. 


\section{Chapter 1}

\section{The Physics of $D^{0}-\bar{D}^{0}$ Mixing}

\subsection{Introduction to $D^{0}-\bar{D}^{0}$ Mixing}

$D^{0}-\bar{D}^{0}$ mixing is the quantum-mechanical oscillation between the two eigenstates $\left|D^{0}\right\rangle$ and $\left|\bar{D}^{0}\right\rangle$ of the Strong and Electromagnetic Interactions. Mixing occurs because these eigenstates are not identical to the eigenstates of the full Standard-Model Hamiltonian. Specifically, these states are not eigenstates of the Weak Interaction. The time evolution of this system is described by the Schrödinger equation

$$
i \frac{\partial}{\partial t}\left(\begin{array}{c}
D^{0}(t) \\
\bar{D}^{0}(t)
\end{array}\right)=\left(\mathbf{M}-\frac{i}{2} \boldsymbol{\Gamma}\right)\left(\begin{array}{c}
D^{0}(t) \\
\bar{D}^{0}(t)
\end{array}\right),
$$


where $M$ and $\Gamma$ are Hermitian matrices describing mixing and decay, respectively. Expressing the mass eigenstates in terms of the above eigenstates,

$$
\left|D_{1,2}\right\rangle=p\left|D^{0}\right\rangle \pm q\left|\bar{D}^{0}\right\rangle,
$$

where $p$ and $q$ are complex numbers, we can diagonalize the Hamiltonian and calculate the eigenvalues [1]. If $C P$ is a conserved quantity in this system, then $p=q$. The two mass eigenstates have different masses $\left(m_{1}, m_{2}\right)$ and decay widths $\left(\Gamma_{1}, \Gamma_{2}\right)$ generated by the mixing dynamics, and we parameterize the mixing process with the quantities $x$ and $y$ defined in terms of the differences in mass and width:

$$
x \equiv 2 \frac{m_{2}-m_{1}}{\Gamma_{2}+\Gamma_{1}}, \quad y \equiv \frac{\Gamma_{2}-\Gamma_{1}}{\Gamma_{2}+\Gamma_{1}} .
$$

There is an ambiguity at the time of writing because it is not known which state, $\left|D_{1}\right\rangle$ or $\left|D_{2}\right\rangle$, is the more massive state. When a convention is needed, we identify $\left|D_{1,2}\right\rangle=\left|D_{L, H}\right\rangle$ so that $x$ is positive by construction. Most of the formalism and results presented herein are independent of this particular convention, and it is noted when this convention is applied.

$C P$ violation might be observed in an experimental search for $D^{0}-\bar{D}^{0}$ mixing. As in other cases, such as the $B^{0}-\bar{B}^{0}$ or $K^{0}-\bar{K}^{0}$ systems, it could appear in any of three ways. The first way would be a modification of the mixing rate because $p \neq q$ in Equation 1.2 above. The second and third ways would be modifications of either the decay rates or the interference between mixing and decay for the particular decay 
channels chosen in an analysis. As explained below, an observation of $C P$ violation would be the most suggestive sign of new physics in this system.

\subsection{Theoretical Predictions}

In the Standard Model, the mixing rate in the $D^{0}-\bar{D}^{0}$ system is expected on general grounds to be very small, but it is difficult to calculate how small because of significant contributions from long-distance effects. This theoretical uncertainty limits, but does not eliminate, the potential for identifying signs of new physics.

At small distances, neutral-meson mixing proceeds via flavor-changing neutral currents (FCNC). Since there are no tree-level FCNC contributions in the Standard Model, processes such as mixing occur at the quark level primarily via box diagrams, as shown in Figure 1.1. In the $B^{0}-\bar{B}^{0}$ or $K^{0}-\bar{K}^{0}$ systems, the mesons comprise downtype quarks. In contrast, the $D^{0}-\bar{D}^{0}$ system comprises up-type quarks. $D$ mixing is therefore sensitive to the contributions of virtual down-type quarks coupled to the Weak Interaction, making it a process that might reveal physics not seen in the $B$ or $K$ systems. One immediate result of this difference is that there is no contribution from heavy quarks in the $D$-mixing box diagram. This particular type of contribution breaks the Glashow-Iliopoulos-Maiani (GIM) cancellation in the $B$ or $K$ systems $^{1}$ and allows sizable mixing rates. The heaviest quark in the $D$-mixing box diagram is the

\footnotetext{
${ }^{1}$ In $K$ mixing, it is $m_{c}^{2}-m_{u}^{2}$ that is relevant; in $B$ mixing, it is $m_{t}^{2}-m_{c}^{2}$.
} 

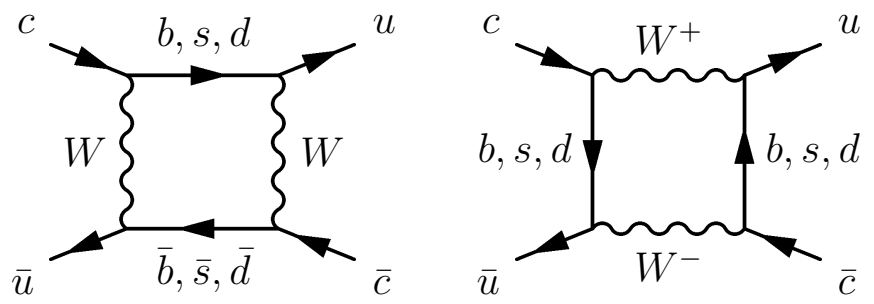

Figure 1.1: Standard-Model box diagrams of flavor-changing neutral currents contributing to $D^{0}-\bar{D}^{0}$ mixing at the quark level

b-quark. Because the suppression from Cabibbo-Kobayashi-Maskawa (CKM) mixing factors $V_{c b} V_{u b}^{*}$ outweighs the potential contribution of the $b$-quark in the box, the $b$ quark does not significantly affect the $D$-mixing rate [2]. Thus, the short-distance contribution to $x$ comes primarily from transitions to $d$ - and $s$-quarks, and this is estimated to be [1]

$$
x_{\text {box }} \approx \mathcal{O}\left(10^{-6}\right)-\mathcal{O}\left(10^{-5}\right)
$$

Short-distance contributions to $y$ are further suppressed.

Long-distance contributions to $D$ mixing are therefore expected to be dominant, but these contributions are non-perturbative and cannot be calculated from first principles. They come from transitions to final states $|f\rangle$ that are accessible to both $\left|D^{0}\right\rangle$ and $\left|\bar{D}^{0}\right\rangle$. For example, Figure 1.2 illustrates a contribution to mixing from transitions to two pseudoscalars. It has long been observed that $D$ mixing is an effect of flavor$\mathrm{SU}(3)\left(\mathrm{SU}(3)_{F}\right)$ breaking $[3,4]$, and there has been a continuing effort to estimate the size of this symmetry breaking. It has also been shown that if $\mathrm{SU}(3)_{F}$ breaking is 


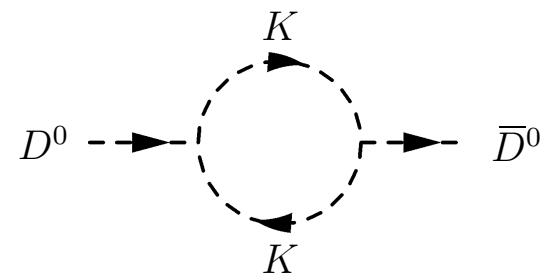

Figure 1.2: A hadron-level diagram of a long-distance physics contribution to $D^{0}-\bar{D}^{0}$ mixing

perturbative, it is at most a second-order effect [5]:

$$
x, y \approx \sin ^{2} \theta_{c} \times\left[\mathrm{SU}(3)_{F} \text { breaking }\right]^{2} .
$$

If one parameterizes all $\mathrm{SU}(3)_{F}$ breaking with the $s$-quark mass $m_{s}$, then one can estimate model independently

$$
x, y \approx \sin ^{2} \theta_{c} \times\left(\frac{m_{s}}{\Lambda_{\text {hadr. }}}\right)^{2} \lesssim \mathcal{O}\left(10^{-3}\right),
$$

where $\Lambda_{\text {hadr. }} \approx \mathcal{O}(1) \mathrm{GeV}$ is a typical hadronic scale. Beyond this simple estimate, there are two main approaches to estimating the long-distance contributions to mixing: an inclusive approach using an operator product expansion (OPE), and an exclusive approach that sums over intermediate hadronic states using experimental data. Neither approach is completely satisfactory, and the resulting estimates of $y$ differ by an order of magnitude.

The inclusive approach applies Heavy-Quark Effective Theory (HQET) to calculate contributions to $D$ mixing, an approach first taken by Georgi [6] and later ex- 
tended by others $[7,8]$. There are two main assumptions. The first is that the mass of the $c$-quark is large, $m_{c} \gg \Lambda_{\text {hadr. }}$. The second is that one can construct local quarklevel operators that can be applied to hadron-level processes (quark-hadron duality is applicable). This leads to series expansions of $x$ and $y$ in terms of local operators with powers of $\left(\Lambda / m_{c}\right)$. The result of this type of approach is [8]

$$
x \approx y \approx \mathcal{O}\left(10^{-3}\right) .
$$

The exclusive approach takes all of the known hadronic states common to both $\left|D^{0}\right\rangle$ and $\left|\bar{D}^{0}\right\rangle$, and groups them both according to their respective $\mathrm{SU}(3)_{F}$ multiplets and to the number of particles in the final state. An example of such a set would be $\left\{\pi^{+} \pi^{-}, \pi^{+} K^{-}, K^{+} K^{-}, K^{+} \pi^{-}\right\}$. In the limit of a perfect $\mathrm{SU}(3)_{F}$ symmetry, the individual contributions within each of these groups would cancel, and there would be no mixing. If one knows the relative amplitudes and strong phases for these states, calculations of $x$ and $y$ can be done for each multiplet. For the example set above, this calculation gives a small contribution due to cancellations, a reasonable result since all of the states in the set are far from threshold and not affected as much by phase space considerations. Contributions to $x$ are not required to be on-shell, so in this case there is no symmetry breaking caused by limited phase space. If one assumes that all of the sets contribute incoherently in roughly the same amount, one concludes that [1]

$$
x \lesssim \mathcal{O}\left(10^{-3}\right) .
$$


By contrast, contributions to $y$ are due to on-shell states, so phase space is a significant source of symmetry breaking. Considering phase space as the only source of symmetry breaking, one can calculate the contribution to $y$ of each of the final-state multiplets for which there is data using only the measured masses of the final-state particles [5]. The largest calculable contribution comes from the final-state multiplet comprising four pseudoscalars, whose elements are either near the production threshold with relatively large branching fractions, or are above threshold and entirely absent. This method concludes that [5]

$$
y \lesssim \mathcal{O}\left(10^{-2}\right)
$$

This is a good estimate if other sources of symmetry breaking do not cancel the effects of limited phase space. However, quark-hadron duality, one of the critical assumptions of the inclusive method, naturally produces such cancellations.

Traditionally, it has been argued that a measurement of $x$ or $y \gtrsim \mathcal{O}\left(10^{-2}\right)$ would be a sign of new physics, but the exclusive calculation outlined above calls this argument into question. It has also been shown that any $C P$ violation coming from new physics would be suppressed and most likely unobservable if $y \gg x$ [9]. Despite these limitations, the possibility of observing new physics remains. A measurement of $x \gg y$ would still be suggestive of new physics even for large values of $y$. Because $D$ mixing only involves the first two quark generations, $C P$ violation from StandardModel sources is very small on general grounds. Observing $C P$ violation in a search 
for $D$ mixing would be a sign of new physics. Finally, in the absence of a clear signal for new physics, it appears that the measured rate of $D$ mixing could at least shed light on the applicability of quark-hadron duality in the charm sector.

\subsection{Current Experimental Status}

$D^{0}-\bar{D}^{0}$ mixing has not been observed at the time of writing, but many experiments have conducted searches using a variety of techniques. The most stringent limits on $D$ mixing have been set by the the BABAR, Belle, and CLEO collaborations, analyzing hadronic $D^{0}$ decays.

The current benchmark for conducting a $D$ mixing search is an analysis of the decay-time distribution of $D^{0} \rightarrow K^{+} \pi^{-}$decays. BABAR [10], Belle [11], and CLEO [12] have all performed such analyses. The advantages of this analysis strategy are, first, a clean signal from the relatively narrow $\left(m_{D^{*+}}-m_{D^{0}}\right)$ peak; and second, the ability to compare the signal distributions to the high statistics $D^{0} \rightarrow K^{-} \pi^{+}$sample, eliminating many sources of systematic bias. A disadvantage is that there is no sensitivity to $x$ or $y$ individually, but only to $x^{2}+y^{2}$ and $y_{K \pi}^{\prime}$, where $y_{K \pi}^{\prime}$ is related to $y$ by an unknown strong phase. (It may be possible in the future to determine the strong phase associated with $y_{K \pi}^{\prime}$ at CLEO-c [13] by taking advantage of the quantum coherence of $\psi(3770)$ at production.)

Another analysis strategy involves measuring the ratio of lifetimes for $D^{0}$ de- 
cays into states of specific $C P$. This technique has been used by E791 [14] and FOCUS [15], and improved upon by BABAR [16] to include possible $C P$ violating effects. These analyses are only sensitive to $y$.

Finally, one can analyze semileptonic $D^{0}$ decays to search for mixing. E791 [17] and $B A B A R$ [18] have used this technique, among others. Unlike the hadronic lifetime analyses, the semileptonic lifetime analysis does not have to contend with a possible interference term between mixing and a doubly Cabibbo-suppressed decay. However, it has worse signal resolution because of the unreconstructed neutrino in the event.

The combined world $95 \%$-confidence limit on the time-integrated $D$-mixing rate is

$$
\left(\frac{x^{2}+y^{2}}{2}\right)<0.13 \%
$$




\section{Chapter 2}

\section{Motivation and Strategy for}

\section{Analyzing $D^{0} \rightarrow K^{+} \pi^{-} \pi^{0}$ Decays}

\subsection{Searching for $D^{0}-\bar{D}^{0}$ Mixing with a Lifetime Analysis}

A search for mixing attempts to identify the process ${ }^{1}\left|D^{0}\right\rangle \rightarrow\left|\bar{D}^{0}\right\rangle$ by analyzing the decay products of a particle known to be created as a $\left|D^{0}\right\rangle$. In practice, this means reconstructing the state $|\bar{f}\rangle$ in an attempt to observe

$$
\left|D^{0}\right\rangle \rightarrow\left|\bar{D}^{0}\right\rangle \rightarrow|\bar{f}\rangle
$$

\footnotetext{
${ }^{1}$ Charge conjugation is implied except where otherwise stated.
} 
The difficulty comes from the fact that for hadronic (i.e. nonleptonic) systems, the decay

$$
\left|D^{0}\right\rangle \rightarrow|\bar{f}\rangle
$$

can occur directly, without any mixing at all. Distinguishing Process 2.1 from 2.2 is the primary goal of this analysis, and it relies on the fact that the decay-time distribution of the final state $|\bar{f}\rangle$ is different for the two processes. The most sensitivity to mixing will be found when the amplitude for Process 2.2 is as small as possible, and therefore doubly Cabibbo-suppressed (DCS) decays are chosen for this type of analysis. DCS decays have very small branching fractions, on the order of

$$
\left|\sin ^{2}\left(\theta_{C}\right)\right|^{2} \approx 0.0025
$$

times the corresponding Cabibbo-favored (CF) decay branching fraction, where $\theta_{C}$ is the Cabibbo angle. To the extent that Process 2.2 has a smaller amplitude for a particular decay compared to others, it will yield more sensitivity to a sign of mixing.

Following from Equation 1.1, and ignoring the possibility of $C P$ violation (forbidden in the Standard Model), one can derive the time-dependent equations:

$$
\begin{aligned}
\left|D^{0}(t)\right\rangle & =a_{+}(t)\left|D^{0}\right\rangle+a_{-}(t)\left|\bar{D}^{0}\right\rangle \\
\left|\bar{D}^{0}(t)\right\rangle & =a_{-}(t)\left|D^{0}\right\rangle+a_{+}(t)\left|\bar{D}^{0}\right\rangle
\end{aligned}
$$


where

$$
\begin{aligned}
& a_{+}(t)=\frac{1}{2}\left(e^{-i m_{1} t-\frac{1}{2} \Gamma_{1} t}+e^{-i m_{2} t-\frac{1}{2} \Gamma_{2} t}\right) \\
& a_{-}(t)=\frac{1}{2}\left(e^{-i m_{1} t-\frac{1}{2} \Gamma_{1} t}-e^{-i m_{2} t-\frac{1}{2} \Gamma_{2} t}\right) .
\end{aligned}
$$

We are interested in the measurable, time-dependent rate:

$$
\begin{aligned}
\Gamma_{\bar{f}}(t)= & \left|\left\langle\bar{f}|\mathcal{H}| D^{0}(t)\right\rangle\right|^{2} \\
= & \left(\left|A_{\bar{f}}\right|^{2}\left|a_{+}(t)\right|^{2}+A_{\bar{f}} \bar{A}_{\bar{f}}^{*} a_{+}(t) a_{-}^{*}(t)\right. \\
& \left.+\bar{A}_{\bar{f}} A_{\bar{f}}^{*} a_{-}(t) a_{+}^{*}(t)+\left|\bar{A}_{\bar{f}}\right|^{2}\left|a_{-}(t)\right|^{2}\right),
\end{aligned}
$$

where

$$
\begin{aligned}
& A_{\bar{f}}=\left\langle\bar{f}|\mathcal{H}| D^{0}\right\rangle \\
& \bar{A}_{\bar{f}}=\left\langle\bar{f}|\mathcal{H}| \bar{D}^{0}\right\rangle .
\end{aligned}
$$

In general, there is a phase difference between $A_{\bar{f}}$ and $\bar{A}_{\bar{f}}$ arising from the Strong Interaction. To understand this, consider the set of strong eigenstates $|n\rangle$ that can scatter into the final state $|\bar{f}\rangle$. Similar to Equations 2.9-2.10, we can write the amplitudes for decay into these eigenstates:

$$
\begin{aligned}
& A_{n}=\left\langle n|\mathcal{H}| D^{0}\right\rangle=b_{n} e^{i \delta_{n}} \\
& \bar{A}_{n}=\left\langle n|\mathcal{H}| \bar{D}^{0}\right\rangle=\bar{b}_{n} e^{i \delta_{n}},
\end{aligned}
$$


where $b_{n}, \bar{b}_{n}$ are real numbers. In terms of these eigenstates,

$$
\begin{aligned}
& A_{\bar{f}}=\sum_{n}\langle\bar{f} \mid n\rangle\left\langle n|\mathcal{H}| D^{0}\right\rangle=\sum_{n} c_{n} b_{n} e^{i \delta_{n}} \\
& \bar{A}_{\bar{f}}=\sum_{n}\langle\bar{f} \mid n\rangle\left\langle n|\mathcal{H}| \bar{D}^{0}\right\rangle=\sum_{n} c_{n} \bar{b}_{n} e^{i \delta_{n}} .
\end{aligned}
$$

In the general case,

$$
\begin{gathered}
b_{n}=k_{n} \bar{b}_{n} \\
k_{n} \neq k_{m} \text { for } n \neq m,
\end{gathered}
$$

where $k_{n}$ are constants. Unless there is a unique $k$ such that $k_{n}=k$ for all $n$, then there is a phase shift between $A_{\bar{f}}$ and $\bar{A}_{\bar{f}}$. Therefore, we can write

$$
A_{\bar{f}} \bar{A}_{\bar{f}}^{*}=\left|A_{\bar{f}}\right|\left|\bar{A}_{\bar{f}}\right| e^{-i \delta}
$$

where $\delta$ is the strong phase difference. We can simplify Equation 2.8 under the assumptions

$$
\left|A_{\bar{f}}\right| \ll\left|\bar{A}_{\bar{f}}\right|, \quad|x|,|y| \ll 1
$$


and expressing

$$
\begin{aligned}
& \left|A_{\bar{f}}\right|^{2}\left|a_{+}(t)\right|^{2}=\left|A_{\bar{f}}\right|^{2} \frac{e^{-\Gamma t}}{2}[\cosh (y \Gamma t)+\cos (x \Gamma t)] \\
& \approx\left|A_{\bar{f}}\right|^{2} e^{-\Gamma t} \\
& \left|\bar{A}_{\bar{f}}\right|^{2}\left|a_{-}(t)\right|^{2}=\left|\bar{A}_{\bar{f}}\right|^{2} \frac{e^{-\Gamma t}}{2}[\cosh (y \Gamma t)-\cos (x \Gamma t)] \\
& \quad \approx\left|\bar{A}_{\bar{f}}\right|^{2} e^{-\Gamma t}\left(\frac{x^{2}+y^{2}}{4}\right)(\Gamma t)^{2} \\
& A_{\bar{f}} \bar{A}_{\bar{f}}^{*} a_{+}(t) a_{-}^{*}(t)+\bar{A}_{\bar{f}} A_{\bar{f}}^{*} a_{-}(t) a_{+}^{*}(t) \\
& \quad=\left|A_{\bar{f}}\right|\left|\bar{A}_{\bar{f}}\right| \frac{e^{-\Gamma t}}{2}\left(e^{-i \delta}(\sinh (y \Gamma t)-i \sin (x \Gamma t))+e^{i \delta}(\sinh (y \Gamma t)+i \sin (x \Gamma t))\right) \\
& \quad \approx\left|A_{\bar{f}}\right|\left|\bar{A}_{\bar{f}}\right| e^{-\Gamma t}(y \cos \delta-x \sin \delta)(\Gamma t)
\end{aligned}
$$

where $\Gamma$ is the average decay width of $\Gamma_{1}$ and $\Gamma_{2}$. If we define

$$
\begin{aligned}
R_{D} & \equiv \frac{\left|A_{\bar{f}}\right|^{2}}{\left|\bar{A}_{\bar{f}}\right|^{2}} \\
y^{\prime} & \equiv y \cos \delta-x \sin \delta
\end{aligned}
$$

we obtain the standard form for the time-dependent decay rate, including $D$ mixing:

$$
\Gamma_{\bar{f}}(t)=\left|\bar{A}_{\bar{f}}\right|^{2} e^{-\Gamma t}\left(R_{D}+\sqrt{R_{D}} y^{\prime}(\Gamma t)+\frac{x^{2}+y^{2}}{4}(\Gamma t)^{2}\right) .
$$

As shown, $D$ mixing is characterized in the decay rate by a deviation away from a pure exponential. In order to have the most sensitivity to $\left(x^{2}+y^{2}\right)$, a decay channel 
for which $R_{D}$ is relatively small is desired. The analysis technique benefits from the ability to compare the signal distribution, given by Equation 2.24, to the Cabibbofavored decay distribution, which may be treated as a pure exponential. In this way, systematic bias is significantly limited.

\subsection{Motivation for Analyzing $D^{0} \rightarrow K^{+} \pi^{-} \pi^{0}$ Decays}

In choosing a decay mode with which to study $D$ mixing, one must weigh the advantageous physical properties of the system against the experimental difficulties one will encounter. Hadronic $D^{0}$ decays can be reconstructed relatively easily and cleanly, especially the two-body $D^{0} \rightarrow K^{\mp} \pi^{ \pm}$decays. The analyses of these decays $[10,11,12]$ have set the most stringent limits on the size of $D$ mixing to date. However, these analyses find that for this mode, $R_{D}=(0.362 \pm 0.029) \%$, which is a bit higher than the crude estimate given in Equation 2.3. One might expect that in the limit of flavor-SU(3) symmetry, summing over all doubly Cabibbo-suppressed modes, and the corresponding Cabibbo-favored modes, would yield a value of $R_{D} \approx 0.25 \%$. By this reasoning, it makes sense to search for DCS decays for which $R_{D}<0.25 \%$, for which one might have more sensitivity to a sign of mixing.

The wrong-sign decay $D^{0} \rightarrow K^{+} \pi^{-} \pi^{0}$ is intriguing for a number of reasons. First, the corresponding CF decay $D^{0} \rightarrow K^{-} \pi^{+} \pi^{0}$ has the relatively high branching fraction of $13 \%$. By comparison, the branching fraction for $D^{0} \rightarrow K^{-} \pi^{+}$is $3.8 \%$. 


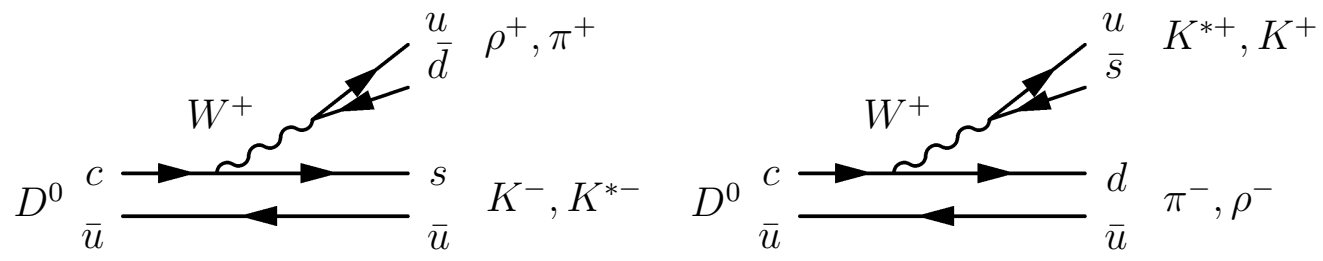

Figure 2.1: Quark-level Feynman diagrams of the Cabibbo-favored (CF) $D^{0} \rightarrow K^{-} \pi^{+} \pi^{0}$ decay (left) and the doubly Cabibbo-suppressed (DCS) $D^{0} \rightarrow K^{+} \pi^{-} \pi^{0}$ decay (right). Possible final-state hadrons have been included to demonstrate how one might expect the DCS Dalitz plot to differ from the CF Dalitz plot. In particular, if one assumes that the $W^{+}$couples more strongly to a spin-1 final-state particle, the main resonant contribution to the DCS decay might be $D^{0} \rightarrow K^{*+} \pi^{-}$.

Thus, one expects to analyze a relatively large sample of wrong-sign decays. Second, we gain sensitivity to mixing if the Dalitz-plot structure of the DCS decay differs from that of the CF decay, and there is good reason to believe that the Dalitz plots are different by considering the relevant Feynman diagrams (see Figure 2.1). The sensitivity may be increased by selecting regions of the Dalitz plot where the CF decay contributes with a larger amplitude relative to the corresponding DCS amplitude, since a mixing signal will have the CF Dalitz-plot structure. Third, the $R_{D}$ value for this mode was poorly known when this analysis was begun [19], and there has since been evidence [20] that $R_{D}=\left(0.229 \pm 0.015_{-0.009}^{+0.013}\right) \%$.

There are significant experimental difficulties with the decay $D^{0} \rightarrow K^{+} \pi^{-} \pi^{0}$ as compared to $D^{0} \rightarrow K^{+} \pi^{-}$, however. All of these additional difficulties are due to reconstruction of the $\pi^{0}$ candidate. First, the efficiency for reconstructing $\pi^{0}$ cancels 
any gain in statistics from the large branching fraction. The net effect is that we reconstruct about as many $D^{0} \rightarrow K^{-} \pi^{+} \pi^{0}$ decays as $D^{0} \rightarrow K^{-} \pi^{+}$decays. Second, the width of the $D^{0}$-mass distribution is $\approx 60 \%$ greater than that of a decay mode comprising only charged tracks. The average measurement uncertainty is rather large, and the shape of the distribution of measurement uncertainties is poorly known. This makes harder the separation of signal from background in a maximum likelihood fit, for which the signal and background shapes must be known precisely. Third, we have found that the resolution of the $D^{0}$ mass is so poor that significant correlations are introduced with the distribution of $\delta m=\left(m_{K \pi \pi^{0} \pi_{s}}-m_{K \pi \pi^{0}}\right)$, which is also used to separate signal from background. Fourth, and finally, the simulation of the BABAR detector does not correctly reproduce the means and resolutions of the mass distributions of particles decaying to photons, such as $\pi^{0}$. Thus, the simulated data cannot be used for detailed determination of the distribution shapes; rather, the parameters specifying these shapes must be fit from data.

Notwithstanding the experimental difficulties that must be overcome, the analysis of $D^{0} \rightarrow K^{+} \pi^{-} \pi^{0}$ decays is compelling. With the same number of reconstructed CF decays as for $D^{0} \rightarrow K^{-} \pi^{+}$, but with a lower $R_{D}$ value and a Dalitz plot to take advantage of, the analysis of $D^{0} \rightarrow K^{+} \pi^{-} \pi^{0}$ decays offers perhaps the best present chance of observing $D$ mixing. 


\subsection{Lifetime Analysis of a Multi-Body $D^{0}$ Decay}

For a multi-body decay, the lifetime analysis is slightly different from what is presented in Section 2.1, in order to account for resonance contributions. Consider a decay for which the Dalitz plot is parameterized by the variables $\left\{m_{12}, m_{23}\right\}$. Equation 2.24 is true for any point in the Dalitz plot. However, it does not hold for a region of the Dalitz plot. Specifically, both the strong-phase difference and the ratio of the number of DCS decays to that of CF decays may vary as functions of $\left\{m_{12}, m_{23}\right\}$. If they do, then the interference term is suppressed.

Starting from Equation 2.8, we note that $A_{\bar{f}}$ and $\bar{A}_{\bar{f}}$ vary as functions of $\left\{m_{12}, m_{23}\right\}$ :

$$
\begin{aligned}
& A_{\bar{f}}=A_{\bar{f}}\left(m_{12}, m_{23}\right) \\
& \bar{A}_{\bar{f}}=\bar{A}_{\bar{f}}\left(m_{12}, m_{23}\right)
\end{aligned}
$$

Because $A_{\bar{f}}$ and $\bar{A}_{\bar{f}}$ are not time-dependent, we may integrate them over any region of the Dalitz plot, for any value of $t$, and Equations 2.19-2.20 remain substantively the same:

$$
\begin{aligned}
& \int\left|A_{\bar{f}}\left(m_{12}, m_{23}\right)\right|^{2}\left|a_{+}(t)\right|^{2} d m_{12} d m_{23}=A_{I}^{2} \frac{e^{-\Gamma t}}{2}[\cosh (y \Gamma t)+\cos (x \Gamma t)] \\
& \int\left|\bar{A}_{\bar{f}}\left(m_{12}, m_{23}\right)\right|^{2}\left|a_{-}(t)\right|^{2} d m_{12} d m_{23}=\bar{A}_{I}^{2} \frac{e^{-\Gamma t}}{2}[\cosh (y \Gamma t)-\cos (x \Gamma t)]
\end{aligned}
$$


However, consider

$$
\begin{aligned}
& \int A_{\bar{f}}\left(m_{12}, m_{23}\right) \bar{A}_{\bar{f}}\left(m_{12}, m_{23}\right)^{*} d m_{12} d m_{23} \\
& \quad=\int\left|A_{\bar{f}}\left(m_{12}, m_{23}\right)\right|\left|\bar{A}_{\bar{f}}\left(m_{12}, m_{23}\right)\right| e^{-i \delta\left(m_{12}, m_{23}\right)} d m_{12} d m_{23} \\
& \quad=\alpha A_{I} \bar{A}_{I} e^{-i \delta_{I}}, \quad 0 \leq \alpha \leq 1
\end{aligned}
$$

The result of integrating the strong-phase difference, which varies as a function of $\left\{m_{12}, m_{23}\right\}$, is to reduce the interference term. Also, to the extent that the ratio of $\left|A_{\bar{f}}\left(m_{12}, m_{23}\right)\right|$ to $\left|\bar{A}_{\bar{f}}\left(m_{12}, m_{23}\right)\right|$ varies, the interference term is further reduced compared to the other two terms in the rate equation (Equation 2.32 below). These reductions are parameterized by the real coefficient $\alpha$. Defining

$$
\begin{aligned}
R_{I} & \equiv \frac{A_{I}^{2}}{\bar{A}_{I}^{2}} \\
y_{I}^{\prime} & \equiv y \cos \delta_{I}-x \sin \delta_{I}
\end{aligned}
$$

we obtain a form for the time-dependent decay rate for a multi-body decay:

$$
\begin{gathered}
\Gamma_{\bar{f}}(t)=\bar{A}_{I}^{2} e^{-\Gamma t}\left(R_{I}+\alpha \sqrt{R_{I}} y_{I}^{\prime}(\Gamma t)+\frac{x^{2}+y^{2}}{4}(\Gamma t)^{2}\right), \\
0 \leq \alpha \leq 1 .
\end{gathered}
$$

It should be noted that while $\alpha, R_{I}$ and $y_{I}^{\prime}$ depend on the Dalitz plot region that has been selected for analysis, the mixing term $\left(x^{2}+y^{2}\right)$ is independent of the Dalitz plot variables, as it is independent of the decay mode chosen for analysis.

If a Dalitz plot region is chosen such that only one resonance contributes in that region, then $\alpha \approx 1$. If a Dalitz plot region is chosen such that several non-overlapping 
resonances contribute, and those resonances contribute the same relative amounts to the overall DCS and CF Dalitz plots, then again $\alpha \approx 1$.

\subsection{An Analysis Strategy}

The analysis of wrong-sign $D^{0} \rightarrow K^{+} \pi^{-} \pi^{0}$ and right-sign $D^{0} \rightarrow K^{-} \pi^{+} \pi^{0}$ decays undertaken in this work proceeds in three sequential steps, with the ultimate goal of searching for a signal of non-zero $\left(x^{2}+y^{2}\right)$. First, we determine the overall branching ratio $\Gamma\left(D^{0} \rightarrow K^{+} \pi^{-} \pi^{0}\right) / \Gamma\left(D^{0} \rightarrow K^{-} \pi^{+} \pi^{0}\right)$ using a maximum likelihood fit to extract the number of signal events in the wrong-sign and right-sign samples. Second, we examine the Dalitz structure of the DCS decays by analyzing the two-body mass projections $m\left(\pi \pi^{0}\right), m\left(K \pi^{0}\right)$, and $m(K \pi)$, fitting for the fraction of signal events with contributions from $K \rho, K^{*} \pi$, and $K^{* 0} \pi^{0}$. With the new knowledge of the population of the DCS Dalitz plot, we conclude with a lifetime analysis on a sample of the data selected to substantially reduce $R_{I}$ in Equation 2.32 and enhance sensitivity to $D$ mixing. 


\section{Chapter 3}

\section{The $B_{A} B_{A R}$ Detector}

The data used in this analysis were collected from the BABAR detector [21], a particle detector operating on the PEP-II asymmetric storage ring at the Stanford Linear Accelerator Center (SLAC). At this facility, an electron beam of $9.0 \mathrm{GeV}$ and a positron beam of $3.1 \mathrm{GeV}$ are collided. The BABAR detector has several component systems; those that contribute to this analysis are discussed in some detail below. For this work, there are three areas of interest: charged-track reconstruction (including at low momentum), charged-particle identification (PID), and $\pi^{0}$ reconstruction.

\subsection{Track Reconstruction}

Charged-track reconstruction (i.e. tracking) in BABAR proceeds through the tandem contributions of its two inner-most sub-systems: the silicon vertex tracker (SVT) 
and the drift chamber $(\mathrm{DCH})$. The SVT is designed to provide both vertexing capabilities and independent tracking of low-momentum charged particles. Through careful alignment and calibration, these two detectors support efficient high-precision tracking in $B A B A R$ over a broad momentum spectrum, detecting charged particles down to transverse momenta of $50 \mathrm{MeV} / c$. The tracking system operates within a $1.5 \mathrm{~T}$ solenoidal magnetic field supplied by superconducting electromagnets; the steel flux return, making up the outermost layer of the BABAR detector, is instrumented to identify muons and detect neutral hadrons.

\subsubsection{Silicon Vertex Tracker}

The physics goals of $B A B A R$ require a single-vertex resolution of $80 \mu \mathrm{m}$. In addition to vertex capabilities, the SVT must also provide stand-alone tracking of charged particles with low transverse momentum $\left(p_{t}<120 \mathrm{MeV} / c\right)$ that will not travel far enough from the beam axis to be accurately measured in the drift chamber.

The physical constraints at the PEP-II interaction region significantly affect the design of the SVT. Permanent dipole magnets are located at a distance of $\pm 20 \mathrm{~cm}$ from the interaction point, so the SVT must be mounted on them. The magnets limit the acceptance angle of the detector to the range $17.2^{\circ}<\theta<150^{\circ}$. To achieve high luminosity, bunches are spaced only $4.2 \mathrm{~ns}$ apart, meaning hit information must be buffered and the readout should be sparse. Finally, the anticipated radiation for the inner-most 


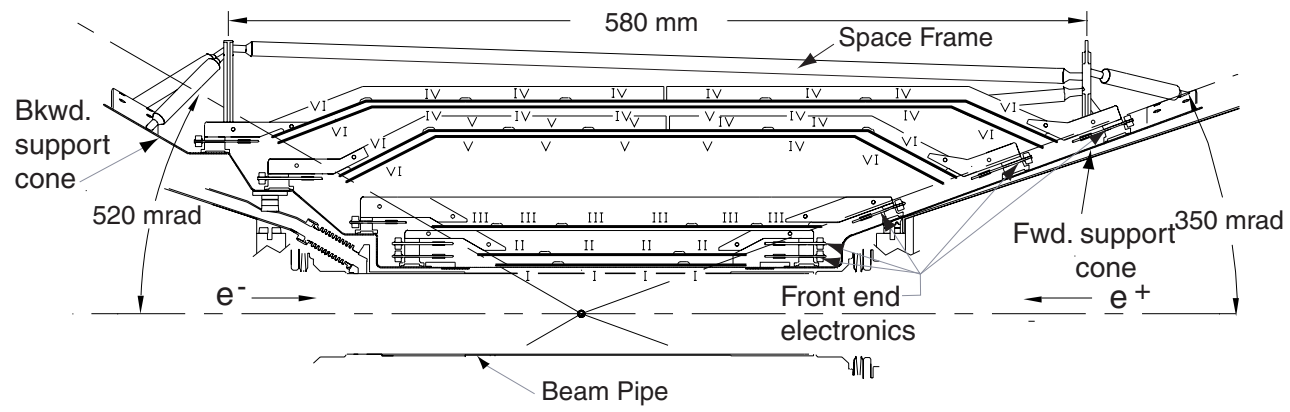

Figure 3.1: Schematic view of the SVT: longitudinal section. The roman numerals label the six different types of sensors.

layers is expected to be $250-400 \mathrm{kRad} / \mathrm{yr}$ on average and $1000-2000 \mathrm{kRad} / \mathrm{yr}$ in the horizontal bending plane of the beams (caused by the permanent dipole magnets), so the detector must be radiation hard.

The SVT consists of 5 layers of double-sided AC-coupled silicon sensors (Figure 3.1). The inner three layers are critical in providing vertex information, and we require a single-hit resolution of $10-15 \mu \mathrm{m}$. These sensors are arranged in a sixsided cylindrical shape. The outer two layers provide tracking information and pattern recognition that can be used with other sub-detectors or in a stand-alone manner. These outer modules are designed in an arc shape in order to minimize the amount of silicon required and to increase the crossing angle for detected particles near the edge of the acceptance region. A single-hit resolution of $30-40 \mu \mathrm{m}$ is sufficient in these modules.

The SVT has met or exceeded all of its performance design goals. Single hit resolution for perpendicular tracks matches the design specifications: $10-15 \mu \mathrm{m}$ for layers 

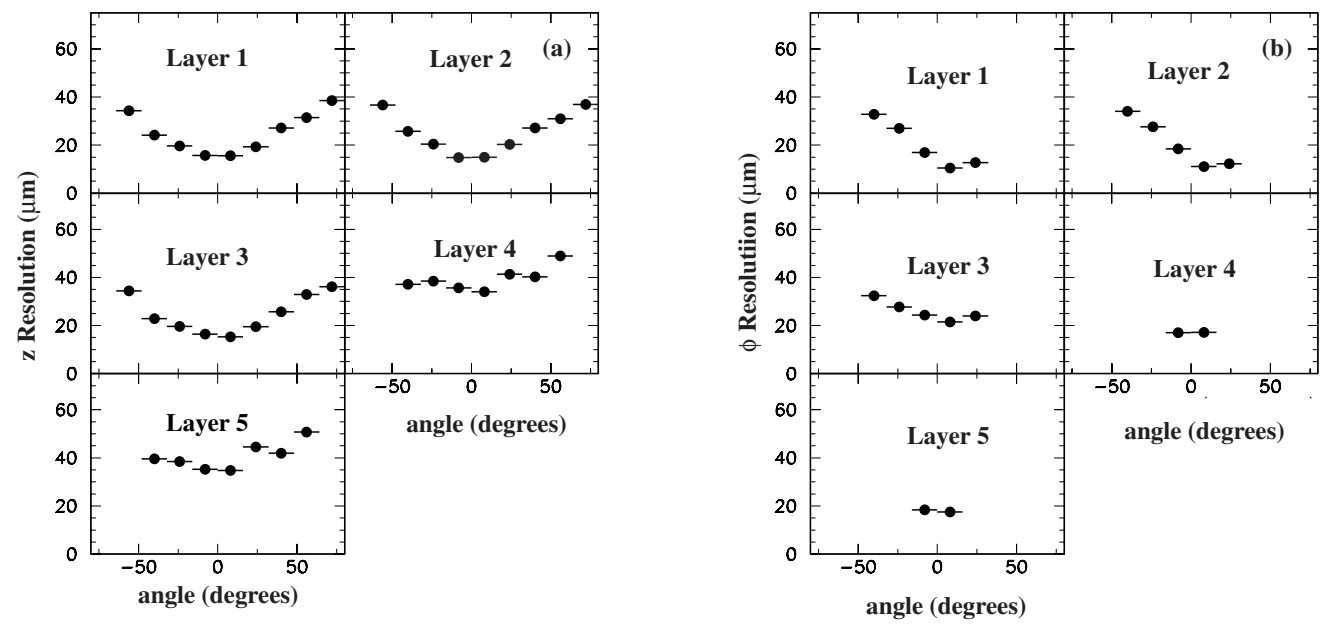

Figure 3.2: SVT hit resolutions as a function of track incidence angle: along the beam axis $z$ (left) and perpendicular to the beam axis $\phi$ (right).

1-3 and 30-40 $\mu \mathrm{m}$ for layers 4-5 (Figure 3.2). Hit reconstruction is typically better than $98 \%$ for all functioning modules. Figure 3.3 (left) shows the resolution of the reconstructed track parameters $d_{0}$ and $z_{0}$, which represent the distances between the point of closest approach to the $z$-axis and the origin in the $x$-y plane and along the $z$ axis, respectively. Figure 3.3 (right) shows the estimated error in the measurement of the difference along the $z$-axis between the vertices of the two neutral $B$ mesons, one of which is fully reconstructed. The SVT provides tracking information for particles with low transverse momentum $p_{t} \gtrsim 50 \mathrm{MeV} / c$, which is important for tracking soft pions, for example from $D^{*+} \rightarrow D^{0} \pi^{+}$. 

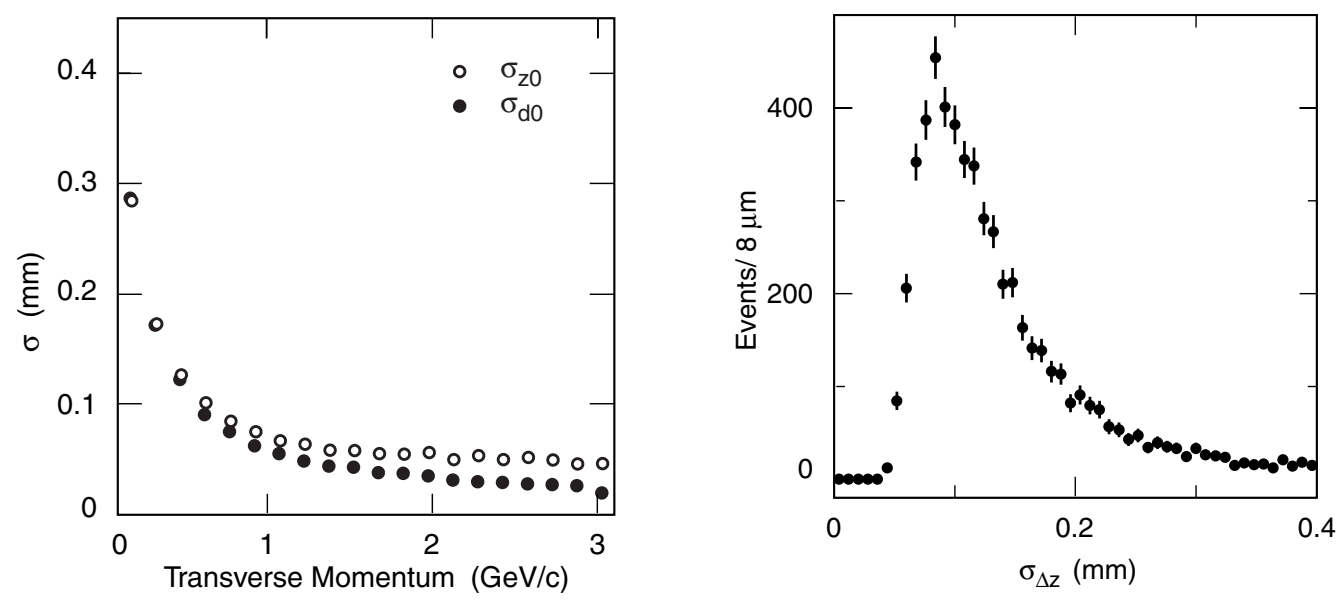

Figure 3.3: (Left) Resolution in the parameters $d_{0}$ and $z_{0}$ for tracks in multi-hadron events as a function of the transverse momentum. (Right) Distribution of the error on the difference $\Delta z$ between $B$ meson vertices, one of which is fully reconstructed.

\subsubsection{Drift Chamber}

The primary role of the drift chamber $(\mathrm{DCH})$ is to provide high-precision momenta measurements for charged particles. This is important for low-background reconstruction of exclusive $D$-meson decays.

The DCH is composed of 40 layers of small hexagonal cells consisting of one sense wire surrounded by six field wires. It is relatively small in diameter, and it has a length of almost $3 \mathrm{~m}$ (Figure 3.4 ). With this geometry, charged particles with transverse momenta above $180 \mathrm{GeV} / c$ may have up to 40 spatial and ionization-loss $(\mathrm{d} E / \mathrm{d} x)$ measurements in this detector. The $\mathrm{DCH}$ also provides longitudinal spacial measurements. This capability is due to the placement of wires in 24 of the 40 layers at small angles relative to the primary axis. 


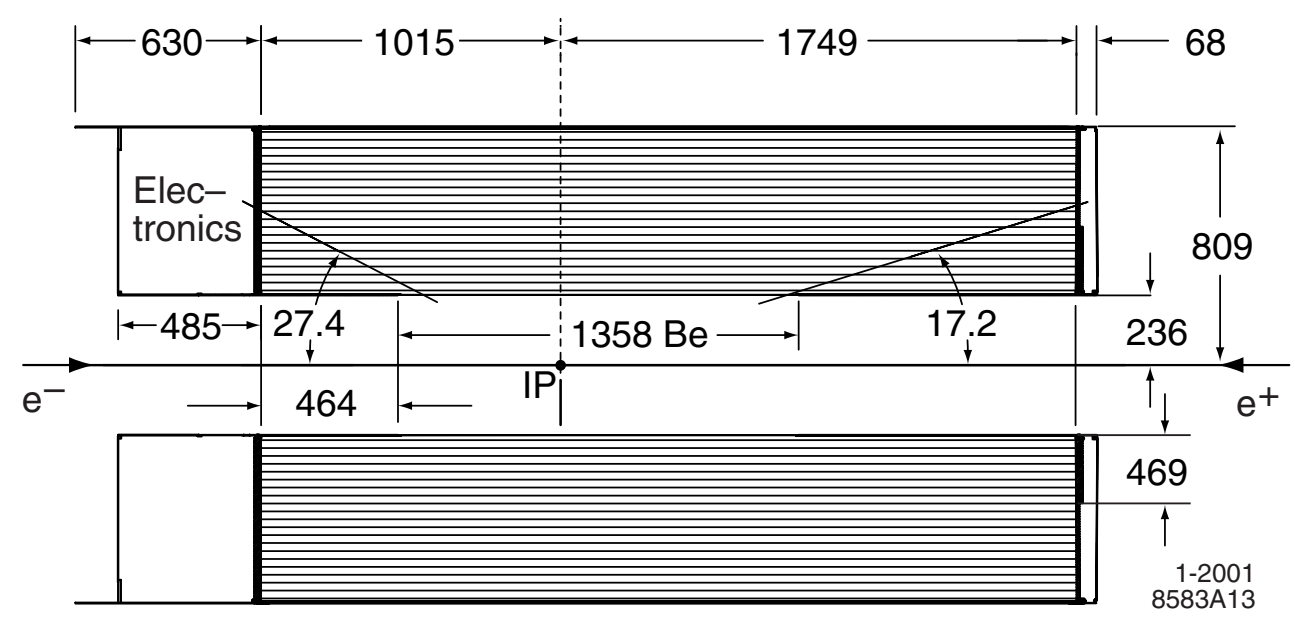

Figure 3.4: Schematic view of the DCH: longitudinal section.

To maximize the complementarity of devices, the DCH is designed with a thin inner cylindrical wall so that tracks may be matched with the SVT. This consideration applies to the outer wall as well so that performances of the outer subsystems are not compromised. The gas used in the chamber is an 80:20 mixture of helium with isobutane. This mixture yields an improvement in spatial resolution over past argonbased designs. The calibrated conversion of drift time to drift distance is determined from samples of electron and muon pairs. The effect of entrance angle has also been studied and corrected.

The DCH has performed reasonably well, and its performance is stable. In particular, it has met its design goal for intrinsic position $(140 \mu \mathrm{m})$ and $\mathrm{d} E / \mathrm{d} x$ resolution (7\%). Figure 3.5 shows $\mathrm{d} E / \mathrm{d} x$ measurements as a function of track momenta in the DCH. 


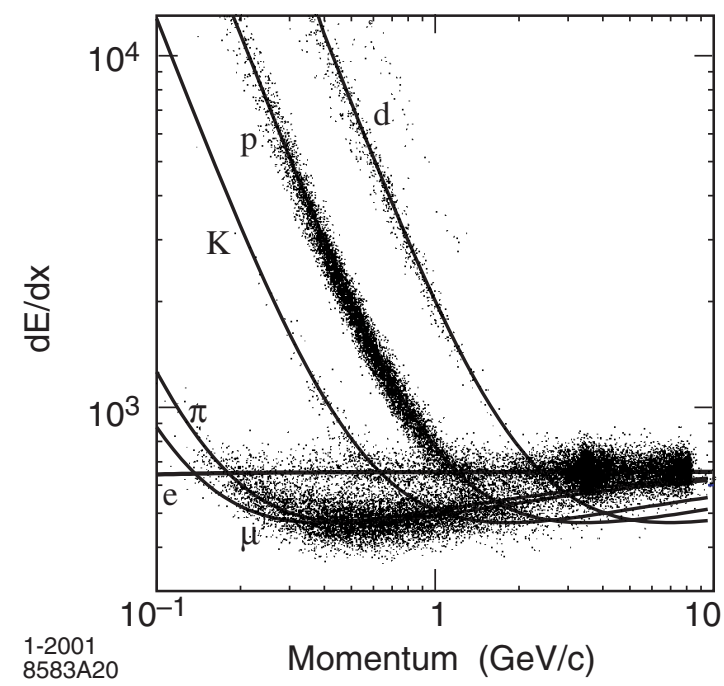

Figure 3.5: Measurement of $\mathrm{d} E / \mathrm{d} x$ in the DCH as a function of track momenta. The curves show the Bethe-Bloch predictions derived from selected control samples of particles of different masses.

\subsubsection{Tracking Performance}

The tracking system reconstructs charged tracks with high efficiency and sufficient resolution. The absolute DCH tracking efficiency is determined as the ratio of the number of reconstructed DCH tracks to the number of tracks detected in the SVT, with the requirement that the tracks fall within the acceptance region of the DCH. At the design voltage of $1960 \mathrm{~V}$, the efficiency averages $98 \% \pm 1 \%$ per track above $200 \mathrm{MeV} / \mathrm{c}$ and polar angle $\theta>500 \mathrm{mrad}$. In particular, tracking efficiency remains high for lowmomentum tracks, and SVT-only tracking extends the track-finding capability down to $\approx 50 \mathrm{MeV} / c$ (Figure 3.6).

Charged tracks are defined by five parameters, $\left(d_{0}, \phi_{0}, \omega, z_{0}, \tan \lambda\right)$, and their co- 
variance matrix. The parameters $\left(d_{0}, z_{0}\right)$ have already been defined in Section 3.1.1. The parameter $\phi_{0}$ is the azimuthal angle of the track, $\lambda$ is the dip angle relative to the transverse plane, and $\omega=1 / p_{t}$ is the track curvature. The resolutions of these parameters can be measured using cosmic-ray tracks that pass through the detector. The cosmic-ray detector hits are fit as two separate tracks, one in each half of the detector. The differences in track parameters for the two reconstructed tracks are shown in Figure 3.7 for tracks with transverse momenta above $3 \mathrm{GeV} / c$. Based on the full width at half maximum of these distributions, the resolutions for single tracks can be parameterized as

$$
\begin{aligned}
\sigma_{d_{0}} & =23 \mu \mathrm{m} \\
\sigma_{z_{0}} & =29 \mu \mathrm{m} \\
\sigma_{\phi_{0}} & =0.43 \mathrm{mrad} \\
\sigma_{\tan \lambda} & =0.53 \times 10^{-3} .
\end{aligned}
$$

The transverse-momentum resolution is determined to be

$$
\sigma_{p_{t}} / p_{t}=(0.13 \pm 0.01) \% \cdot p_{t}+(0.45 \pm 0.03) \%
$$

\subsection{Particle Identification}

Good separation between kaons and pions is crucial for the analysis undertaken in this work. The $B A B A R$ detector relies on ionization-loss $(\mathrm{d} E / \mathrm{d} x)$ measurements in the 


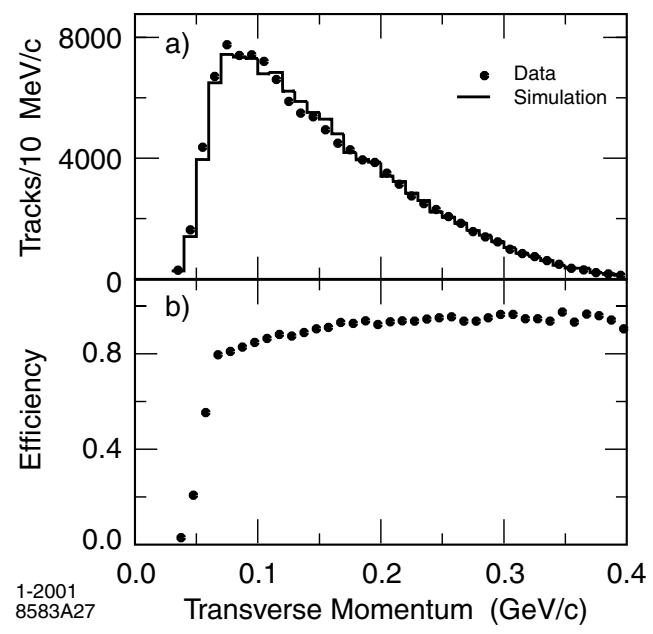

Figure 3.6: Monte Carlo studies of low momentum tracking in the SVT: a) comparison of data (contributions from combinatoric background and non- $B \bar{B}$ events have been subtracted) with simulation of the transverse momentum spectrum of pions from $D^{*+} \rightarrow D^{0} \pi^{+}$in $B \bar{B}$ events, and b) efficiency for soft pion detection derived from simulated events.

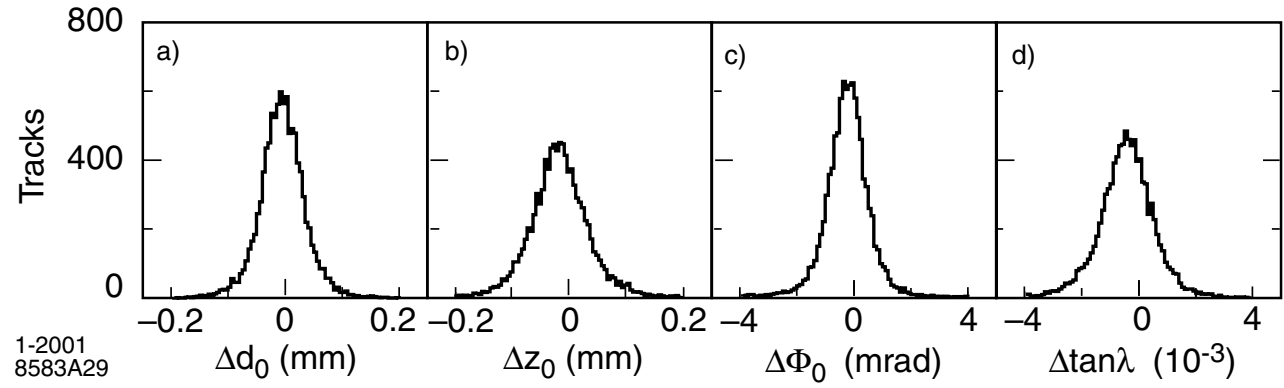

Figure 3.7: Measurements of the differences between the fitted track parameters of the two halves of cosmic ray muons, with transverse momenta above $3 \mathrm{GeV} / c$, a) $\Delta d_{0}$, b) $\Delta z_{0}$, c) $\Delta \phi_{0}$, and d) $\Delta \tan \lambda$. 
tracking system for particle identification (PID) of low momentum tracks. For tracks with transverse momentum above $700 \mathrm{MeV} / c, B A B A R$ has a dedicated PID system, the detector of internally-reflected Cherenkov light (DIRC). The DIRC is a uniquelydesigned ring-imaging Cherenkov detector, and some details are given below.

\subsection{1 $\mathrm{d} E / \mathrm{d} x$ in the Tracking System}

As noted above, the drift chamber can separate $K$ from $\pi$ with resolution of $7 \%$ up to $700 \mathrm{MeV} / c$ (Figure 3.5). In particular, for particles traveling in the extreme backward or forward direction, this is the only discriminating information available. Measurements of $\mathrm{d} E / \mathrm{d} x$ are also available from the SVT, but in this area it plays primarily a supporting role.

\subsubsection{Detector of Internally-Reflected Cherenkov Light}

Above $700 \mathrm{MeV} / c$, charged particles are identified in the dedicated PID system in $B A B A R$, known as the DIRC. This ring-imaging Cherenkov detector consists of 144 synthetic quartz bars oriented axially just beyond the drift chamber. Global design requirements mandate that the PID system must be thin and uniform to minimize resolution degradation of the calorimeter, located just outside it. Moreover, the resolution expectation of the DIRC is 4- $\sigma$ separation between $K$ and $\pi$.

A schematic of the important features of the DIRC is shown in Figure 3.8. The 


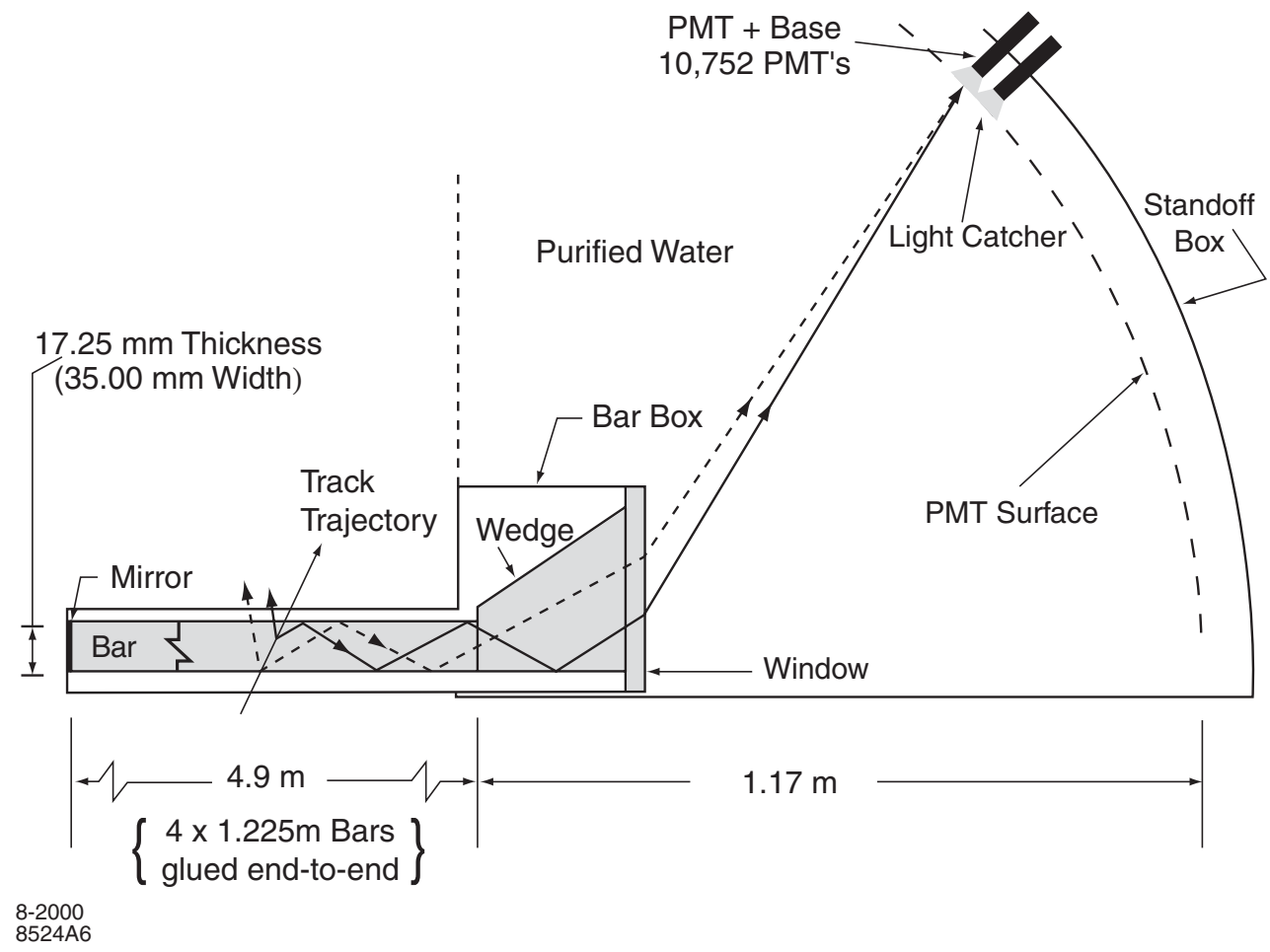

Figure 3.8: Schematic view of the DIRC fused silica radiator bar and imaging region.

device is designed on the principle that reflection from a flat surface preserves angular magnitudes. The quartz bars of the DIRC serve both as radiators and as light pipes for the portion of the light trapped by total internal reflection. The material chosen for these bars has many important qualities, such as resistance to ionizing radiation, long attenuation length, large index of refraction, and an excellent optical finish on the bars themselves. These radiators are arranged in a 12-sided barrel. The asymmetry of PEP-II produces particles in a preferentially-forward direction; therefore, the DIRC photon detector is placed at the backward end where it does not compete for space with front-end detecting components. Mirrors at the front end allow for this one-sided 


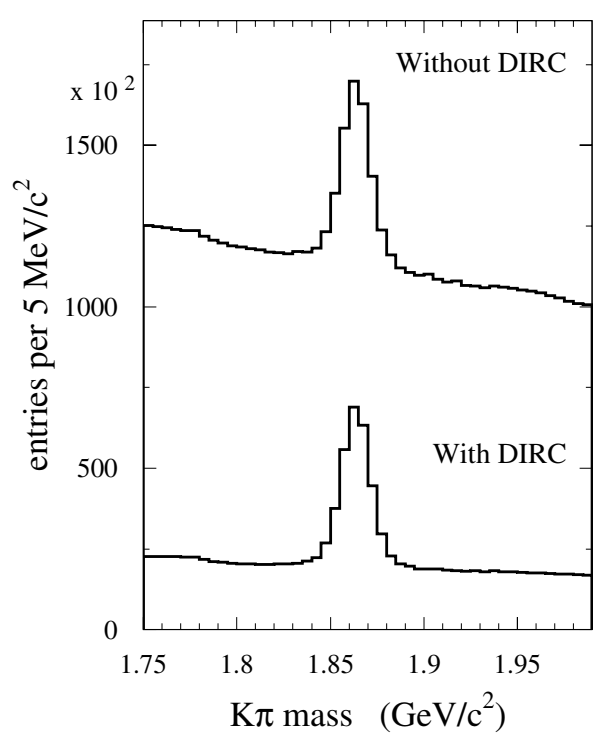

Figure 3.9: $D^{0} \rightarrow K \pi$ invariant mass distribution with and without the use of the DIRC for $K$ identification.

instrumentation.

The Cherenkov photons produced in the crystals are imaged by 11,000 conventional photomultiplier tubes. These are in a standoff box that contains about 6 kiloliters of purified water. Water is an inexpensive material that reasonably minimizes total internal reflection at the crystal interface. The water must be deionized and extremely pure to maintain good transparency for wavelengths down to $300 \mathrm{~nm}$.

The DIRC also has a nitrogen gas system, using liquid nitrogen boil-off both to prevent condensation on the radiators and to detect water leaks.

This clever and innovative PID device has proven to be robust and stable, and it is well-matched to the hadronic-PID requirements of BABAR. One can appreciate the 


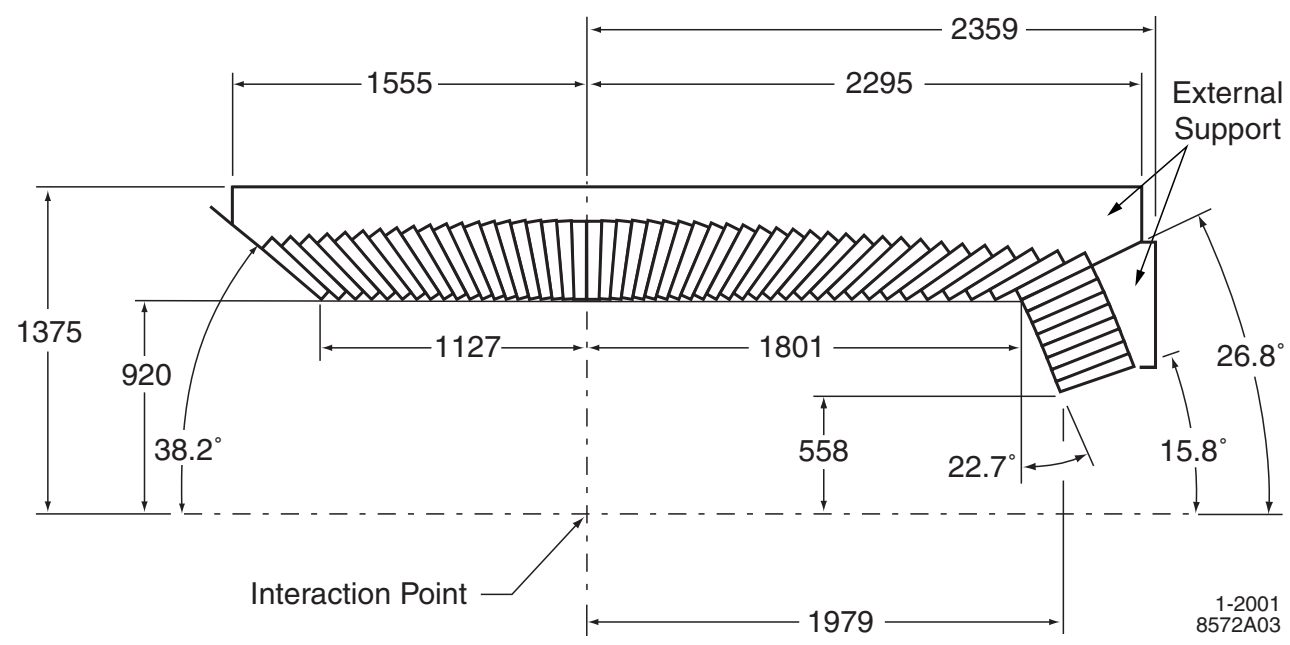

Figure 3.10: Schematic view of the EMC: longitudinal section. The detector is axially symmetric around the $z$-axis. All dimensions are given in millimeters

value of this system to physics analyses by considering Figure 3.9, which shows the $D^{0} \rightarrow K \pi$ invariant mass distribution with and without $K$-identification information from the DIRC. For what concerns this analysis, the $K$ and $\pi$ misidentification rates are shown in Appendix A, Section A.3.

\subsection{Reconstruction of $\pi^{0}$}

\section{in the Electromagnetic Calorimeter}

In addition to charged-particle tracks, this analysis is concerned with the reconstruction of $\pi^{0} \rightarrow \gamma \gamma$. Neutral particles are reconstructed in BABAR by the electromagnetic calorimeter (EMC) that is located just outside the DIRC.

For reconstruction of $\pi^{0} \mathrm{~s}$ and other neutral particles that are not detected by the 
tracking system, the EMC is designed to efficiently measure electromagnetic showers and to resolve energy and angles over the energy range from $20 \mathrm{MeV}$ to $9 \mathrm{GeV}$.

The physics goals of the BABAR experiment demand energy resolution of the order of $1-2 \%$. This is the dominant contribution to the $\pi^{0}$-mass resolution at energies below $2 \mathrm{GeV}$. Above this energy, the angular resolution is dominant; it is therefore required to be of the order of a few milliradians.

The EMC is a hermetic, total-absorption calorimeter, shown schematically in Figure 3.10. It consists of a fine array of thallium-doped cesium iodide (CsI(Tl)) crystals that are read out with silicon photodiodes. The crystals are arranged in 56 rings, and they are laid out to accommodate the asymmetry of the PEP-II facility. The EMC crystals are calibrated at the low end of the energy spectrum with a radioactive source, and at the high end with Bhabha events. The measured energy resolution is $\sigma_{E} / E=1.9 \pm 0.07 \%$ at $7.5 \mathrm{GeV}$. Figure 3.11 shows the energy resolution extracted from a variety of processes as a function of energy. The measured angular resolution is based on an analysis of $\pi^{0}$ and $\eta$ decays to two photons of approximately the same energy, and it is shown in Figure 3.12. The $\gamma \gamma$ invariant mass spectrum near the $\pi^{0}$ mass is shown in Figure 4.3 for this analysis. 


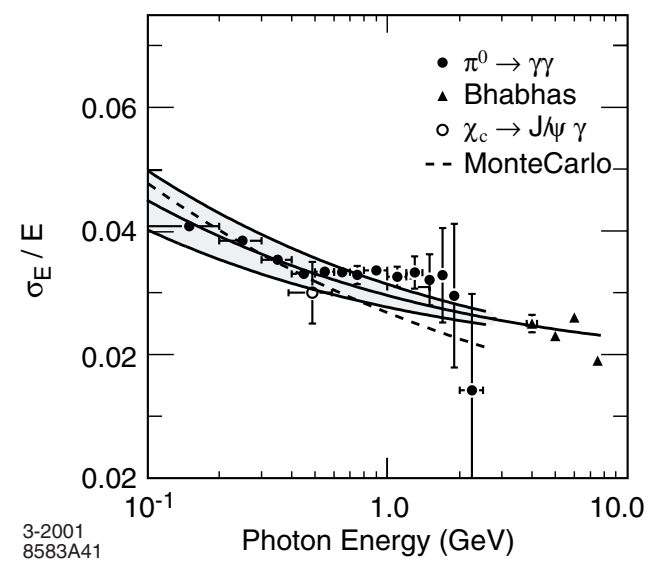

Figure 3.11: The energy resolution of the EMC measured for photons and electrons from various processes. The solid curve is a fit of an empirical resolution formula and the shaded area denotes the rms error of the fit.

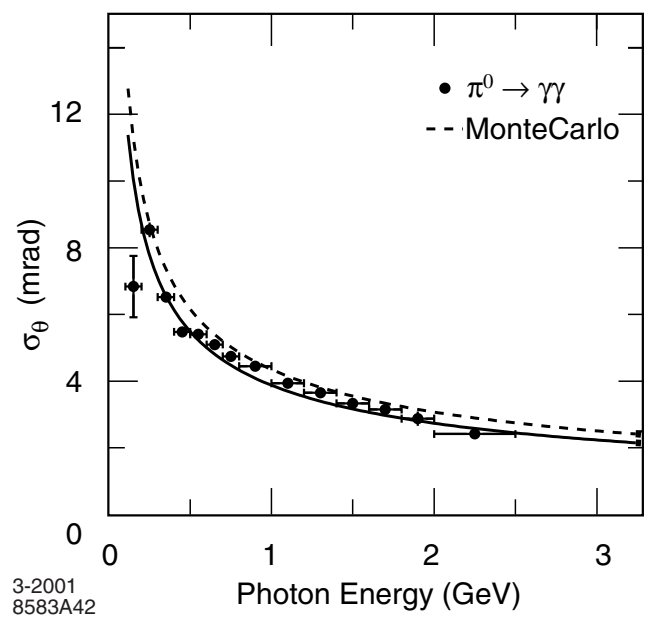

Figure 3.12: The angular resolution of the EMC for photons from $\pi^{0}$ decays. The solid curve is a fit of an empirical resolution formula. 


\section{Chapter 4}

\section{Event Selection}

\subsection{Overview of the Selection Process}

The size of the data set used for this analysis is $230.4 \mathrm{fb}^{-1}$, collected during 20002004 (Runs 1-4). The data set includes $e^{+} e^{-}$collisions at and slightly away from the $\Upsilon(4 S)$ resonance. The data set was always analyzed either in its entirety or randomlydivided subsets. Specific studies of systematic variation of the data, for example studies of distributions as a function of run number or detector conditions, were not performed for this analysis, although all data passed global quality measures established by the BABAR Collaboration. Monte Carlo events were generated using detector conditions sampled from characteristic periods representing the entire data set, and these simulated events were processed with the same reconstruction and analysis software 
as the recorded data.

The selection of candidates for this analysis proceeds in two steps. The first step is referred to as skimming, and the second step is referred to as reskimming. The reskimming step is done separately from the skimming step so that systematic studies of the selection criteria in the reskimming step can be performed. The skimming step is applied to the entire recorded and simulated data sets, and it consists of a few criteria that dramatically reduce the number of events under consideration. Because the data sets are so large, skimming is performed in a production environment, and the software applications are centrally organized and run by the BABAR computing group. The reskimming step is applied to the skimmed events, and it consists of more detailed selection criteria. In contrast to skimming, reskimming is managed by the analyst.

Most of the selection criteria used in this analysis have been studied in detail in related analyses performed previously by $B A B A R$, and as standard techniques for this sector of measurement, they are not scrutinized in detail for this work other than for applicability. The exceptions are the criteria for selecting $\pi^{0}$ candidates, which were studied in some detail. The $\pi^{0}$ selection criteria were determined by judiciously considering the distributions in question to eliminate high-background regions. 


\subsection{Skim Selection}

The skimming application SkimMiniApp was run on all Run 1-4 recorded data and simulated Monte Carlo events using the BABAR software releases 16.0.2e and 16.0.2f, producing the DmixDOToKPiPiO skim used for this analysis. Definitions of the variables used in the skim selection may be found in Table 4.1. The skim selection criteria are as follows:

- $\gamma$ candidates are taken from the GoodPhotonLoose list (see Appendix A)

- $0.01<$ EMC Lat $<0.8$ for each $\gamma$ candidate (see Figure 4.1)

- $100 \mathrm{MeV}<E_{\gamma}$ for each $\gamma$ candidate (see Figure 4.2)

- $\pi^{0}$ candidates are taken from the pi OVeryLoose list (see Appendix A), requiring the $\gamma$ criteria above and fitting $m_{\gamma \gamma}$ to the nominal $\pi^{0}$ mass.

- $0.01<\pi^{0}$ mass-fit $p\left(\chi^{2}\right)$ (see Figure 4.4)

- $K^{\mp}$ candidates are taken from the KLHTight list (see Appendix A)

- $\pi^{ \pm}$candidates (from the $D^{0}$ ) are taken from the piLHTight list (see Appendix A)

- The $K^{\mp}$ and $\pi^{ \pm}$tracks are fit to a common vertex using the FastVtx vertex fitter 
- $1.70<m_{K \pi \pi^{0}}<2.00 \mathrm{GeV} / c^{2}$

- $2.2 \mathrm{GeV} / c<p^{*}$ (see Figure 4.5)

- $0.005<D^{0}$ vertex-fit $p\left(\chi^{2}\right)$ (see Figure 4.6)

- The $\pi_{s}^{ \pm}$candidates (from the $D^{*+}$ ) are taken from the GoodTracksVeryLoose list (see Appendix A)

- The $D^{0}$ and $\pi_{s}^{ \pm}$are fit to a common vertex at the measured beamspot using the FastVtx vertex fitter

- $0.139<\Delta m<0.160 \mathrm{GeV} / c^{2}$

As one can see in Figures 4.1-4.3, the simulation of neutrals in the calorimeter does not match the data very well. One of the dominant reasons for this is that the $\gamma$ energy scale was calibrated differently in the simulation compared to the recorded data, and this mistake was corrected after the data was processed for this analysis. This analysis is relatively insensitive to these Monte Carlo inaccuracies because the two final states being compared are very similar. The only difference between the decay modes is in the population of the Dalitz plot. It is possible that the number of $\pi^{0} \mathrm{~s}$ selected in the two samples will have a different dependence on the $\pi^{0}$ momentum, but this question is addressed by determining a Dalitz-plot efficiency (Chapter 5).

The signal efficiency of this skim is estimated using $c \bar{c}$-continuum Monte Carlo and is listed in Table 4.2. This efficiency calculation is affected by the simulation 
Table 4.1: Definitions of skim variables
EMC Lat The lateral moment of the EMC cluster, defined as the ratio of $a$ to $b$ where
sum of energies of all but the two most energetic crystals
$a=$ in the cluster, weighted by the square of the distance to the cluster center
$b=\begin{aligned} & \text { sum of all cluster crystal energies weighted by } r^{2} \\ & r \text { is the length scale of the crystal }(5 \mathrm{~cm})\end{aligned}$
$E_{\gamma} \quad$ The lab energy of a $\gamma$ candidate
$\pi^{0} p\left(\chi^{2}\right) \quad$ The mass-fit $\chi^{2}$ probability from fitting $m_{\gamma \gamma}$ to the nominal $\pi^{0}$ mass
$m_{K \pi \pi^{0}} \quad$ The invariant mass of the $D^{0} \rightarrow K \pi \pi^{0}$ candidate
$p^{*} \quad$ The center-of-mass momentum of the $D^{0}$ candidate
$D^{0} p\left(\chi^{2}\right) \quad$ The vertex-fit $\chi^{2}$ probability from fitting the $K$ and $\pi$ tracks to a common vertex
$\Delta m \quad m_{K \pi \pi^{0} \pi_{s}}-m_{K \pi \pi^{0}}$, the invariant mass difference between the $D^{*+}$ candidate and the $D^{0}$ candidate

inaccuracies mentioned above, as well as a known inaccuracy in the simulated $p^{*}$ distribution; these uncertainties are not estimated or tabulated because the overall efficiency is irrelevant for the results of this analysis. The efficiency numbers are shown only to help understand the data sample in broad terms. The most significant cuts made in the skim for reducing background are the $p^{*}$ requirement and the particle identification requirements (the lists used for selecting $K^{\mp}$ and $\pi^{ \pm}$). 
Table 4.2: Estimated signal efficiency for skimming. The estimated error shown is statistical only.

\section{Monte Carlo sample}

generic $c \bar{c}$ total events

13650000

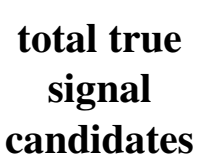

591700 reconstructed

true
signal
candidates

75661 signal

efficiency

$12.79 \% \pm 0.05 \%$

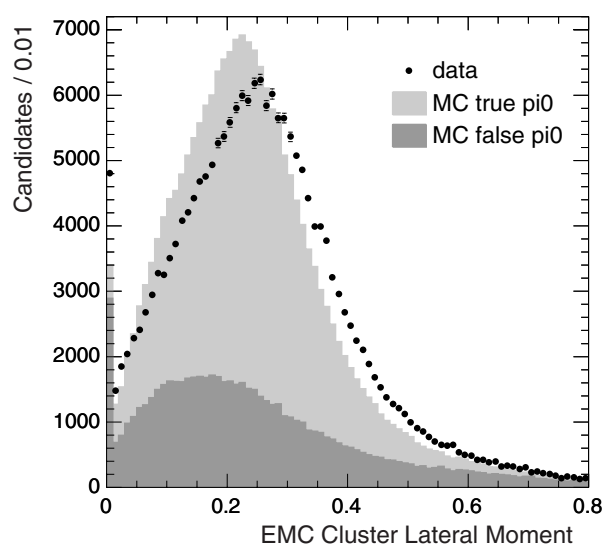

Figure 4.1: EMC cluster lateral moment distribution for approximately $5 \mathrm{fb}^{-1}$ of data and an equivalent number of weighted Monte Carlo events. All skim selection cuts except those on the distribution above have been applied. Monte Carlo events are shown as truth-matched distributions, in the case that the $\gamma$ candidate originated from a true $\pi^{0}$, and in the case that it did not. A relatively large number of background events have a value of 0.0 and are removed by requiring EMC Lat $>0.01$. 


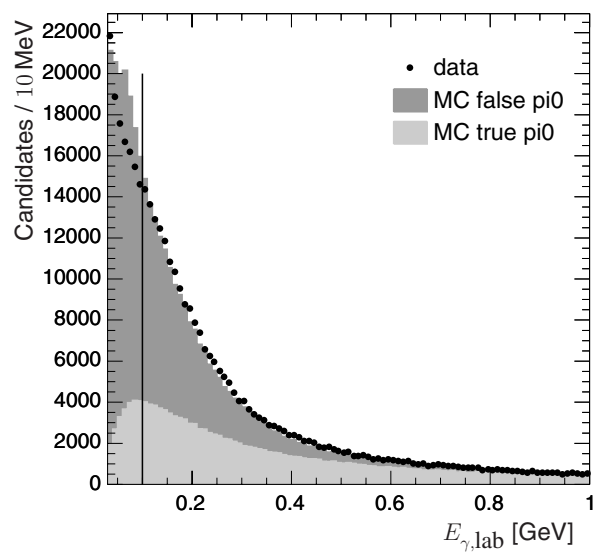

Figure 4.2: $\gamma$ energy distribution in the laboratory frame for approximately $5 \mathrm{fb}^{-1}$ of data and an equivalent number of weighted Monte Carlo events. The vertical line shows the skim cut on this distribution. All skim selection cuts except those on the distribution above have been applied. Monte Carlo events are shown as truth-matched distributions, in the case that the $\gamma$ candidate originated from a true $\pi^{0}$, and in the case that it did not.

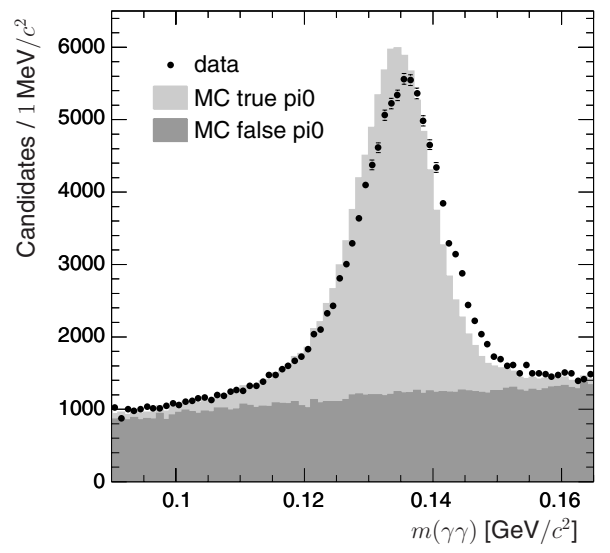

Figure 4.3: $\pi^{0}$ mass distribution for approximately $5 \mathrm{fb}^{-1}$ of data and an equivalent number of weighted Monte Carlo events. All skim selection cuts except those on the $\pi^{0}$ mass fit have been applied. Monte Carlo events are shown as truth-matched distributions, in the case that the $\pi^{0}$ candidate is a true $\pi^{0}$, and in the case that it is not. 

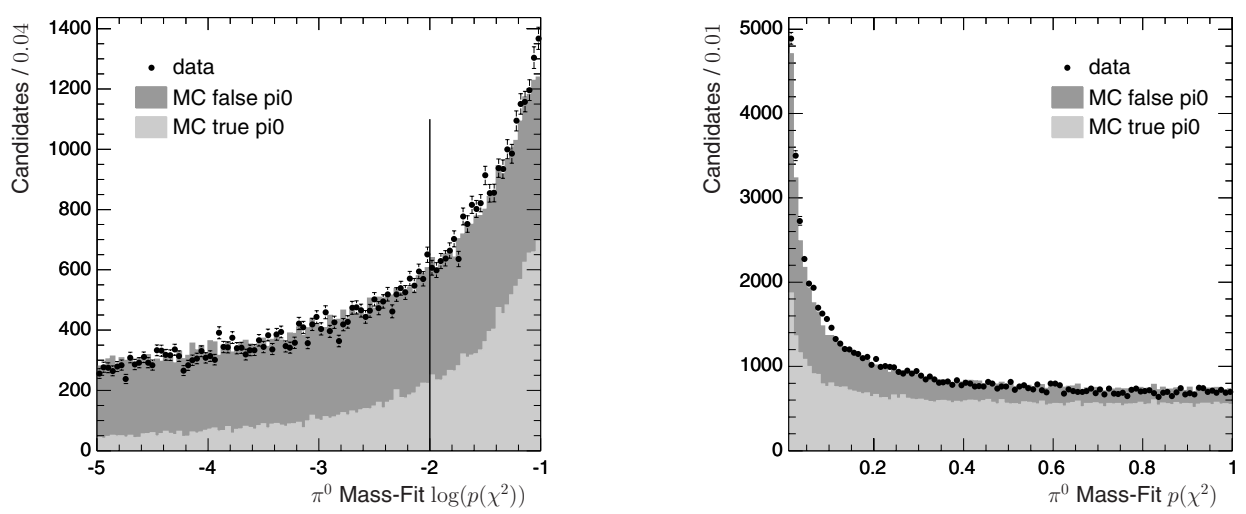

Figure 4.4: $\pi^{0}$ mass-fit $\chi^{2}$ probability distribution for approximately $5 \mathrm{fb}^{-1}$ of data and an equivalent number of weighted Monte Carlo events. The distribution is shown on both a log scale (left) and a linear scale (right). The vertical line on the left plot shows the skim cut on this distribution. All skim selection cuts except those on the distribution above have been applied. Monte Carlo events are shown as truth-matched distributions, in the case that the $\pi^{0}$ candidate is a true $\pi^{0}$, and in the case that it is not. 


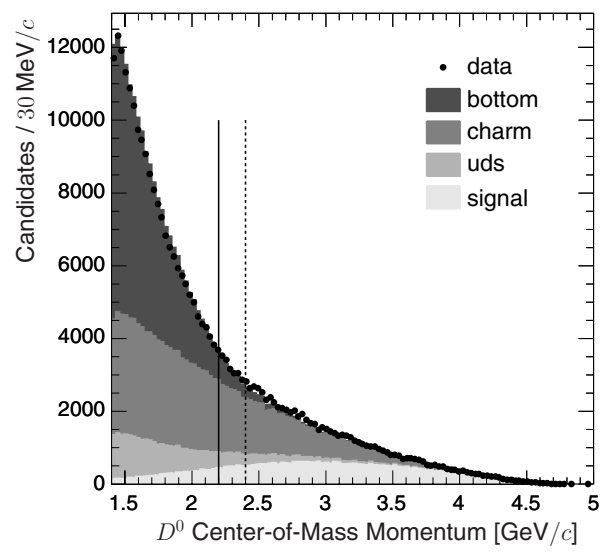

Figure 4.5: $D^{0}$ center-of-mass momentum distribution for approximately $5 \mathrm{fb}^{-1}$ of data and an equivalent number of weighted Monte Carlo events. The solid vertical line shows the cut made in the skim; the dotted vertical line shows the cut made in the reskim. All skim selection cuts except those on the distribution above have been applied. Monte Carlo events are shown as truth-matched distributions. The "bottom" category contains candidates, possibly with charm content, originating in $B$ decays. The "charm" category contains candidates originating from $c \bar{c}$ fragmentation that are not signal. The "uds" category contains candidates from light-quark fragmentation. The "signal" category contains $D^{*+} \rightarrow D^{0} \pi^{+}, D^{0} \rightarrow K^{\mp} \pi^{ \pm} \pi^{0}$ (+ C.C.) decays. 

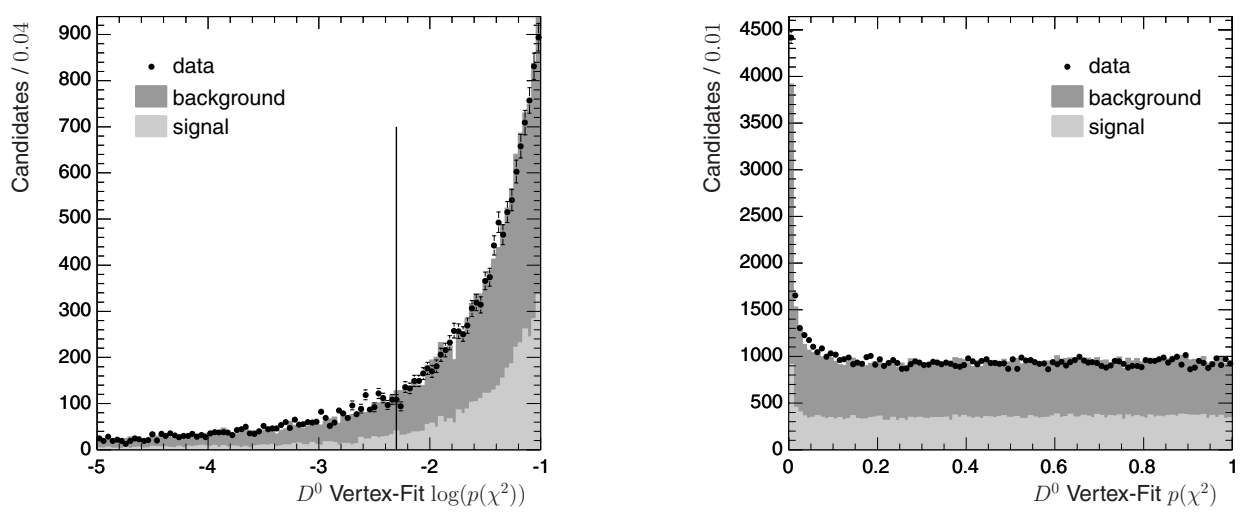

Figure 4.6: $D^{0}$ vertex-fit $\chi^{2}$ probability distribution for approximately $5 \mathrm{fb}^{-1}$ of data and an equivalent number of weighted Monte Carlo events. The distribution is shown on both a log scale (left) and a linear scale (right). The vertical line on the left plot shows the skim cut on this distribution. All skim selection cuts except those on the distribution above have been applied. Monte Carlo events are shown as truth-matched distributions, in the case that the $D^{0}$ candidate is a true signal $D^{0}$, and in the case that it is not. 


\subsection{Reskim Selection}

The reskimming application DmixKpipiOUserApp was run on all of the skimmed data and Monte Carlo in the DmixD0ToKPiPiO collections described above using the BABAR software release 16.0.3-physics-1 (analysis-24). Definitions of the variables used in the reskim selection can be found in Table 4.3. The reskim selection criteria are as follows:

- Candidates are taken from the list produced in the skim, described above

- $350 \mathrm{MeV} / c<p_{\pi^{0}}$ (see Figure 4.7)

- The $K^{\mp}$ candidate must be included in the KLHVeryTight list (see Appendix A)

- The $\pi^{ \pm}$candidate (from the $D^{0}$ ) must be included in the piLHVeryTight list (see Appendix A)

- The $\pi_{s}^{ \pm}$candidate (from the $D^{*+}$ ) is selected only if it has hits in the drift chamber

- The entire decay chain is fit using the TreeFitter fitter

- $0.01<p\left(\chi^{2}\right)$ (see Figure 4.8)

- $1.70<m_{K \pi \pi^{0}}<2.00 \mathrm{GeV} / c^{2}$ (value is obtained from the full decay chain fit) 
Table 4.3: Definitions of reskim variables

$$
\begin{aligned}
& p_{\pi^{0}} \quad \text { The } \pi^{0} \text { lab-momentum magnitude } \\
& p\left(\chi^{2}\right) \quad \text { The } \chi^{2} \text { probability of the fit to the entire decay chain: } D^{*+} \rightarrow D^{0} \pi^{+} \\
& \text {(common vertex constrained to be at the measured beamspot), } D^{0} \rightarrow \\
& K \pi \pi^{0} \text { (common vertex for the tracks), } \pi^{0} \rightarrow \gamma \gamma\left(\pi^{0}\right. \text { mass con- } \\
& \text { strained to the PDG value). }
\end{aligned}
$$

- $2.4 \mathrm{GeV} / c<p^{*}$ (see Figure 4.5)

- $0.139<\Delta m<0.155 \mathrm{GeV} / c^{2}$ (value is obtained from the full decay chain fit)

After the above selection criteria have been applied, often there remain two or more candidates in an event. Those candidates that have a track or cluster in common are referred to as overlapping candidates. The following checks are applied to overlapping candidates in an event to select a single candidate.

1. If two $\pi^{0}$ candidates share a $\gamma$, the $\pi^{0}$ in the decay with the higher $p\left(\chi^{2}\right)$ value is selected. Approximately 5\% of events exhibit this.

2. If two $D^{0}$ candidates share a $\pi^{0}$, the $D^{0}$ in the decay with the higher $p\left(\chi^{2}\right)$ value is selected. Approximately $2 \%$ of events exhibit this.

3. Consider two $D^{*+}$ candidates reconstructed as follows:

$$
\begin{aligned}
& D^{*+} \rightarrow D^{0} \pi_{1}^{+}, \quad D^{0} \rightarrow K^{-} \pi_{2}^{+} \pi^{0} \\
& D^{*+} \rightarrow D^{0} \pi_{2}^{+}, \quad D^{0} \rightarrow K^{-} \pi_{1}^{+} \pi^{0}
\end{aligned}
$$


It is found that the ambiguity can be resolved correctly in $90 \%$ of these cases by choosing the candidate that has mass values $m_{K \pi \pi^{0}}$ and $\Delta m$ closer to the expected signal values. This ambiguity occurs in cases where the $D^{0}$ decays through the channel $D^{0} \rightarrow K^{-} \rho^{+}$. If the $\rho^{+}$is emitted in the direction of the $D^{0}$ momentum, its consequent longitudinal decay will often produce a $\pi^{+}$that is traveling with almost the same momentum magnitude and direction as the $\pi^{+}$ from the $D^{*+}$ decay. Approximately $6 \%$ of events exhibit this.

4. If two $D^{*+}$ candidates share either a $D^{0}$ or a $\pi_{s}^{+}$candidate, the $D^{*+}$ with the higher $p\left(\chi^{2}\right)$ value is selected. Approximately $0.6 \%$ of events exhibit this.

5. If two $D^{0}$ candidates share one track and one $\pi^{0}$, the $D^{0}$ with the higher $p\left(\chi^{2}\right)$ value is selected. Fewer than $0.1 \%$ of events exhibit this.

6. If two $D^{0}$ candidates share both tracks, the $D^{0}$ with the higher $p\left(\chi^{2}\right)$ value is selected. Fewer than $0.05 \%$ of events exhibit this.

Approximately $0.15 \%$ of events have two reconstructed $D^{*+}$ candidates with no shared tracks or clusters. In these cases, both of the candidates are kept. This fraction agrees generally with expectations, based on charm fragmentation and the branching fractions involved.

An unusual selection criterion was considered for this analysis:

- If a $D^{0}$ can be associated with either a $\pi^{+}$(right-sign) or a $\pi^{-}$(wrong-sign), 
the right-sign combination is selected, as its prior probability is two orders of magnitude higher. Approximately $7 \%$ of wrong-sign events exhibit this.

This selection, though not used, was studied to determine its potential bias on the doubly Cabibbo-suppressed (DCS) $D^{0} \rightarrow K^{+} \pi^{-} \pi^{0}$ branching ratio measurement. It was found that $0.5 \%$ of DCS signal events are eliminated, but $10 \%$ of background events due to Cabibbo-favored $D^{0} \rightarrow K^{-} \pi^{+} \pi^{0}$ decays with an uncorrelated $\pi_{\mathrm{s}}^{\mp}$ are also removed. If the mixing portion of this analysis is repeated in the future, it is recommended that this selection be included. Since this work includes explicitly a branching ratio measurement, this technique was omitted for clarity.

As with the skim, the reskim efficiency is estimated using $c \bar{c}$-continuum Monte Carlo and is listed in Table 4.4. Combining the efficiencies for the skim and the reskim, the estimated overall signal efficiency for the event selection is

$$
\epsilon_{\text {skim }} \epsilon_{\text {reskim }}=6.26 \% \pm 0.03 \% \text {. }
$$


Table 4.4: Estimated signal efficiency for reskimming. The estimated error shown is statistical only.

$\begin{array}{ccccc}\begin{array}{c}\text { Monte Carlo } \\ \text { sample }\end{array} & \text { total events } & \begin{array}{c}\text { total true } \\ \text { signal } \\ \text { candidates }\end{array} & \begin{array}{c}\text { reconstructed } \\ \text { true } \\ \text { signal } \\ \text { candidates }\end{array} & \begin{array}{c}\text { signal } \\ \text { efficiency }\end{array} \\ \begin{array}{c}\text { generic } c \bar{c} \\ \text { (skimmed) }\end{array} & 6451799 & 3030691 & 1483826 & 48.96 \% \pm 0.04 \%\end{array}$

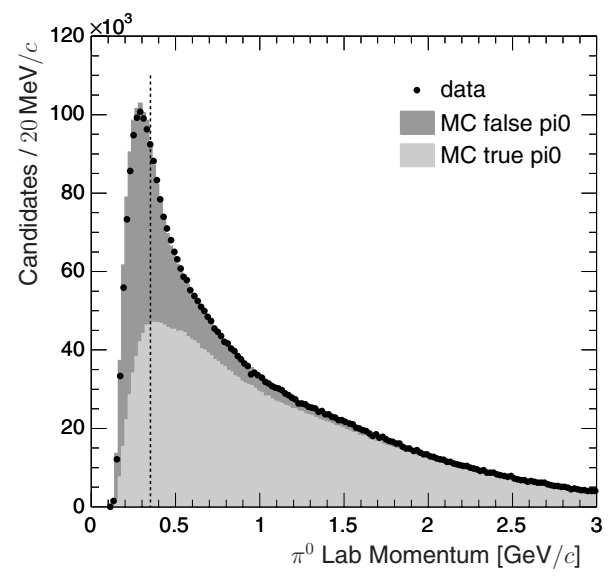

Figure 4.7: $\pi^{0}$ lab momentum distribution for all of the skimmed data and Monte Carlo, where the Monte Carlo distributions have been rescaled to match the number of events in data. The dotted vertical line shows the reskim cut on this distribution. All skim selection cuts, and reskim selection cuts on $p^{*}, p\left(\chi^{2}\right), m_{K \pi \pi^{0}}$, and $\Delta m$, have been applied. Monte Carlo events are shown as truth-matched distributions, in the case that the $\pi^{0}$ candidate is a true $\pi^{0}$, and in the case that it is not. 

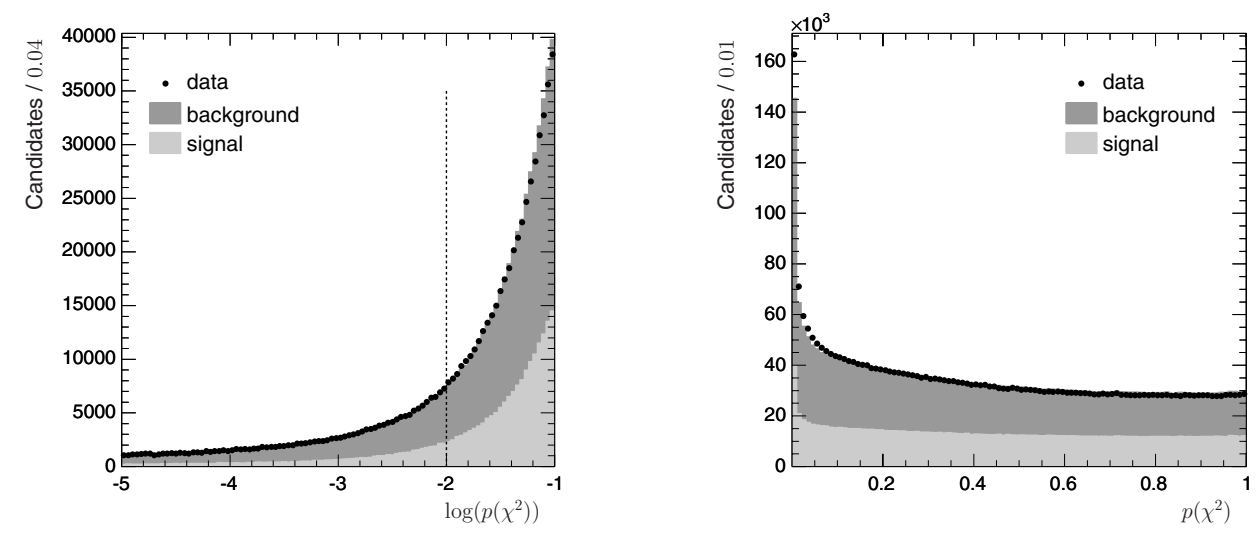

Figure 4.8: $\chi^{2}$ probability distribution for the fit of the entire decay chain for all of the skimmed data and Monte Carlo, where the Monte Carlo distributions have been rescaled to match the number of events in data. The fit performed is: $D^{*+} \rightarrow$ $D^{0} \pi^{+}$(common vertex constrained to be at the measured beamspot), $D^{0} \rightarrow K \pi \pi^{0}$ (common vertex for the tracks), $\pi^{0} \rightarrow \gamma \gamma$ ( $\pi^{0}$ mass constrained to the PDG value). The distribution is shown on both a log scale (left) and a linear scale (right). The vertical dotted line on the left plot shows the reskim cut on this distribution. All skim selection cuts, and reskim selection cuts on $p^{*}, p\left(\chi^{2}\right), m_{K \pi \pi^{0}}$, and $\Delta m$, have been applied. Monte Carlo events are shown as truth-matched distributions, in the case that the $D^{0}$ candidate is a true signal $D^{0}$, and in the case that it is not. 


\section{Chapter 5}

\section{Dalitz-Plot Efficiency}

Reconstruction efficiencies for the doubly Cabibbo-suppressed (DCS) $D^{0} \rightarrow K^{+} \pi^{-} \pi^{0}$ decay and the Cabibbo-favored (CF) $D^{0} \rightarrow K^{-} \pi^{+} \pi^{0}$ decay are very similar, since in both cases the same basic decay tree is being reconstructed. The only significant difference between the CF and DCS decays is the manner in which they populate the Dalitz plot. In order to account for this difference when determining final values, a Dalitz-plot efficiency correction is applied to the data. This correction does not need to be correct on an absolute scale, since in all cases of interest, final values involve only ratios of DCS values to CF values.

The Dalitz-plot efficiency is evaluated separately for

$$
\begin{aligned}
& D^{*+} \rightarrow D^{0} \pi_{s}^{+}, D^{0} \rightarrow K^{-} \pi^{+} \pi^{0} \\
& D^{*-} \rightarrow \bar{D}^{0} \pi_{s}^{-}, \bar{D}^{0} \rightarrow K^{+} \pi^{-} \pi^{0}
\end{aligned}
$$


using $2.3 \times 10^{6}$ simulated $D^{*+}$ events and $2.3 \times 10^{6}$ simulated $D^{*-}$ events, where the $D^{*+}$ and $D^{*-}$ are allowed to decay isotropically. For each of the two decays above, two samples are collected: a generated sample, and a reconstructed sample.

The generated sample comprises all generated signal decays, identified using the truth information stored with the simulated event. For this sample, the momenta of the particles are generated values, not reconstructed values. Therefore, this sample has a sharp, well-defined Dalitz-plot boundary.

The reconstructed sample comprises reconstructed candidates that have passed all of the analysis selection criteria. Since the momenta of these candidates are the reconstructed values, the Dalitz-plot boundary is smeared by the detector resolution. In order to have a well-defined Dalitz-plot boundary, these candidates are fitted with a $D^{0}$-mass constraint after passing all of the normal selection criteria, and the momenta of the $K, \pi$, and $\pi^{0}$ after applying the mass constraint are used.

The Dalitz plot in $m^{2}\left(\pi \pi^{0}\right)$ and $m^{2}\left(K \pi^{0}\right)$ is binned with 50 divisions on $0.0<$ $m^{2}\left(\pi \pi^{0}\right)<2.0$ and 75 divisions on $0.0<m^{2}\left(K \pi^{0}\right)<3.2$ (where $m^{2}$ is in units of $\left.\left[\mathrm{GeV} / c^{2}\right]^{2}\right)$. For each decay, this results in approximately 1200 generated events per bin (Figure 5.1). Bins containing fewer than 1000 generated events are removed, so that the low-statistics bins on the boundary do not affect the fit.

The Dalitz-plot efficiency is obtained by dividing the binned reconstructed sample 
by the binned generated sample. For each efficiency bin, the bin error is given by

$$
\sigma_{\epsilon}=\frac{1}{N_{g}^{2}} \sqrt{N_{g} N_{r}\left(N_{g}-N_{r}\right)},
$$

where $N_{g}$ is the number of generated events and $N_{r}$ is the number of reconstructed events in that bin. A two-dimensional, third-order polynomial is fit to the binned efficiency by minimizing the $\chi^{2}$ value. The polynomial is parameterized as

$$
\begin{aligned}
\epsilon(x, y)= & a_{0}\left(1+a_{1} x+a_{2} y+a_{3} x^{2}+a_{4} y^{2}+a_{5} x y\right. \\
& \left.+a_{6} x^{3}+a_{7} y^{3}+a_{8} x y^{2}+a_{9} x^{2} y\right) .
\end{aligned}
$$

For the $D^{*+}$ decay, $\chi^{2}=2386$ for 1932 bins; for the $D^{*-}$ decay, $\chi^{2}=2429$ for 1928 bins. Both the raw binned efficiency and the fitted efficiency polynomial for the $D^{*+}$ decay are shown in Figure 5.2, and the projections of the efficiency are shown in Figure 5.3. Pull distributions for the fit are shown in Figure 5.4, and they confirm that the polynomial describes the efficiency well.

There are a couple of aspects of this method for determining efficiency that may cause concern. First, it is assumed that a simulated candidate will be reconstructed in the same efficiency bin in which it was generated. This is believed to be an insignificant effect because of the $D^{0}$-mass constraint applied to the candidates considered in this efficiency study. However, this assumption has not been explicitly verified. Second, to the extent that the treatment of $\pi^{0} \mathrm{~s}$ in simulated events and in recorded data is different, the binned efficiency may not accurately reflect the actual Dalitz- 


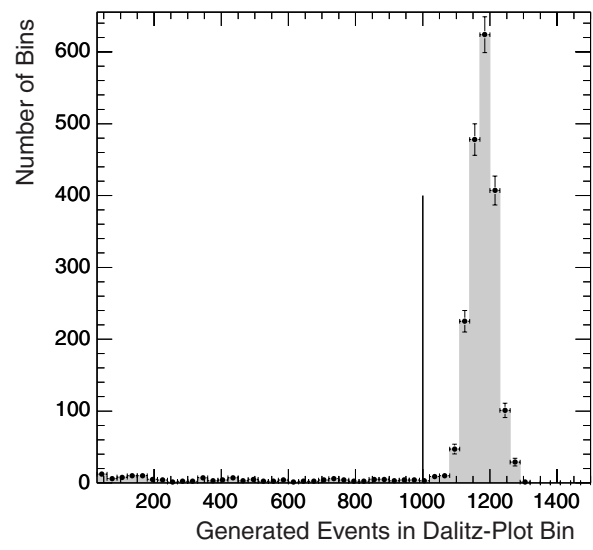

Figure 5.1: Number of generated $D^{*+} \rightarrow D^{0} \pi_{s}^{+}, D^{0} \rightarrow K^{-} \pi^{+} \pi^{0}$ events in Dalitz-plot bins, where the Dalitz plot has been binned with 50 divisions for $0.0<$ $m^{2}\left(\pi \pi^{0}\right)<2.0\left[\mathrm{GeV} / c^{2}\right]^{2}$ and 75 divisions for $0.0<m^{2}\left(K \pi^{0}\right)<3.2\left[\mathrm{GeV} / c^{2}\right]^{2}$. The vertical line indicates the cut made on this distribution.
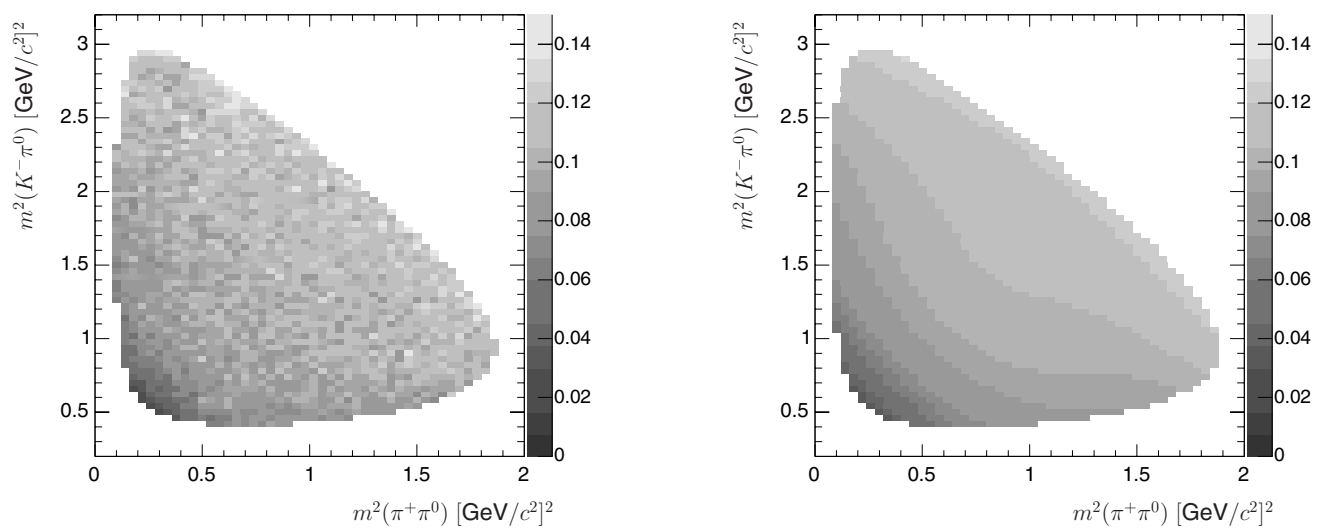

Figure 5.2: Efficiency of reconstructing $D^{*+} \rightarrow D^{0} \pi_{s}^{+}, D^{0} \rightarrow K^{-} \pi^{+} \pi^{0}$ over the Dalitz plot, binned (left) and parameterized as a two-dimensional, third-order polynomial (right). Corresponding histograms for the $D^{*-}$ decay are substantively the same. 

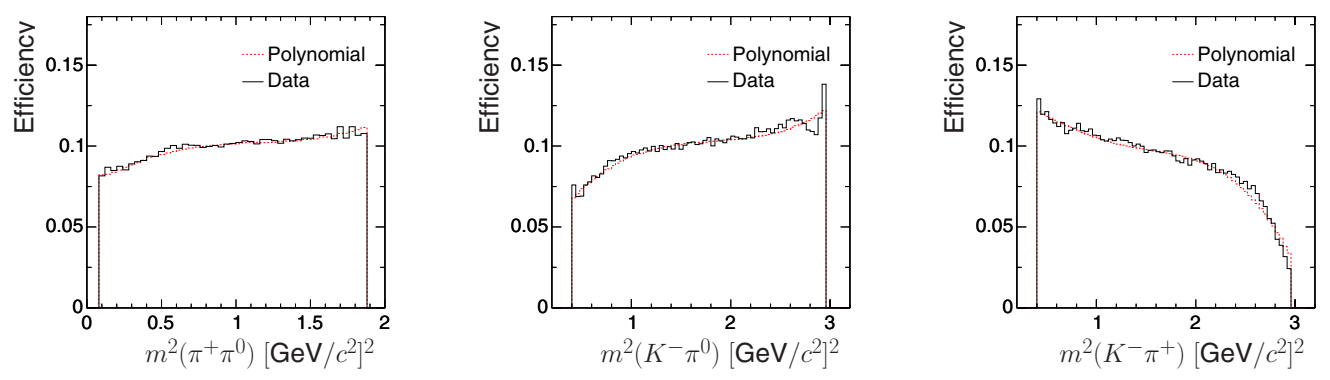

Figure 5.3: Average efficiency of reconstructing $D^{*+} \rightarrow D^{0} \pi_{s}^{+}, D^{0} \rightarrow K^{-} \pi^{+} \pi^{0}$, from the raw binned efficiency histogram and from the third-order polynomial, projected onto the $m^{2}\left(\pi^{+} \pi^{0}\right)$ (left), $m^{2}\left(K^{-} \pi^{0}\right)$ (center), and $m^{2}\left(K^{-} \pi^{+}\right)$(right) distributions. Corresponding histograms for the $D^{*-}$ decay are substantively the same.
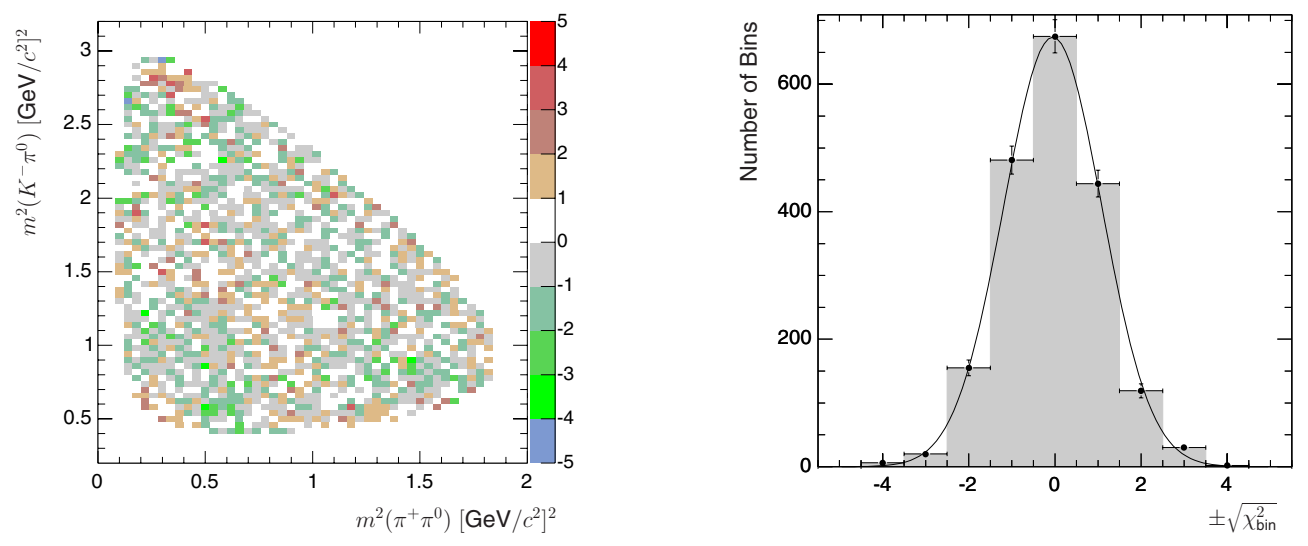

Figure 5.4: Pull $\left( \pm \sqrt{\chi^{2}}\right)$ distribution over the Dalitz region (left) and integrated over all bins (right) of the binned-efficiency fit for the $D^{*+} \rightarrow D^{0} \pi_{s}^{+}, D^{0} \rightarrow K^{-} \pi^{+} \pi^{0}$ decay. A Gaussian is fit to the pull distribution on the right, with a mean of $(-5.1 \pm$ 2.6) $\times 10^{-2}$ and $\sigma=1.14 \pm 0.02$. Corresponding histograms for the $D^{*-}$ decay are substantively the same. 
plot efficiency. The absolute $\pi^{0}$ reconstruction efficiency in BABAR is only known with an accuracy of (at best) $\pm 5 \%$. For the overall $D^{0} \rightarrow K^{+} \pi^{-} \pi^{0}$ branching ratio measurement, it is expected that this uncertainty will cancel to a substantial extent. This is because there is an approximate symmetry between the DCS and CF Dalitz plots with respect to $\pi^{0} \mathrm{~s}$. To see this, consider the simple case where the DCS Dalitz plot is the same as the CF Dalitz plot after exchanging $K$ with $\pi$. When applying an efficiency correction, both the variation in the efficiency and the dominant uncertainty are associated with the $\pi^{0}$; therefore, in this simple case, the systematic uncertainties in the efficiency correction will cancel when forming DCS-to-CF ratios. Indeed, an approximate symmetry applies to the efficiency correction (Figure 5.3) as well as the physical resonance structures observed in data (see Chapter 9). Furthermore, the efficiency correction applied to the branching ratio in Chapter 8 amounts to a change of a relative $3 \%$ in the final result, which demonstrates that the efficiency correction affects the DCS and CF decays similarly. Thus, it is asserted that the uncertainty due to $\pi^{0}$ reconstruction efficiency is effectively absorbed by the overly conservative systematic uncertainty in the efficiency correction for the branching ratio measurement. 


\section{Chapter 6}

\section{Maximum Likelihood Fit to $m_{K \pi \pi^{0}}$}

\section{and $\Delta m$ Distributions}

\subsection{Introduction}

The final separation of candidates into signal and background categories is accomplished by means of an unbinned maximum likelihood fit. In addition to allowing the shape parameters to vary during the minimization process, the numbers of candidates in the various signal and background categories are also allowed to vary. The probability of obtaining a particular number of candidates in a certain category obeys a Poisson distribution. The likelihood function is thus defined as

$$
\mathcal{L}\left(\vec{x}, \vec{p}, n_{1}, \ldots, n_{m}\right)=\frac{\exp \left(-\sum_{j=1}^{m} n_{j}\right)}{N !} \prod_{i=1}^{N}\left(\sum_{j=1}^{m} n_{j} \mathcal{P}_{j}(\vec{x}, \vec{p})\right),
$$


where $\vec{x}$ is the vector of observable dependent variables, $\vec{p}$ is the vector of shape parameters, $n_{1} \ldots n_{m}$ are the numbers of candidates in the $m$ signal and background categories, $N$ is the total number of candidates, and $\mathcal{P}_{j}(\vec{x}, \vec{p})$ are the probability density functions (PDFs) of the $m$ categories.

The two observables in the fit are

- $m_{K \pi \pi^{0}}$, the mass of the reconstructed $D^{0}$ candidate

- $\Delta m \equiv m_{K \pi \pi^{0} \pi_{s}}-m_{K \pi \pi^{0}}$, the mass difference between the $D^{*+}$ and $D^{0}$ candidates.

A two-dimensional fit is necessary because each distribution has a significant peaking background in the signal region. These backgrounds are described below. In order to reduce systematic uncertainties due to the shapes used in the fit, the same signal shape is used for both the Cabibbo-favored right-sign sample and the doubly Cabibbosuppressed or mixed wrong-sign sample, and the two samples are fit simultaneously. The ratio between the fitted numbers of signal candidates in the two samples is thus not biased by imperfect knowledge of the exact signal shape. One might argue that the signal shapes for right-sign and wrong-sign signal events may be slightly different because the Dalitz plots are populated differently, and hence the $\pi^{0}$ candidates in the two samples have slightly different momentum distributions, which may affect the signal shape. However, comparison of the fit projections in Figure 6.10 with those in Figure 6.11 demonstrates that the signal shapes are in fact the same. 


\subsection{Descriptions of Fit Categories and Probability Density Functions}

\subsubsection{Signal}

The signal category describes candidates that come from the decays

$$
\begin{aligned}
D^{*+} \rightarrow & D^{0} \pi_{s}^{+} \\
& \left.D^{0} \rightarrow \quad K^{\mp} \pi^{ \pm} \pi^{0} \quad \text { (+ C.C. }\right) .
\end{aligned}
$$

The distributions of both $m_{K \pi \pi^{0}}$ and $\Delta m$ have significant peaks for these candidates. The peak in the $m_{K \pi \pi^{0}}$ distribution is wider than the mass distribution obtained when reconstructing a $D^{0}$ candidate using only charged tracks, as in $D^{0} \rightarrow K^{-} \pi^{+}$. This broadened peak is due to the greater uncertainty in reconstructing the $\pi^{0}$ momentum. The $\Delta m$ peak is much narrower, as the uncertainty in the reconstruction of the $D^{0}$ candidate is absent to first order. However, as shown in Figure 6.1, there is some correlation between the $m_{K \pi \pi^{0}}$ and $\Delta m$ distributions. The width of the $\Delta m$ distribution depends on the distance from the $m_{K \pi \pi^{0}}$ distribution mean. The large number of events in the right-sign sample makes this correlation statistically relevant.

The two-dimensional PDF used to describe the signal category is illustrated in Figure 6.2 with projections onto the $m_{K \pi \pi^{0}}$ and $\Delta m$ axes. The shapes used as com- 


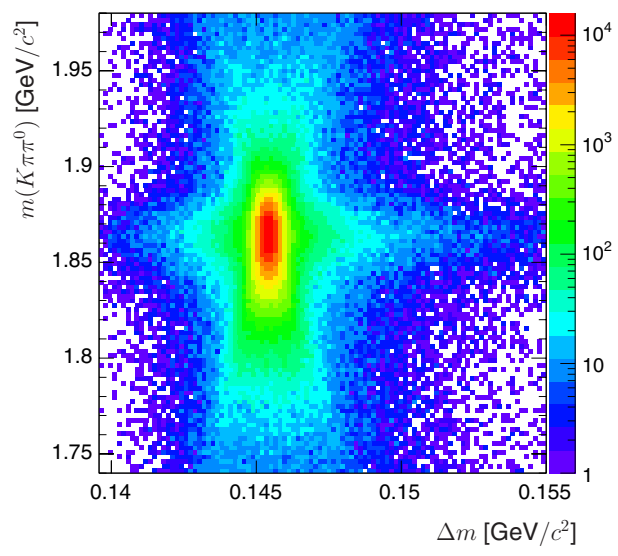

Figure 6.1: A two-dimensional histogram of $m_{K \pi \pi^{0}}$ versus $\Delta m$ for truth-matched right-sign signal candidates in Monte Carlo. The $\Delta m$ distribution width grows as a function of distance from the $m_{K \pi \pi^{0}}$ distribution mean.
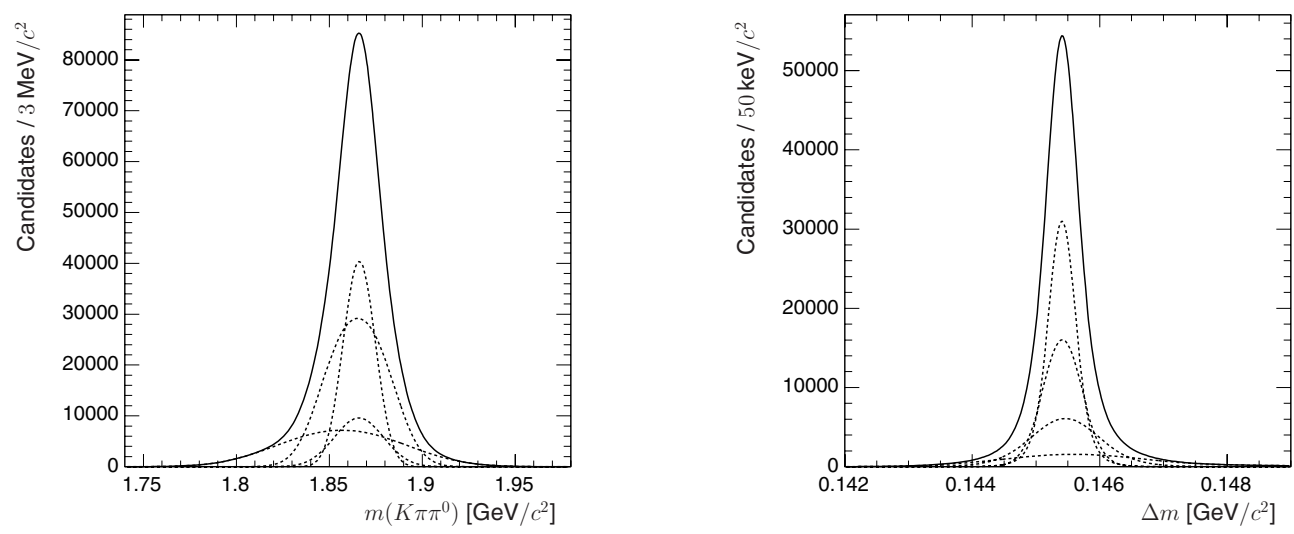

Figure 6.2: Graphs illustrating the components (dashed) of the signal PDF (solid) for the $m_{K \pi \pi^{0}}$ projection (left) and the $\Delta m$ projection (right). The dashed lines represent $s_{1}, s_{2}, s_{3}$, and $s_{4}$, described in the text. 
ponents of the PDF are

$$
\begin{gathered}
g(x ; \bar{x}, \sigma)=\exp \left(-\frac{(x-\bar{x})^{2}}{2 \sigma^{2}}\right) \\
c b_{s}(x ; \bar{x}, \sigma, \alpha)=\left\{\begin{array}{cc}
\exp \left(-\frac{(x-\bar{x})^{2}}{2 \sigma^{2}}\right) & \text { if } \frac{(x-\bar{x})}{\sigma}<\alpha \\
a\left(b+\frac{(x-\bar{x})}{\sigma}\right)^{-2} & \text { if } \frac{(x-\bar{x})}{\sigma} \geq \alpha
\end{array}\right. \\
\alpha>0, \quad a=(2 / \alpha)^{2} \exp \left(\alpha^{2} / 2\right), \quad b=(2 / \alpha)-\alpha \\
s\left(x, y ; \bar{x}, \sigma_{x}, \bar{y}, \sigma_{y}, c\right)= \\
\exp \left(-\frac{(x-\bar{x})^{2}}{2 \sigma_{x}^{2}}\right) \exp \left(-\frac{(y-\bar{y})^{2}}{2\left(\sigma_{y}+c\left((x-\bar{x}) / \sigma_{x}\right)^{2}\right)^{2}}\right) .
\end{gathered}
$$

Given the component shapes defined in Equations 6.3-6.5, the complete signal PDF is

$$
\begin{aligned}
\mathcal{S}(m, \Delta m) & =f_{s 1} \cdot s_{1} \\
& +\left(1-f_{s 1}\right) \cdot\left\{f_{s 2} \cdot s_{2}+\left(1-f_{s 2}\right) \cdot\left[f_{s 3} \cdot s_{3}+\left(1-f_{s 3}\right) \cdot s_{4}\right]\right\}
\end{aligned}
$$




$$
\begin{gathered}
\text { with parameters } f_{s 1}, f_{s 2}, f_{s 3}, \text { and where } \\
s_{1}=s\left(m, \Delta m ; \bar{m}_{1}, \sigma_{m 1}, \overline{\Delta m}_{1}, \sigma_{\Delta m 1}, c_{1}\right) \\
s_{2}=s\left(m, \Delta m ; \bar{m}_{2}, \sigma_{m 2}, \overline{\Delta m}_{2}, \sigma_{\Delta m 2}, c_{2}\right) \\
s_{3}=s\left(m, \Delta m ; \bar{m}_{3}, \sigma_{m 3}, \overline{\Delta m}_{3}, \sigma_{\Delta m 3}, c_{3}\right) \\
s_{4}=g\left(m ; \bar{m}_{4}, \sigma_{m 4}\right) \times c b_{s}\left(\Delta m ; \overline{\Delta m}_{4}, \sigma_{\Delta m 4}, \alpha_{4}\right) .
\end{gathered}
$$

\subsubsection{Non-Peaking Background}

The background category that does not peak in either the $m_{K \pi \pi^{0}}$ or $\Delta m$ distributions, also referred to as the combinatoric category, describes uncorrelated tracks and clusters that have been combined to make a candidate. The $\Delta m$ distribution is described using

$$
a(x ; c)=x \sqrt{\left(x / x_{0}\right)^{2}-1} \exp \left(-c\left(\left(x / x_{0}\right)^{2}-1\right)\right),
$$

where $x_{0}=0.13957 \mathrm{GeV} / c^{2}$ is the kinematic endpoint. Since the right-sign and wrong-sign distributions have different contributions from non-peaking sources, the two samples will in general have different values of $c$, which determines the specific shape. The $m_{K \pi \pi^{0}}$ distribution is described by a straight line. The complete combinatoric PDF is

$$
\mathcal{C}(m, \Delta m)=\left(1+b_{1}\left(m-1.865 \mathrm{GeV} / c^{2}\right)\right) \times a(\Delta m ; c) .
$$




\subsection{3 $m_{K \pi \pi^{0}}$ Peaking Background}

The background category that peaks in the $m_{K \pi \pi^{0}}$ distribution, also referred to as the bad- $D^{*+}$ category ${ }^{1}$, describes candidates with a signal $D^{0}$ decay but with an uncorrelated $\pi_{s}^{ \pm}$. The peaking shape is therefore the same as for the signal category, and the shape used is a one-dimensional version of Equation 6.6. This background is particularly problematic for the wrong-sign sample, since there are more candidates in this category than in the signal category. Because this background consists of real right-sign $D^{0}$ decays, distributions with structure that are different between the right-sign and wrong-sign samples are difficult to interpret. Two important examples are the decay-time distributions and the Dalitz plots. A procedure for reducing this background is discussed in Chapter 7. The complete bad- $D^{*+} \mathrm{PDF}$ is

$$
\begin{aligned}
\mathcal{F}(m, \Delta m) & =f_{s 1} \cdot s_{1 D, 1}+\left(1-f_{s 1}\right) \cdot\left\{f_{s 2} \cdot s_{1 D, 2}\right. \\
& \left.+\left(1-f_{s 2}\right) \cdot\left[f_{s 3} \cdot s_{1 D, 3}+\left(1-f_{s 3}\right) \cdot s_{1 D, 4}\right]\right\} \times a(\Delta m ; c)
\end{aligned}
$$

\footnotetext{
${ }^{1}$ In previous $D$-mixing analyses, this category has also been called the fake slow $\pi$ category.
} 
with parameters $f_{s 1}, f_{s 2}, f_{s 3}$, and where

$$
\begin{aligned}
& s_{1 D, 1}=g\left(m ; \bar{m}_{1}, \sigma_{m 1}\right) \\
& s_{1 D, 2}=g\left(m ; \bar{m}_{2}, \sigma_{m 2}\right) \\
& s_{1 D, 3}=g\left(m ; \bar{m}_{3}, \sigma_{m 3}\right) \\
& s_{1 D, 4}=g\left(m ; \bar{m}_{4}, \sigma_{m 4}\right) .
\end{aligned}
$$

\subsection{4 $\Delta m$ Peaking Background}

The background category that peaks in the $\Delta m$ distribution, also referred to as the bad- $D^{0}$ category, describes candidates from several distinct sources. First, there are candidates comprising a combinatoric $D^{0}$ candidate and a $\pi_{s}^{ \pm}$that came from a real $D^{*+}$ decay. This background does not peak sharply, and it can be described with an Argus shape (Equation 6.7). As a practical matter, this background is absorbed into the combinatoric category instead of introducing another Argus shape. Second, there are candidates comprising a real $D^{0}$ and a real $\pi_{s}^{ \pm}$, both from a $D^{*+}$ decay, but the $D^{0}$ has been reconstructed badly. An example of this would be $D^{0} \rightarrow K^{-} \pi^{+} \pi^{0} \pi^{0}$ where one of the $\pi^{0}$ candidates is missing. This background does not peak as sharply as the signal category, but it is narrow enough to require a Gaussian-like shape instead of an Argus shape. Finally, the wrong-sign sample contains a small level of doubly 
misidentified tracks, where the $K^{-}$has been called a $\pi^{-}$and the $\pi^{+}$has been called a $K^{+}$.

The peaking $\Delta m$ shape is

$$
\begin{gathered}
c b_{b}(x ; \bar{x}, \sigma, \alpha)=\left\{\begin{array}{cc}
\exp \left(-\frac{(x-\bar{x})^{2}}{2 \sigma^{2}}\right) & \text { if } \frac{(x-\bar{x})}{\sigma}<\alpha \\
a\left(b+\frac{(x-\bar{x})}{\sigma}\right)^{-1} & \text { if } \frac{(x-\bar{x})}{\sigma} \geq \alpha
\end{array}\right. \\
\alpha>0, \quad a=(1 / \alpha) \exp \left(\alpha^{2} / 2\right), \quad b=(1 / \alpha)-\alpha
\end{gathered}
$$

and the complete bad- $D^{0} \mathrm{PDF}$ is

$$
\mathcal{B}(m, \Delta m)=\left(1+b_{1}\left(m-1.865 \mathrm{GeV} / c^{2}\right)\right) \times c b_{b}\left(\Delta m ; \overline{\Delta m}_{1}, \sigma_{B}, \alpha_{B}\right)
$$

\subsection{Fit Results}

The final fit of the combined PDF described above is shown compared with the data in Figures 6.3-6.14. In order to evaluate the quality of the fit, consider the quantity, calculated for each bin in a histogram,

$$
\pm \sqrt{\chi_{i}^{2}} \equiv \frac{N_{i}-\mathrm{PDF}_{i}}{\sqrt{N_{i}}}
$$

where $i$ spans the bins in the histogram, $N_{i}$ is the number of events in the $i^{\text {th }}$ bin, and $\mathrm{PDF}_{i}$ is the integral of the probability density function over that bin. The PDF is normalized to the total number of events in the data set. The fit quality is evaluated by considering the histogram of $\pm \sqrt{\chi^{2}}$ values. Such a histogram reveals any systematic 
variations between the fitted PDF and the data. A high-quality fit will have minimal significant systematic variations.

The numbers of fitted signal events are found in Table 6.1.

Table 6.1: Results of the maximum likelihood fit to data.

wrong-sign signal events: $\quad(2.09 \pm 0.08) \times 10^{3}$

right-sign signal events: $\quad(1.0023 \pm 0.0011) \times 10^{6}$

$R \equiv \frac{\Gamma\left(D^{0} \rightarrow K^{+} \pi^{-} \pi^{0}\right)}{\Gamma\left(D^{0} \rightarrow K^{-} \pi^{+} \pi^{0}\right)}: \quad(0.208 \pm 0.008) \%$ 

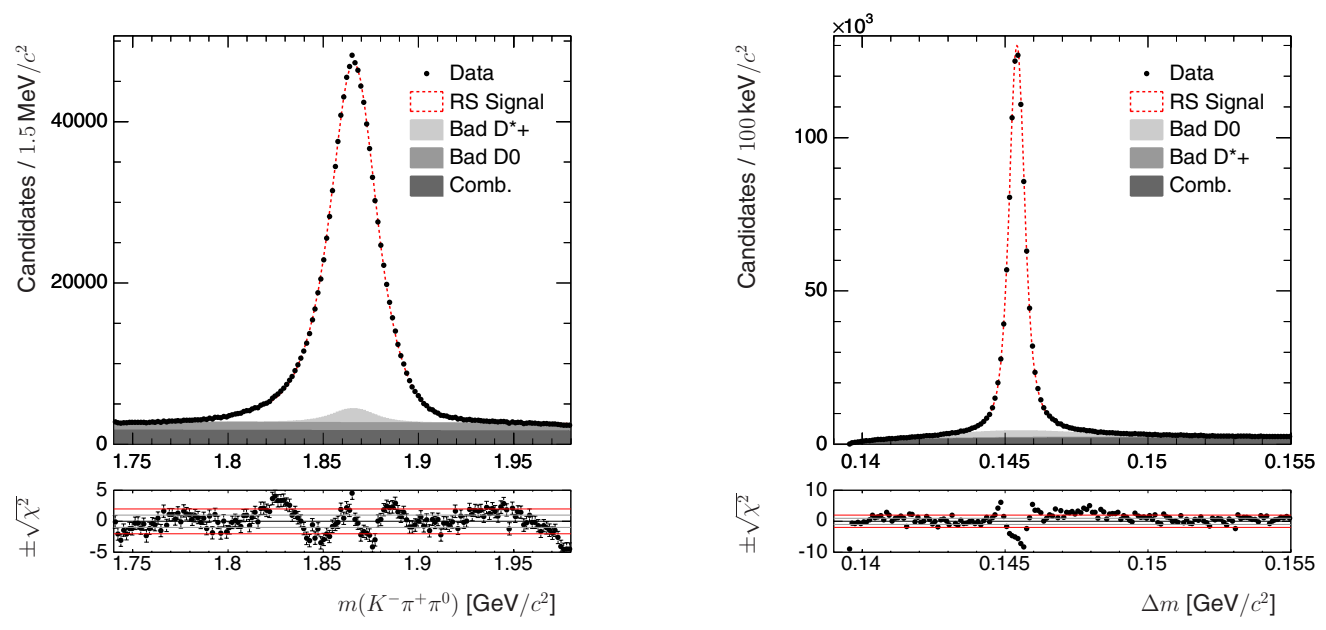

Figure 6.3: Projections of the maximum likelihood fit to all the right-sign data onto $m_{K \pi \pi^{0}}$ (left) and $\Delta m$ (right).
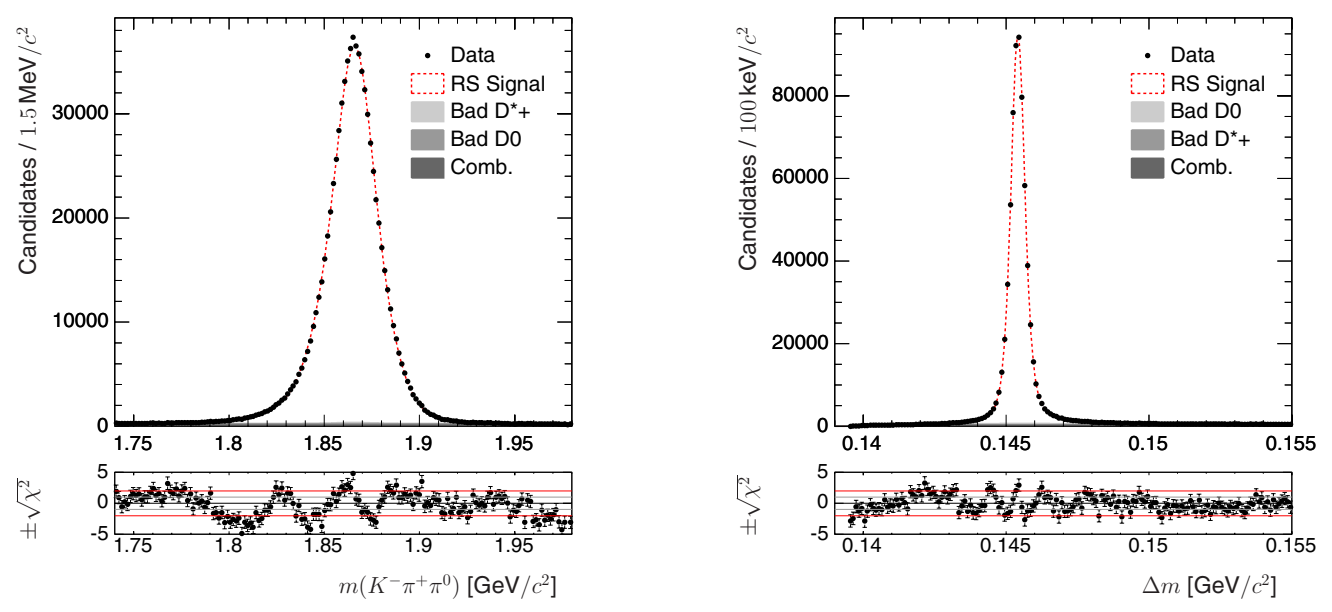

Figure 6.4: Projections of the maximum likelihood fit to all the right-sign data in the signal region onto $m_{K \pi \pi^{0}}$ (left) and $\Delta m$ (right). The $m_{K \pi \pi^{0}}$ projection requires $0.145<\Delta m<0.146 \mathrm{GeV} / c^{2}$, and the $\Delta m$ projection requires $1.85<m_{K \pi \pi^{0}}<$ $1.88 \mathrm{GeV} / c^{2}$. 

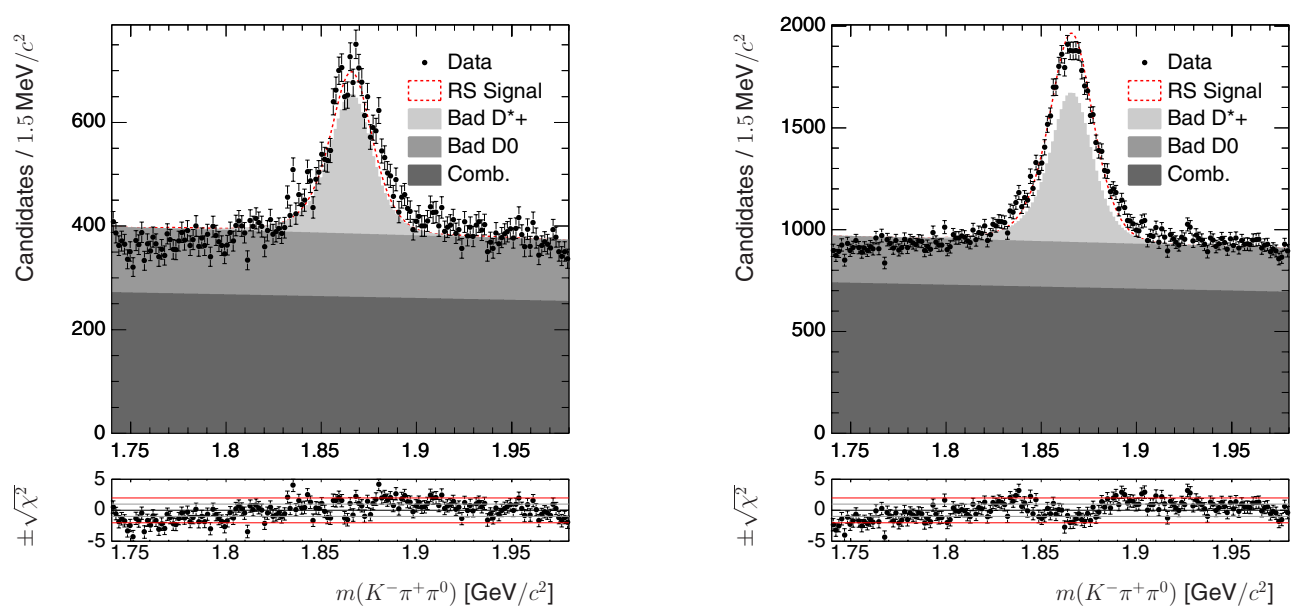

Figure 6.5: Projection of the maximum likelihood fit to all the right-sign data onto $m_{K \pi \pi^{0}}$ in the far-sideband regions of the $\Delta m$ distribution. The left sideband (left) requires $0.13957<\Delta m<0.143 \mathrm{GeV} / c^{2}$, and the right sideband (right) requires $0.149<\Delta m<0.155 \mathrm{GeV} / c^{2}$.
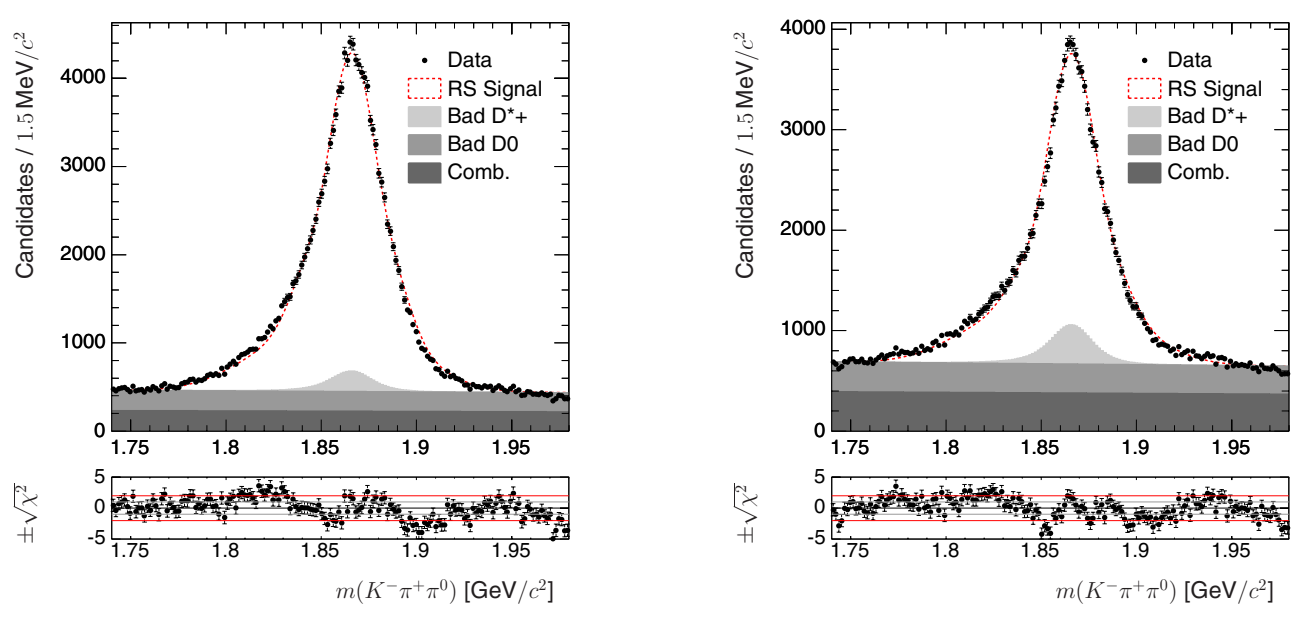

Figure 6.6: Projection of the maximum likelihood fit to all the right-sign data onto $m_{K \pi \pi^{0}}$ in the near-sideband regions of the $\Delta m$ distribution. The left sideband (left) requires $0.143<\Delta m<0.145 \mathrm{GeV} / c^{2}$, and the right sideband (right) requires $0.146<\Delta m<0.149 \mathrm{GeV} / c^{2}$. 

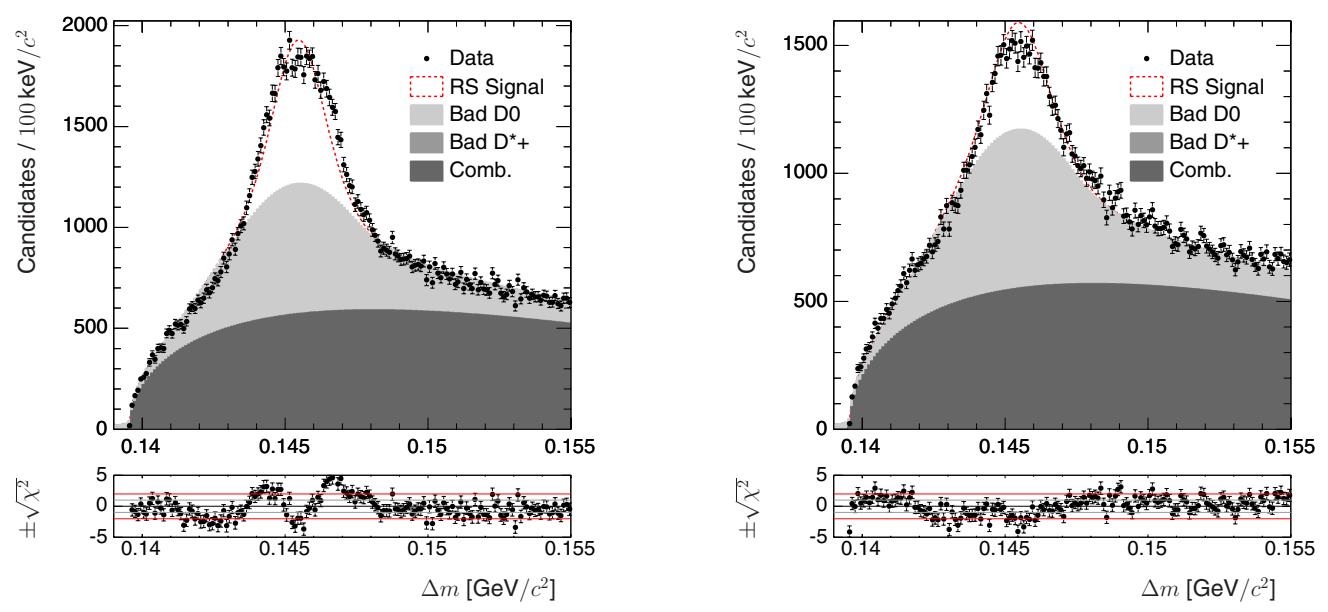

Figure 6.7: Projection of the maximum likelihood fit to all the right-sign data onto $\Delta m$ in the far-sideband regions of the $m_{K \pi \pi^{0}}$ distribution. The left sideband (left) requires $1.74<m_{K \pi \pi^{0}}<1.81 \mathrm{GeV} / c^{2}$, and the right sideband (right) requires $1.91<$ $m_{K \pi \pi^{0}}<1.98 \mathrm{GeV} / c^{2}$.
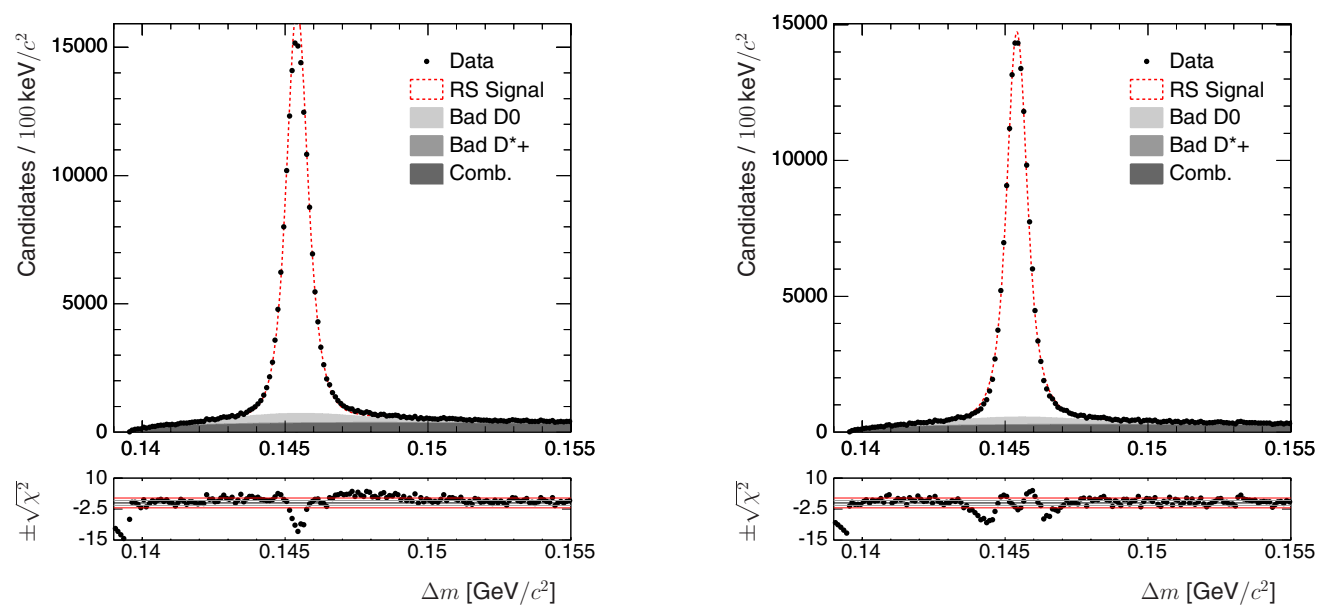

Figure 6.8: Projection of the maximum likelihood fit to all the right-sign data onto $\Delta m$ in the near-sideband regions of the $m_{K \pi \pi^{0}}$ distribution. The left sideband (left) requires $1.81<m_{K \pi \pi^{0}}<1.85 \mathrm{GeV} / c^{2}$, and the right sideband (right) requires $1.88<$ $m_{K \pi \pi^{0}}<1.91 \mathrm{GeV} / c^{2}$. 

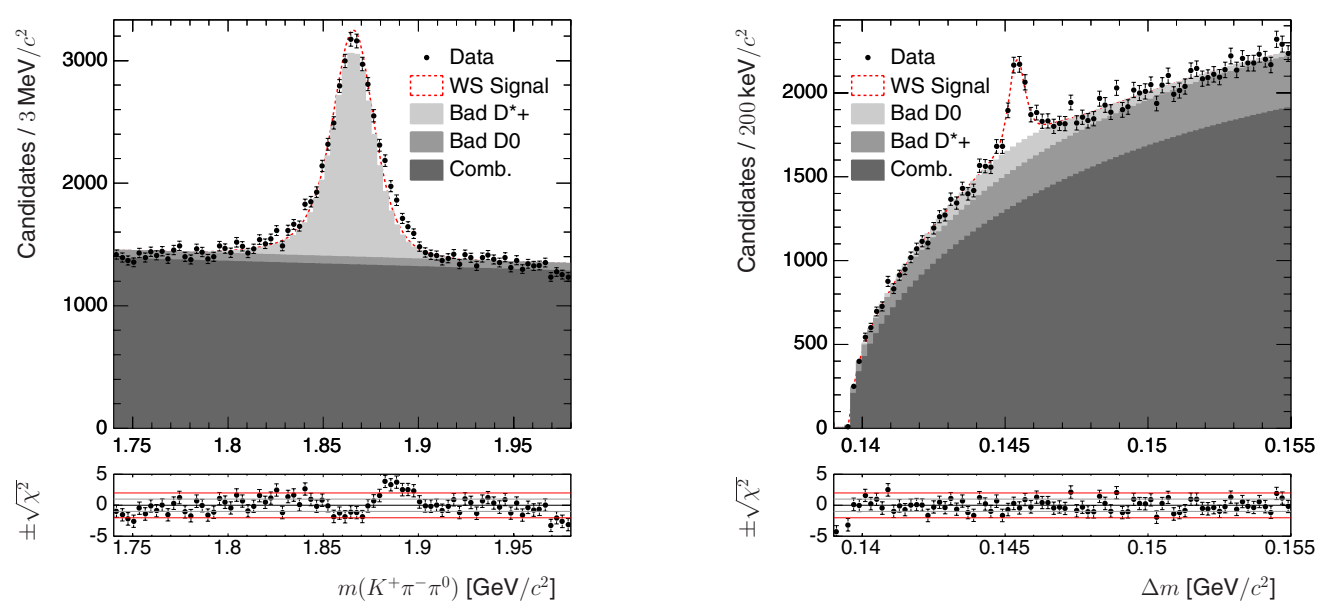

Figure 6.9: Projections of the maximum likelihood fit to all the wrong-sign data onto $m_{K \pi \pi^{0}}$ (left) and $\Delta m$ (right).
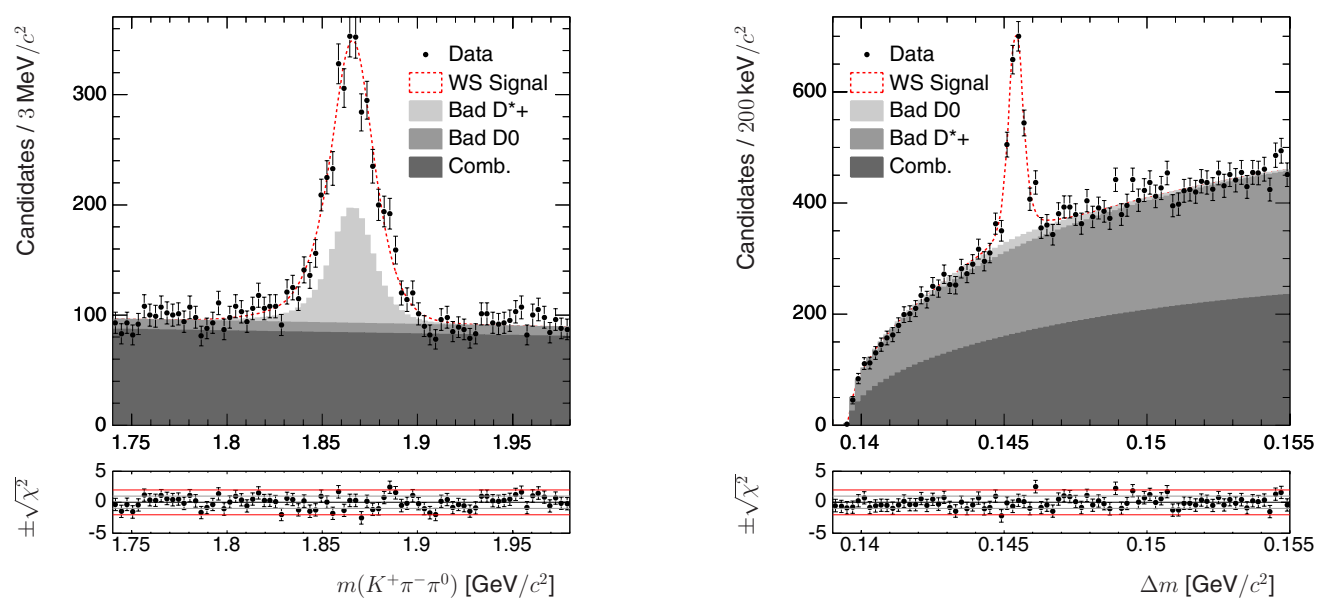

Figure 6.10: Projections of the maximum likelihood fit to all the wrong-sign data in the signal region onto $m_{K \pi \pi^{0}}$ (left) and $\Delta m$ (right). The $m_{K \pi \pi^{0}}$ projection requires $0.145<\Delta m<0.146 \mathrm{GeV} / c^{2}$, and the $\Delta m$ projection requires $1.85<m_{K \pi \pi^{0}}<$ $1.88 \mathrm{GeV} / c^{2}$. 

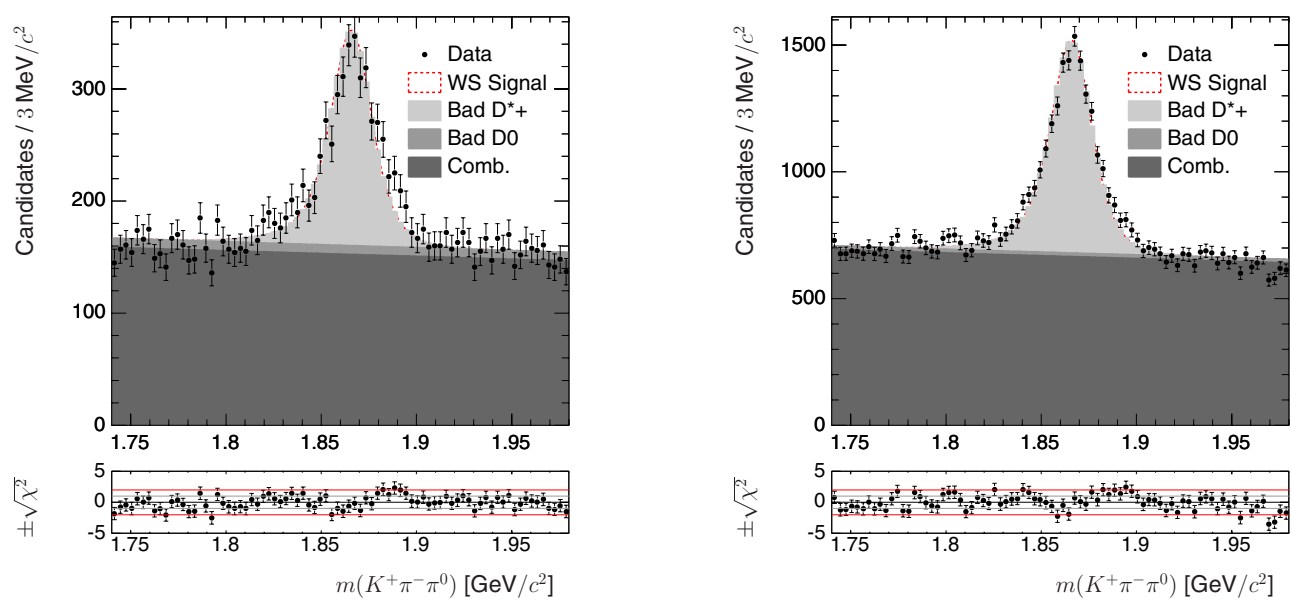

Figure 6.11: Projection of the maximum likelihood fit to all the wrong-sign data onto $m_{K \pi \pi^{0}}$ in the far-sideband regions of the $\Delta m$ distribution. The left sideband (left) requires $0.13957<\Delta m<0.143 \mathrm{GeV} / c^{2}$, and the right sideband (right) requires $0.149<\Delta m<0.155 \mathrm{GeV} / c^{2}$.
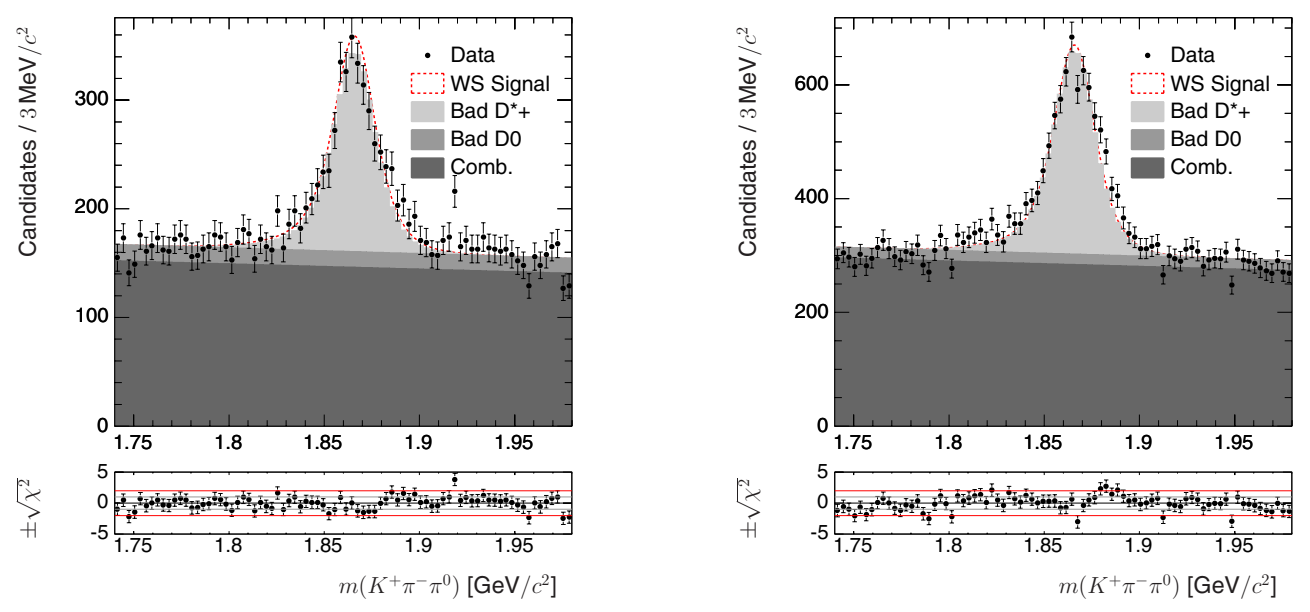

Figure 6.12: Projection of the maximum likelihood fit to all the wrong-sign data onto $m_{K \pi \pi^{0}}$ in the near-sideband regions of the $\Delta m$ distribution. The left sideband (left) requires $0.143<\Delta m<0.145 \mathrm{GeV} / c^{2}$, and the right sideband (right) requires $0.146<\Delta m<0.149 \mathrm{GeV} / c^{2}$. 

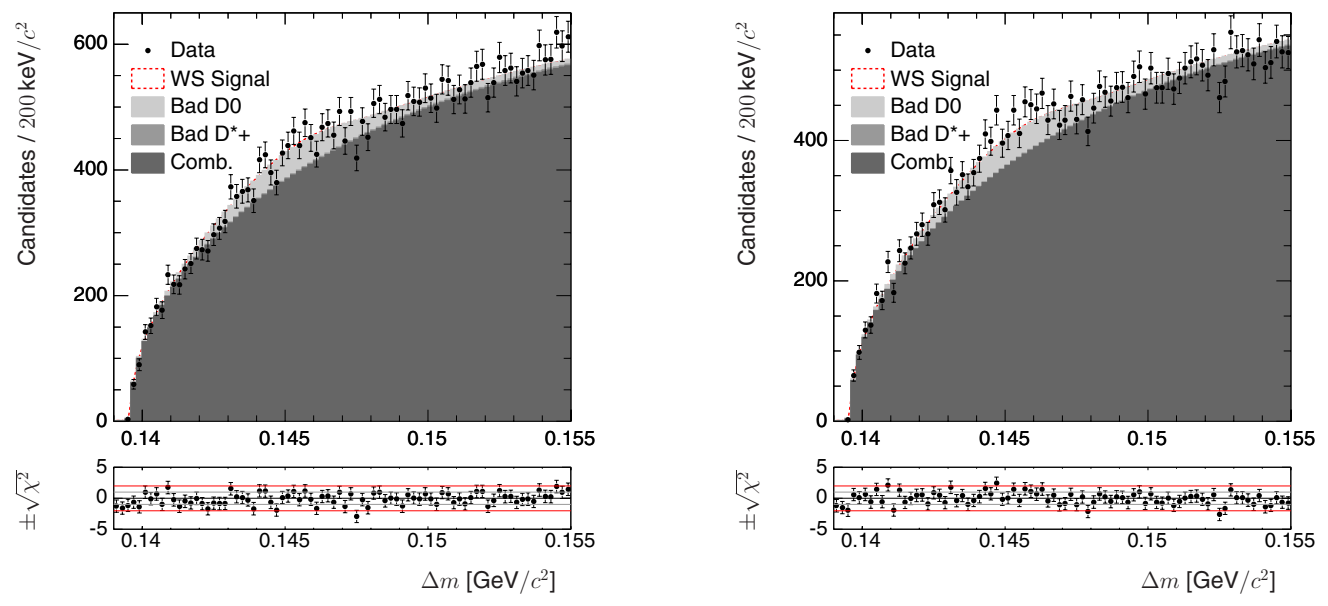

Figure 6.13: Projection of the maximum likelihood fit to all the wrong-sign data onto $\Delta m$ in the far-sideband regions of the $m_{K \pi \pi^{0}}$ distribution. The left sideband (left) requires $1.74<m_{K \pi \pi^{0}}<1.81 \mathrm{GeV} / c^{2}$, and the right sideband (right) requires $1.91<m_{K \pi \pi^{0}}<1.98 \mathrm{GeV} / c^{2}$.
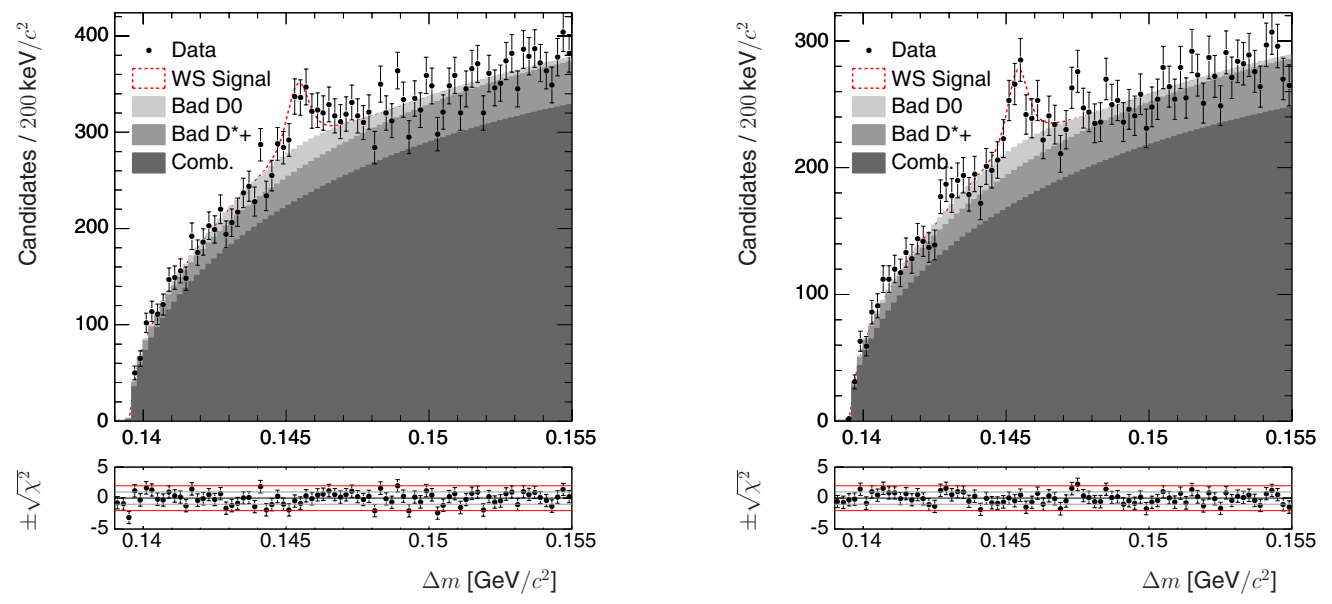

Figure 6.14: Projection of the maximum likelihood fit to all the wrong-sign data onto $\Delta m$ in the near-sideband regions of the $m_{K \pi \pi^{0}}$ distribution. The left sideband (left) requires $1.81<m_{K \pi \pi^{0}}<1.85 \mathrm{GeV} / c^{2}$, and the right sideband (right) requires $1.88<m_{K \pi \pi^{0}}<1.91 \mathrm{GeV} / c^{2}$. 


\section{Chapter 7}

\section{Event-Level Flavor Tagging}

\subsection{Motivation for a Second Flavor Tag}

While the event-selection procedure described in Chapter 4 results in a clean sample of right-sign $D^{0} \rightarrow K^{-} \pi^{+} \pi^{0}$ decays, the wrong-sign $D^{0} \rightarrow K^{+} \pi^{-} \pi^{0}$ sample still has a relatively small signal-to-background ratio. This does not pose a problem for determining the wrong-sign decay rate since the maximum likelihood fit can accurately separate the signal from the background. However, because the wrong-sign background is predominantly $D^{0} \rightarrow K^{-} \pi^{+} \pi^{0}$ decays associated with an uncorrelated $\pi^{\mp}$, distributions that have structure become hard to interpret. Two classes of observables of particular interest for a $D$-mixing search are the decay-time distribution and the resonance contributions. The wrong-sign decay-time distribution is largely popu- 
lated with real $D^{0}$ decays that tend to obscure the small deviation from an exponential shape characteristic of mixing. The wrong-sign resonance contributions will show significant structure from mis-tagged right-sign decays, and this structure is the same as that from mixed events. Since a goal of this analysis is to use the Dalitz distributions of the two decays to enhance the sensitivity to $D$ mixing, it is important to eliminate as much structured background due to right-sign decays as possible.

In the decay reconstruction, the charge of the (soft) $\pi_{s}^{ \pm}$from the $D^{*+}$ is used to tag the $D^{0}$ decay as either right-sign or wrong-sign. The following sections describe methods for using information in the event hemisphere opposite to the one in which the $D^{*+}$ is reconstructed to provide a second, event-level flavor tag. This second tag provides a consistency check on the $\pi_{s}^{ \pm}$tag in order to remove mis-tagged rightsign decays from the wrong-sign distributions. Because the wrong-sign sample has relatively low statistics, an emphasis is placed on preserving signal efficiency at the cost of keeping some level of tag impurity.

The various flavor-tag efficiencies described below were tuned and evaluated using the standard measure $S / \sqrt{S+B}$ on wrong-sign events in data. The signal $(S)$ and background $(B)$ levels were extracted from maximum likelihood fits to the tagged data sets. The background considered is only the bad- $D^{*+}$ category (see Section 6.2.3). This is the category of background events that peaks in the $m_{K \pi \pi^{0}}$ distribution and the suppression of which is the primary goal of using an event tag. Using wrong-sign 
data for tuning the flavor tags will not introduce a bias in an analysis that depends on the decay-time or Dalitz distributions as long as those distributions are not considered (even indirectly) during the tuning procedure. The final criteria were chosen to yield a tagged data set with $S / \sqrt{S+B}$ comparable to its value before tagging.

\section{2 $\pi_{\text {opp }}^{\mp}$ Tag Selection}

The $\pi_{\mathrm{opp}}^{\mp}$ tag assumes that given a reconstructed $D^{*+}$ candidate, the opposite hemisphere of the event contains an unreconstructed $D^{*-}$ candidate. A search is made for the $\pi^{-}$coming from that decay. Because half of the event has already been reconstructed as containing a $D^{*+}$, we can make a good guess as to the direction and magnitude of the expected $D^{*-}$. Also, because we expect to make a better assumption about the direction of the expected $D^{*-}$ momentum, compared to the magnitude of the $D^{*-}$ momentum, the $\pi_{\mathrm{opp}}^{\mp}$ momentum in the assumed $D^{*-}$ rest frame is projected onto the axes parallel to and orthogonal to the $D^{*-}$ momentum direction. The momentum of the $\pi^{-}$in the $D^{*-}$ rest frame should be near $40 \mathrm{MeV} / c$, a relatively low value that we can use to constrain the search.

Variables used in the selection of a $\pi_{\mathrm{opp}}^{\mp}$ candidate are defined in Table 7.1. To estimate the $D^{*-}$ rest frame, $\vec{p}_{\text {est }}$, the following method is used:

1. The reconstructed $D^{*+}$ candidate is boosted to the CM frame to get $\vec{p}_{\text {reco }}$. 
Table 7.1: Definitions of $\pi_{\mathrm{opp}}^{\mp}$ variables

\begin{tabular}{|c|c|}
\hline$\vec{p}_{\text {reco }}$ & $\begin{array}{l}\text { the momentum of the reconstructed } D^{*+} \text { candidate in the center-of- } \\
\text { mass }(\mathrm{CM}) \text { frame }\end{array}$ \\
\hline$\vec{p}_{\text {est }}$ & $\begin{array}{l}\text { the estimated momentum of the assumed } D^{*-} \text { candidate in the CM } \\
\text { frame }\end{array}$ \\
\hline$x_{p}$ & $\begin{array}{l}\left(p / p_{\max }\right) \text {, where in our specific case } p=\left|\vec{p}_{\text {est }}\right| \text { and } p_{\max }= \\
4.89 \mathrm{GeV} / c \text {, the maximum possible momentum magnitude for a } \\
D^{*-} \text { in the } B A B A R \text { detector. }\end{array}$ \\
\hline$p\left(\chi^{2}\right)$ & the $\chi^{2}$ probability of the track fit to the $D^{*+}$ vertex \\
\hline$\left|\vec{p}_{\mathrm{DS}}\right|$ & the momentum of the $\pi_{\text {opp }}^{\mp}$ candidate in the $D_{\text {est. }}^{* \mp}$ rest frame \\
\hline$p$ & shorthand for $\left|\vec{p}_{\mathrm{DS}}\right|$ \\
\hline$p_{\perp}$ & the magnitude of $\vec{p}_{\mathrm{DS}}$ transverse to the $\vec{p}_{\text {est }}$ boost \\
\hline$p_{\|}$ & the $\vec{p}_{\mathrm{DS}}$ component parallel to the $\vec{p}_{\mathrm{est}}$ boost \\
\hline
\end{tabular}

2. The direction of $\vec{p}_{\text {est }}$ is assumed to be opposite that of $\vec{p}_{\text {reco }}$ (see Figure 7.1).

3. $\left|\vec{p}_{\text {reco }}\right|$ is assumed to be near the average $x_{p}$ of the fragmentation function. We use $x_{p}=0.6$.

4. Using the assumed direction and magnitude, we construct $\vec{p}_{\text {est }}$.

A $\pi_{\text {opp }}^{\mp}$ candidate is selected by iterating over a list of tracks. Each track is refit so that it intersects the vertex of the reconstructed $D^{*+}$ candidate. The track is boosted to the estimated $D^{*-}$ rest frame. We define $\vec{p}_{\mathrm{DS}}$ as the track momentum in the $D^{*-}$ rest frame. The following selection criteria are required: 


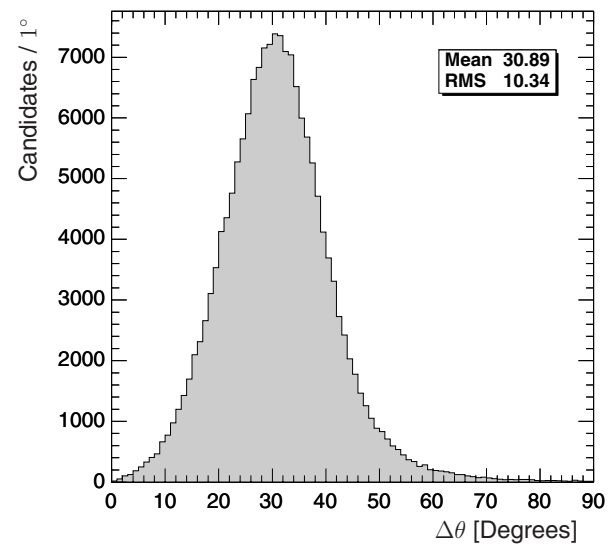

Figure 7.1: Angular difference between the estimated $D^{*-}$ direction and the true $D^{*-}$ direction in Monte Carlo events, $\cos (\Delta \theta) \equiv\left(\vec{p}_{\text {est }} \cdot \vec{p}_{\text {true }}\right) /\left(\left|\vec{p}_{\text {est }}\right|\left|\vec{p}_{\text {true }}\right|\right)$. This angular difference indicates the accuracy of the assumption that the $D^{*-}$ flies in the opposite direction from the reconstructed $D^{*+}$ (in the center-of-mass frame) when searching for $\pi_{\text {opp }}^{\mp}$ candidates in the opposite hemisphere. Only events for which the $\pi_{\text {opp }}^{\mp}$ candidate originated from a real $D^{* \mp}$ are shown.

- Input candidates are selected from the GoodTracksVeryLoose list (see Appendix A)

- $p\left(\chi^{2}\right)>0.001$

- $p_{\perp}<80 \mathrm{MeV} / c$ (see Figure 7.2)

- $-80<p_{\|}<75 \mathrm{MeV} / c$ (see Figure 7.3)

- $p<90 \mathrm{MeV} / c$ (see Figure 7.4)

If more than one candidate is selected by the above requirements, then the candidate with momentum magnitude closest to the expected magnitude is chosen. 


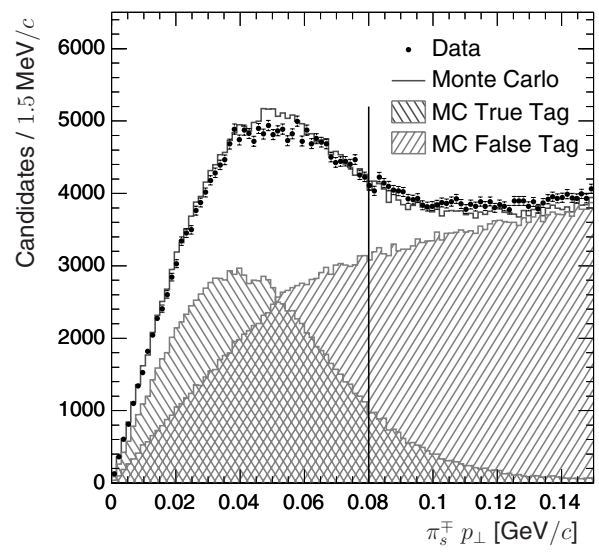

Figure 7.2: $\pi_{\mathrm{opp}}^{\mp} p_{\perp}$ distribution in data and weighted Monte Carlo for events in which a $\pi_{\text {opp }}^{\mp}$ candidate is found in the opposite hemisphere. $p_{\perp}$ is the magnitude of $\vec{p}_{\mathrm{DS}}$ transverse to the $\vec{p}_{\text {est }}$ boost. Monte Carlo truth-matched distributions illustrate events for which the $\pi_{\text {opp }}^{\mp}$ candidate originated from a real $D^{* \mp}$ (MC True Tag) and those for which the candidate did not (MC False Tag). The vertical line shows the cut on this distribution. The primary hemisphere contains decays reconstructed as $D^{*+} \rightarrow D^{0} \pi^{+}, D^{0} \rightarrow K^{-} \pi^{+} \pi^{0}$, satisfying the mass requirements $1.81<m_{K \pi \pi^{0}}<1.91 \mathrm{GeV} / c^{2}$ and $0.1444<\Delta m<0.1464 \mathrm{GeV} / c^{2}$.

\section{3 $\quad K_{\text {opp }}^{ \pm}$Tag Selection}

A $K_{\mathrm{opp}}^{ \pm}$candidate is found by looking for a high-momentum $K^{ \pm}$in the event hemisphere opposite the one in which the $D^{*+}$ is reconstructed. The $K^{+}$is assumed to come from a Cabibbo-favored decay of a $\bar{D}^{0}$ or a $D^{-}$. Because the rate of producing a wrong-charge $K^{\mp}$ from a charm decay is suppressed, and because the $K$ identification in the $B A B A R$ detector is both clean and efficient, this tag was expected to work well without imposing additional constraints. Studies confirmed this expectation, and the method for finding this candidate is simply to find the highest momentum candidate, 


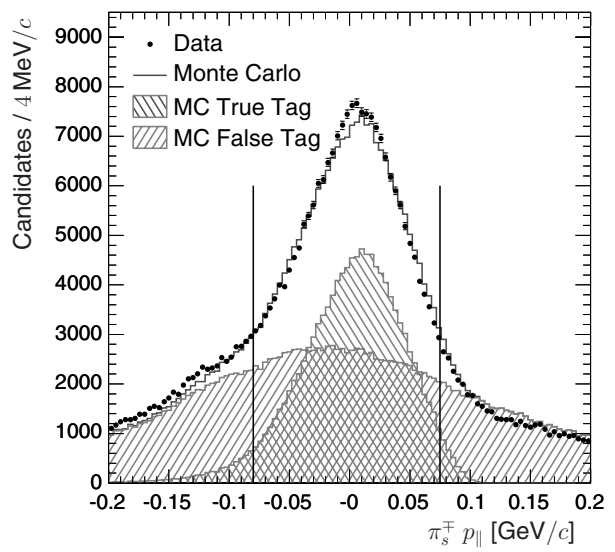

Figure 7.3: $\pi_{\mathrm{opp}}^{\mp} p_{\|}$distribution in data and weighted Monte Carlo for events in which a $\pi_{\text {opp }}^{\mp}$ candidate is found in the opposite hemisphere. $p_{\|}$is the $\vec{p}_{\mathrm{DS}}$ component parallel to the $\vec{p}_{\text {est }}$ boost. Monte Carlo truth-matched distributions illustrate events for which the $\pi_{\mathrm{opp}}^{\mp}$ candidate originated from a real $D^{* \mp}$ (MC True Tag) and those for which the candidate did not (MC False Tag). The vertical lines show the cuts on this distribution. The primary hemisphere contains decays reconstructed as $D^{*+} \rightarrow D^{0} \pi^{+}$, $D^{0} \rightarrow K^{-} \pi^{+} \pi^{0}$, satisfying the mass requirements $1.81<m_{K \pi \pi^{0}}<1.91 \mathrm{GeV} / c^{2}$ and $0.1444<\Delta m<0.1464 \mathrm{GeV} / c^{2}$.

if any, in the KLHVeryTight candidate list (see Appendix A). The center-of-mass momentum spectrum is shown in Figure 7.5.

\section{4 $e_{\text {opp }}^{\mp}$ Tag Selection}

A $e_{\mathrm{opp}}^{\mp}$ candidate is found by looking for a high-momentum $e^{\mp}$ in the event hemisphere opposite the one in which the $D^{*+}$ is reconstructed. The $e^{-}$is assumed to come from a semileptonic decay of either a $\bar{D}^{0}$ or a $D^{-}$. The following criteria are required for selecting an $e_{\mathrm{opp}}^{\mp}$ candidate: 


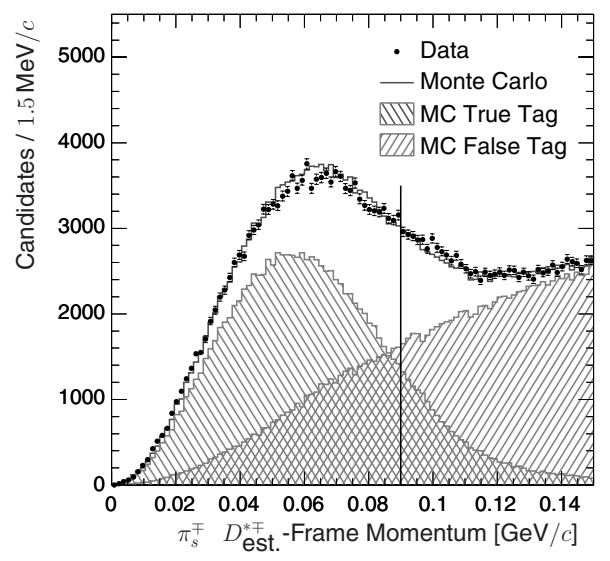

Figure 7.4: $\pi_{\mathrm{opp}}^{\mp} \vec{p}_{\mathrm{DS}}$ distribution in data and weighted Monte Carlo for events in which a $\pi_{\text {opp }}^{\mp}$ candidate is found in the opposite hemisphere. $\vec{p}_{\mathrm{DS}}$ is the $\pi_{\text {opp }}^{\mp}$ momentum in the $D_{\text {est. }}^{* \mp}$ rest frame. Monte Carlo truth-matched distributions illustrate events for which the $\pi_{\text {opp }}^{\mp}$ candidate originated from a real $D^{* \mp}$ (MC True Tag) and those for which the candidate did not (MC False Tag). The vertical line shows the cut on this distribution. The primary hemisphere contains decays reconstructed as $D^{*+} \rightarrow D^{0} \pi^{+}$, $D^{0} \rightarrow K^{-} \pi^{+} \pi^{0}$, satisfying the mass requirements $1.81<m_{K \pi \pi^{0}}<1.91 \mathrm{GeV} / c^{2}$ and $0.1444<\Delta m<0.1464 \mathrm{GeV} / c^{2}$.

- Input candidates are selected from the PidLHElectrons list (see Appendix A)

- $m^{2}>0.01\left[\mathrm{GeV} / c^{2}\right]^{2}$, where $m^{2}$ is the squared mass when the 4-momentum of the $e^{\mp}$ candidate is combined with that of another candidate on the list

If there is more than one candidate for which the above requirements are satisfied, the candidate with the highest momentum is chosen. The center-of-mass momentum spectrum is shown in Figure 7.6. 


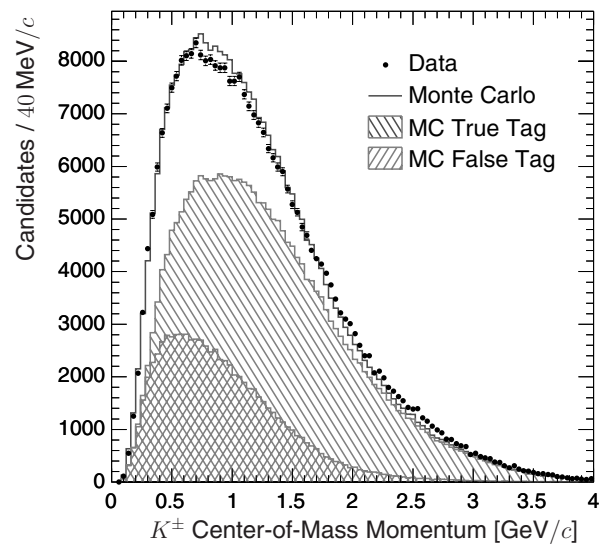

Figure 7.5: $K_{\mathrm{opp}}^{ \pm}$center-of-mass momentum distribution in data and weighted Monte Carlo for events in which a $K_{\text {opp }}^{ \pm}$candidate is found in the opposite hemisphere. Monte Carlo truth-matched distributions illustrate events for which the $K^{+}$candidate originated from a real $\bar{D}^{0}$ or $D^{-}$(MC True Tag) and those for which the candidate did not (MC False Tag). The primary hemisphere contains decays reconstructed as $D^{*+} \rightarrow D^{0} \pi^{+}, D^{0} \rightarrow K^{-} \pi^{+} \pi^{0}$, satisfying the mass requirements $1.81<m_{K \pi \pi^{0}}<1.91 \mathrm{GeV} / c^{2}$ and $0.1444<\Delta m<0.1464 \mathrm{GeV} / c^{2}$.

\section{5 $\mu_{\text {opp }}^{\mp}$ Tag Selection}

The $\mu_{\mathrm{opp}}^{\mp}$ candidate is found by looking for a high-momentum $\mu^{\mp}$ in the event hemisphere opposite the one in which the $D^{*+}$ is reconstructed. The $\mu^{-}$is assumed to come from a semileptonic decay of either a $\bar{D}^{0}$ or a $D^{-}$. The sample is made reasonably clean by simply choosing the highest momentum candidate, if any, in the muNNVeryTightFakeRate candidate list (see Appendix A). The center-of-mass momentum spectrum is shown in Figure 7.7. 


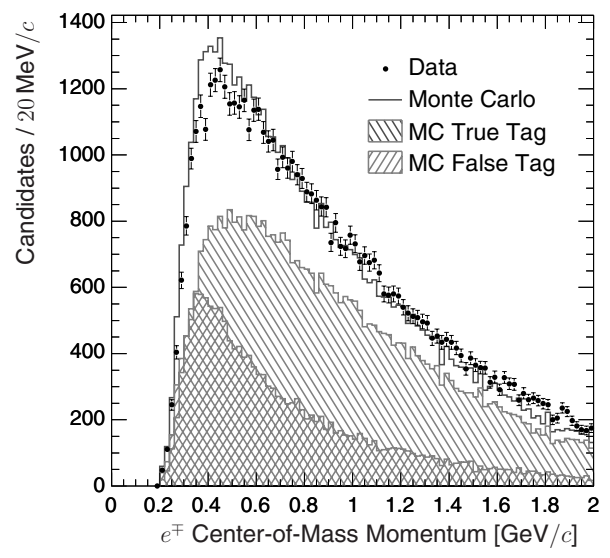

Figure 7.6: $e_{\mathrm{opp}}^{\mp}$ center-of-mass momentum distribution in data and weighted Monte Carlo for events in which a $e_{\mathrm{opp}}^{\mp}$ candidate is found in the opposite hemisphere. Monte Carlo truth-matched distributions illustrate events for which the $e^{-}$candidate originated from a real $\bar{D}^{0}$ or $D^{-}$(MC True Tag) and those for which the candidate did not (MC False Tag). The primary hemisphere contains decays reconstructed as $D^{*+} \rightarrow D^{0} \pi^{+}, D^{0} \rightarrow K^{-} \pi^{+} \pi^{0}$, satisfying the mass requirements $1.81<m_{K \pi \pi^{0}}<1.91 \mathrm{GeV} / c^{2}$ and $0.1444<\Delta m<0.1464 \mathrm{GeV} / c^{2}$.

\subsection{Tag Efficiencies}

In order to calculate the signal and background efficiencies in the wrong-sign sample for the various tags, a maximum likelihood fit is applied to the data sets using the probability density functions described in Chapter 6. The fit returns the number of events in the signal $(S)$ category and the bad- $D^{*+}(B)$ category, and using these numbers, efficiencies and the metric $S / \sqrt{S+B}$ are calculated. The fit is performed using the ranges $1.74<m_{K \pi \pi^{0}}<1.98 \mathrm{GeV} / c^{2}$ and $0.13957<\Delta m<0.155 \mathrm{GeV} / c^{2}$. The value of $S / \sqrt{S+B}$ is obtained using the ranges $1.74<m_{K \pi \pi^{0}}<1.98 \mathrm{GeV} / c^{2}$ and $0.1444<\Delta m<0.1464 \mathrm{GeV} / c^{2}$ so that only the signal region in the $\Delta m$ distribution 


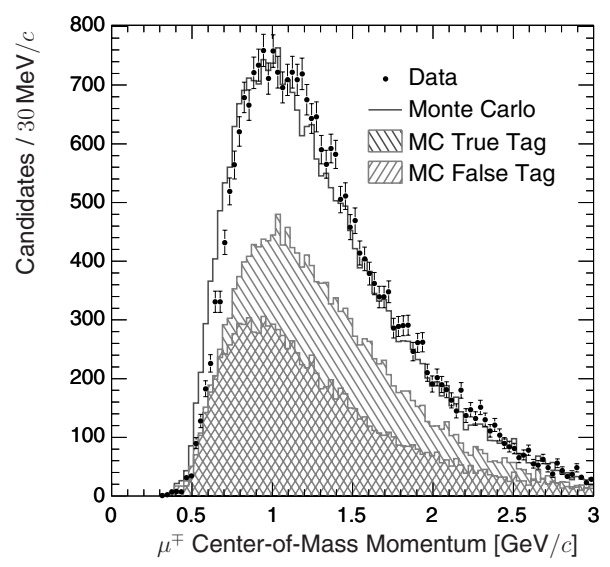

Figure 7.7: $\mu_{\mathrm{opp}}^{\mp}$ center-of-mass momentum distribution in data and weighted Monte Carlo for events in which a $\mu_{\text {opp }}^{\mp}$ candidate is found in the opposite hemisphere. Monte Carlo truth-matched distributions illustrate events for which the $\mu^{-}$candidate originated from a real $\bar{D}^{0}$ or $D^{-}$(MC True Tag) and those for which the candidate did not (MC False Tag). The primary hemisphere contains decays reconstructed as $D^{*+} \rightarrow D^{0} \pi^{+}, D^{0} \rightarrow K^{-} \pi^{+} \pi^{0}$, satisfying the mass requirements $1.81<m_{K \pi \pi^{0}}<1.91 \mathrm{GeV} / c^{2}$ and $0.1444<\Delta m<0.1464 \mathrm{GeV} / c^{2}$.

is considered.

In addition to creating event tags that are consistent with the charge of the reconstructed $D^{*+}$ candidate, the tagging methods described above can be used to create tags that are inconsistent with the reconstructed candidate. These latter tags may be used to veto events that would otherwise be accepted by one of the consistent-type tags.

Tables 7.2-7.4 list the efficiencies for each of the tags by themselves and in conjunction with various vetoes from inconsistent-type tags. Based on these findings, we choose to use the following tag combination: 
- $K_{\text {opp }}^{ \pm}$tag with a $K_{\text {opp }}^{\mp}$ veto $\left(p_{\text {veto }}>1.0 \mathrm{GeV} / c\right)$

- $\pi_{\text {opp }}^{\mp}$ tag, with a $K_{\text {opp }}^{\mp}$ veto $\left(p_{\text {veto }}>1.0 \mathrm{GeV} / c\right)$ if there is only one $K$ in the event hemisphere

- $e_{\mathrm{opp}}^{\mp} \operatorname{tag}$, with a $K_{\mathrm{opp}}^{\mp}$ veto $\left(p_{\text {veto }}>1.0 \mathrm{GeV} / c\right)$ if there is only one $K$ in the event hemisphere

- $\mu_{\mathrm{opp}}^{\mp}$ tag, with a $K_{\mathrm{opp}}^{\mp}$ veto $\left(p_{\text {veto }}>1.0 \mathrm{GeV} / c\right)$ if there is only one $K$ in the event hemisphere

The above combination of tags corresponds to the last entry in Table 7.4. The overall signal efficiency is $46.4 \%$ and the efficiency of the bad- $D^{*+}$ background category is $10.9 \%$.

The sums of the individual rates do not add up to the overall rates because some events contain more than one tag. The tagging does not provide any improvement in the right-sign sample, but it eliminates a significant amount of background from the wrong-sign sample. Figure 7.8 illustrates how this tagging method affects the $m_{K \pi \pi^{0}}$ distribution for the wrong-sign sample in data. Figure 7.9 illustrates the effect in the $\Delta m$ distribution.

Before tagging, the value of $S / \sqrt{S+B}$ is 30.7. After tagging with the combination above, the value is 27.0. There is a slight loss in the statistical significance of the signal compared with the peaking background in question. However, our interest is in 
Table 7.2: Tag efficiencies for various configurations of the $K_{\mathrm{opp}}^{ \pm}$tag and $K_{\mathrm{opp}}^{\mp}$ veto.

\begin{tabular}{|c|c|c|c|}
\hline & $\begin{array}{l}\text { signal }(S) \\
\text { efficiency }\end{array}$ & $\begin{array}{c}\text { bad- } D^{*+}(B) \\
\text { efficiency }\end{array}$ & $S / \sqrt{S+B}$ \\
\hline$K_{\mathrm{opp}}^{ \pm}$tag alone & $34.1 \%$ & $8.5 \%$ & 23.0 \\
\hline $\begin{array}{l}K_{\text {opp }}^{ \pm} \text {tag and only one } K \text { in } \\
\text { hemisphere }\end{array}$ & $29.6 \%$ & $4.3 \%$ & 22.4 \\
\hline $\begin{array}{l}K_{\text {opp }}^{ \pm} \text {tag with } K_{\text {opp }}^{\mp} \text { veto } \\
\left(p_{\text {veto }}>0.0 \mathrm{GeV} / c\right)\end{array}$ & $30.0 \%$ & $4.5 \%$ & 22.6 \\
\hline $\begin{array}{l}K_{\text {opp }}^{ \pm} \text {tag with } K_{\text {opp }}^{\mp} \text { veto } \\
\left(p_{\text {veto }}>0.5 \mathrm{GeV} / c\right)\end{array}$ & $30.7 \%$ & $5.1 \%$ & 22.6 \\
\hline $\begin{array}{l}K_{\text {opp }}^{ \pm} \text {tag with } K_{\text {opp }}^{\mp} \text { veto } \\
\left(p_{\text {veto }}>1.0 \mathrm{GeV} / c\right)\end{array}$ & $32.6 \%$ & $6.6 \%$ & 23.0 \\
\hline $\begin{array}{l}K_{\text {opp }}^{ \pm} \text {tag with } K_{\text {opp }}^{\mp} \text { veto } \\
\left(p_{\text {veto }}>1.5 \mathrm{GeV} / c\right)\end{array}$ & $33.3 \%$ & $7.7 \%$ & 22.9 \\
\hline $\begin{array}{l}K_{\text {opp }}^{ \pm} \text {tag with } K_{\text {opp }}^{\mp} \text { veto } \\
\left(p_{\text {veto }}^{\mp}>2.0 \mathrm{GeV} / c\right)\end{array}$ & $33.4 \%$ & $8.2 \%$ & 22.8 \\
\hline
\end{tabular}

Table 7.3: Tag efficiencies for various configurations of the $\pi_{\mathrm{opp}}^{\mp}$ tag and $K_{\mathrm{opp}}^{\mp}$ and $\pi_{\mathrm{opp}}^{ \pm}$ vetoes.

\begin{tabular}{lrrr}
\hline & $\begin{array}{c}\text { signal }(S) \\
\text { efficiency }\end{array}$ & $\begin{array}{c}\text { bad- } D^{*+}(B) \\
\text { efficiency }\end{array}$ & $S / \sqrt{S+B}$ \\
\hline$\pi_{\mathrm{opp}}^{\mp}$ tag alone & $13.7 \%$ & $3.7 \%$ & 14.4 \\
$\pi_{\mathrm{opp}}^{\mp}$ tag with $\pi_{\mathrm{opp}}^{ \pm}$veto & $13.2 \%$ & $3.1 \%$ & 14.4 \\
$\pi_{\mathrm{opp}}^{\mp}$ tag with $K_{\mathrm{opp}}^{\mp}$ veto & $13.3 \%$ & $3.0 \%$ & 14.5 \\
$\left(p_{\text {veto }}^{\mp}>1.0 \mathrm{GeV} / c\right)$ & & & \\
$\pi_{\mathrm{opp}}^{\mp}$ tag, with $K_{\mathrm{opp}}^{\mp}$ veto & & & \\
$\left(p_{\text {veto }}>1.0 \mathrm{GeV} / c\right)$ \\
if only one $K$ in hemisphere
\end{tabular}


Table 7.4: Tag efficiencies for various configurations of the combined tags and $K_{\mathrm{opp}}^{\mp}$ veto.

\begin{tabular}{|c|c|c|c|}
\hline & $\begin{array}{l}\text { signal }(S) \\
\text { efficiency }\end{array}$ & $\begin{array}{c}\text { bad- } D^{*+}(B) \\
\text { efficiency }\end{array}$ & $S / \sqrt{S+B}$ \\
\hline $\begin{array}{l}K_{\mathrm{opp}}^{ \pm}, \pi_{\mathrm{opp}}^{\mp}, e_{\mathrm{opp}}^{\mp} \text {, and } \mu_{\mathrm{opp}}^{\mp} \text { tags } \\
\text { with no veto }\end{array}$ & $48.3 \%$ & $13.5 \%$ & 27.0 \\
\hline $\begin{array}{ll}K_{\text {opp }}^{ \pm} \text {tag with } K_{\text {opp }}^{\mp} \text { veto } \\
\left(p_{\text {veto }}>1.0 \mathrm{GeV} / c\right) ; & \\
\pi_{\text {opp }}^{\mp}, e_{\text {opp }}^{\mp}, \text { and } \mu_{\text {opp }}^{\mp} \text { tags } & \\
\text { with } \quad K_{\text {opp }}^{\mp} & \text { veto } \\
\left(p_{\text {veto }}>1.0 \mathrm{GeV} / c\right) & \end{array}$ & $46.3 \%$ & $10.9 \%$ & 26.9 \\
\hline $\begin{array}{l}K_{\mathrm{opp}}^{ \pm} \text {tag with } K_{\mathrm{opp}}^{\mp} \text { veto } \\
\left(p_{\text {veto }}^{\mp}>1.0 \mathrm{GeV} / c\right) ; \\
\pi_{\mathrm{opp}}^{\mp}, e_{\mathrm{opp}}^{\mp}, \text { and } \mu_{\mathrm{opp}}^{\mp} \text { tags, } \\
\text { with } \quad K_{\mathrm{opp}}^{\mp} \\
\left(p_{\text {veto }}>1.0 \mathrm{GeV} / c\right) \\
\text { if only one } K \text { in hemisphere }\end{array}$ & $46.4 \%$ & $10.9 \%$ & 27.0 \\
\hline
\end{tabular}

the statistical significance of differently defined signal distributions contained within the overall wrong-sign signal category. These signal distributions, mainly the decaytime and resonance contributions, are used in a search for $D$ mixing. Therefore, by using this event-level tagging technique, we anticipate a significant gain in sensitivity to potential $D$-mixing signals. 

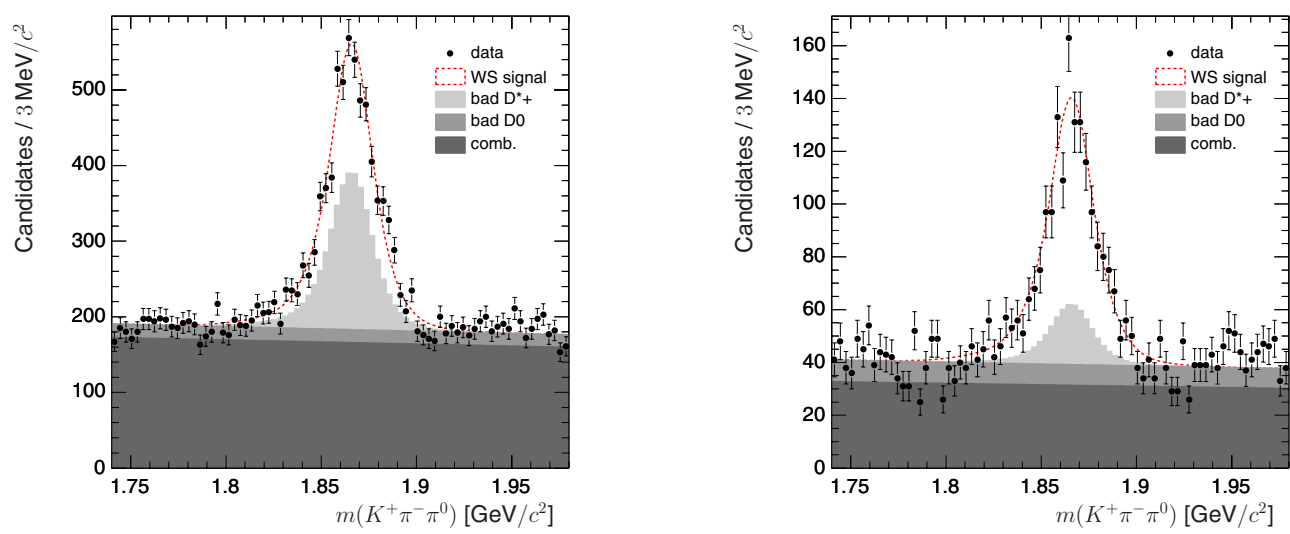

Figure 7.8: Projections onto the $m_{K \pi \pi^{0}}$ axis of the maximum likelihood fit to the wrong-sign data sample before (left) and after (right) using the combined event-level flavor tag. The shown $m_{K \pi \pi^{0}}$ projection requires $0.1444<\Delta m<0.1464 \mathrm{GeV} / c^{2}$.
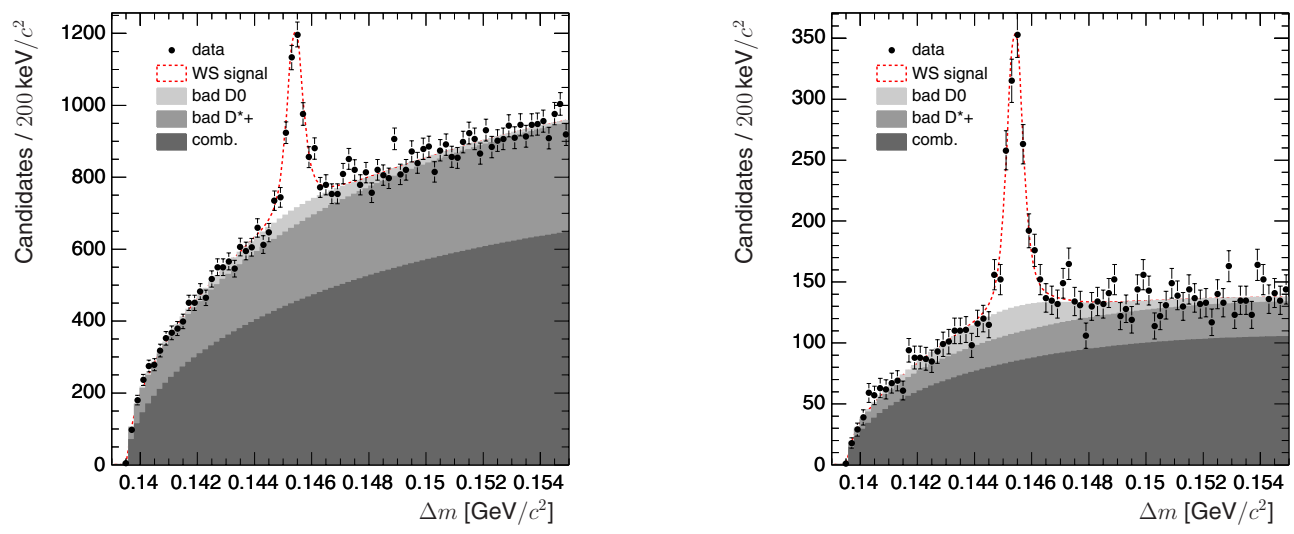

Figure 7.9: Projections onto the $\Delta m$ axis of the maximum likelihood fit to the wrongsign data sample before (left) and after (right) using the combined event-level flavor tag. The shown $\Delta m$ projection requires $1.82<m_{K \pi \pi^{0}}<1.90 \mathrm{GeV} / c^{2}$. 


\section{Chapter 8}

\section{Measurement of $D^{0} \rightarrow K^{+} \pi^{-} \pi^{0}$}

\section{Branching Ratio}

The branching ratio

$$
R \equiv \frac{\Gamma\left(D^{0} \rightarrow K^{+} \pi^{-} \pi^{0}\right)}{\Gamma\left(D^{0} \rightarrow K^{-} \pi^{+} \pi^{0}\right)}
$$

is measured using the maximum likelihood fit described in Chapter 6 with an efficiency correction based on the Dalitz-plot efficiency discussed in Chapter 5. Systematic uncertainties from the event selection, the maximum likelihood fit, and the efficiency correction are estimated, and a final result is reported. The result is validated by comparison with the result obtained using the tagged data set described in Chapter 7. 


\subsection{Dalitz-Plot Efficiency Correction}

As reported in Section 6.3, the uncorrected branching ratio determined by the maximum likelihood fit is

$$
R_{\mathrm{fit}}=(0.208 \pm 0.008) \%
$$

However, because the doubly Cabibbo-suppressed (DCS) $D^{0} \rightarrow K^{+} \pi^{-} \pi^{0}$ decays populate the Dalitz plot differently from the Cabibbo-favored (CF) $D^{0} \rightarrow K^{-} \pi^{+} \pi^{0}$ decays, the efficiencies of the two have different functions of position in the Dalitz plot.

Each candidate decay is assigned a signal weight [22]

$$
w_{s i g}\left(\vec{q}_{k}\right)=\frac{\sum_{j=1}^{4} V_{s i g, j} P_{j}\left(\vec{q}_{k}\right)}{\sum_{j=1}^{4} N_{j} P_{j}\left(\vec{q}_{k}\right)}
$$

where $j$ spans signal $(j=$ sig $=1)$ and background $(j=2,3,4)$ PDFs, $\vec{q}_{k} \equiv$ $\left(m_{K \pi \pi^{0}}, \Delta m\right)_{k}$ is the vector of dependent mass values for the $k^{\text {th }}$ decay, $P_{j}\left(\vec{q}_{k}\right)$ is the value of the $j^{\text {th }}$ PDF evaluated for the $k^{\text {th }}$ decay, $N_{j}$ is the $j^{\text {th }}$ category yield, and $V_{s i g, j}$ are the covariance matrix elements of the parameters $N_{s i g}$ and $N_{j}$. The covariance matrix $V_{i j}$ is not the full covariance matrix from the maximum likelihood fit, but rather the covariance matrix for the category yields only, given by

$$
V_{i j}^{-1}=\sum_{k} \frac{P_{i}\left(\vec{q}_{k}\right) P_{j}\left(\vec{q}_{k}\right)}{\left(\sum_{l=1}^{4} N_{l} P_{l}\left(\vec{q}_{k}\right)\right)^{2}} .
$$


These signal weights quantify the statistical separation of signal from background determined by the maximum likelihood fit, and they have the property that

$$
\sum_{k} w_{s i g}\left(\vec{q}_{k}\right)=N_{\text {sig }}
$$

The corrected signal yields for the DCS and CF decays are

$$
\sum_{k} w_{\text {sig }}\left(\vec{q}_{k}\right) / \epsilon_{k}
$$

where $\epsilon_{k}$ is the Dalitz-plot efficiency for the $k^{\text {th }}$ decay given by the fitted polynomial of Equation 5.4. Recall that efficiencies are calculated for both $D^{0} \rightarrow K^{-} \pi^{+} \pi^{0}$ and $\bar{D}^{0} \rightarrow K^{+} \pi^{-} \pi^{0}$ decays, and the appropriate efficiency is applied to the DCS or CF decay in question.

After applying the efficiency correction, we obtain the result, with statistical error only,

$$
R=(0.214 \pm 0.008) \%
$$

\subsection{Systematic Uncertainty Estimation}

The primary systematic uncertainties come from the event selection, the background model used in the maximum likelihood fit, and the efficiency correction. The uncertainties in $R$ are estimated by repeating the analysis with small changes, and taking the uncertainties to be the differences between the values of $R$ before and after the 
changes. The estimated uncertainties are listed in Table 8.1. By adding in quadrature the individual systematic uncertainties, we obtain an overall systematic uncertainty of $\pm 0.008 \%$.

Most of the uncertainties from the event selection cancel when forming the branching ratio because the reconstructed decays consist of the same particle types in equivalent decay chains. The only difference is in how the DCS decays populate the Dalitz plot compared to the CF decays, and the selection requirement with the largest effect on the Dalitz-plot efficiency is the minimum $\pi^{0}$ lab momentum requirement. This momentum requirement also reduces the background significantly. To see the effect of this requirement on both the efficiency and the background levels, the minimum $\pi^{0}$ lab momentum is lowered to $250 \mathrm{MeV} / c$ from $350 \mathrm{MeV} / c$ (see Figure 4.7), and the analysis is repeated, refitting for the signal yields and recalculating the Dalitz-plot efficiency.

The systematic uncertainty from the maximum likelihood fit is mostly due to the background shape. The signal shape, since it is used for both the $D^{0} \rightarrow K^{+} \pi^{-} \pi^{0}$ and the $D^{0} \rightarrow K^{-} \pi^{+} \pi^{0}$ decays, has no systematic uncertainty associated with it when forming the branching ratio. To estimate the uncertainty in the background, the linear background shape in the $m_{K \pi \pi^{0}}$ distribution is changed to a quadratic shape. This allows the background to absorb some signal if this would result in a larger likelihood. The background shape in the $m_{K \pi \pi^{0}}$ distribution has a greater uncertainty than 
Table 8.1: Systematic checks and uncertainties of the branching ratio measurement

\begin{tabular}{lccc}
\hline & & $\begin{array}{c}\text { Statistical } \\
\text { uncertainty }\end{array}$ & $\begin{array}{c}\text { Difference } \\
\text { from } \\
\text { primary result }\end{array}$ \\
\hline Primary result & $R$ & & \\
& $0.214 \%$ & $0.008 \%$ & \\
Selection requirement $250 \mathrm{MeV} / c<p_{\pi^{0}}$ & $0.221 \%$ & $0.009 \%$ & $0.007 \%$ \\
$2^{\text {nd }}$-order polynomial $m_{K \pi \pi^{0}}$ background & $0.211 \%$ & $0.009 \%$ & $-0.003 \%$ \\
Alternative efficiency correction & $0.211 \%$ & $0.008 \%$ & $-0.003 \%$ \\
\hline
\end{tabular}

the background in the $\Delta m$ distribution because the $m_{K \pi \pi^{0}}$ signal shape is less well known, the $m_{K \pi \pi^{0}}$ range is relatively narrow compared to the signal width, and there are a larger number of unknown backgrounds that can contribute in the $m_{K \pi \pi^{0}}$ distribution.

Finally, we investigate the uncertainty in the Dalitz-plot efficiency correction. This is done by using an alternative correction algorithm on the primary result. Instead of fitting the binned efficiency histogram to a third-order polynomial and using that polynomial to obtain the efficiency for a given point in the Dalitz plot, the efficiency is taken directly from the binned histogram. This has the effect of varying the efficiency according to Poisson statistics across the Dalitz plot, and it results in an extreme treatment of the edges of the Dalitz plot for which there are few statistics to determine the efficiency. The result is an overly conservative estimate of this systematic uncertainty.

The results of these systematic checks are given below in Table 8.1. 


\subsection{Comparison with the Tagged Data Set}

In order to validate the final branching ratio result from the full data set, we perform the analysis on the tagged data set described in Chapter 7 and check to see that the results are consistent. The tagged data set provides a meaningful check on the final result because the background distributions are so drastically reduced in size and changed in shape, yet the signal distribution shapes remain the same.

The uncorrected branching ratio from fitting the tagged data set is

$$
R_{\text {fit }}=(0.213 \pm 0.010) \%
$$

and the value after applying the efficiency correction is

$$
R=(0.220 \pm 0.010) \% \text {. }
$$

This result is indeed consistent with the primary reported value.

A comparison with simulated Monte Carlo events is not done because the fitted signal shape for Monte Carlo events is significantly different from that used for the recorded data, the $\pi^{0}$ simulation does not reproduce the recorded-data distributions, and the Dalitz-plot structure of the DCS decays is not simulated. Therefore, it is questionable how much can be learned by performing this analysis on simulated events. 


\subsection{Final Branching Ratio Result}

Combining the maximum likelihood fit, the efficiency correction, and the estimated systematic uncertainties, we find

$$
R=(0.214 \pm 0.008 \text { (stat) } \pm 0.008 \text { (syst) }) \% \text {. }
$$

Although a small number of events from $D$ mixing may contribute to this measurement of $R$, based on current experimental limits, this contribution can be at most the size of the statistical error given. As discussed in Section 2.2, the relatively small value of $R$ for this decay mode yields a greater sensitivity to $D$ mixing in a lifetime analysis. 


\section{Chapter 9}

\section{Measurements of Resonance}

\section{Contributions to $D^{0} \rightarrow K^{+} \pi^{-} \pi^{0}$}

\subsection{Introduction}

The Cabibbo-favored (CF) $D^{0} \rightarrow K^{-} \pi^{+} \pi^{0}$ decay $^{1}$ proceeds primarily through one of three intermediate resonances $K^{-} \rho^{+}, K^{*-} \pi^{+}$, and $\bar{K}^{* 0} \pi^{0}[23]$. We expect the doubly Cabibbo-suppressed (DCS) $D^{0} \rightarrow K^{+} \pi^{-} \pi^{0}$ decay to proceed through similar resonances. However, as indicated by the Feynman diagrams in Figure 2.1, we anticipate that the relative sizes of the DCS resonance amplitudes compared with the corresponding CF amplitudes will follow an approximate flavor-SU(3) symmetry. In

\footnotetext{
${ }^{1}$ Charge conjugation is implied except where otherwise stated.
} 
other words, since the CF decay is dominated by the $D^{0} \rightarrow K^{-} \rho^{+}$resonance, we expect the DCS decay to be dominated by the $D^{0} \rightarrow \pi^{-} K^{*+}$ resonance. The Dalitz plots of the CF and DCS will therefore be populated differently, and we can take advantage of this in a search for $D$ mixing by selecting Dalitz-plot regions with a relatively large population of CF decays compared to DCS decays. Recall that the arguments for selecting a sample in this manner were made in Chapter 2.

It would be very challenging to correctly determine the resonance contributions to the DCS decay without the tagged data set described in Chapter 7. Studies show that even though the size of the untagged sample is significantly larger than that of the tagged sample, the background is dominated by $\mathrm{CF}$ decays that have been associated with $\pi_{s}^{ \pm}$candidates not coming from $D^{*}$ decays. In particular, the background in the $D^{0} \rightarrow K^{+} \rho^{-}$invariant-mass distribution is so large that the maximum likelihood fit described below cannot correctly separate it from the signal. Therefore, in this chapter, only the tagged data set will be considered.

Ideally, a full Dalitz-plot analysis of the CF and DCS decays would be undertaken to determine the resonance contributions to each type of decay. Combining such an analysis with an analysis of the decay-time distribution would provide the most sensitivity to $D$ mixing. However, a time-dependent Dalitz-plot analysis is a major undertaking, and a competitive search for $D$ mixing can be performed by simply selecting regions of the Dalitz plot to analyze. In particular, we do not aim for a competitive 
analysis of the resonance contributions to the CF decay, but rather a competent and sufficient analysis to complement the analysis of the DCS decay resonance structure. The lineshapes used in this chapter to describe the CF resonances are known to be crude, but they are adequate for the purpose of this work.

For each of the three primary resonances through which the CF and DCS decays may proceed- $K \rho, K^{*} \pi$, and $K^{* 0} \pi^{0}$-we measure the corresponding branching ratio

$$
\begin{aligned}
R_{K \rho} & \equiv \frac{\Gamma\left(D^{0} \rightarrow K^{+} \rho^{-}\right)}{\Gamma\left(D^{0} \rightarrow K^{-} \rho^{+}\right)} \\
R_{K^{*} \pi} & \equiv \frac{\Gamma\left(D^{0} \rightarrow K^{*+} \pi^{-}\right)}{\Gamma\left(D^{0} \rightarrow K^{*-} \pi^{+}\right)} \\
R_{K^{* 0} \pi^{0}} & \equiv \frac{\Gamma\left(D^{0} \rightarrow K^{* 0} \pi^{0}\right)}{\Gamma\left(D^{0} \rightarrow \bar{K}^{* 0} \pi^{0}\right)}
\end{aligned}
$$

We expect these branching ratio measurements to be more accurate than the ones below because the same signal lineshape and reconstruction efficiency apply to both modes in the ratio. Therefore, systematic uncertainties related to the these two parts of the analysis cancel when forming the branching ratio. Also, we measure the relative contributions to the overall DCS decay

$$
\begin{aligned}
\mathcal{B} r\left(D^{0} \rightarrow K^{+} \rho^{-}\right) & \equiv \frac{\Gamma\left(D^{0} \rightarrow K^{+} \rho^{-}\right)}{\Gamma\left(D^{0} \rightarrow K^{+} \pi^{-} \pi^{0}\right)} \\
\mathcal{B} r\left(D^{0} \rightarrow K^{*+} \pi^{-}\right) & \equiv \frac{\Gamma\left(D^{0} \rightarrow K^{*+} \pi^{-}, K^{*+} \rightarrow K^{+} \pi^{0}\right)}{\Gamma\left(D^{0} \rightarrow K^{+} \pi^{-} \pi^{0}\right)} \\
\mathcal{B} r\left(D^{0} \rightarrow K^{* 0} \pi^{0}\right) & \equiv \frac{\Gamma\left(D^{0} \rightarrow K^{* 0} \pi^{0}, K^{* 0} \rightarrow K^{+} \pi^{-}\right)}{\Gamma\left(D^{0} \rightarrow K^{+} \pi^{-} \pi^{0}\right)}
\end{aligned}
$$


and to the overall CF decay

$$
\begin{aligned}
\mathcal{B} r\left(D^{0} \rightarrow K^{-} \rho^{+}\right) & \equiv \frac{\Gamma\left(D^{0} \rightarrow K^{-} \rho^{+}\right)}{\Gamma\left(D^{0} \rightarrow K^{-} \pi^{+} \pi^{0}\right)} \\
\mathcal{B} r\left(D^{0} \rightarrow K^{*-} \pi^{+}\right) & \equiv \frac{\Gamma\left(D^{0} \rightarrow K^{*-} \pi^{+}, K^{*-} \rightarrow K^{-} \pi^{0}\right)}{\Gamma\left(D^{0} \rightarrow K^{-} \pi^{+} \pi^{0}\right)} \\
\mathcal{B} r\left(D^{0} \rightarrow \bar{K}^{* 0} \pi^{0}\right) & \equiv \frac{\Gamma\left(D^{0} \rightarrow \overline{K^{* 0}} \pi^{0}, \bar{K}^{* 0} \rightarrow K^{-} \pi^{+}\right)}{\Gamma\left(D^{0} \rightarrow K^{-} \pi^{+} \pi^{0}\right)}
\end{aligned}
$$

These branching ratios are sensitive to both the signal lineshape and the Dalitz-plot efficiency; therefore, they have a larger systematic uncertainty. As will become apparent, the uncertainty associated with the lineshapes used in this analysis dominate the overall systematic uncertainty.

\subsection{Efficiency-Corrected Two-Body}

\section{Mass Distributions}

We begin by looking at the Cabibbo-favored Dalitz plot in Figure 9.1 and the efficiency-corrected two-body mass distributions in Figures 9.2-9.4 to get a sense of the resonance structure and the relative sizes of the resonance contributions. Using the tagged dataset fitted with the two-dimensional PDF described in Chapter 6, each event is assigned a signal weight defined in Equation 8.3. These weights are corrected for variations in the Dalitz-plot efficiency according to Equation 8.6, and the twobody mass distributions are shown after this weighting. The signal weights do not 
significantly enhance the CF distributions, but they dramatically show the otherwiseobscured DCS signal distributions. The signal weights do not accurately show the DCS signal distributions for $D^{0} \rightarrow K^{+} \rho^{-}$in the untagged dataset, again justifying the exclusive use of the tagged dataset in this chapter.

It is immediately apparent that the DCS distributions are in fact very different from the corresponding CF distributions. It can also be seen that the two-body distributions for the CF decays do not qualitatively agree with those shown in the $D^{0} \rightarrow K^{-} \pi^{+} \pi^{0}$ Dalitz-plot analysis performed by the CLEO Collaboration [24], which provides the basis for the corresponding values listed in the Review of Particle Physics (RPP) [23]. Even an exaggerated estimate of the uncertainty for the Dalitz-plot efficiency correction cannot account for the difference. Thus, we expect that an analysis of this data set will yield CF branching ratios that significantly differ from those in the RPP.

\subsection{Description of Probability Density Functions}

In order to quantitatively describe the resonance contributions to the overall $\mathrm{CF}$ and DCS decay rates, we fit the one-dimensional projections onto the corresponding invariant-mass distributions of the three primary two-body systems. If the signal contributions have been accurately corrected for Dalitz-plot efficiency variations, then any interference effects from other resonances in the kinematically allowed region will cancel in the mass projections. Furthermore, for the particular resonances in- 


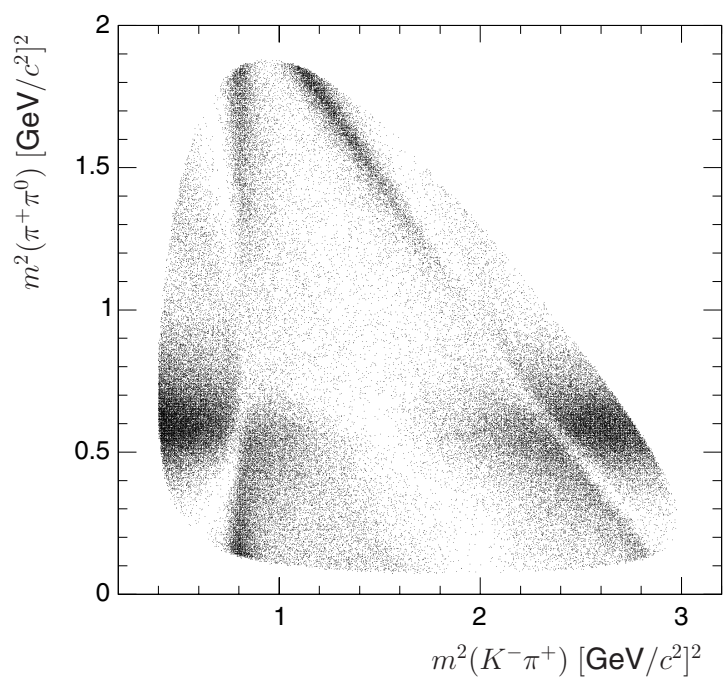

Figure 9.1: Dalitz plot of the $D^{0} \rightarrow K^{-} \pi^{+} \pi^{0}$ decay with 97,722 events selected from the tagged sample with the requirements $1.85<m_{K \pi \pi^{0}}<1.88 \mathrm{GeV} / c^{2}$ and $0.1444<$ $\Delta m<0.1464 \mathrm{GeV} / c^{2}$.
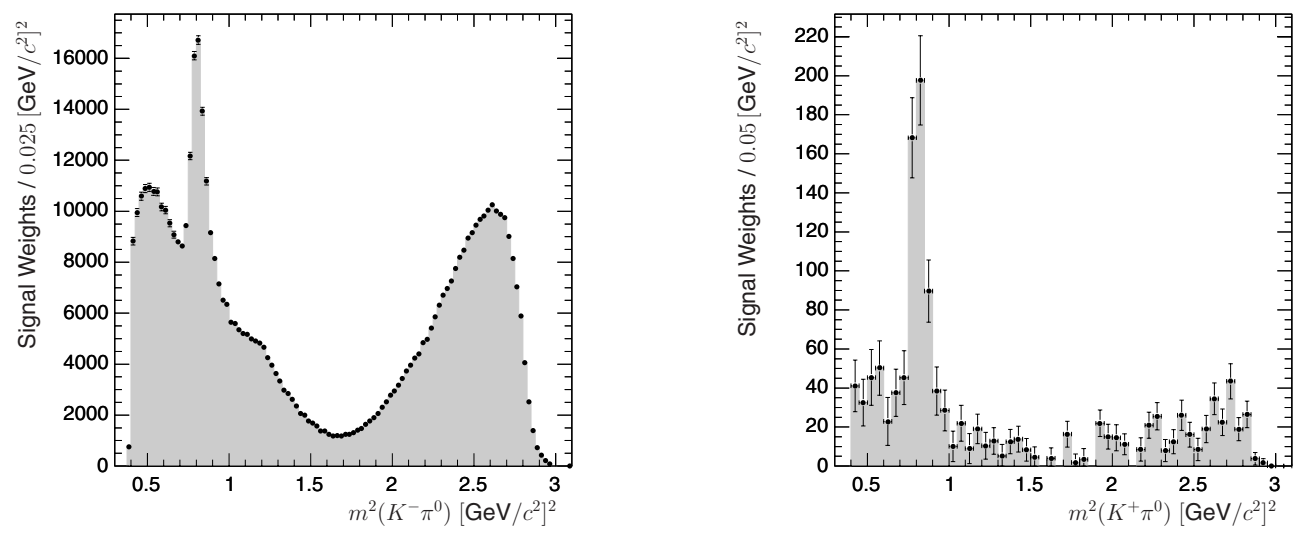

Figure 9.2: The $m^{2}\left(K^{-} \pi^{0}\right)$ and $m^{2}\left(K^{+} \pi^{0}\right)$ mass distributions, respectively, of efficiency-corrected, signal-weighted, Cabibbo-favored $D^{0} \rightarrow K^{-} \pi^{+} \pi^{0}$ candidates (left) and doubly Cabibbo-suppressed $D^{0} \rightarrow K^{+} \pi^{-} \pi^{0}$ candidates (right). 

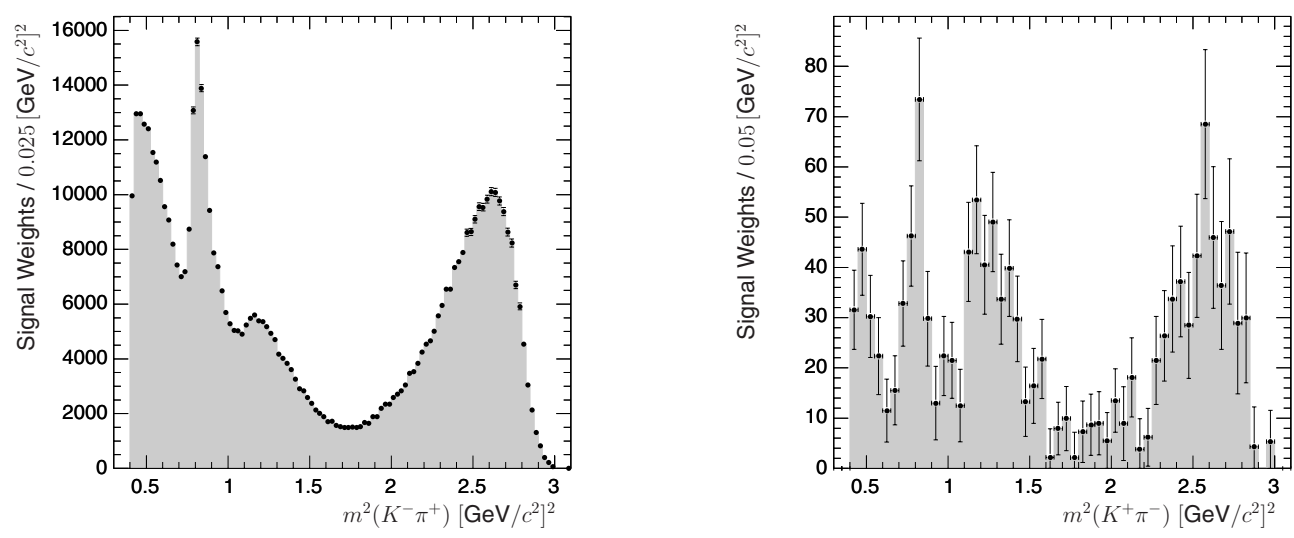

Figure 9.3: The $m^{2}\left(K^{-} \pi^{+}\right)$and $m^{2}\left(K^{+} \pi^{-}\right)$mass distributions, respectively, of efficiency-corrected, signal-weighted, Cabibbo-favored $D^{0} \rightarrow K^{-} \pi^{+} \pi^{0}$ candidates (left) and doubly Cabibbo-suppressed $D^{0} \rightarrow K^{+} \pi^{-} \pi^{0}$ candidates (right).
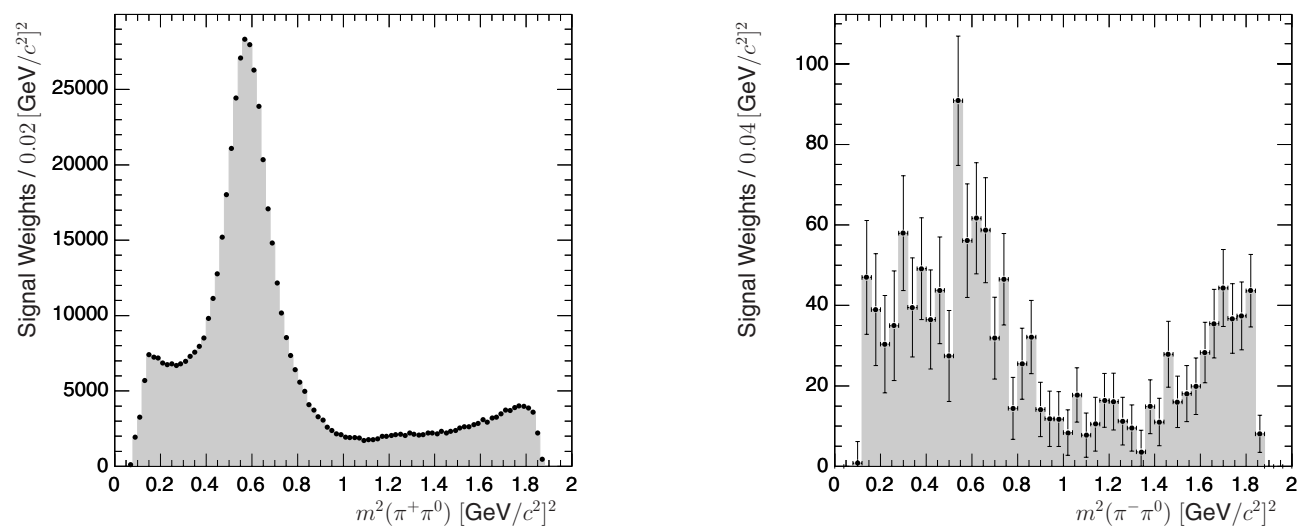

Figure 9.4: The $m^{2}\left(\pi^{+} \pi^{0}\right)$ and $m^{2}\left(\pi^{-} \pi^{0}\right)$ mass distributions, respectively, of efficiency-corrected, signal-weighted, Cabibbo-favored $D^{0} \rightarrow K^{-} \pi^{+} \pi^{0}$ candidates (left) and doubly Cabibbo-suppressed $D^{0} \rightarrow K^{+} \pi^{-} \pi^{0}$ candidates (right). 
volved in this analysis- $\rho, K^{*}$, and $K^{* 0}$ - the intrinsic width of the resonances is much larger than the detector resolution, so we may use lineshapes derived solely from the physics of the decay. However, although the resonant portion of the signal in a particular mass distribution will follow a well-defined lineshape, the non-resonant portion in that distribution is expected to vary significantly.

The analysis presented here is a drastic simplification of the above considerations, yet it appears to be adequate for the analysis of the DCS decays. For the CF decays, the analysis is too blunt for the incredibly large statistical sample available. However, since the efficiency-corrected distributions are significantly different from those used to determine the values in the RPP, the CF resonance contributions determined in this chapter may be more accurate than the RPP, regardless of the specific methods chosen to fit the distributions.

In a particular two-body mass distribution, the signal may be split into two categories: the resonant portion, and the non-resonant portion. The resonant portion is fit with a Breit-Wigner

$$
b w(m ; \bar{m}, \Gamma)=\frac{1}{(m-\bar{m})^{2}+\Gamma^{2} / 4}
$$

where $\bar{m}$ is the resonance mass and $\Gamma$ is the resonance width. In the fits performed for this chapter, the mass and width are fixed to their measured values in the RPP. This shape would be accurate for narrow resonances; however, these resonances are not narrow, and a more accurate shape would have a width that depends on $m$. The non- 
resonant portion is fit to a line, which is surely an oversimplification if one considers how the Dalitz plot is populated in Figure 9.1. In particular, the non-resonant signal contribution appears to oscillate as a function of the invariant mass due to interference effects. However, the advantages of this simple model are that it is easy to vary in order to estimate systematic effects, it tends neither to absorb nor reject resonant signal events, and it contains only one free parameter, allowing the fit to the lowstatistics DCS sample to converge reliably.

The background comprises two general types of decays: those that contain real $D^{0}$ decays associated with uncorrelated $\pi_{s}^{ \pm}$candidates, and those that do not contain real $D^{0}$ decays. The former type will have the $\mathrm{CF}$ resonant structure, and like the signal, can be split into resonant and non-resonant contributions and fit with the same lineshapes as the signal. The latter type produces a smoothly varying shape across the mass distribution, with the possibility of containing a small background resonant contribution. The latter type is fit using a second-order polynomial added to a BreitWigner.

\subsection{Fit Results and Systematic Uncertainty Estimation}

To obtain the number of signal events in a particular resonance, a maximum likelihood fit to the relevant invariant-mass distribution is performed. The likelihood fit is a three-dimensional fit to $\left\{m_{K \pi \pi^{0}}, \Delta m, m_{P P}\right\}$ with the likelihood defined by Equa- 
tion 6.1, where $m_{P P} \in\left\{m_{\pi \pi^{0}}, m_{K \pi^{0}}, m_{K \pi}\right\}$ is the invariant mass of the two pseudoscalars. The two dimensions $\left\{m_{K \pi \pi^{0}}, \Delta m\right\}$ are fit to the same probability density functions (PDFs) as in Chapter 6, and they are needed to accurately separate signal from the three background categories. The crude lineshapes used to determine the resonance contributions work primarily because the fit to $\left\{m_{K \pi \pi^{0}}, \Delta m\right\}$ is well-tuned.

The data samples used in the fits have two important features. First, each event is associated with an efficiency weight to correct for the Dalitz-plot efficiency variation, and these weights are included in the maximum likelihood fit. Since background events are also weighted, some regions of $m_{P P}$, when plotted with the fitted function, appear to have a larger background contribution than in other histograms of the same data. Second, the values of $m_{P P}$ used in the fits are calculated from a decay-chain fit similar to the one described in Section 4.3 with the additional constraint that $m_{K \pi \pi^{0}}$ is required to have the measured $D^{0}$ mass. Without this $D^{0}$-mass constraint, the centers of the signal distributions in $m_{P P}$ vary across the Dalitz plot. However, with this constraint, the signal distributions are stable, while the background varies across the Dalitz plot.

An important feature of these fits is that the background and overall signal yields are as accurately determined by the two-dimensional $\left\{m_{K \pi \pi^{0}}, \Delta m\right\}$ fit as in Chapter 8 . Thus, although the background shapes may not be completely correct in the $m_{P P}$ distribution, the yields are correct. Also, even though the relative levels of res- 
onant and non-resonant signal are completely dependent upon the $m_{P P}$ distribution, the total signal yield is correct and constrained by the other two dimensions. These constraints improve the overall reliability of the fit results.

The fit is performed in two steps. First, the two-dimensional $\left\{m_{K \pi \pi^{0}}, \Delta m\right\}$ fit is performed to adjust all the parameters and yields to the portion of the data set contained within the fit region of the $m_{P P}$ distribution. Second, the parameters included in the two-dimensional fit are fixed, and the three-dimensional fit is performed to find the best values of the parameters in the $m_{P P}$ PDFs, including the yields for the five categories: resonant signal, non-resonant signal, resonant fake- $\pi_{s}^{ \pm}$background, non-resonant fake- $\pi_{s}^{ \pm}$background, and non- $D^{0}$ background. The final fits are shown projected onto the $m_{P P}$ axes for signal regions of $\left\{m_{K \pi \pi^{0}}, \Delta m\right\}$ in Figures 9.5-9.10.

Systematic uncertainties are estimated by repeating the analysis with small changes, and taking the uncertainties to be the differences between the values of the various branching ratios before and after the changes. The estimated uncertainties are listed in Tables 9.1-9.6. The overall systematic uncertainties on the results in Tables 9.7-9.9 are obtained by adding in quadrature the individual systematic uncertainties, except on the branching ratios of DCS decays to $D^{0} \rightarrow K^{+} \pi^{-} \pi^{0}$. For these latter measurements, the systematic uncertainty is taken from the fractional uncertainty on the corresponding branching ratio of CF decays to $D^{0} \rightarrow K^{-} \pi^{+} \pi^{0}$. This is done because the fits to the DCS resonances are not stable when the non-resonant 
signal is fitted with a second-order polynomial, as there are too few events.

The first systematic check is to change the non-resonant signal from a linear model to second-order-polynomial model. This allows a significant change in the relative yields of the two signal categories, as the non-resonant portion is free to absorb or reject many events. Because we know that both the non-resonant and resonant portions of the signal are modeled poorly, this is the dominant systematic uncertainty in all the measurements.

The second systematic check is to allow the resonant-signal lineshape to have a mass and width that are not fixed to the RPP values, but are instead allowed to float to values chosen by the fit. This change should not affect the DCS-to-CF branching ratios, since we are still comparing like resonances with identical signal shapes. This assertion was indeed verified. However, as predicted, this check has a significant affect on the branching ratios of CF decays to $D^{0} \rightarrow K^{-} \pi^{+} \pi^{0}$, and this bias tends to counteract that from the first systematic check on the non-resonant shape. 
Table 9.1: Systematic checks and uncertainties of the $R_{K^{*} \pi}$ measurement

\begin{tabular}{lccc}
\hline & $R_{K^{*} \pi}$ & $\begin{array}{c}\text { Statistical } \\
\text { uncertainty }\end{array}$ & $\begin{array}{c}\text { Difference } \\
\text { from } \\
\text { primary result }\end{array}$ \\
\hline Primary result & $1.03 \%$ & $0.06 \%$ & \\
$2^{\text {nd }- \text { order polynomial non-resonant }}$ & $1.08 \%$ & $0.06 \%$ & $+0.05 \%$ \\
\hline
\end{tabular}

Table 9.2: Systematic checks and uncertainties of the $R_{K^{* 0} \pi^{0}}$ measurement

\begin{tabular}{lccc}
\hline & $R_{K^{* 0} \pi^{0}}$ & $\begin{array}{c}\text { Statistical } \\
\text { uncertainty }\end{array}$ & $\begin{array}{c}\text { Difference } \\
\text { from } \\
\text { primary result }\end{array}$ \\
\hline Primary result & $0.22 \%$ & $0.05 \%$ & \\
\begin{tabular}{l}
$2^{\text {nd }}$-order polynomial non-resonant \\
\hline
\end{tabular} & $0.23 \%$ & $0.05 \%$ & $+0.01 \%$ \\
\hline
\end{tabular}

Table 9.3: Systematic checks and uncertainties of the $R_{K \rho}$ measurement

\begin{tabular}{lccc}
\hline & & $\begin{array}{c}\text { Statistical } \\
\text { uncertainty }\end{array}$ & $\begin{array}{c}\text { Difference } \\
\text { from } \\
\text { primary result }\end{array}$ \\
\hline Primary result & $0.102 \%$ & $0.014 \%$ & \\
$2_{K \rho}^{\text {nd }}$-order polynomial non-resonant & $0.117 \%$ & $0.009 \%$ & $+0.015 \%$ \\
\hline
\end{tabular}


Table 9.4: Systematic checks and uncertainties of the $\mathcal{B} r\left(D^{0} \rightarrow K^{*-} \pi^{+}\right)$ measurement

\begin{tabular}{|c|c|c|c|c|}
\hline & & $\mathcal{B} r\left(K^{*-} \pi^{+}\right)$ & $\begin{array}{l}\text { Statistical } \\
\text { uncertainty }\end{array}$ & $\begin{array}{c}\text { Difference } \\
\text { from } \\
\text { primary result }\end{array}$ \\
\hline Primary result & & $9.69 \%$ & $0.09 \%$ & \\
\hline $\begin{array}{l}2^{\text {nd }} \text {-order polynomial } \\
\text { resonant }\end{array}$ & non- & $9.21 \%$ & $0.09 \%$ & $-0.48 \%$ \\
\hline Signal shape floating & & $10.07 \%$ & $0.15 \%$ & $+0.38 \%$ \\
\hline
\end{tabular}

Table 9.5: Systematic checks and uncertainties of the $\mathcal{B} r\left(D^{0} \rightarrow \bar{K}^{* 0} \pi^{0}\right)$ measurement

\begin{tabular}{|c|c|c|c|c|}
\hline & & $\mathcal{B} r\left(\bar{K}^{* 0} \pi^{0}\right)$ & $\begin{array}{l}\text { Statistical } \\
\text { uncertainty }\end{array}$ & $\begin{array}{c}\text { Difference } \\
\text { from } \\
\text { primary result }\end{array}$ \\
\hline Primary result & & $8.88 \%$ & $0.09 \%$ & \\
\hline $\begin{array}{l}2^{\text {nd }} \text {-order polynomial } \\
\text { resonant }\end{array}$ & non- & $8.69 \%$ & $0.09 \%$ & $-0.19 \%$ \\
\hline Signal shape floating & & $9.10 \%$ & $0.11 \%$ & $+0.22 \%$ \\
\hline
\end{tabular}


Table 9.6: Systematic checks and uncertainties of the $\mathcal{B} r\left(D^{0} \rightarrow K^{-} \rho^{+}\right)$measurement

\begin{tabular}{|c|c|c|c|c|}
\hline & & $\mathcal{B} r\left(K^{-} \rho^{+}\right)$ & $\begin{array}{c}\text { Statistical } \\
\text { uncertainty }\end{array}$ & $\begin{array}{c}\text { Difference } \\
\text { from } \\
\text { primary result }\end{array}$ \\
\hline Primary result & & $75.44 \%$ & $0.27 \%$ & \\
\hline $\begin{array}{l}2^{\text {nd }} \text {-order polynomial } \\
\text { resonant }\end{array}$ & non- & $77.28 \%$ & $0.19 \%$ & $+1.84 \%$ \\
\hline Signal shape floating & & $74.54 \%$ & $0.19 \%$ & $-0.9 \%$ \\
\hline
\end{tabular}



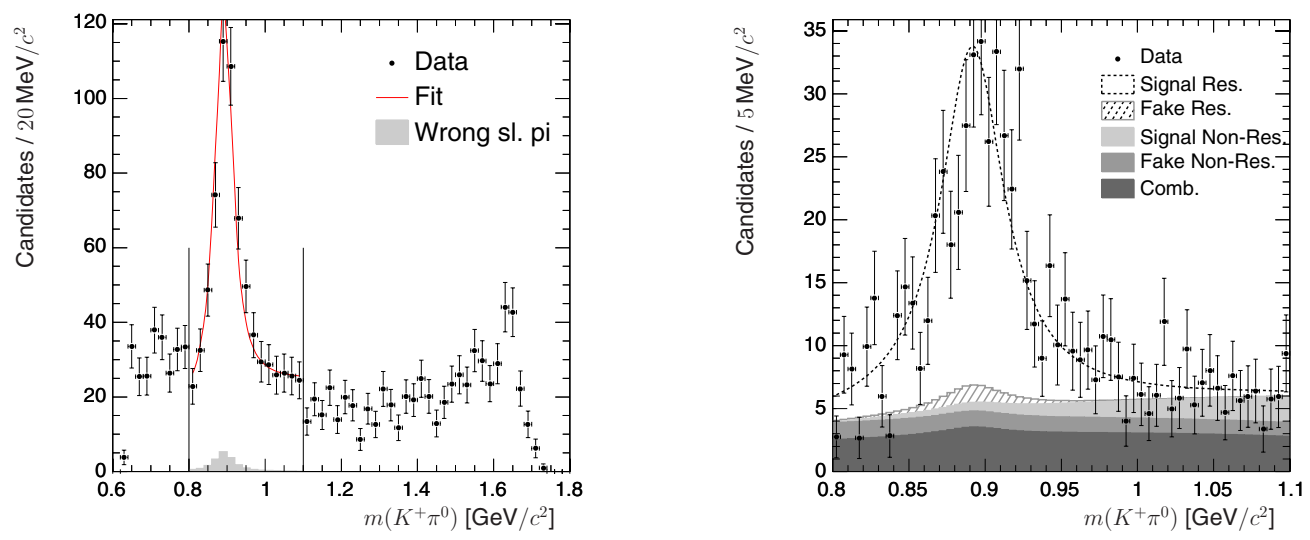

Figure 9.5: Projections of the maximum likelihood fit to the DCS $D^{0} \rightarrow K^{+} \pi^{-} \pi^{0}$ candidates onto $m\left(K^{+} \pi^{0}\right)$, showing the entire kinematic range (left) and the fit region only (right). These projections require $1.85<m_{K \pi \pi^{0}}<1.88 \mathrm{GeV} / c^{2}$ and $0.1444<$ $\Delta m<0.1464 \mathrm{GeV} / c^{2}$. The vertical lines in the left histogram mark the boundaries of the fit region.
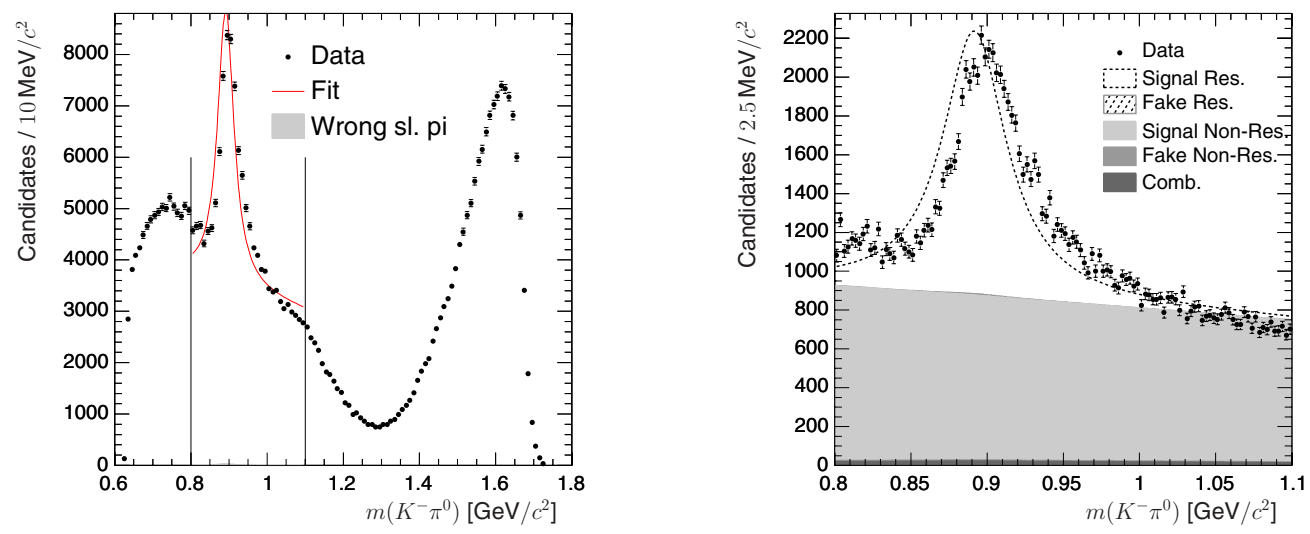

Figure 9.6: Projections of the maximum likelihood fit to the CF $D^{0} \rightarrow K^{-} \pi^{+} \pi^{0}$ candidates onto $m\left(K^{-} \pi^{0}\right)$, showing the entire kinematic range (left) and the fit region only (right). These projections require $1.85<m_{K \pi \pi^{0}}<1.88 \mathrm{GeV} / c^{2}$ and $0.1444<\Delta m<0.1464 \mathrm{GeV} / c^{2}$. The vertical lines in the left histogram mark the boundaries of the fit region. 

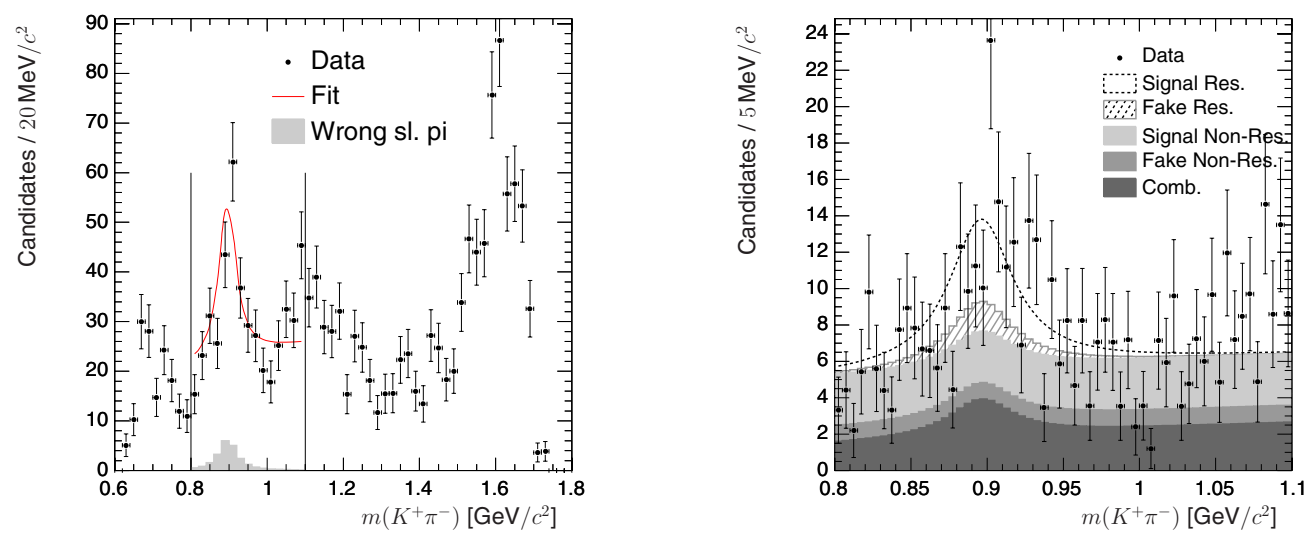

Figure 9.7: Projections of the maximum likelihood fit to the DCS $D^{0} \rightarrow K^{+} \pi^{-} \pi^{0}$ candidates onto $m\left(K^{+} \pi^{-}\right)$, showing the entire kinematic range (left) and the fit region only (right). These projections require $1.85<m_{K \pi \pi^{0}}<1.88 \mathrm{GeV} / c^{2}$ and $0.1444<$ $\Delta m<0.1464 \mathrm{GeV} / c^{2}$. The vertical lines in the left histogram mark the boundaries of the fit region.
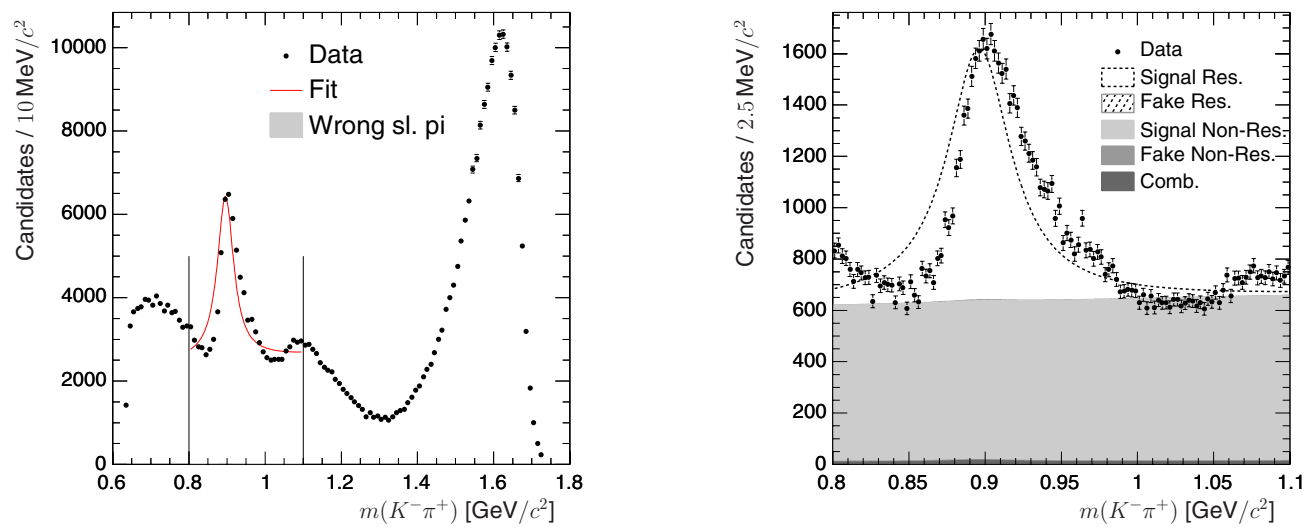

Figure 9.8: Projections of the maximum likelihood fit to the CF $D^{0} \rightarrow K^{-} \pi^{+} \pi^{0}$ candidates onto $m\left(K^{-} \pi^{+}\right)$, showing the entire kinematic range (left) and the fit region only (right). These projections require $1.85<m_{K \pi \pi^{0}}<1.88 \mathrm{GeV} / c^{2}$ and $0.1444<\Delta m<0.1464 \mathrm{GeV} / c^{2}$. The vertical lines in the left histogram mark the boundaries of the fit region. 

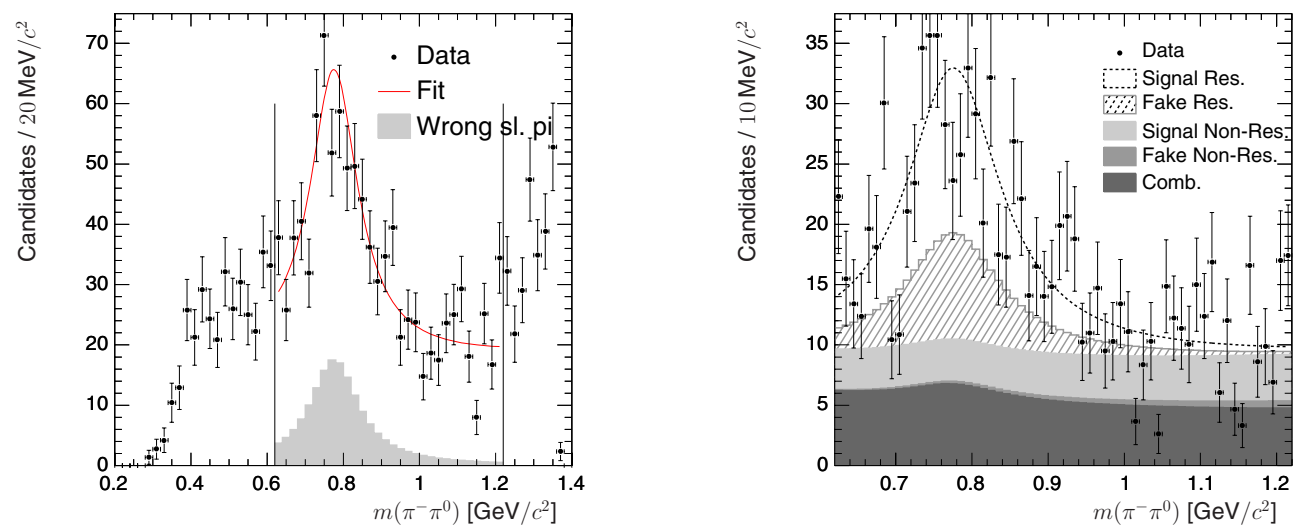

Figure 9.9: Projections of the maximum likelihood fit to the DCS $D^{0} \rightarrow K^{+} \pi^{-} \pi^{0}$ candidates onto $m\left(\pi^{-} \pi^{0}\right)$, showing the entire kinematic range (left) and the fit region only (right). These projections require $1.85<m_{K \pi \pi^{0}}<1.88 \mathrm{GeV} / c^{2}$ and $0.1444<$ $\Delta m<0.1464 \mathrm{GeV} / c^{2}$. The vertical lines in the left histogram mark the boundaries of the fit region.
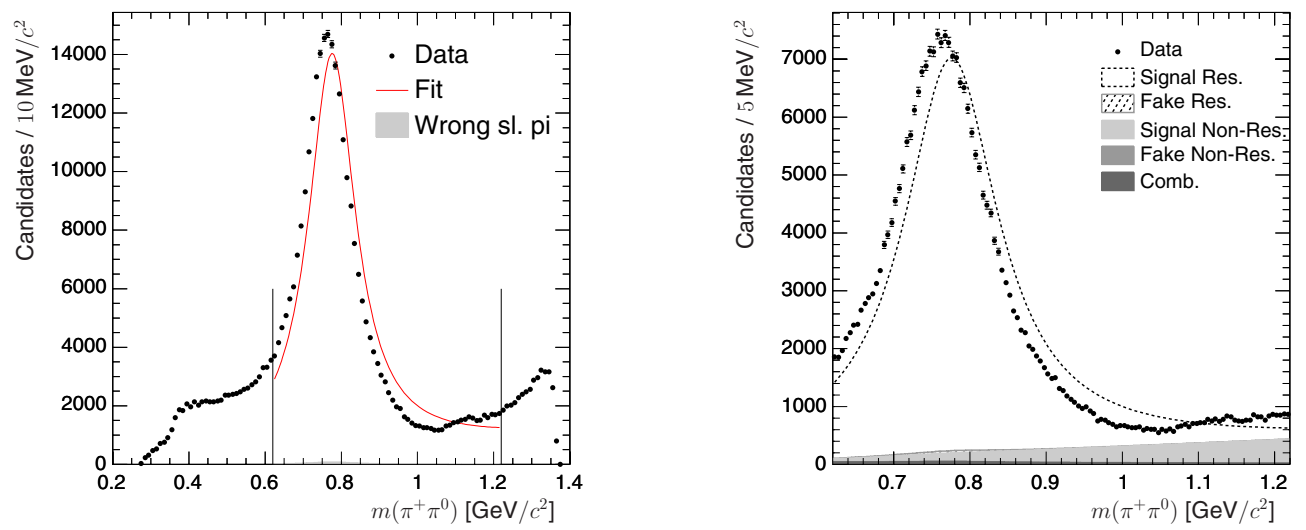

Figure 9.10: Projections of the maximum likelihood fit to the $\mathrm{CF} D^{0} \rightarrow K^{-} \pi^{+} \pi^{0}$ candidates onto $m\left(\pi^{+} \pi^{0}\right)$, showing the entire kinematic range (left) and the fit region only (right). These projections require $1.85<m_{K \pi \pi^{0}}<1.88 \mathrm{GeV} / c^{2}$ and $0.1444<$ $\Delta m<0.1464 \mathrm{GeV} / c^{2}$. The vertical lines in the left histogram mark the boundaries of the fit region. 


\subsection{Final Resonance-Contribution Results}

The final branching ratio measurements are listed in Tables 9.7-9.9. The resonance contributions are indeed very different for $D^{0} \rightarrow K^{+} \pi^{-} \pi^{0}$ decays and $D^{0} \rightarrow K^{-} \pi^{+} \pi^{0}$ decays. For branching ratios involving the DCS decays, the systematic uncertainties are at the same scale as the statistical uncertainties. For the branching ratios involving only $\mathrm{CF}$ decays, the systematic uncertainties are much larger than the statistical uncertainties, which is expected because of the lineshapes used. However, the $\mathcal{B} r\left(D^{0} \rightarrow K^{*-} \pi^{+}\right)$and $\mathcal{B} r\left(D^{0} \rightarrow \bar{K}^{* 0} \pi^{0}\right)$ results are much lower than the values listed in the RPP, and it is possible that the results presented here may in fact be more accurate despite the relatively large systematic uncertainties involved. A Dalitzplot analysis of the CF decays in this data set seems well-motivated based on these results. 
Table 9.7: Branching ratios of doubly Cabibbo-suppressed decays to corresponding Cabibbo-favored decays.

$$
\begin{array}{lr}
\Gamma\left(D^{0} \rightarrow K^{*+} \pi^{-}\right) / \Gamma\left(D^{0} \rightarrow K^{*-} \pi^{+}\right) & (1.03 \pm 0.06(\text { stat }) \pm 0.05(\text { syst })) \% \\
\Gamma\left(D^{0} \rightarrow K^{* 0} \pi^{0}\right) / \Gamma\left(D^{0} \rightarrow \bar{K}^{* 0} \pi^{0}\right) & (0.22 \pm 0.05(\text { stat }) \pm 0.01(\text { syst })) \% \\
\Gamma\left(D^{0} \rightarrow K^{+} \rho^{-}\right) / \Gamma\left(D^{0} \rightarrow K^{-} \rho^{+}\right) & (0.102 \pm 0.014(\text { stat }) \pm 0.015(\text { syst })) \%
\end{array}
$$

Table 9.8: Branching ratios of doubly Cabibbo-suppressed decays to $D^{0} \rightarrow K^{+} \pi^{-} \pi^{0}$

\begin{tabular}{crr}
\hline$D^{0}$ Decay Mode & Events & $\Gamma_{i} / \Gamma\left(D^{0} \rightarrow K^{+} \pi^{-} \pi^{0}\right)$ \\
\hline$D^{0} \rightarrow K^{*+} \pi^{-}$ & $(5.37 \pm 0.32) \times 10^{2}$ & $(46.1 \pm 3.3($ stat $) \pm 2.9($ syst $)) \%$ \\
& $K^{*+} \rightarrow K^{+} \pi^{0}$ & \\
$D^{0} \rightarrow K^{* 0} \pi^{0}$ & $(1.05 \pm 0.26) \times 10^{2}$ & $(9.0 \pm 2.3($ stat $) \pm 0.3($ syst $)) \%$ \\
$K^{* 0} \rightarrow K^{+} \pi^{-}$ & $(4.0 \pm 0.5) \times 10^{2}$ & $(36 \pm 5($ stat $) \pm 1($ syst $)) \%$ \\
$D^{0} \rightarrow K^{+} \rho^{-}$ & & \\
\hline
\end{tabular}

Table 9.9: Branching ratios of Cabibbo-favored decays to $D^{0} \rightarrow K^{-} \pi^{+} \pi^{0}$

\begin{tabular}{ccc}
\hline$D^{0}$ Decay Mode & Events & $\Gamma_{i} / \Gamma\left(D^{0} \rightarrow K^{-} \pi^{+} \pi^{0}\right)$ \\
\hline$D^{0} \rightarrow K^{*-} \pi^{+}$ & $(5.22 \pm 0.05) \times 10^{4}$ & $(9.7 \pm 0.1$ (stat $) \pm 0.6($ syst $)) \%$ \\
$K^{*-} \rightarrow K^{-} \pi^{0}$ & & \\
$D^{0} \rightarrow \bar{K}^{* 0} \pi^{0}$ & $(4.78 \pm 0.05) \times 10^{4}$ & $(8.88 \pm 0.09($ stat $) \pm 0.29($ syst $)) \%$ \\
$\bar{K}^{* 0} \rightarrow K^{-} \pi^{+}$ & & \\
$D^{0} \rightarrow K^{-} \rho^{+}$ & $(3.901 \pm 0.012) \times 10^{5}$ & $(75.4 \pm 0.3($ stat $) \pm 2.1$ (syst $)) \%$ \\
\hline
\end{tabular}




\section{Chapter 10}

\section{Analysis of the $D^{0}$ Decay-Time}

\section{Distribution}

\subsection{Introduction}

In order to search for $D^{0}-\bar{D}^{0}$ mixing, a maximum likelihood fit to the $D^{0}$ decaytime distribution ${ }^{1}$ must be performed. This includes fitting both the right-sign $D^{0} \rightarrow K^{-} \pi^{+} \pi^{0}$ and the wrong-sign $D^{0} \rightarrow K^{+} \pi^{-} \pi^{0}$ decay-time distributions. The likelihood fit is a three-dimensional fit to $\left\{m_{K \pi \pi^{0}}, \Delta m, t_{K \pi \pi^{0}}\right\}$ with the likelihood defined by Equation 6.1. The two dimensions of $\left\{m_{K \pi \pi^{0}}, \Delta m\right\}$ are fit to the same probability density functions (PDFs) as in Chapter 6, and they are needed to accurately

${ }^{1}$ Charge conjugation is implied except where otherwise stated. 
separate signal from the three background categories. The third observable $t_{K \pi \pi^{0}}$ is fit to different signal PDFs for the right-sign and wrong-sign decays, respectively, but the two types of decays have the same background PDFs. As in the measurement of the branching ratio, the high-statistics sample of right-sign decays serves to determine precisely the exponential form of the doubly Cabibbo-suppressed decay-time distribution, and it is in the deviation from this well-determined form that one may observe $D$ mixing in the wrong-sign sample. We begin by fitting the right-sign decay-time distribution, and proceed to analyze and fit the wrong-sign decay-time distribution to search for $D$ mixing.

\subsection{Measured Decay-Time Uncertainties}

As described in Section 4.3, the entire $D^{*+} \rightarrow D^{0} \pi_{s}^{+}, D^{0} \rightarrow K^{\mp} \pi^{ \pm} \pi^{0}$ decay chain is fit at once in the final candidate selection, and from the simultaneous fit of both the $D^{*+}$ and $D^{0}$ decay vertices, the $D^{0}$ decay time, $t_{K \pi \pi^{0}}$, and its associated uncertainty, $\sigma_{t}$, are calculated. The decay-time is

$$
t_{K \pi \pi^{0}}=\left(\frac{m_{D^{0}}}{p}\right) \Delta x
$$

where $p$ is the $D^{0}$ momentum and $\Delta x$ is the distance between the $D^{*+}$ and $D^{0}$ decay vertices. The uncertainty is calculated using the decay-fit covariance matrix, and it includes the correlations between the two vertex positions. In fitting to the decay-time 

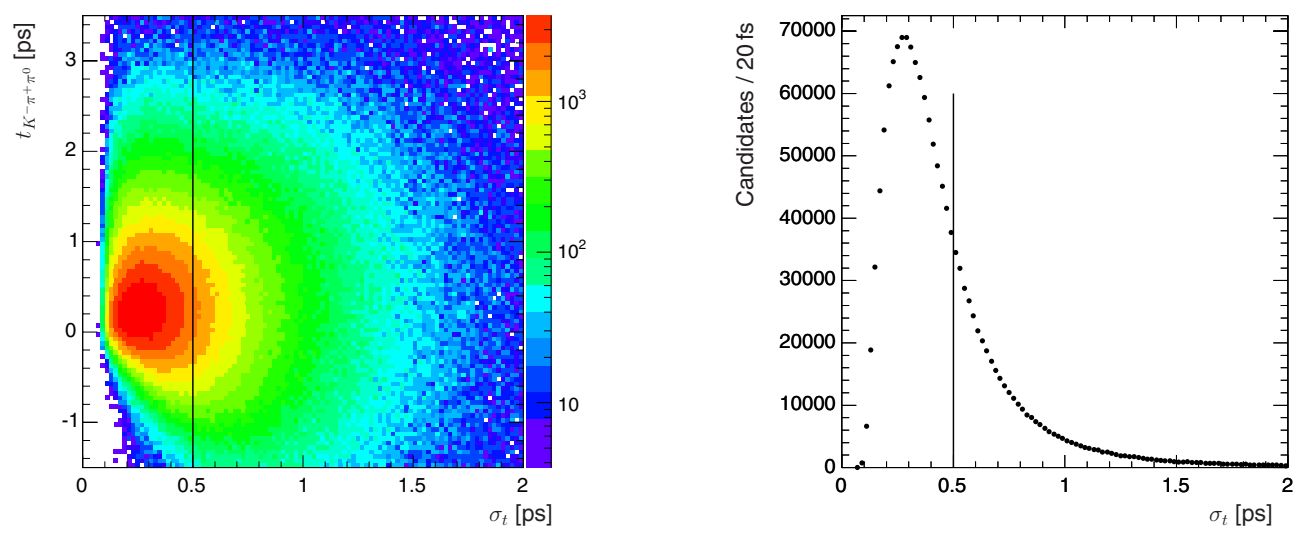

Figure 10.1: Measured $D^{0}$ decay-time-uncertainty distributions for $1.74<m_{K \pi \pi^{0}}<$ $1.98 \mathrm{GeV} / c^{2}$ and $0.13957<\Delta m<0.155 \mathrm{GeV} / c^{2}$. The solid vertical line in both histograms shows the selection requirement on $\sigma_{t}$ for events to be fitted. The mean value of $\sigma_{t}$ for signal events is near $0.43 \mathrm{fs}$.

distribution, we exclude candidates with poorly measured decay times by requiring

$$
\sigma_{t}<0.5 \mathrm{ps}
$$

Figure 10.1 illustrates both the relationship between $\sigma_{t}$ and $t_{K \pi \pi^{0}}$ and the overall $\sigma_{t}$ distribution. For signal events, the mean $\sigma_{t}$ is near 0.43 ps. Although we do not fit a PDF to the $\sigma_{t}$ distribution, the per-event values are used in the decay-time distribution fit. 


\subsection{Description of Right-Sign Probability Density}

\section{Functions}

\subsubsection{Signal}

The signal PDF for the decay time is based on an exponential shape, convolved with a Gaussian resolution function to account for the uncertainty in the decay-time measurement:

$$
\begin{aligned}
\rho_{R S, i}(t ; \tau, k) & =e^{-t / \tau} \otimes \exp \left(-\frac{t^{2}}{2\left(k \sigma_{i}\right)^{2}}\right) \\
& =\exp \left(-\frac{t}{\tau}+\frac{\left(k \sigma_{i}\right)^{2}}{2 \tau^{2}}\right) \operatorname{erfc}\left(-\frac{t}{\sqrt{2}\left(k \sigma_{i}\right)}+\frac{k \sigma_{i}}{\sqrt{2} \tau}\right)
\end{aligned}
$$

In this PDF, the index $i$ spans the events, representing the fact that the uncertainty $\sigma_{i}$ is measured for each event as described in Section 10.2. The resolution model is specified by the parameter $k$, which is a scale factor $\approx \mathcal{O}(1)$ by which the event uncertainty is multiplied. The assumption underlying this choice of resolution model is that the uncertainties are relatively accurate, but they may be systematically underor over-estimated by a global scale factor. This choice of resolution model has proven to work well in previous analyses.

The complete, three-dimensional signal PDF is

$$
\begin{aligned}
\mathcal{P}_{\mathrm{RS}, i}(m, \Delta m, t) & = \\
\mathcal{S}(m, \Delta m) & \times\left\{f_{t 3} \cdot \rho_{3, i}+\left(1-f_{t 3}\right) \cdot\left[f_{t 2} \cdot \rho_{2, i}+\left(1-f_{t 2}\right) \cdot \rho_{1, i}\right]\right\}
\end{aligned}
$$




$$
\begin{aligned}
& \rho_{1, i}=\rho_{R S, i}\left(t ; \tau, k_{1}\right) \\
& \rho_{2, i}=\rho_{R S, i}\left(t ; \tau, k_{2}\right) \\
& \rho_{3, i}=\rho_{R S, i}\left(t ; \tau, k_{3}\right)
\end{aligned}
$$

where $\mathcal{S}(m, \Delta m)$ is defined in Equation 6.6 and $\left\{f_{t 3}, f_{t 2}\right\}$ are parameters representing the fraction of events described by the various Gaussian resolution functions.

\subsubsection{Combinatoric Background}

This background, described in Section 6.2.2, does not have a lifetime associated with it because it comprises tracks that do not come from any definite decay. One might expect it to be represented by a Gaussian centered at $t=0$; in fact, it is slightly biased towards positive decay times, and it has a tail in the positive direction. The tail in the distribution is fit using a Crystal Ball function similar to Equation 6.4 but with the power-law of the attenuation allowed to vary:

$$
\begin{gathered}
c b(x ; \bar{x}, \sigma, \alpha, n)= \begin{cases}\exp \left(-\frac{(x-\bar{x})^{2}}{2 \sigma^{2}}\right) & \text { if } \frac{(x-\bar{x})}{\sigma}<\alpha \\
a\left(b+\frac{(x-\bar{x})}{\sigma}\right)^{-n} & \text { if } \frac{(x-\bar{x})}{\sigma} \geq \alpha\end{cases} \\
\alpha>0, \quad a=(1 / \alpha) \exp \left(\alpha^{2} / 2\right), \quad b=(1 / \alpha)-\alpha
\end{gathered}
$$


The complete three-dimensional PDF for this background category is

$$
\begin{aligned}
& \mathcal{P}_{\text {comb }}(m, \Delta m, t)= \\
& \mathcal{C}(m, \Delta m) \times\left\{f_{c 2} \cdot g\left(t ; \bar{t}_{2}, \sigma_{c 2}\right)+\left(1-f_{c 2}\right) \cdot c b\left(t ; \bar{t}_{1}, \sigma_{c 1}, \alpha_{c}, n_{c}\right)\right\}
\end{aligned}
$$

where $\mathcal{C}(m, \Delta m)$ is defined in Equation 6.8, $g\left(t ; \bar{t}_{2}, \sigma_{c 2}\right)$ is a Gaussian, and $f_{c 2}$ is a parameter representing the fraction of events described by the Gaussian.

\subsubsection{Bad- $D^{*+}$ Background}

This background, described in Section 6.2 .3 , is due to real $D^{0}$ decays with uncorrelated $\pi_{s}^{ \pm}$candidates. Because the $D^{*+}$ decay vertex is primarily determined by the intersection of the $D^{0}$ with the beamspot, and the $D^{0}$ candidate is real, this background has the same shape as the signal, with the same lifetime. Thus, the decay-time PDF is given by Equation 10.4, and we have

$$
\begin{aligned}
& \mathcal{P}_{\text {fake }, i}(m, \Delta m, t)= \\
& \mathcal{F}(m, \Delta m) \times\left\{f_{t 3} \cdot \rho_{3, i}+\left(1-f_{t 3}\right) \cdot\left[f_{t 2} \cdot \rho_{2, i}+\left(1-f_{t 2}\right) \cdot \rho_{1, i}\right]\right\}
\end{aligned}
$$

where $\mathcal{F}(m, \Delta m)$ is defined in Equation 6.9.

\subsubsection{Bad- $D^{0}$ Background}

This background, described in Section 6.2.4, is due to particles produced by a $D^{*+}$ decay that has been reconstructed incorrectly. These particles may have a measurable 
lifetime that is different from the signal distribution. The decay-time PDF used to describe this category is the same as Equation 10.4 but with a different lifetime, $\tau_{B}$; the full PDF is

$$
\begin{aligned}
\mathcal{P}_{\text {brok }, i}(m, \Delta m, t)= & \mathcal{B}(m, \Delta m) \times\left\{f_{t 3} \cdot \rho_{3 B, i}\right. \\
& \left.+\left(1-f_{t 3}\right) \cdot\left[f_{t 2} \cdot \rho_{2 B, i}+\left(1-f_{t 2}\right) \cdot \rho_{1 B, i}\right]\right\} \\
& \rho_{1 B, i}=\rho_{R S, i}\left(t ; \tau_{B}, k_{1}\right) \\
& \rho_{2 B, i}=\rho_{R S, i}\left(t ; \tau_{B}, k_{2}\right) \\
& \rho_{3 B, i}=\rho_{R S, i}\left(t ; \tau_{B}, k_{3}\right)
\end{aligned}
$$

where $\mathcal{B}(m, \Delta m)$ is defined in Equation 6.11.

\subsection{Right-Sign Fit Results}

\section{and $D^{0}$ Lifetime Measurement}

The unbinned maximum likelihood fit described above is performed in two steps.

First, the two-dimensional $\left\{m_{K \pi \pi^{0}}, \Delta m\right\}$ fit is performed to adjust all the parameters and yields to the data set with the requirement $\sigma_{t}<0.5 \mathrm{ps}$. The signal yield after performing this step is $7.307 \times 10^{5} \pm 1.2 \times 10^{3}$. Second, the parameters of the twodimensional fit are fixed, and the three-dimensional fit is performed to find the best values of the parameters in the decay-time PDFs. The $\left\{m_{K \pi \pi^{0}}, \Delta m\right\}$ PDF parameters 
are fixed because the decay-time distribution is not capable of separating the various categories better than the mass distributions. The final fit is shown projected onto the $t_{K \pi \pi^{0}}$ axis for various regions of $\left\{m_{K \pi \pi^{0}}, \Delta m\right\}$ in Figures 10.2-10.6.

The scale factors $\left\{k_{1}, k_{2}, k_{3}\right\}$ are initially allowed to float in the fit, and their final values are given in Table 10.1. The values of $\left\{k_{1}, k_{2}\right\}$, which are the scale factors for the resolution functions that together account for over $95 \%$ of the events, are very close to one, indicating that the measurement uncertainties are for the most part very accurate. The third scale factor, $k_{3}$, is larger and accounts for events with severely mismeasured vertices. After an initial minimization, these values are fixed because they are highly correlated with the parameters representing the fraction of events described by each resolution function.

Table 10.1: Decay-time-uncertainty scale factors from the likelihood fit

$$
\begin{array}{ll}
k_{1}: & 1.060 \\
k_{2}: & 0.925 \\
k_{3}: & 1.950
\end{array}
$$

The fitted lifetime is

$$
\tau=0.4127 \pm 0.0006 \text { fs (stat) }
$$

where the systematic uncertainty is omitted.

Without evaluating all of the systematic uncertainties relating to this lifetime measurement, we would like to assess how accurate it is. To do this, we measure the 
lifetime in a complementary way that does not require the fit to the decay-time distribution, and we compare the two values. Another way to measure the lifetime is to calculate the simple mean of the decay-time distribution, taking advantage of the fact that

$$
\left\langle e^{-t / \tau}\right\rangle=\tau
$$

As long as the resolution of the decay-time measurements is not skewed, the mean of the measured decay-time distribution will be $\tau$.

Using the full dataset fitted with the two-dimensional PDF described in Chapter 6, each event is assigned the signal weight defined in Equation 8.3. The lifetime is then calculated as

$$
\tau=\frac{\sum_{i} w_{\text {sig }, i} t_{i}}{\sum_{i} w_{\text {sig }, i}}
$$

where $i$ spans all of the events. Evaluating the lifetime in this manner yields

$$
\tau=0.4092 \pm 0.0004 \mathrm{fs}(\text { stat })
$$

Both of these lifetime measurements are expected to have uncertainties that are dominated by systematic effects. Comparing these values with each other and with the accepted lifetime of

$$
\tau_{\mathrm{RPP}}=0.4103 \pm 0.0015 \mathrm{fs}
$$

reported in the Review of Particle Physics [23], we believe that the lifetime fit and the data are sufficiently well understood to proceed with an analysis of the wrong-sign 

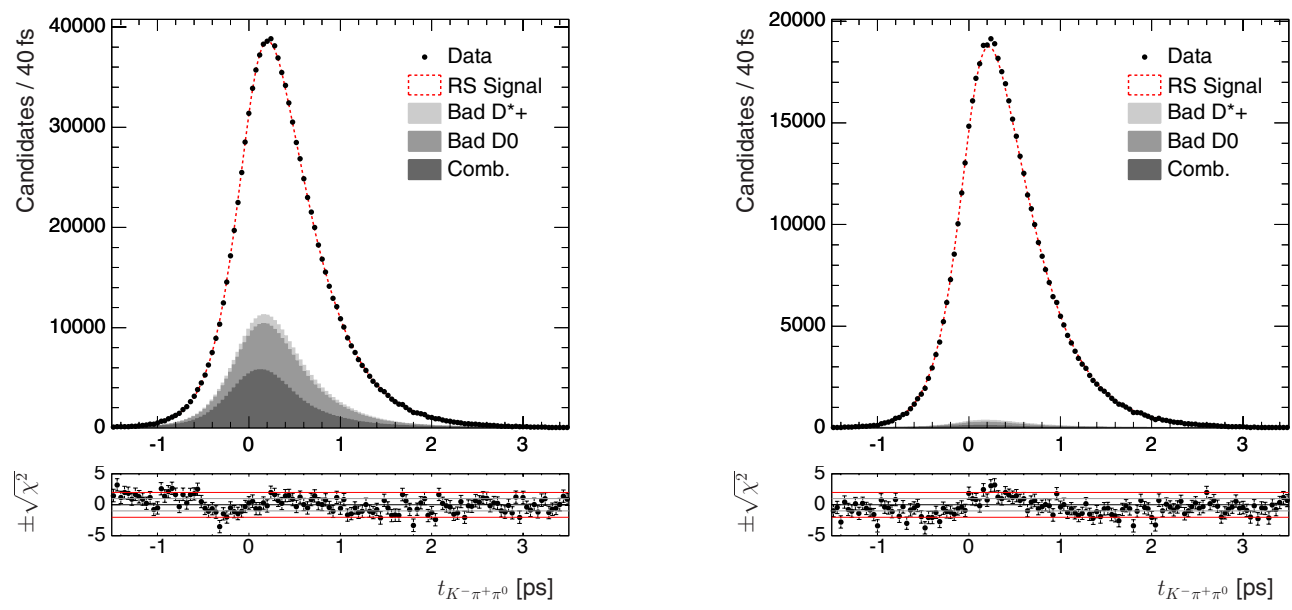

Figure 10.2: Projections of the maximum likelihood fit to the right-sign data onto $t_{K \pi \pi^{0}}$ for the entire fit region (left) and for the signal region only (right). The entire fit region is $1.74<m_{K \pi \pi^{0}}<1.98 \mathrm{GeV} / c^{2}$ and $0.13957<\Delta m<0.155 \mathrm{GeV} / c^{2}$; the signal region is $1.85<m_{K \pi \pi^{0}}<1.88 \mathrm{GeV} / c^{2}$ and $0.1444<\Delta m<0.1464 \mathrm{GeV} / c^{2}$.

decay-time distribution.

\subsection{Selection of Dalitz-Plot Regions}

Using the knowledge of the doubly Cabibbo-suppressed resonance contributions gained from the analysis in Chapter 9, we seek to maximize sensitivity to a signal of $D$ mixing by performing the lifetime analysis in a region of the Dalitz plot that minimizes $R_{I}$, where $R_{I}$ is the integrated value of $R_{D}$ over the selected Dalitz-plot region. We choose to do this by excluding regions of the Dalitz plot that are primarily 

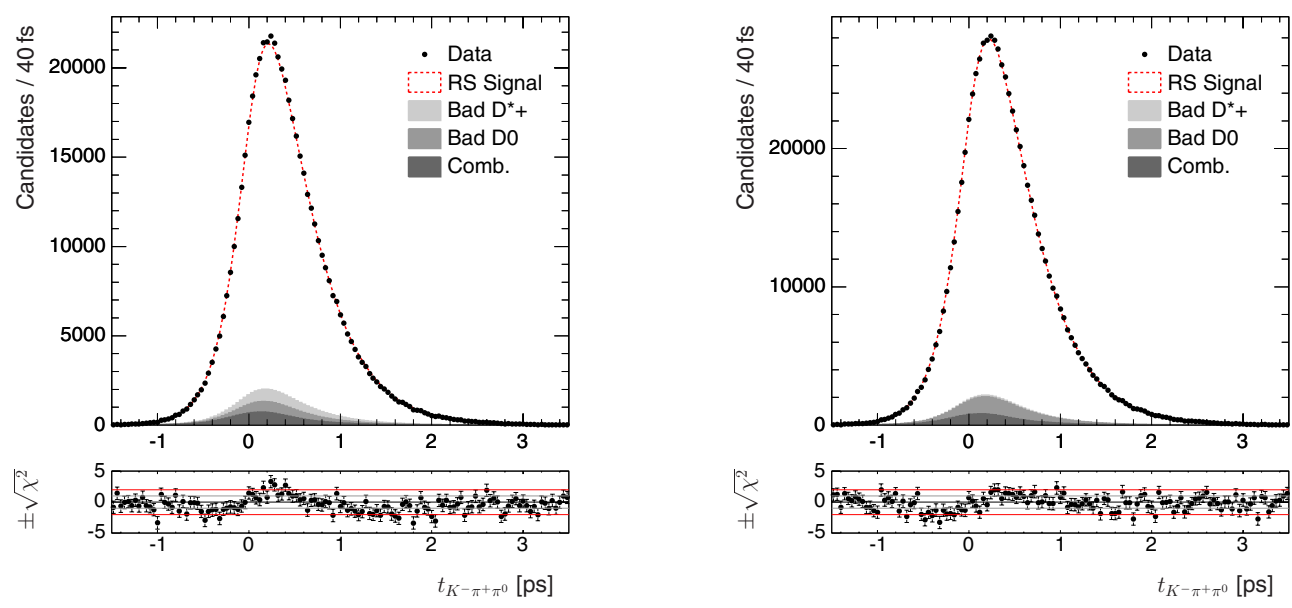

Figure 10.3: Projections of the maximum likelihood fit to the right-sign data onto $t_{K \pi \pi^{0}}$ for the $m_{K \pi \pi^{0}}$ signal region (left) and for the $\Delta m$ signal region (right). The $m_{K \pi \pi^{0}}$ signal region is $1.85<m_{K \pi \pi^{0}}<1.88 \mathrm{GeV} / c^{2}$ and $0.13957<\Delta m<$ $0.155 \mathrm{GeV} / c^{2}$; the $\Delta m$ signal region is $1.74<m_{K \pi \pi^{0}}<1.98 \mathrm{GeV} / c^{2}$ and $0.1444<$ $\Delta m<0.1464 \mathrm{GeV} / c^{2}$.
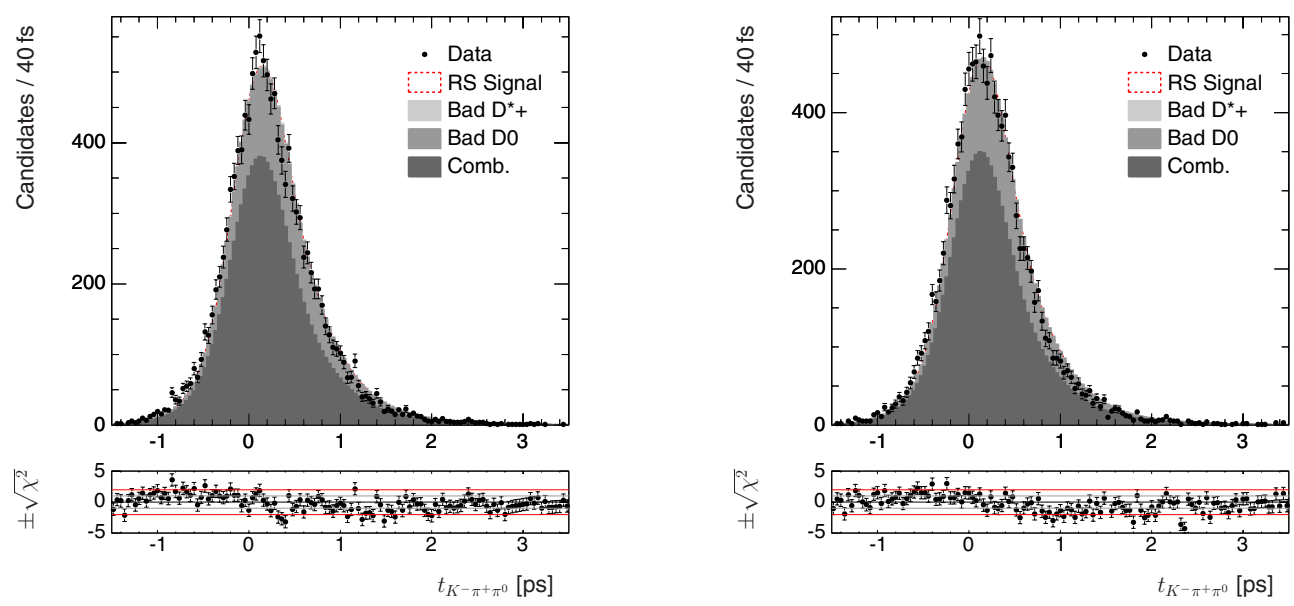

Figure 10.4: Projections of the maximum likelihood fit to the right-sign data onto $t_{K \pi \pi^{0}}$ for sideband regions emphasizing the combinatoric background in the lower $m_{K \pi \pi^{0}}$ region (left) and in the upper $m_{K \pi \pi^{0}}$ region (right). The lower $m_{K \pi \pi^{0}}$ region is $1.74<m_{K \pi \pi^{0}}<1.82 \mathrm{GeV} / c^{2}$ and $0.152<\Delta m<0.155 \mathrm{GeV} / c^{2}$; the upper $m_{K \pi \pi^{0}}$ region is $1.91<m_{K \pi \pi^{0}}<1.98 \mathrm{GeV} / c^{2}$ and $0.152<\Delta m<0.155 \mathrm{GeV} / c^{2}$. 


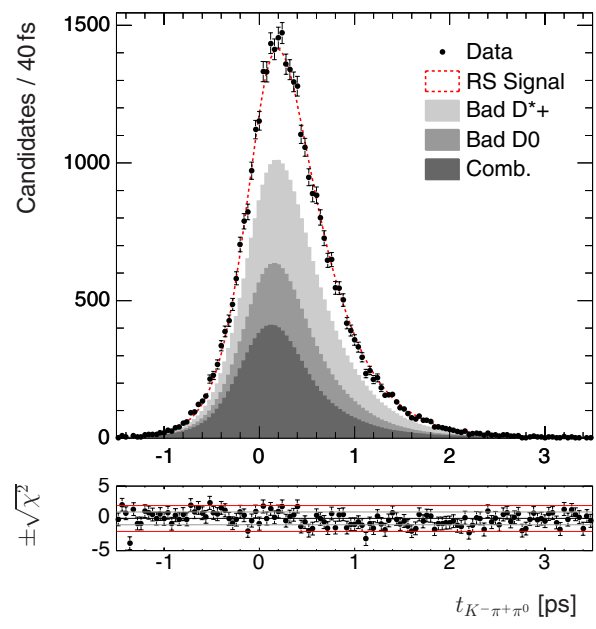

Figure 10.5: Projections of the maximum likelihood fit to the right-sign data onto $t_{K \pi \pi^{0}}$ for a sideband region emphasizing the bad- $D^{*+}$ background. The region is $1.85<m_{K \pi \pi^{0}}<1.88 \mathrm{GeV} / c^{2}$ and $0.147<\Delta m<0.155 \mathrm{GeV} / c^{2}$.
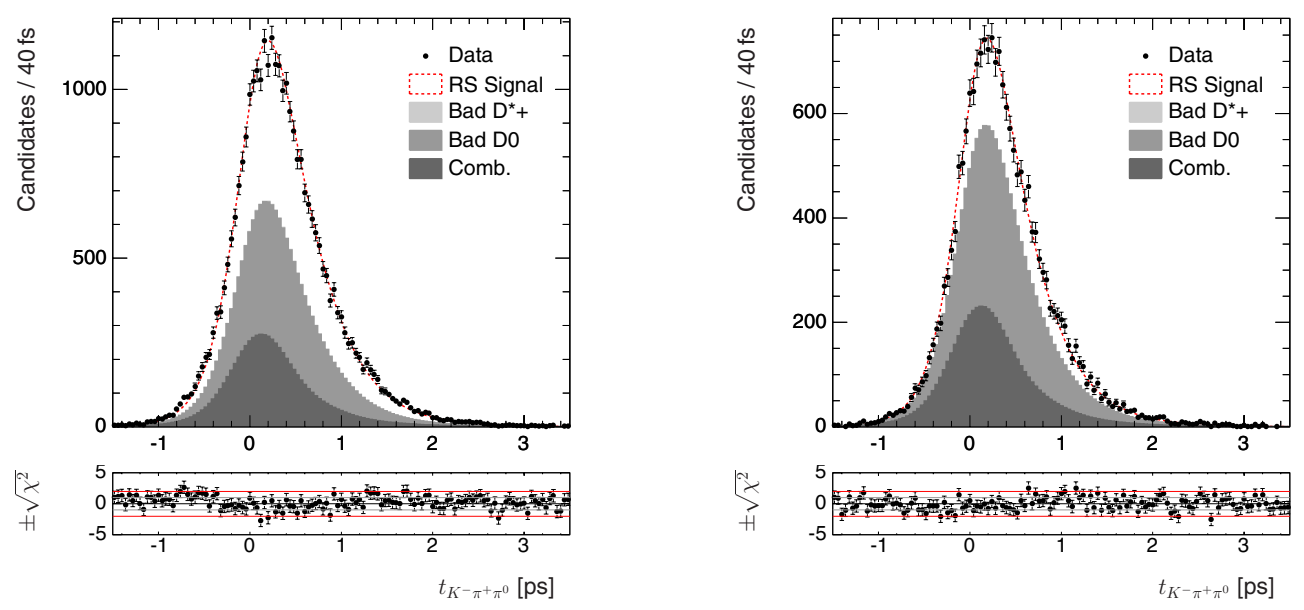

Figure 10.6: Projections of the maximum likelihood fit to the right-sign data onto $t_{K \pi \pi^{0}}$ for sideband regions emphasizing the bad- $D^{0}$ background in the lower $m_{K \pi \pi^{0}}$ region (left) and in the upper $m_{K \pi \pi^{0}}$ region (right). The lower $m_{K \pi \pi^{0}}$ region is $1.74<$ $m_{K \pi \pi^{0}}<1.82 \mathrm{GeV} / c^{2}$ and $0.1444<\Delta m<0.1464 \mathrm{GeV} / c^{2}$; the upper $m_{K \pi \pi^{0}}$ region is $1.91<m_{K \pi \pi^{0}}<1.98 \mathrm{GeV} / c^{2}$ and $0.1444<\Delta m<0.1464 \mathrm{GeV} / c^{2}$. 
populated by the $K^{*}$ and $K^{* 0}$ resonances. Specifically, we select events such that

$$
\begin{gathered}
m(K \pi)<850 \mathrm{MeV} / c^{2} \text { or } m(K \pi)>950 \mathrm{MeV} / c^{2} \\
m\left(K \pi^{0}\right)<850 \mathrm{MeV} / c^{2} \text { or } m\left(K \pi^{0}\right)>950 \mathrm{MeV} / c^{2}
\end{gathered}
$$

Performing the two-dimensional $\left\{m_{K \pi \pi^{0}}, \Delta m\right\}$ maximum likelihood fit on this data set (including the requirement $\sigma_{t}<0.5 \mathrm{ps}$ ) yields

$$
R_{I}=(0.150 \pm 0.011) \%
$$

with the fitted number of wrong-sign signal events equal to $(7.6 \pm 0.5) \times 10^{2}$. This defines the final selection of data to be included in the $D$-mixing decay-time fit.

\subsection{Description of Wrong-Sign Signal Probability Density Function}

\subsubsection{Motivation and Definition of a Functional Form}

Recall from Section 2.3 the time-dependent decay rate including $D$ mixing for a multi-body decay (Equation 2.32):

$$
\begin{gathered}
\Gamma_{\bar{f}}(t)=\bar{A}_{I}^{2} e^{-\Gamma t}\left(R_{I}+\alpha \sqrt{R_{I}} y_{I}^{\prime}(\Gamma t)+\frac{x^{2}+y^{2}}{4}(\Gamma t)^{2}\right), \\
0 \leq \alpha \leq 1
\end{gathered}
$$


Previous analyses of the $D^{0} \rightarrow K^{+} \pi^{-}$lifetime have fitted for $\left\{x^{\prime 2}, y^{\prime}\right\}$, where $\left\{x^{\prime}, y^{\prime}\right\}$ are the quantities $\{x, y\}$ rotated by a strong phase shift $\delta$,

$$
\begin{aligned}
& x^{\prime} \equiv x \cos \delta+y \sin \delta \\
& y^{\prime} \equiv y \cos \delta-x \sin \delta,
\end{aligned}
$$

and

$$
\left(x^{\prime 2}+y^{\prime 2}\right)=\left(x^{2}+y^{2}\right) .
$$

Because the multi-body decay rate has an additional factor $\alpha$, to which we have no direct sensitivity, we cannot directly fit the form given by Equation 2.32. Also, in general, fitting for $\left\{x^{\prime 2}, y^{\prime}\right\}$ is not preferable for two reasons. First, there is a strong correlation between these two parameters in the likelihood fit, making it difficult for the fit to converge reliably. Second, the likelihood function in terms of $\left\{x^{\prime 2}, y^{\prime}\right\}$ is non-parabolic near the likelihood maximum, so the fit returns inaccurate uncertainty estimates. For all of these reasons, a different functional form is used for the maximum likelihood fit to the wrong-sign decay-time distribution.

Consider the function

$$
\Gamma(t) \propto e^{-\Gamma t}\left(a_{1}^{2}+\left(\frac{a_{1} a_{3}}{1+a_{2}^{2}}\right)(\Gamma t)+\left(\frac{a_{3}^{2}}{4}\right)(\Gamma t)^{2}\right)
$$


We can relate physically meaningful quantities to the parameters $\left\{a_{1}, a_{2}, a_{3}\right\}$ :

$$
\begin{aligned}
R_{I} & =a_{1}^{2} \\
\alpha y_{I}^{\prime} & =\frac{a_{3}}{1+a_{2}^{2}} \\
\left(x^{2}+y^{2}\right) / 2 & =a_{3}^{2} / 2
\end{aligned}
$$

In this form, the amplitude of the mixing rate, $\left(x^{2}+y^{2}\right) / 2$, is determined by $a_{3}$; the sign of the interference term is also determined by $a_{3}$; and the size of the interference term is determined by $a_{2}$. The sign of $a_{1}$ is stable because the value of $R_{I}$ is welldetermined and not near zero. The mixing rate has the property that it is always nonnegative, which makes the fit much more stable. However, because the sign of $a_{3}$ is relevant to determining the likelihood value, $a_{3}$ is not significantly biased towards a non-zero value. Because $a_{3}$ is present in the interference term, and the observation of the interference term is necessarily an observation of $D$ mixing, this form increases sensitivity to $D$ mixing.

The disadvantage to this form is that the parameter $a_{2}$ is not well-defined for fitting purposes. There may be a cusp in the likelihood function at $a_{2}=0$ because the sign of $a_{2}$ is irrelevant in determining the likelihood. Also, as $a_{2} \rightarrow \infty$, the likelihood becomes insensitive to the precise value of $a_{2}$, making estimation of its uncertainty impossible. However, these considerations do not inhibit for a $D$-mixing search, because neither $\alpha$ nor $y_{I}^{\prime}$ have predicted values for either the Dalitz plot as a whole or for any region of the Dalitz plot. The specific size of the interference term is not a 
physically important quantity — the interference term is treated here as a tool for gaining sensitivity to the mixing term, $\left(x^{2}+y^{2}\right)$. It should be noted, however, that even though the uncertainty estimation for $a_{2}$ may not be accurate, the physical quantity $\alpha y_{I}^{\prime}$ will always have sensible values.

The signal PDF for the wrong-sign decay time is based on Equation 10.20, convolved with a Gaussian resolution function:

$$
\begin{aligned}
& \rho_{W S, i}\left(t ; \tau, a_{1}, a_{2}, a_{3}, k\right)= \\
& e^{-t / \tau}\left(a_{1}^{2}+\left(\frac{a_{1} a_{3}}{1+a_{2}^{2}}\right)\left(\frac{t}{\tau}\right)+\left(\frac{a_{3}^{2}}{4}\right)\left(\frac{t}{\tau}\right)^{2}\right) \\
& \quad \otimes \exp \left(-\frac{t^{2}}{2\left(k \sigma_{i}\right)^{2}}\right) .
\end{aligned}
$$

The complete, three-dimensional wrong-sign signal PDF is

$$
\begin{gathered}
\mathcal{P}_{\mathrm{WS}, i}(m, \Delta m, t)= \\
\mathcal{S}(m, \Delta m) \times\left\{f_{t 3} \cdot \rho_{3, i}^{\prime}+\left(1-f_{t 3}\right) \cdot\left[f_{t 2} \cdot \rho_{2, i}^{\prime}+\left(1-f_{t 2}\right) \cdot \rho_{1, i}^{\prime}\right]\right\}(10.25) \\
\rho_{1, i}^{\prime}=\rho_{\mathrm{WS}, i}\left(t ; \tau, a_{1}, a_{2}, a_{3}, k_{1}\right) \\
\rho_{2, i}^{\prime}=\rho_{\mathrm{WS}, i}\left(t ; \tau, a_{1}, a_{2}, a_{3}, k_{2}\right) \\
\rho_{3, i}^{\prime}=\rho_{\mathrm{WS}, i}\left(t ; \tau, a_{1}, a_{2}, a_{3}, k_{3}\right)
\end{gathered}
$$

where $\mathcal{S}(m, \Delta m)$ is defined in Equation 6.6 and $\left\{f_{t 3}, f_{t 2}\right\}$ are parameters representing the fraction of events described by the various Gaussian resolution functions. 


\subsubsection{Validation of the Functional Form}

In order to verify that the functional form defined in Equation 10.24 can be fit to the data reliably, and that the parameter values at the likelihood maximum are not significantly biased, a number of Monte Carlo data sets are generated and the signal PDF is fit to them. Seven ensembles of 1000 data sets are generated and fit, and the parameters used to generate the ensembles are listed in Table 10.2. Each data set is generated with a 1000 events. The generating parameters are chosen such that one of the ensembles has no mixing, and the remaining six are split between those with a negative interference term and those with a positive interference term. Because these tests are simply to determine whether or not the fit of this PDF has any intrinsic bias, the samples are generated without any background. Another critical feature of these tests is that $a_{1}$ is fixed in the fits, while $\left\{a_{2}, a_{3}\right\}$ are allowed to vary. This is done because, for these tests, an extended maximum likelihood fit is not performed-the number of events in the sample is not allowed to vary-and therefore $a_{1}$ would not be adequately constrained.

The figures of merit for these tests are the pull distributions of $a_{3}$, in which we verify that there is limited bias in the fitted values and that the statistical uncertainties are well-estimated. We do not expect $a_{2}$ to have well-behaved estimated uncertainties, so we ignore its pull distributions. This parameter may be regarded as a necessary degree of freedom provided to the fitter, the specific value of which is sufficiently 
Table 10.2: Parameters used for generating wrong-sign Monte Carlo events.

\begin{tabular}{rrr}
\hline$R_{I}$ & $\alpha y_{I}^{\prime}$ & $x^{2}+y^{2}$ \\
\hline 0.0015 & 0.0 & 0.0 \\
& & \\
0.0015 & -0.01166 & 0.000136 \\
0.0015 & -0.00583 & 0.000136 \\
0.0015 & -0.00117 & 0.000136 \\
& & \\
0.0015 & 0.01166 & 0.000136 \\
0.0015 & 0.00583 & 0.000136 \\
0.0015 & 0.00117 & 0.000136 \\
\hline
\end{tabular}

decoupled from the mixing term.

Histograms of the pull distributions are shown in Figures 10.7-10.10. For the case of no $D$ mixing (Figure 10.7), the distribution is centered at zero with no significant bias. For the case of mixing with a large interference term (Figure 10.8), the distributions are nearly Gaussian and minimally biased. For the case of mixing with smaller interference terms (Figure 10.9,10.10), the distributions become non-Gaussian and biased because it is more difficult for the fit to correctly determine the sign of $a_{3}$. However, they are biased such that the fits tend toward a smaller level of $D$ mixing than was generated.

Finally, contours of $\Delta \log \mathcal{L}$ are shown in the $\left\{a_{2}, a_{3}\right\}$ plane for three individual Monte Carlo event sets in Figures 10.11-10.13. These contours give an indication of how sensitive we might be to a $D$-mixing signal, what the statistical uncertainties might be, and the size of any potential bias resulting from fitting this particular func- 
tion to the data. Using a Bayesian interpretation of the likelihood contours, we may calculate the expected values and variances of $a_{3}$ and the related physical quantity $\left(x^{2}+y^{2}\right) / 2$, according to the formulas

$$
\begin{aligned}
E[x] & =\int x e^{\log \mathcal{L}\left(a_{2}, a_{3}\right)} d a_{2} d a_{3} \\
& =\int x \mathcal{L}\left(a_{2}, a_{3}\right) d a_{2} d a_{3} \\
\sigma_{x}^{2} & =\int(x-E(x))^{2} \mathcal{L}\left(a_{2}, a_{3}\right) d a_{2} d a_{3},
\end{aligned}
$$

where $x$ can be either $a_{3}= \pm \sqrt{x^{2}+y^{2}}$ or $\left(x^{2}+y^{2}\right) / 2$. For each of Figures 10.1110.13 , the expected value of $a_{3}, E\left[a_{3}\right]$, is represented by a dotted line and the $1 \sigma$ region $\pm \sigma_{a_{3}}$ by hatches.

In all of the three cases studied, the generated value of $a_{3}$ is included in the $1 \sigma$ region calculated according to Equation 10.27. In general, given the conditions of approximately 1000 wrong-sign events with $R_{I} \approx 0.15 \%$ and no background, it appears we may expect uncertainties of $\sigma_{a_{3}} \approx 0.004$ and $\sigma_{\left(x^{2}+y^{2}\right) / 2} \approx(0.004) \%$. For comparison, the 95\%-confidence limit set by the BABAR Collaboration [10] using $D^{0} \rightarrow K^{+} \pi^{-}$decays is $\left(x^{2}+y^{2}\right) / 2<0.13 \%$. 


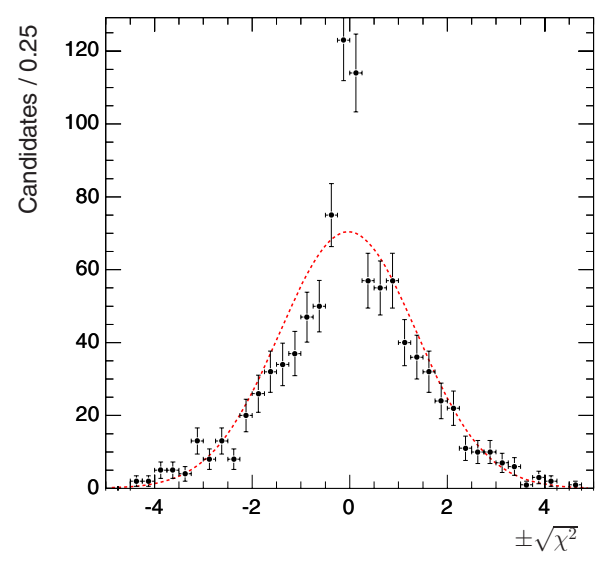

Figure 10.7: Pull distributions $\left( \pm \sqrt{\chi^{2}}\right)$ for the parameter $a_{3}$ after fitting 1000 Monte Carlo event sets generated with no mixing. The distribution is from sets generated with $\left\{\alpha y_{I}^{\prime},\left(x^{2}+y^{2}\right)\right\}=\{0.0,0.0\}$. The distribution mean is $-0.029 \pm 0.045$, and the width is $1.404 \pm 0.032$.
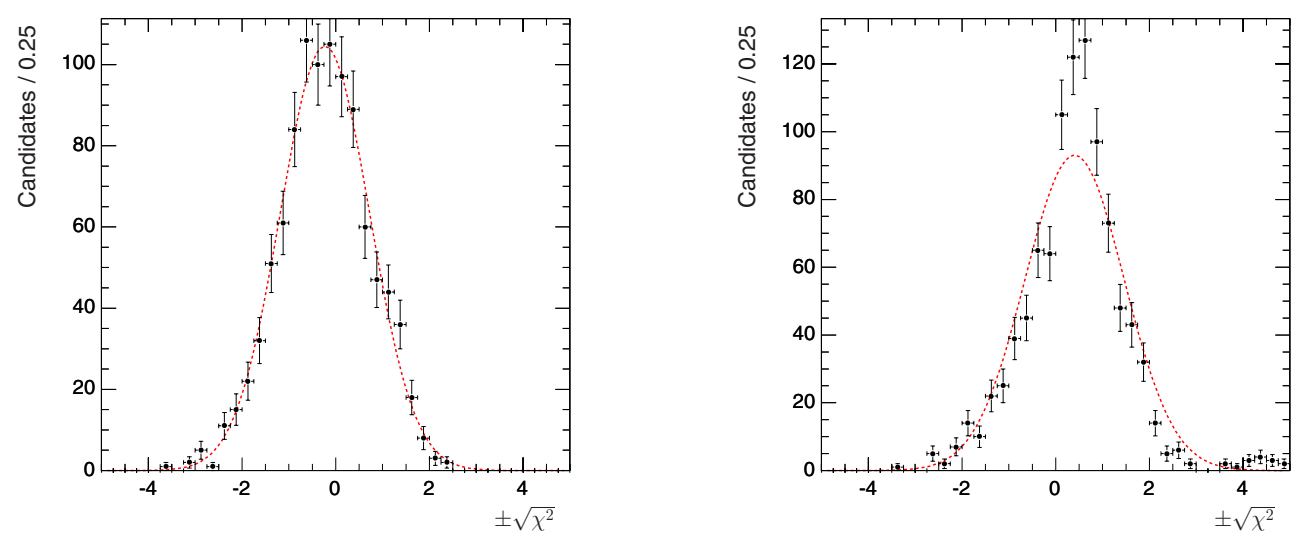

Figure 10.8: Pull distributions $\left( \pm \sqrt{\chi^{2}}\right)$ for the parameter $a_{3}$ after fitting 1000 Monte Carlo event sets generated with a large interference term. The distribution on the left is from sets generated with $\left\{\alpha y_{I}^{\prime},\left(x^{2}+y^{2}\right)\right\}=\{-0.01166,0.000136\}$. The distribution mean is $-0.231 \pm 0.030$, and the width is $0.952 \pm 0.021$. The distribution on the right is from sets generated with $\left\{\alpha y_{I}^{\prime},\left(x^{2}+y^{2}\right)\right\}=\{0.01166,0.000136\}$. The distribution mean is $0.403 \pm 0.034$, and the width is $1.057 \pm 0.024$. 

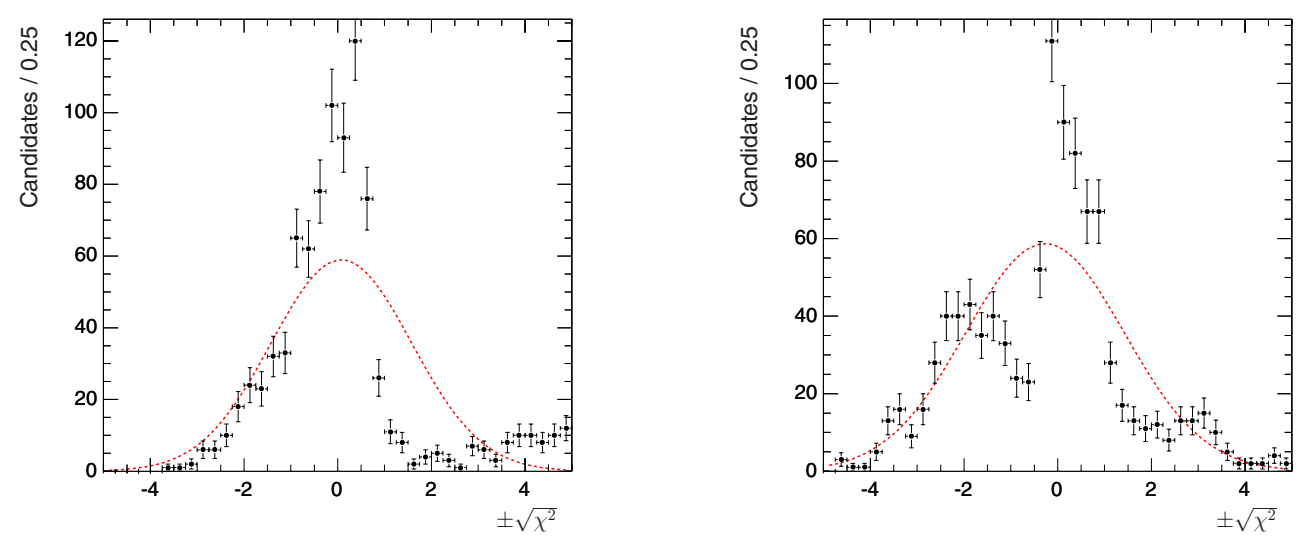

Figure 10.9: Pull distributions $\left( \pm \sqrt{\chi^{2}}\right)$ for the parameter $a_{3}$ after fitting 1000 Monte Carlo event sets generated with a moderate interference term. The distribution on the left is from sets generated with $\left\{\alpha y_{I}^{\prime},\left(x^{2}+y^{2}\right)\right\}=\{-0.00583,0.000136\}$. The distribution mean is $0.079 \pm 0.051$, and the width is $1.500 \pm 0.037$. The distribution on the right is from sets generated with $\left\{\alpha y_{I}^{\prime},\left(x^{2}+y^{2}\right)\right\}=\{0.00583,0.000136\}$. The distribution mean is $-0.271 \pm 0.055$, and the width is $1.698 \pm 0.041$.
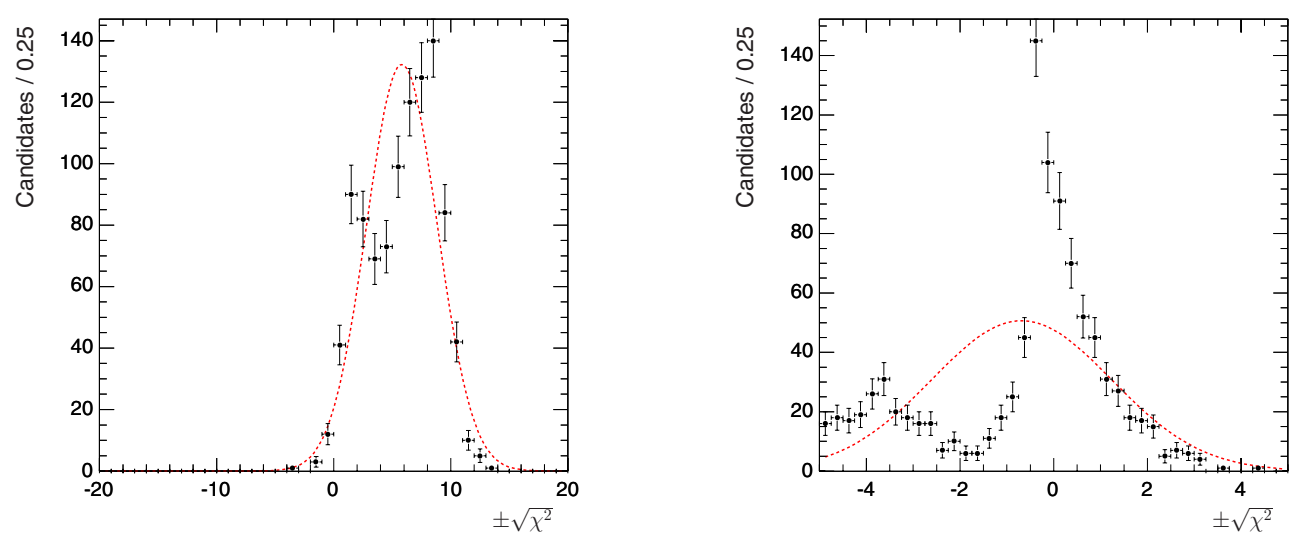

Figure 10.10: Pull distributions $\left( \pm \sqrt{\chi^{2}}\right)$ for the parameter $a_{3}$ after fitting 1000 Monte Carlo event sets generated with a small interference term. The distribution on the left is from sets generated with $\left\{\alpha y_{I}^{\prime},\left(x^{2}+y^{2}\right)\right\}=\{-0.00117,0.000136\}$. The distribution mean is $5.823 \pm 0.0 .95$, and the width is $3.001 \pm 0.067$. The distribution on the right is from sets generated with $\left\{\alpha y_{I}^{\prime},\left(x^{2}+y^{2}\right)\right\}=\{0.00117,0.000136\}$. The distribution mean is $-0.687 \pm 0.065$, and the width is $1.923 \pm 0.053$. 

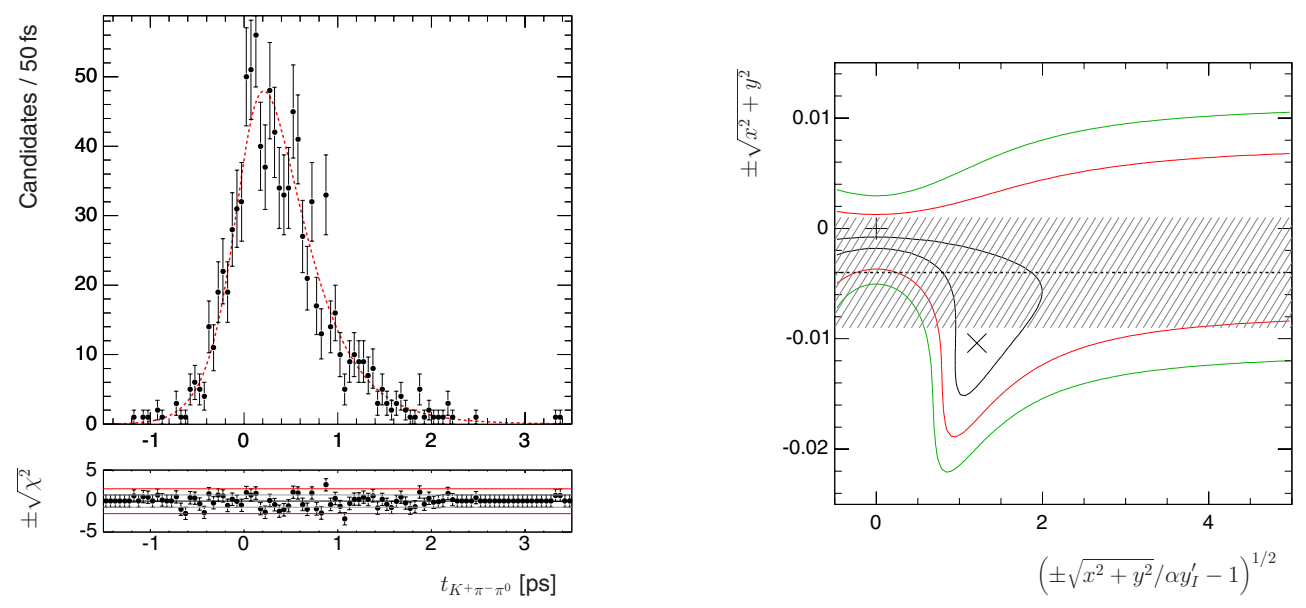

Figure 10.11: Fit results for a fit to a wrong-sign Monte Carlo event set with 1000 events, generated with no mixing. On the left is the decay-time distribution with the fit overlaid. On the right are contours of $\Delta \log \mathcal{L}=0.5,2.0,4.5$, representing $1 \sigma, 2 \sigma$, and $3 \sigma$ contours in black, red, and green, respectively. The plus (+) marks the generated parameter values, and the cross $(\mathrm{X})$ marks the fitted parameter values. The dotted line marks the expected value of $\pm \sqrt{x^{2}+y^{2}}$, calculated by integrating the likelihood function over $\left\{a_{2}, a_{3}\right\}$, and the hatched region shows the $1 \sigma$ limits. For this generated data set, we find $\pm \sqrt{x^{2}+y^{2}}=-0.004 \pm 0.005$ and $\left(x^{2}+y^{2}\right) / 2=(0.0021 \pm 0.0029) \%$ 

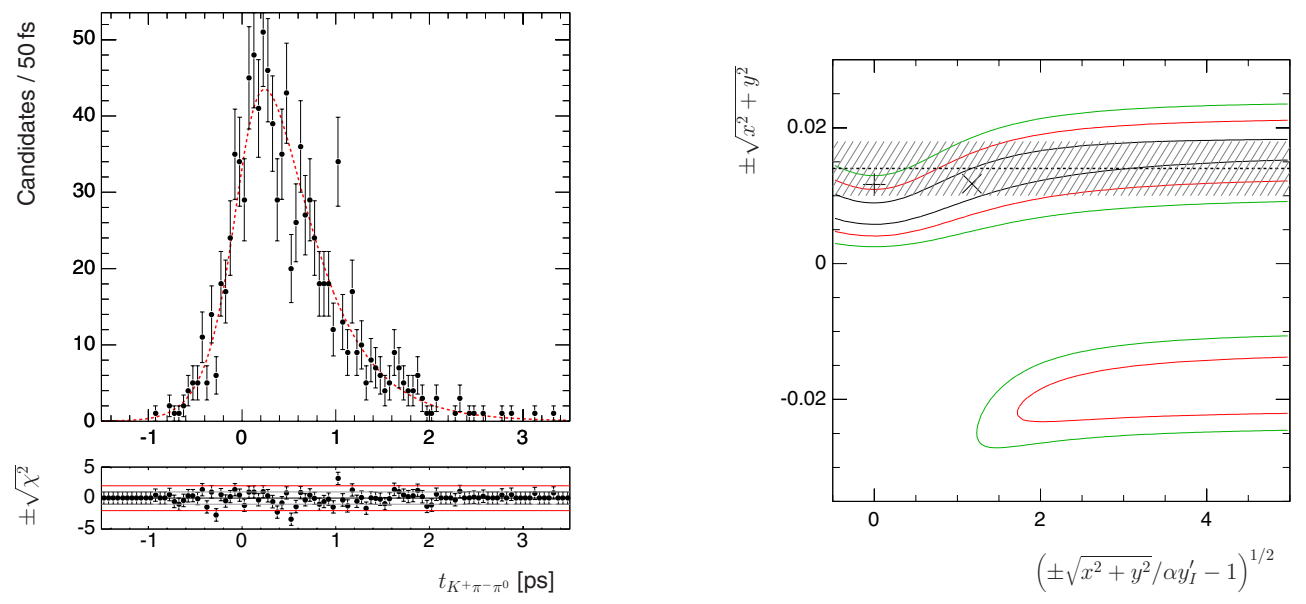

Figure 10.12: Fit results for a fit to a wrong-sign Monte Carlo event set with 1000 events, generated with mixing and a large positive interference term. On the left is the decay-time distribution with the fit overlaid. On the right are contours of $\Delta \log \mathcal{L}=0.5,2.0,4.5$, representing $1 \sigma, 2 \sigma$, and $3 \sigma$ contours in black, red, and green, respectively. The plus (+) marks the generated parameter values, and the cross $(\mathrm{X})$ marks the fitted parameter values. The dotted line represents the expected value of $\pm \sqrt{x^{2}+y^{2}}$, calculated by integrating the likelihood function over $\left\{a_{2}, a_{3}\right\}$, and the hatched region shows the $1 \sigma$ limits. For this generated data set, we find $\pm \sqrt{x^{2}+y^{2}}=0.014 \pm 0.004$ and $\left(x^{2}+y^{2}\right) / 2=(0.012 \pm 0.006) \%$ 

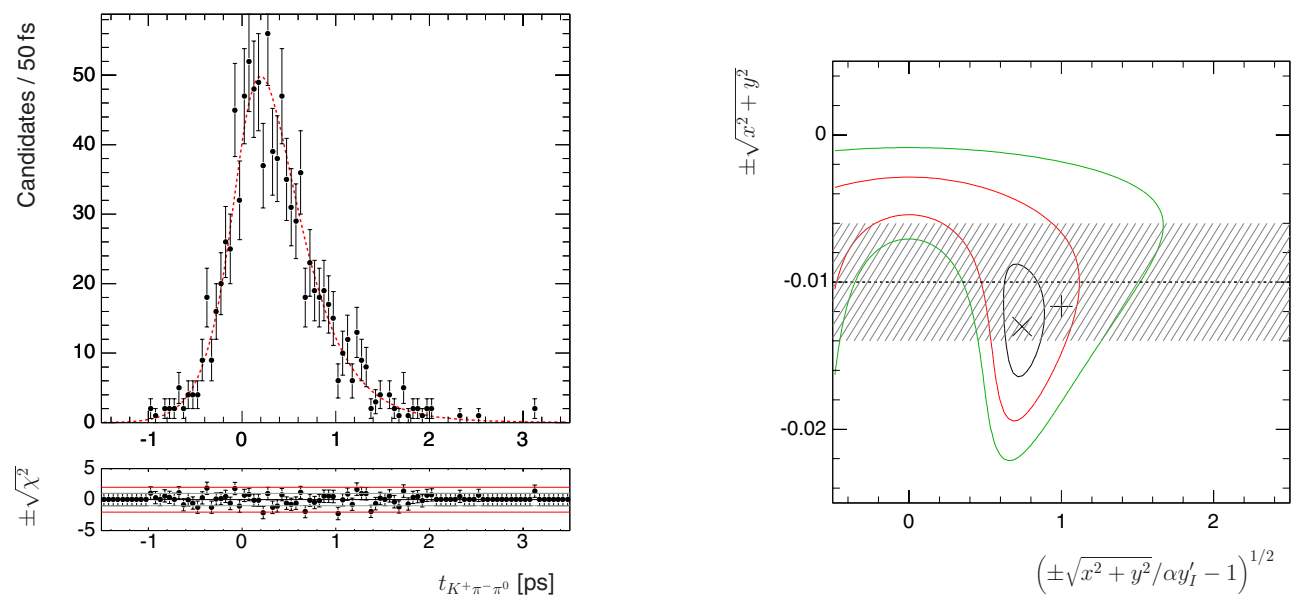

Figure 10.13: Fit results for a fit to a wrong-sign Monte Carlo event set with 1000 events, generated with mixing and a moderate negative interference term. On the left is the decay-time distribution with the fit overlaid. On the right are contours of $\Delta \log \mathcal{L}=0.5,2.0,4.5$, representing $1 \sigma, 2 \sigma$, and $3 \sigma$ contours in black, red, and green, respectively. The plus (+) marks the generated parameter values, and the cross (X) marks the fitted parameter values. The dotted line marks the expected value of $\pm \sqrt{x^{2}+y^{2}}$, calculated by integrating the likelihood function over $\left\{a_{2}, a_{3}\right\}$, and the hatched region shows the $1 \sigma$ limits. For this generated data set, we find $\pm \sqrt{x^{2}+y^{2}}=$ $-0.010 \pm 0.004$ and $\left(x^{2}+y^{2}\right) / 2=(0.006 \pm 0.004) \%$ 


\subsection{Wrong-Sign Fit Results}

\section{and $D$-Mixing Measurement}

The results of the maximum likelihood fit to the right-sign sample are used as fixed inputs to the wrong-sign fit. It is observed that the combinatoric background in the wrong-sign sample has a slightly different shape from that in the right-sign sample, so the relevant parameters are initially allowed to vary in the fit. After the fit has converged with the parameters $\left\{a_{2}, a_{3}\right\}$ fixed, all of the parameters are fixed to their final values except for $\left\{a_{1}, a_{2}, a_{3}\right\}$. Finally, the fit is performed for these three parameters, and the fit is shown to converge. In each of these steps, an extended likelihood fit is performed, so that the value of $a_{1}$ determines the number of doubly Cabibbosuppressed events in the sample, which is very near the total number of fitted signal events. The final parameter values are given in Table 10.3, and the corresponding physical values are given in Table 10.4. The statistical uncertainties given are those returned by the minimization program, and they are not expected to be accurate; they are listed only for completeness.

The final fit is shown projected onto the $t_{K \pi \pi^{0}}$ axis for various regions of $\left\{m_{K \pi \pi^{0}}, \Delta m\right\}$ in Figures 10.15-10.19. The likelihood contours for the final data set are shown in Figure 10.14.

Given the parameter values at the maximal likelihood and the likelihood varia- 
Table 10.3: Fitted values of mixing parameters, corresponding to the likelihood maximum

$$
\begin{array}{lr}
a_{1}: & 0.0431 \pm 0.0029 \\
a_{2}: & 0.88 \pm 0.30 \\
a_{3}: & -0.022 \pm 0.008
\end{array}
$$

Table 10.4: Values of physical quantities, calculated from the fit parameters

$$
\begin{aligned}
R_{I}: & (0.186 \pm 0.025) \% \\
\alpha y^{\prime}: & -0.012 \pm 0.007 \\
\left(x^{2}+y^{2}\right) / 2: & (0.024 \pm 0.017) \%
\end{aligned}
$$

tions as a function of $\left\{a_{1}, a_{2}, a_{3}\right\}$, we now attempt to establish an unbiased result for $\left(x^{2}+y^{2}\right) / 2$ with an accurate uncertainty estimate. The guide in this effort will be the estimated consistency of this data set with a result of no $D$ mixing. This consistency is determined by generating 10,000 Monte Carlo data sets with no mixing, fitting the signal PDF to each of these simulated data sets, and recording a metric indicating whether the simulated data set or the real data set is a better match to the no-mixing, or null, hypothesis. A simulated data set is considered more consistent with the null hypothesis than the real data set if

$$
\begin{aligned}
\left(\frac{\mathcal{L}\left(\vec{x}_{\text {null }}\right)}{\mathcal{L}\left(\vec{x}_{\text {max }}\right)}\right)_{\text {MC }} & >\left(\frac{\mathcal{L}\left(\vec{x}_{\text {null }}\right)}{\mathcal{L}\left(\vec{x}_{\text {max }}\right)}\right)_{\text {Data }} \\
a_{3} & <0
\end{aligned}
$$

where $\mathcal{L}(\vec{x})$ is the likelihood of the indicated data set for the values $\vec{x}=\left\{a_{1}, a_{2}, a_{3}\right\}$, $\vec{x}_{\text {null }}$ represents the parameter values in the null hypothesis, and $\vec{x}_{\max }$ represents the 
Table 10.5: Monte Carlo data sets demonstrating the consistency of the real data set with no mixing

\begin{tabular}{lr}
\hline Relationship to Real Data & Monte Carlo Sets \\
\hline More consistent with no mixing, $a_{3}<0.0$ & 3,986 \\
More consistent with no mixing, $a_{3}>0.0$ & 4,408 \\
Less consistent with no mixing, $a_{3}<0.0$ & 958 \\
Less consistent with no mixing, $a_{3}>0.0$ & 648 \\
\hline
\end{tabular}

parameter values that maximize the likelihood for the indicated data set. The value of $a_{3}$ is required to be less than zero in this test because the fit to the real data set prefers a negative value, and we believe that the fit has sensitivity to the sign of $a_{3}$. The results of this consistency test are listed in Table 10.5. We find that of 10,000 simulated data sets, there are 958 cases in which the maximal likelihood of the simulated data occurs for $a_{3}<0$ and the physical data are more consistent with the null hypothesis than the simulated data. Therefore, we conclude that the real data set is consistent with the null hypothesis at the $10 \%$ confidence level.

Given the above conclusion, we would like to find an expected value of $a_{3}$ with symmetric errors that include $a_{3}=0$ at the $10 \%$, or $1.3-\sigma$, level. Considering the likelihood contours in Figure 10.14, this is equivalent to finding an appropriate region over which to integrate Equations $10.26-10.27$ in the parameter space of $\left\{a_{1}, a_{2}, a_{3}\right\}$. Choosing a region where $0<a_{2}<\infty$ will yield an expected value of $a_{3}$ that is zero. Alternatively, choosing a region where $a_{2}$ does not vary significantly will yield limits that are too narrow. We choose a region in $a_{2}$ that is symmetric about the fitted value 
and that reproduces a no-mixing result at the $10 \%$-confidence level. The integration limits for the calculation of the expected value of $a_{3}$ are shown in Figure 10.14 as vertical lines. The resulting expected value of $a_{3}$, with symmetric $1 \sigma$ intervals, is shown as a horizontal dotted line within a hatched region.

Using the above prescription, we measure the $D$-mixing rate to be

$$
\begin{aligned}
& \pm \sqrt{x^{2}+y^{2}}=-0.013 \pm 0.010 \\
& \left(\frac{x^{2}+y^{2}}{2}\right)=(0.013 \pm 0.013) \%
\end{aligned}
$$

The sign of $\pm \sqrt{x^{2}+y^{2}}$ indicates the sign of the interference term, which appears to be negative for the Dalitz-plot region selected in this analysis.

Systematic uncertainties have not been evaluated for these measurements. However, based on the knowledge of previous $D$ mixing searches, we expect systematic uncertainties in these results to be small compared to the estimated statistical uncertainty.

To help understand the sensitivity of the fit to mixing, the fit is shown projected onto the $t_{K \pi \pi^{0}}$ axis in Figure 10.20 after the parameters $\left\{a_{1}, a_{2}, a_{3}\right\}$ have been adjusted to the null hypothesis. The difference between the fit after it has been adjusted to the null hypothesis and the fit at the likelihood maximum is almost imperceptible; this is expected, since the background contributions in these projections are dominant. 


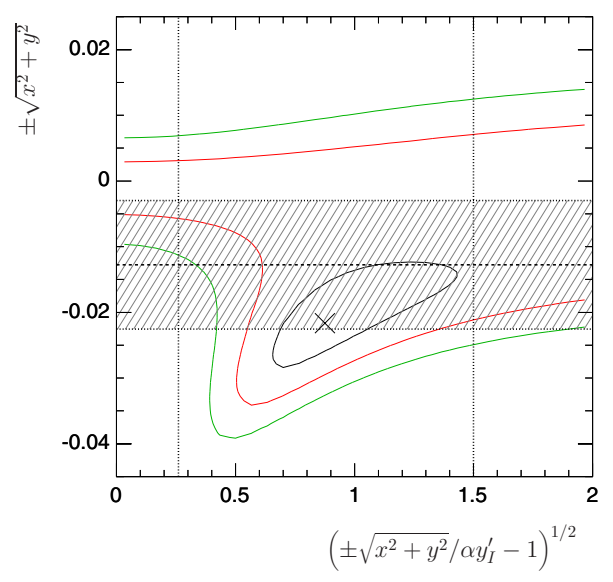

Figure 10.14: Likelihood contours for final data set, where the likelihood is a function of $\left\{a_{1}, a_{2}, a_{3}\right\}$ and the parameter values are constrained to reproduce the number of fitted signal events. The contours indicate $\Delta \log \mathcal{L}=0.5,2.0,4.5$, in black, red, and green, respectively. The cross $(\mathrm{X})$ marks the parameter values that give the maximal likelihood. The vertical lines mark the region of parameter space that, when calculating an expected value of $\pm \sqrt{x^{2}+y^{2}}$, yields symmetric uncertainties which include a null result at the $10 \%$-confidence level. The horizontal dotted line and hatched region designate the corresponding expected value of $\pm \sqrt{x^{2}+y^{2}}$ with $1 \sigma$ confidence intervals. 

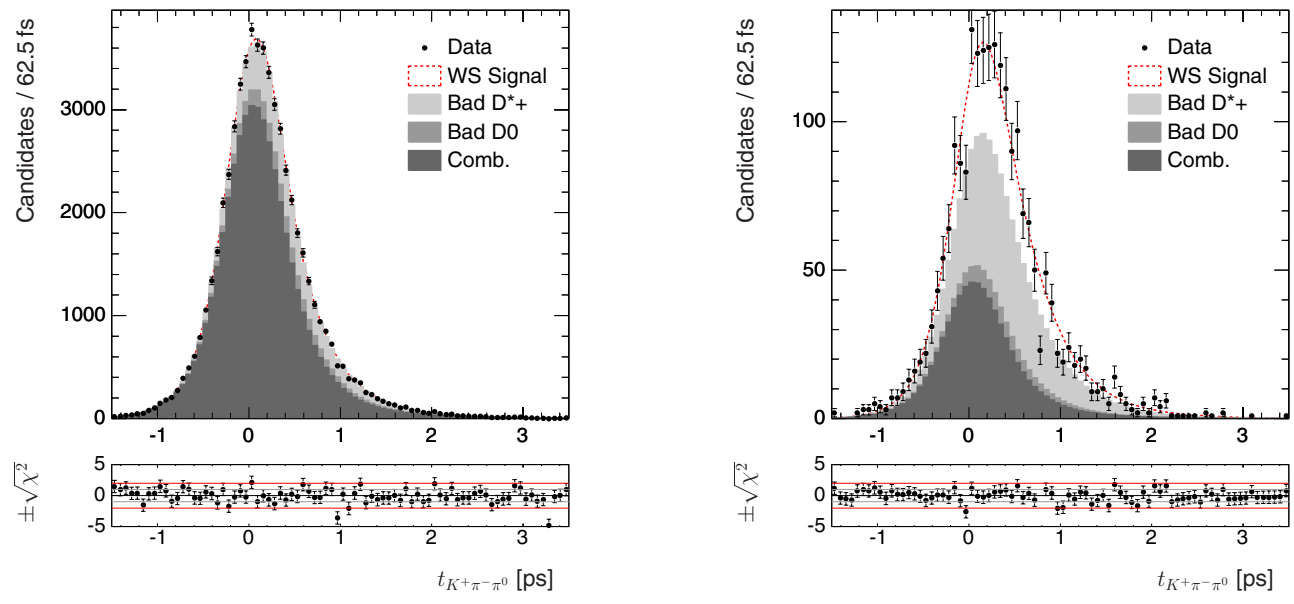

Figure 10.15: Projections of the maximum likelihood fit to the wrong-sign data onto $t_{K \pi \pi^{0}}$ for the entire fit region (left) and for the signal region only (right). The entire fit region is $1.74<m_{K \pi \pi^{0}}<1.98 \mathrm{GeV} / c^{2}$ and $0.13957<\Delta m<0.155 \mathrm{GeV} / c^{2}$; the signal region is $1.85<m_{K \pi \pi^{0}}<1.88 \mathrm{GeV} / c^{2}$ and $0.1444<\Delta m<0.1464 \mathrm{GeV} / c^{2}$.
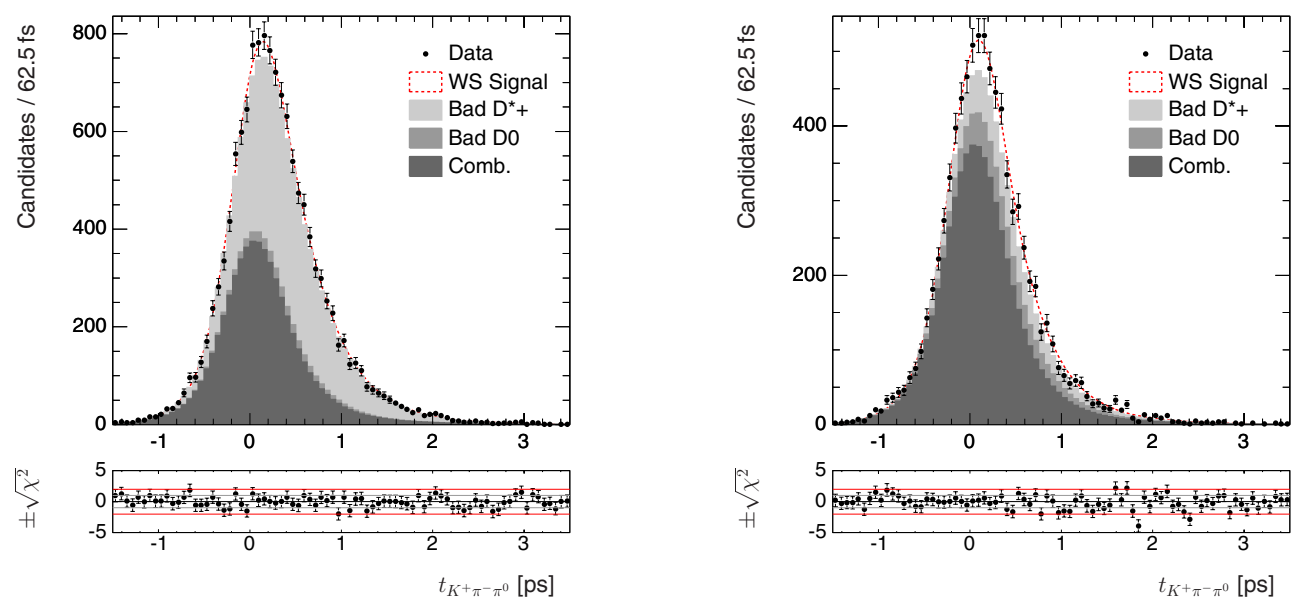

Figure 10.16: Projections of the maximum likelihood fit to the wrong-sign data onto $t_{K \pi \pi^{0}}$ for the $m_{K \pi \pi^{0}}$ signal region (left) and for the $\Delta m$ signal region (right). The $m_{K \pi \pi^{0}}$ signal region is $1.85<m_{K \pi \pi^{0}}<1.88 \mathrm{GeV} / c^{2}$ and $0.13957<\Delta m<$ $0.155 \mathrm{GeV} / c^{2}$; the $\Delta m$ signal region is $1.74<m_{K \pi \pi^{0}}<1.98 \mathrm{GeV} / c^{2}$ and $0.1444<$ $\Delta m<0.1464 \mathrm{GeV} / c^{2}$. 

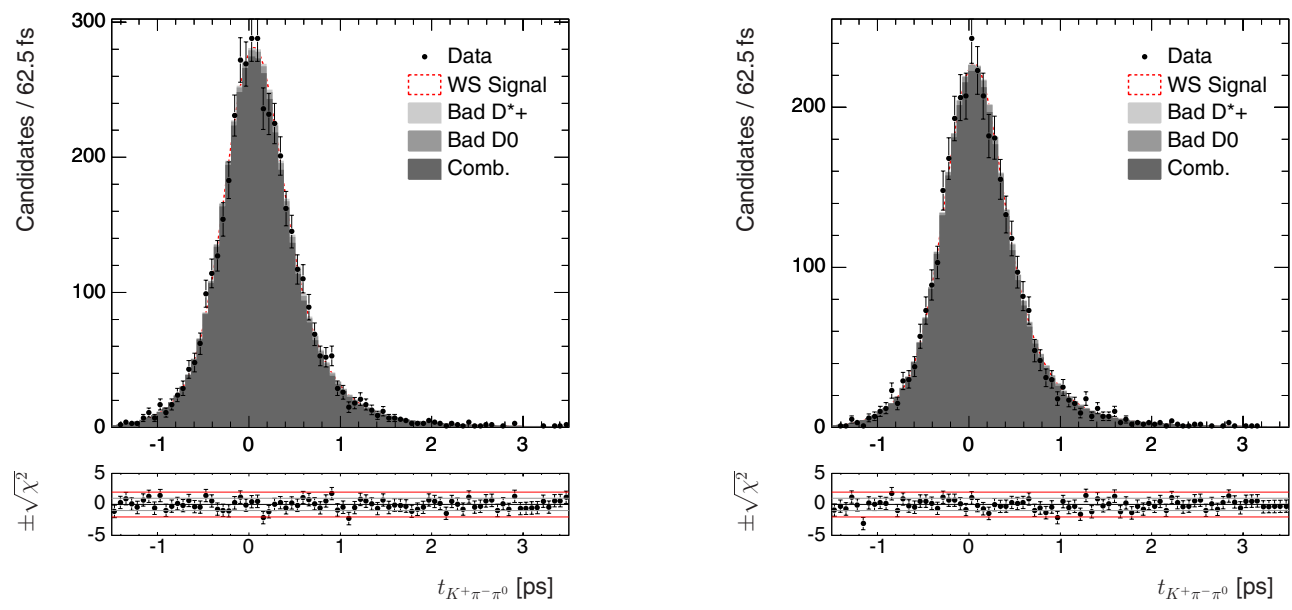

Figure 10.17: Projections of the maximum likelihood fit to the wrong-sign data onto $t_{K \pi \pi^{0}}$ for sideband regions emphasizing the combinatoric background in the lower $m_{K \pi \pi^{0}}$ region (left) and in the upper $m_{K \pi \pi^{0}}$ region (right). The lower $m_{K \pi \pi^{0}}$ region is $1.74<m_{K \pi \pi^{0}}<1.82 \mathrm{GeV} / c^{2}$ and $0.152<\Delta m<0.155 \mathrm{GeV} / c^{2}$; the upper $m_{K \pi \pi^{0}}$ region is $1.91<m_{K \pi \pi^{0}}<1.98 \mathrm{GeV} / c^{2}$ and $0.152<\Delta m<0.155 \mathrm{GeV} / c^{2}$.

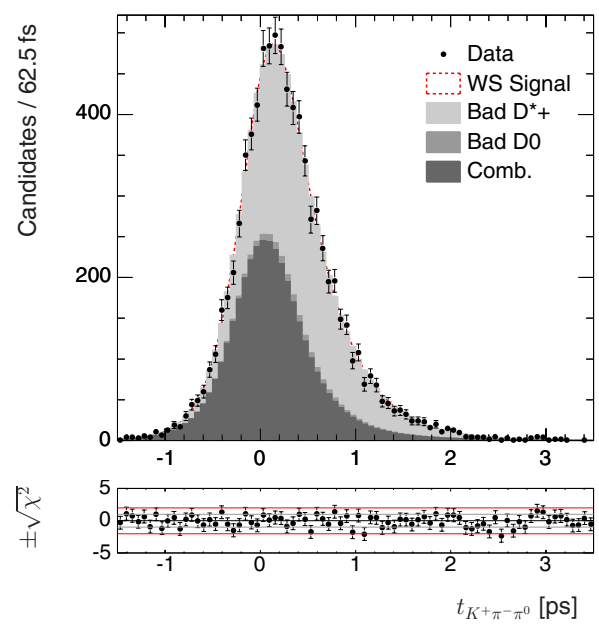

Figure 10.18: Projections of the maximum likelihood fit to the wrong-sign data onto $t_{K \pi \pi^{0}}$ for a sideband region emphasizing the bad- $D^{*+}$ background. The region is $1.85<m_{K \pi \pi^{0}}<1.88 \mathrm{GeV} / c^{2}$ and $0.147<\Delta m<0.155 \mathrm{GeV} / c^{2}$. 

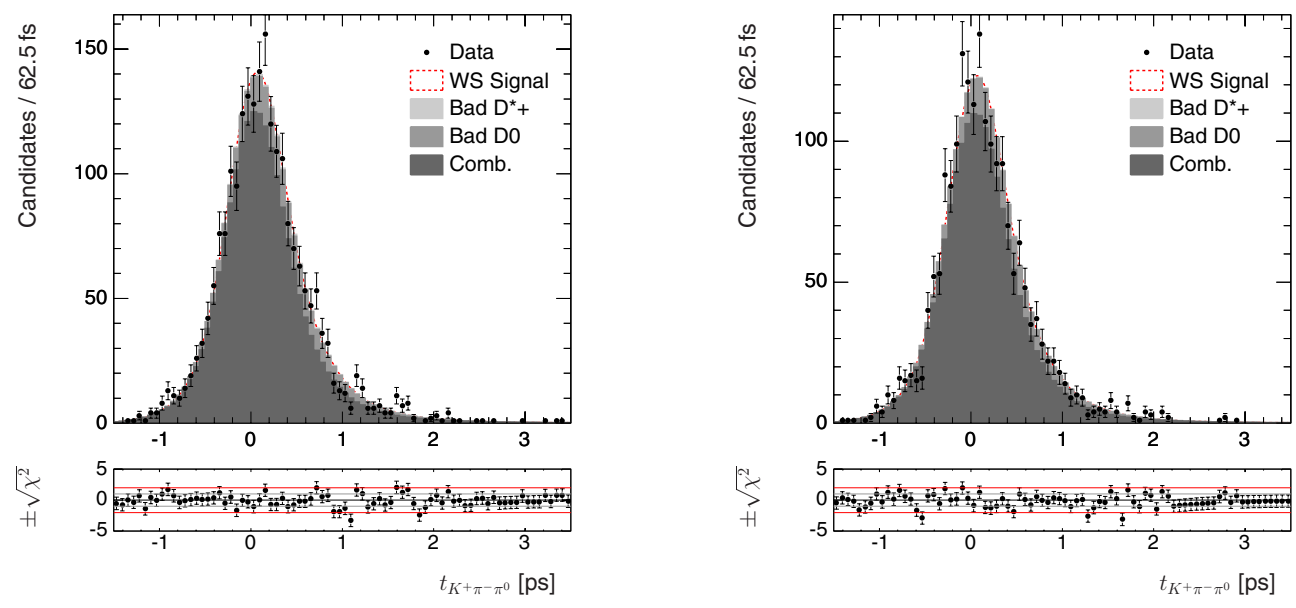

Figure 10.19: Projections of the maximum likelihood fit to the wrong-sign data onto $t_{K \pi \pi^{0}}$ for sideband regions emphasizing the bad- $D^{0}$ background in the lower $m_{K \pi \pi^{0}}$ region (left) and in the upper $m_{K \pi \pi^{0}}$ region (right). The lower $m_{K \pi \pi^{0}}$ region is $1.74<$ $m_{K \pi \pi^{0}}<1.82 \mathrm{GeV} / c^{2}$ and $0.1444<\Delta m<0.1464 \mathrm{GeV} / c^{2}$; the upper $m_{K \pi \pi^{0}}$ region is $1.91<m_{K \pi \pi^{0}}<1.98 \mathrm{GeV} / c^{2}$ and $0.1444<\Delta m<0.1464 \mathrm{GeV} / c^{2}$. 

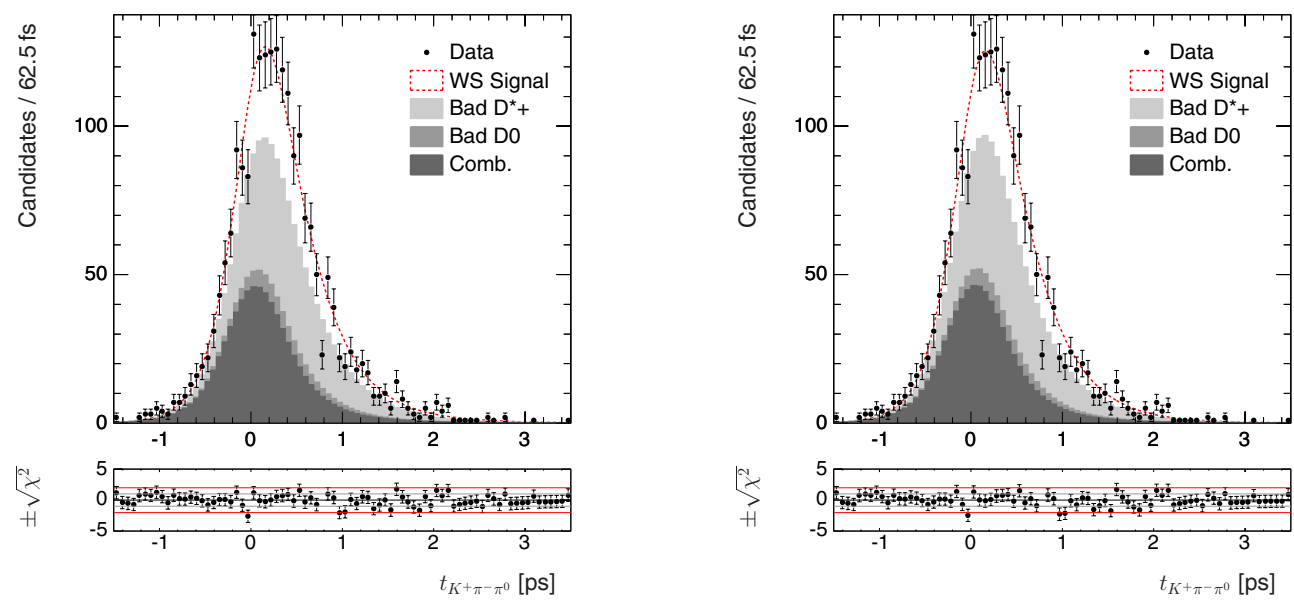

Figure 10.20: Projections of the maximum likelihood fit to the wrong-sign data onto $t_{K \pi \pi^{0}}$ for a signal region, showing the fit with the mixing parameters at the values that maximize the likelihood (left) and with the mixing parameters at the values of the null hypothesis (right). The signal region is $1.85<m_{K \pi \pi^{0}}<1.88 \mathrm{GeV} / c^{2}$ and $0.1444<\Delta m<0.1464 \mathrm{GeV} / c^{2}$. 


\subsection{Conclusions}

The measurement of the integrated $D$ mixing rate $\left(x^{2}+y^{2}\right) / 2$ in this chapter is the most precise to date. The quoted uncertainty is an order of magnitude smaller than previous analyses of the decay-time distribution in $D^{0} \rightarrow K^{+} \pi^{-}$decays. This is due in part to the larger data sample, in part to the accurate and well-tuned maximum likelihood fit, in part to the techniques used to reduce the relative amount of doubly Cabibbo-suppressed decays, and in part to the more robust functional form used in the fit for mixing. A more precise measurement will require more data, a time-dependent Dalitz-plot analysis, or both.

Although the $D$-mixing rate is not very interesting in itself due to inability to predict it accurately, it offers a foothold to obtaining possible indications of Physics beyond the Standard Model. For example, as discussed in Chapter 1, it is generally predicted within the Standard Model that $x \ll y$. With the value of $y \approx 1 \%$, as

measured by BABAR [16], and the value of $\sqrt{x^{2}+y^{2}} \approx 0.013$, as given above, a value $x \approx 0.8 \%$ is calculated, which is about the same magnitude as $y$. Also, the ability to measure the $D$-mixing rate accurately enables one to do $C P$-violation studies, which would indicate new phenomena if $C P$ violation is observed. This result is therefore significant and relevant in the effort to better understand the nature of Particle Physics, and the methods developed for conducting this search may be of general use in the future. 


\section{Appendix A}

\section{Standard Candidate Lists}

\section{A.1 General Track Lists}

\section{A.1.1 GoodTracksVeryLoose}

- $d_{x y}<1.5 \mathrm{~cm}$, where $d_{x y}$ is the distance of closest approach (DOCA) to the beamspot in the transverse plane (relative to the beam axis)

- $d_{z}<10 \mathrm{~cm}$, where $d_{z}$ is the DOCA to the beamspot along the direction of the beam axis 


\section{A.1.2 GoodTracksLoose}

- $d_{x y}<1.5 \mathrm{~cm}$, where $d_{x y}$ is the distance of closest approach (DOCA) to the beamspot in the transverse plane (relative to the beam axis)

- $d_{z}<10 \mathrm{~cm}$, where $d_{z}$ is the DOCA to the beamspot along the direction of the beam axis

- $p_{t}>100 \mathrm{MeV} / c$, where $p_{t}$ is the transverse lab-momentum relative to the beam axis

- At least 12 hits in the drift chamber

\section{A.2 General Neutral Lists}

\section{A.2.1 GoodPhotonLoose}

- $\gamma$ candidates are taken from the CalorNeutral list, which contains single EMC bumps that are not matched with any track

- EMC bump energy $>30 \mathrm{MeV} / c^{2}$

- EMC Lat $<0.8$ (see Table 4.1 for definition) 


\section{A.2.2 pioveryLoose}

- $\gamma$ candidate are taken from the GoodPhotonLoose list, defined above

- $90<m_{\gamma \gamma}<165 \mathrm{MeV} / c^{2}$

- no merged $\pi^{0}$ candidates

\section{A.3 Particle Identification Lists}

\section{A.3.1 KLHTight}

For each track, the selector calculates likelihoods $\mathcal{L}$ for several particle hypotheses. The particle types checked are $K, \pi, p, \mu$, and $e$. The likelihood calculations use information from the SVT and DCH, such as $d E / d x$ and number of hits; information from the EMC, such as $E / P$; and information from the DRC, such as the Cerenkov angle and the number of photons. The selector combines the likelihoods into ratios, and make cuts to select tracks with a given efficiency and fake rate.

Histograms showing the various efficiencies of this selector are found in Figures A.1-A.6. 


\section{A.3.2 KLHVeryTight}

This selector calculate likelihoods as for the KLHTight selector described above, making different cuts on the ratios.

Histograms showing the various efficiencies of this selector are found in Figures A.7-A.12.

\section{A.3.3 piLHTight}

This selector calculate likelihoods as for the KLHTight selector described above, making different cuts on the ratios.

Histograms showing the various efficiencies of this selector are found in Figures A.13-A.18.

\section{A.3.4 pilHVeryTight}

This selector calculate likelihoods as for the KLHTight selector described above, making different cuts on the ratios.

Histograms showing the various efficiencies of this selector are found in Figures A.19-A.24. 


\section{A.3.5 emicroloose}

- $-0.7<\theta_{p}<0.84$, where $\theta_{p}$ is the polar angle of the lab momentum with respect to the beam axis

- $E / p>0.65$, where $E / p$ is the energy deposited in the electromagnetic calorimeter divided by the lab-momentum magnitude

- At least 4 calorimeter crystals in the cluster associated with the track

- $500<d E / d x<1000$, where $d E / d x$ is the ionization energy loss in the drift chamber (values are in arbitrary, detector-specific units)

\section{A.3.6 PidLHElectrons}

For each track, a likelihood is calculated for several particle hypotheses. The likelihood calculation for each particle hypothesis, $\mathcal{L}_{\text {hypo }}$, includes the following quantities:

- $E / p$, the energy deposited in the electromagnetic calorimeter (EMC) divided by the lab-momentum magnitude

- LAT, the lateral moment of the EMC cluster (see Table 4.1 for definition)

- $\Delta \phi$, the angular difference between the centroid of the EMC cluster and the calculated track position at the face of the calorimeter, multiplied by the charge 
of the track

- $d E / d x$, the ionization energy loss in the drift chamber

- $\theta_{C}$, the Cerenkov angle in the DIRC

(if the momentum magnitude $p<2.5 \mathrm{GeV} / c$ )

The total likelihood is defined as

$$
\mathcal{L}=\frac{\mathcal{L}_{e}}{\mathcal{L}_{e}+\mathcal{L}_{\pi}+\mathcal{L}_{K}+\mathcal{L}_{p}}
$$

This selector requires the following criteria:

- $p>300 \mathrm{MeV} / c$, where $p$ is the lab-momentum magnitude

- $-0.74<\theta_{p}<0.84$, where $\theta_{p}$ is the polar angle of the lab momentum with respect to the beam axis.

- $0.65<E / p<1.5$

- At least 5 calorimeter crystals in the cluster associated with the track.

- $500<d E / d x<1000$

- $-0.05<\Delta \phi<0.15$

- At least 6 photons in the DIRC, if at least 6 photons are expected for the track

- $\mathcal{L}>0.98$ 

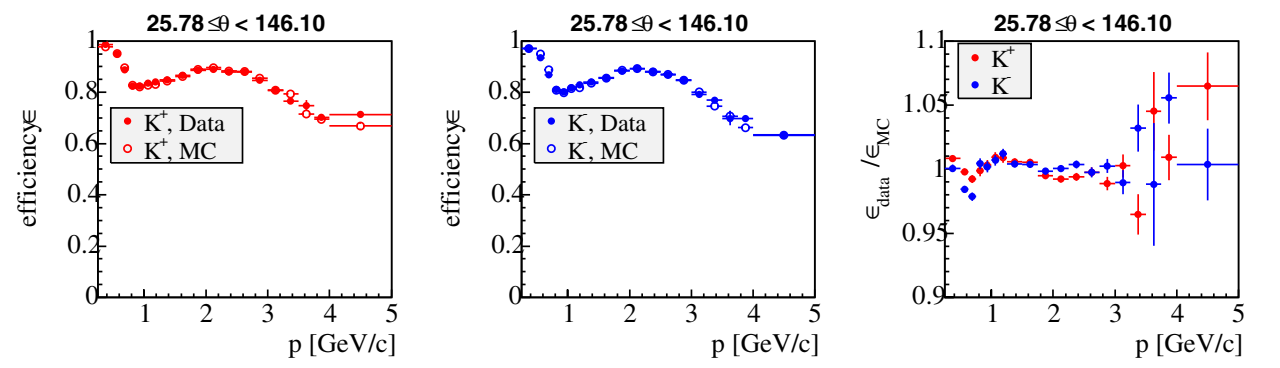

$\overline{\text { Selector : TightLHKaonMicroselection }}$

Dataset : run 4 -r 169

Tables rreated on 1/2/2005 (Data), 1/2/2005 (MC)

Figure A.1: Efficiencies for selecting $K^{ \pm}$s using the KLHTight list in Monte Carlo and data. The histograms show the efficiency as a function of momentum for the entire detector acceptance range. The left shows the efficiency for selecting $K^{+}$, the center shows the efficiency for selecting $K^{-}$, and the right shows the ratio of efficiency in data to that in Monte Carlo.
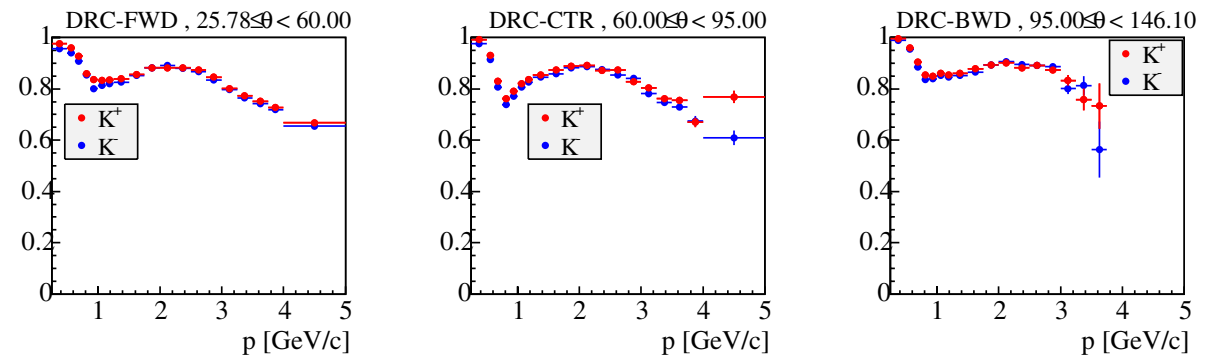

Selector: TightLHKaanMicroselection

Dataset : run4-r16a

Tables crated on 1/2/2005 (Data), 1/22005 (MC)

Figure A.2: Efficiencies for selecting $K^{ \pm}$s using the KLHTight list in data. The histograms show the efficiency for $K^{-}$and $K^{+}$as a function of momentum for the forward section of the detector (left), the center section (center), and the backward section (right) 

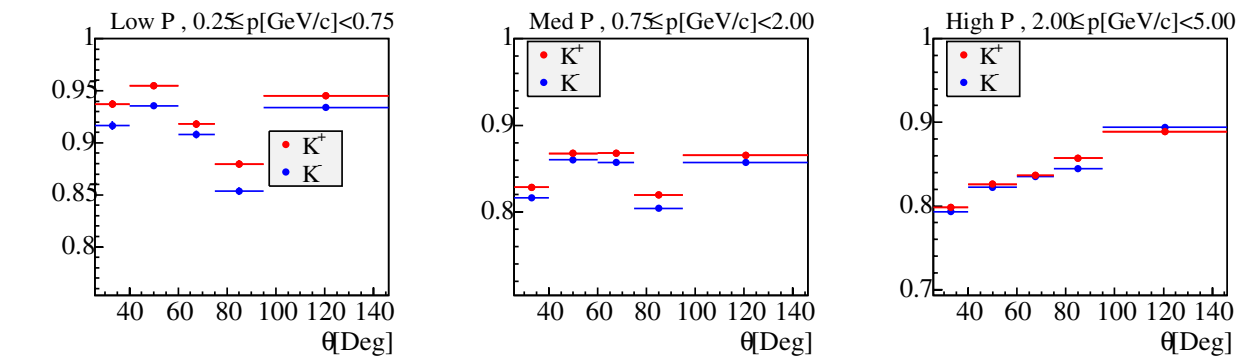

Selector: TightLHKaanMicroselection

Dataset : run4r-r16a

Figure A.3: Efficiencies for selecting $K^{ \pm}$s using the KLHTight list in data. The histograms show the efficiency for $K^{-}$and $K^{+}$as a function of polar angle for the lowmomentum tracks (left), medium-momentum tracks (center), and high-momentum tracks (right)
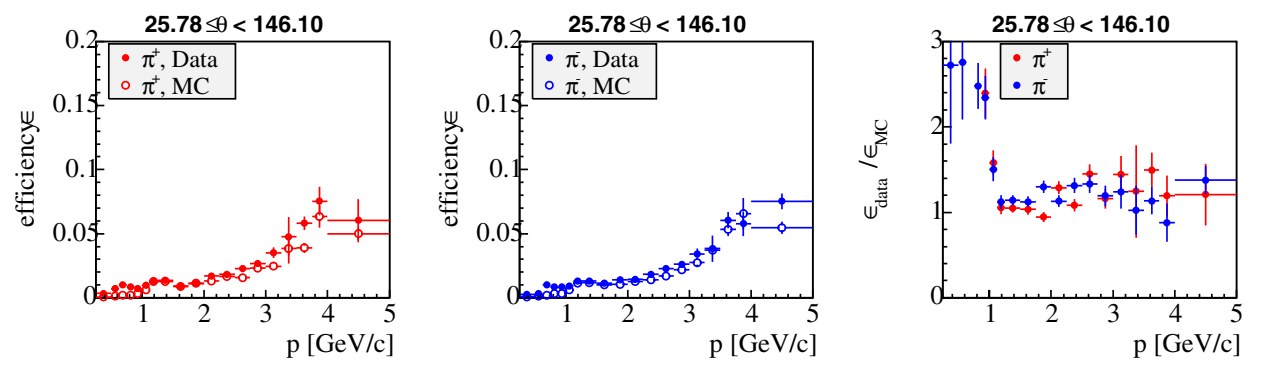

Selector: TightLKKaonMicroselection

Dataset : run4-r16a

Tables created on 1/2/2005 (Data) , 1/2/2005 (MC)

Figure A.4: Efficiencies for selecting $\pi^{ \pm}$s using the KLHTight list in Monte Carlo and data. The histograms show the efficiency as a function of momentum for the entire detector acceptance range. The left shows the efficiency for selecting $\pi^{+}$, the center shows the efficiency for selecting $\pi^{-}$, and the right shows the ratio of efficiency in data to that in Monte Carlo. 

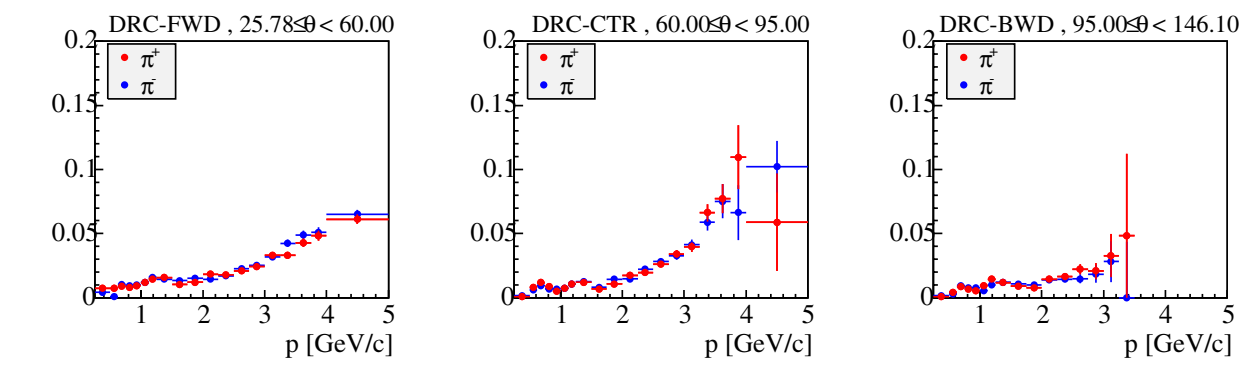

Selector : TightLHKaonMicroSelection

Dataset : run4-r16

Tables created on $1 / 2 / 2005$ (Data), 1/2/2005 (MC)

Figure A.5: Efficiencies for selecting $\pi^{ \pm}$S using the KLHTight list in data. The histograms show the efficiency for $\pi^{-}$and $\pi^{+}$as a function of momentum for the forward section of the detector (left), the center section (center), and the backward section (right)
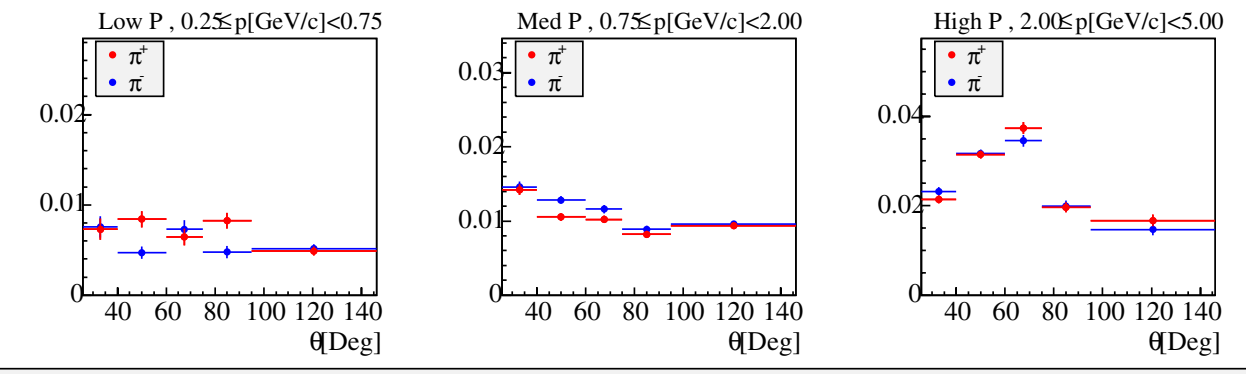

Figure A.6: Efficiencies for selecting $\pi^{ \pm}$S using the KLHTight list in data. The histograms show the efficiency for $\pi^{-}$and $\pi^{+}$as a function of polar angle for the lowmomentum tracks (left), medium-momentum tracks (center), and high-momentum tracks (right) 

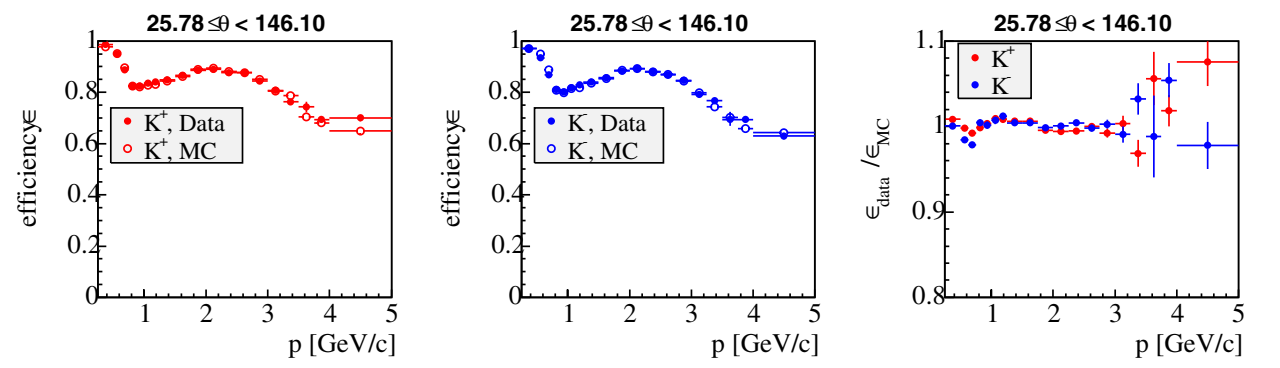

Selector : VeryTightLHKaonMicroSelectio

Dataset : run4-r16

Tables created on 1/2/2005 (Data) , 1/2/2005 (MC)

Figure A.7: Efficiencies for selecting $K^{ \pm} \mathrm{s}$ using the KLHVeryTight list in Monte Carlo and data. The histograms show the efficiency as a function of momentum for the entire detector acceptance range. The left shows the efficiency for selecting $K^{+}$, the center shows the efficiency for selecting $K^{-}$, and the right shows the ratio of efficiency in data to that in Monte Carlo.
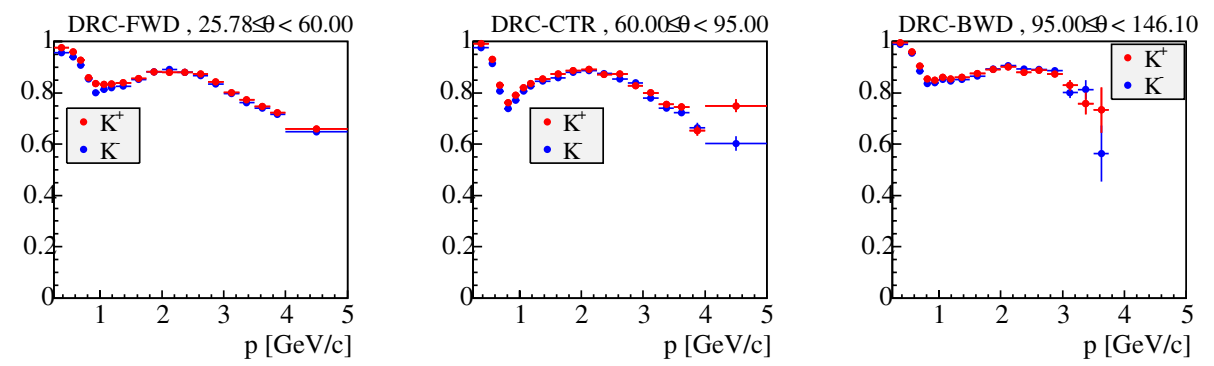

Selector : VeryTightLHKaonMicroSelection

Dataset : run4-r16a

Tables created on 1/2/2005 (Data) , 1/2/2005 (MC)

Figure A.8: Efficiencies for selecting $K^{ \pm}$S using the KLHVeryTight list in data. The histograms show the efficiency for $K^{-}$and $K^{+}$as a function of momentum for the forward section of the detector (left), the center section (center), and the backward section (right) 

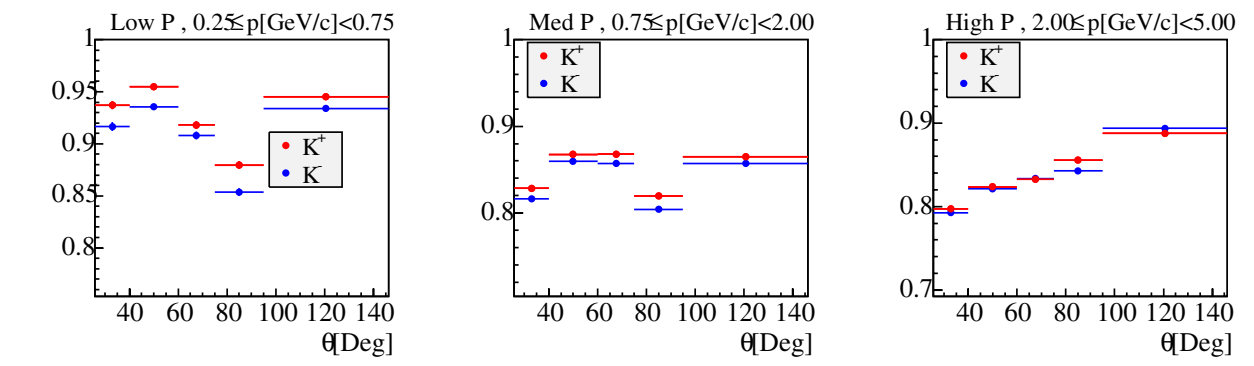

Figure A.9: Efficiencies for selecting $K^{ \pm}$s using the KLHVeryTight list in data. The histograms show the efficiency for $K^{-}$and $K^{+}$as a function of polar angle for the low-momentum tracks (left), medium-momentum tracks (center), and highmomentum tracks (right)
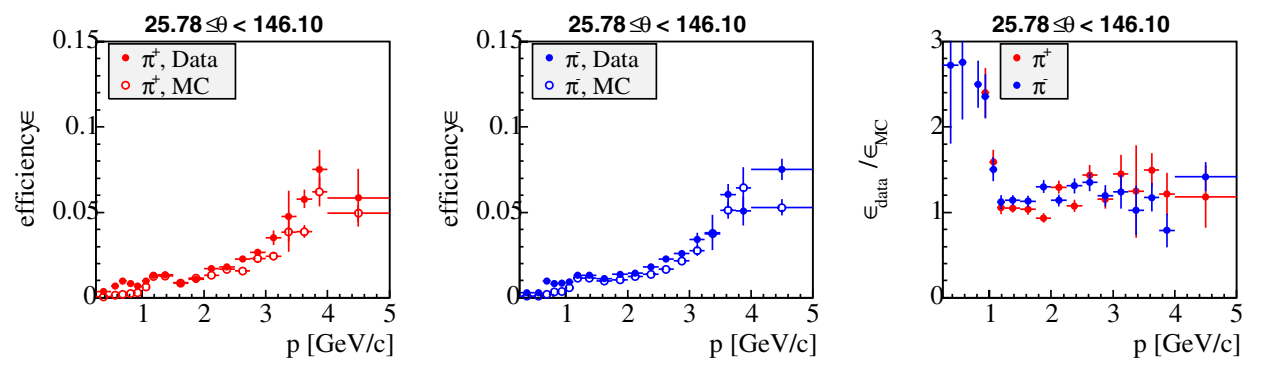

Figure A.10: Efficiencies for selecting $\pi^{ \pm}$s using the KLHVeryTight list in Monte Carlo and data. The histograms show the efficiency as a function of momentum for the entire detector acceptance range. The left shows the efficiency for selecting $\pi^{+}$, the center shows the efficiency for selecting $\pi^{-}$, and the right shows the ratio of efficiency in data to that in Monte Carlo. 

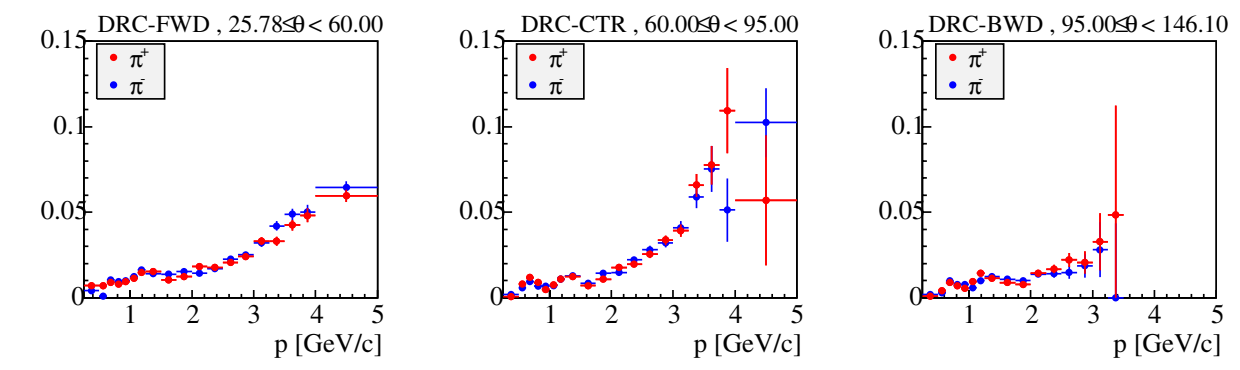

Selector: VeryTightLHKaonMiroselection

Dataset : run4-r16a

Tables created on $1 / 2 / 2005$ (Date) $1 / 2 / 2005(\mathrm{MC})$

Figure A.11: Efficiencies for selecting $\pi^{ \pm}$s using the KLHVeryTight list in data. The histograms show the efficiency for $\pi^{-}$and $\pi^{+}$as a function of momentum for the forward section of the detector (left), the center section (center), and the backward section (right)
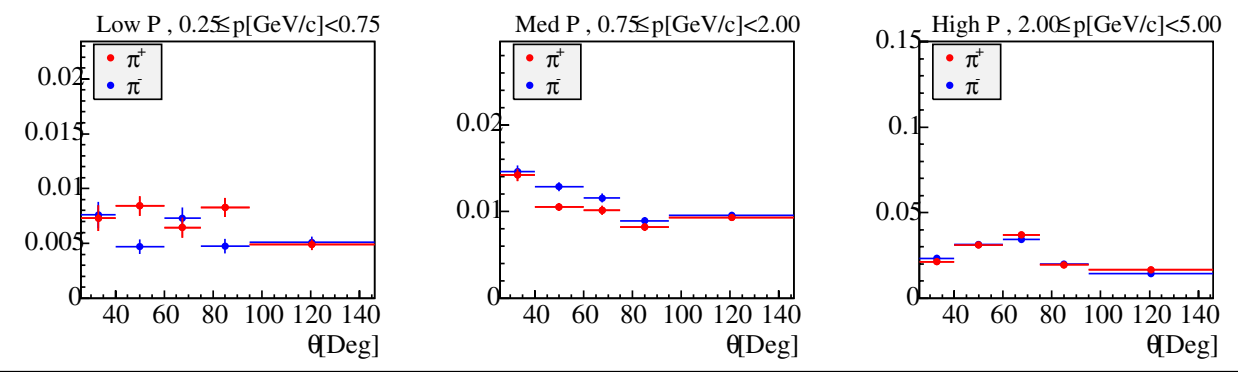

Selector: VeryTighLLHKaonMicroSelection

Dataset : run4-r16a

Tables created on 1/2/2005 (Data)

Figure A.12: Efficiencies for selecting $\pi^{ \pm}$s using the KLHVeryTight list in data. The histograms show the efficiency for $\pi^{-}$and $\pi^{+}$as a function of polar angle for the low-momentum tracks (left), medium-momentum tracks (center), and highmomentum tracks (right) 

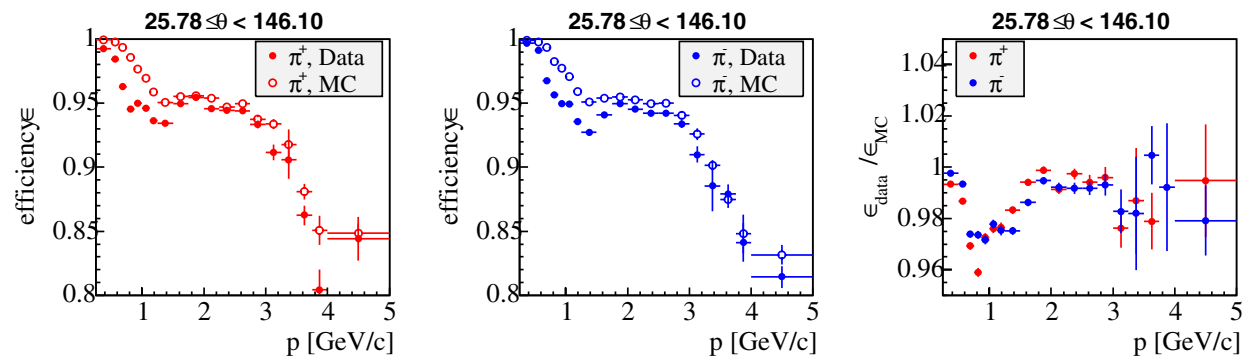

Selector : TightLHPionMicroSelection

Dataset : run4-r16

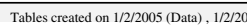

Figure A.13: Efficiencies for selecting $\pi^{ \pm} \mathrm{S}$ using the piLHTight list in Monte Carlo and data. The histograms show the efficiency as a function of momentum for the entire detector acceptance range. The left shows the efficiency for selecting $\pi^{+}$, the center shows the efficiency for selecting $\pi^{-}$, and the right shows the ratio of efficiency in data to that in Monte Carlo.
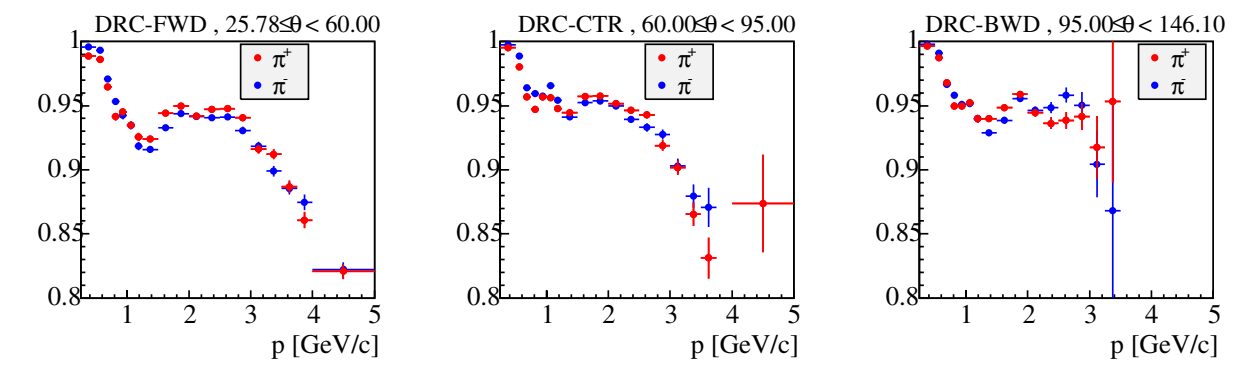

Selector : TightLHPionMicroSelection

Dataset: run4-r16a

Figure A.14: Efficiencies for selecting $\pi^{ \pm}$S using the piLHTight list in data. The histograms show the efficiency for $\pi^{-}$and $\pi^{+}$as a function of momentum for the forward section of the detector (left), the center section (center), and the backward section (right) 

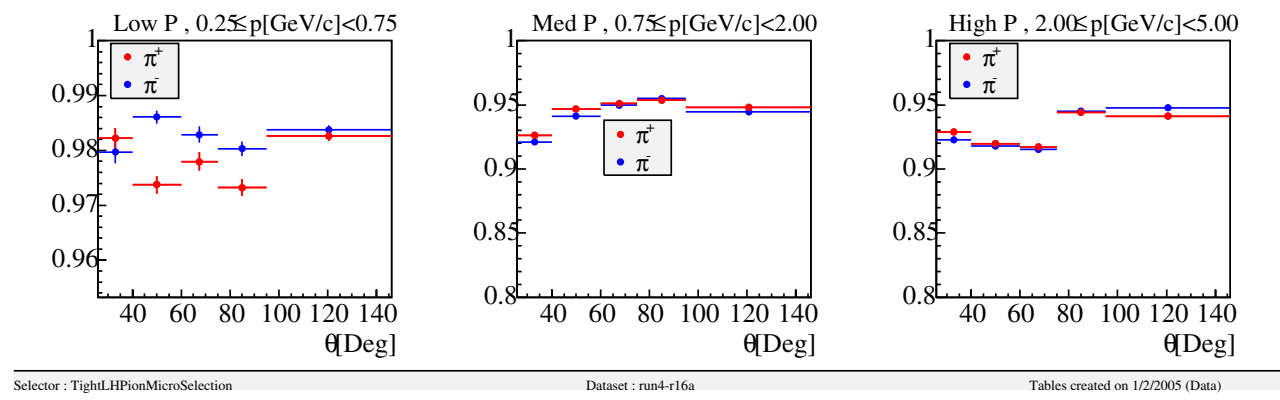

Figure A.15: Efficiencies for selecting $\pi^{ \pm}$s using the piLHTight list in data. The histograms show the efficiency for $\pi^{-}$and $\pi^{+}$as a function of polar angle for the lowmomentum tracks (left), medium-momentum tracks (center), and high-momentum tracks (right)
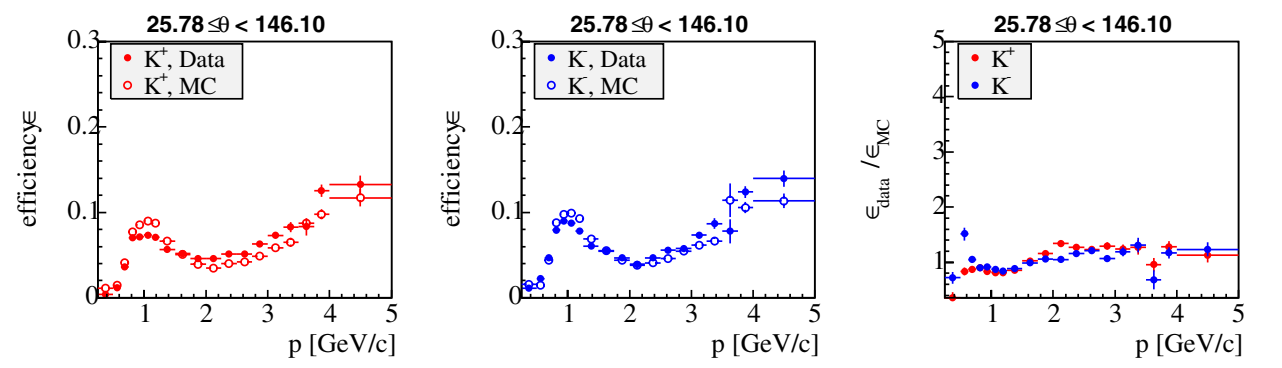

Selector : TightLHPionMicroSelection

Dataset : run4-r16

Tables created on 1/2/2005 (Data) , 1/2/2005 (MC)

Figure A.16: Efficiencies for selecting $K^{ \pm}$s using the piLHTight list in Monte Carlo and data. The histograms show the efficiency as a function of momentum for the entire detector acceptance range. The left shows the efficiency for selecting $K^{+}$, the center shows the efficiency for selecting $K^{-}$, and the right shows the ratio of efficiency in data to that in Monte Carlo. 

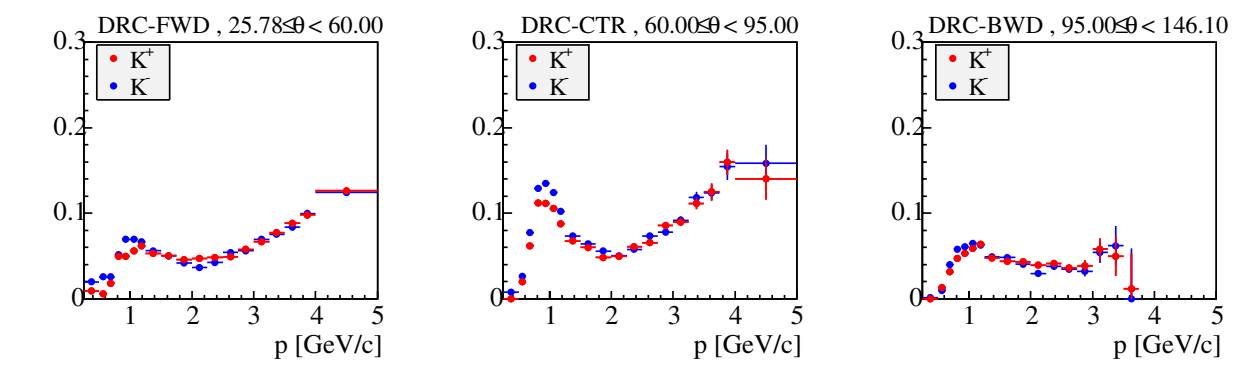

Selector : TightLHPionMicroSelection

Dataset : run4-r16a

Tables created on 1/2/2005 (Data), 1/2/2005 (MC)

Figure A.17: Efficiencies for selecting $K^{ \pm}$s using the piLHTight list in data. The histograms show the efficiency for $K^{-}$and $K^{+}$as a function of momentum for the forward section of the detector (left), the center section (center), and the backward section (right)
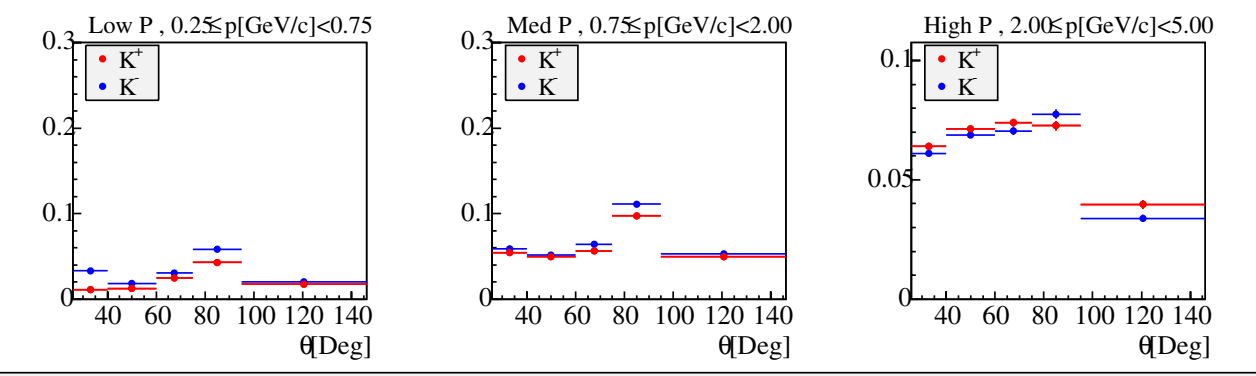

Figure A.18: Efficiencies for selecting $K^{ \pm}$s using the piLHTight list in data. The histograms show the efficiency for $K^{-}$and $K^{+}$as a function of polar angle for the low-momentum tracks (left), medium-momentum tracks (center), and highmomentum tracks (right) 

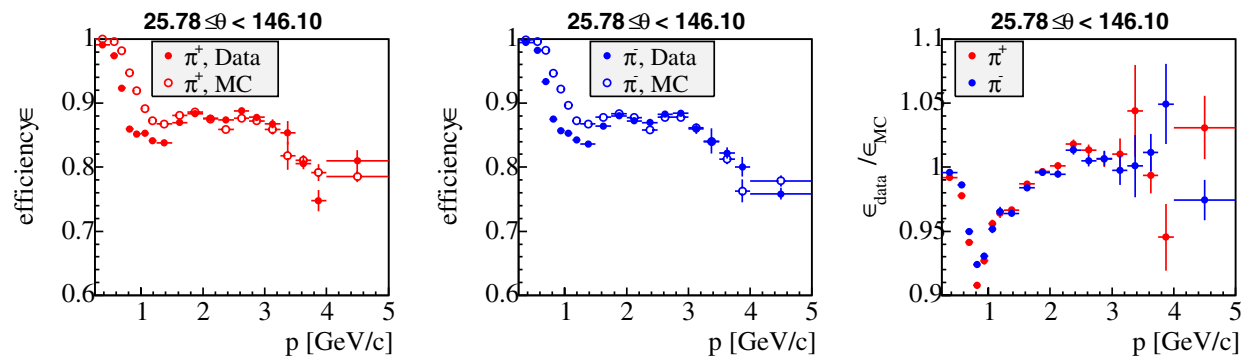

$\overline{\text { Selector : VeryTightLHPionMicroSelection }}$

Dataset : run4-r16

Figure A.19: Efficiencies for selecting $\pi^{ \pm}$s using the piLHVeryTight list in Monte Carlo and data. The histograms show the efficiency as a function of momentum for the entire detector acceptance range. The left shows the efficiency for selecting $\pi^{+}$, the center shows the efficiency for selecting $\pi^{-}$, and the right shows the ratio of efficiency in data to that in Monte Carlo.
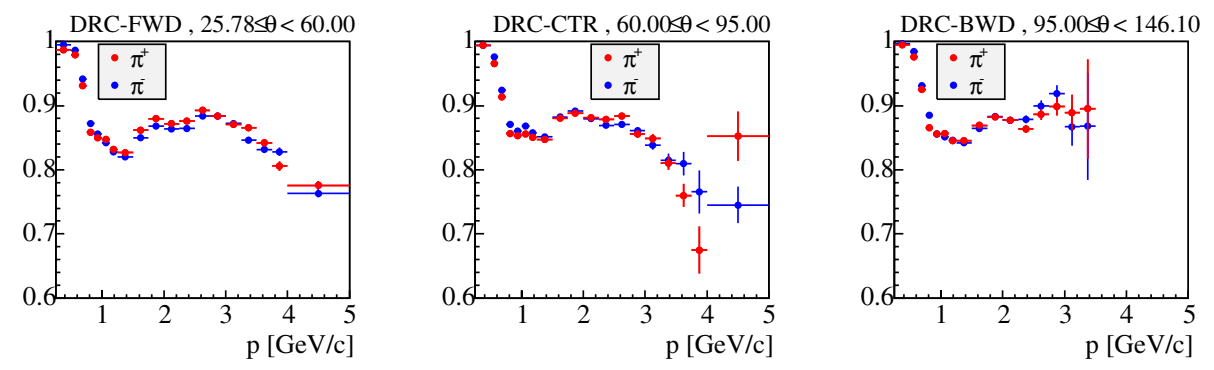

Selector: VeryTightLHPionMicroselection $\quad$ Dataset : run4-ris

Tables created on 1/2/2005 (Data) , 1/2/2005 (MC)

Figure A.20: Efficiencies for selecting $\pi^{ \pm}$s using the piLHVeryTight list in data. The histograms show the efficiency for $\pi^{-}$and $\pi^{+}$as a function of momentum for the forward section of the detector (left), the center section (center), and the backward section (right) 

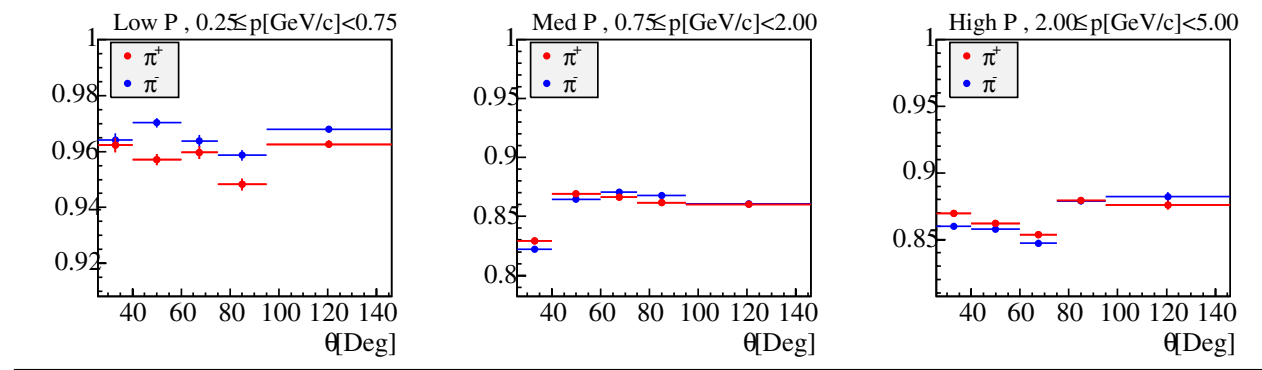

Figure A.21: Efficiencies for selecting $\pi^{ \pm}$s using the piLHVeryTight list in data. The histograms show the efficiency for $\pi^{-}$and $\pi^{+}$as a function of polar angle for the low-momentum tracks (left), medium-momentum tracks (center), and highmomentum tracks (right)
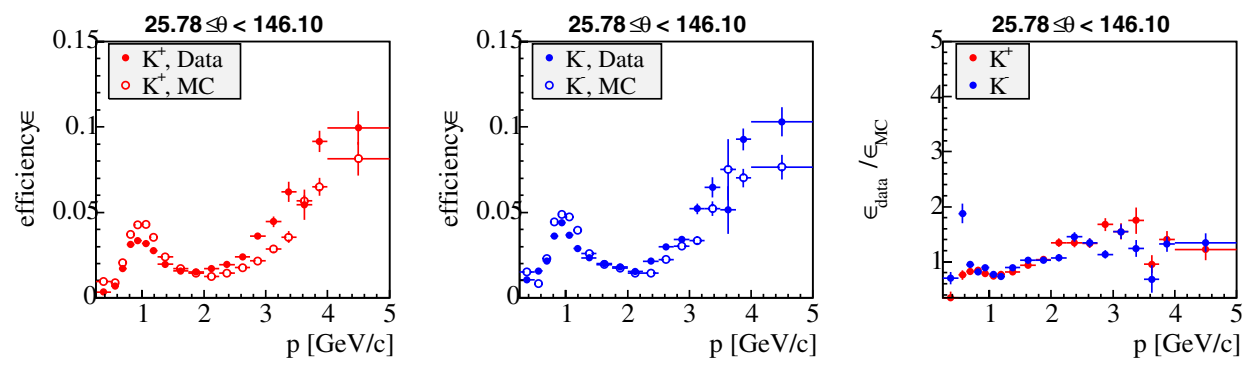

Figure A.22: Efficiencies for selecting $K^{ \pm}$s using the piLHVerYTight list in Monte Carlo and data. The histograms show the efficiency as a function of momentum for the entire detector acceptance range. The left shows the efficiency for selecting $K^{+}$, the center shows the efficiency for selecting $K^{-}$, and the right shows the ratio of efficiency in data to that in Monte Carlo. 

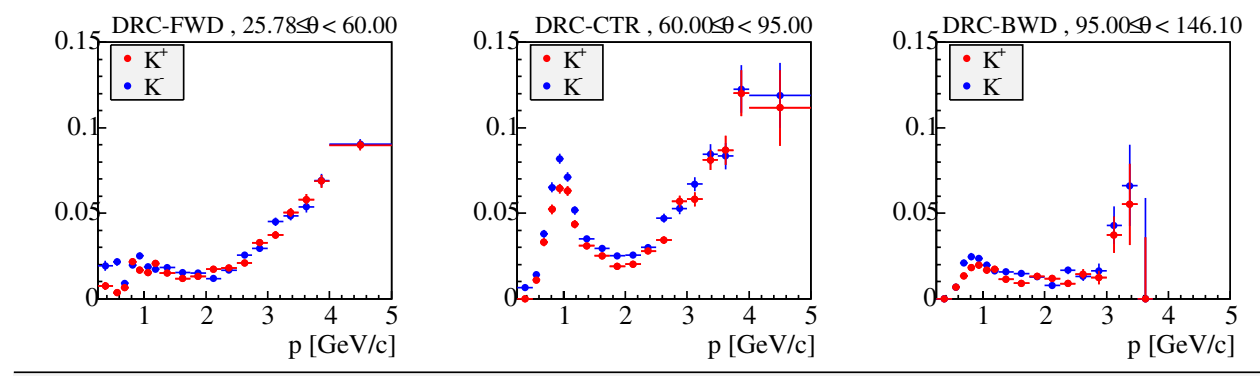

Figure A.23: Efficiencies for selecting $K^{ \pm}$s using the piLHVeryTight list in data. The histograms show the efficiency for $K^{-}$and $K^{+}$as a function of momentum for the forward section of the detector (left), the center section (center), and the backward section (right)
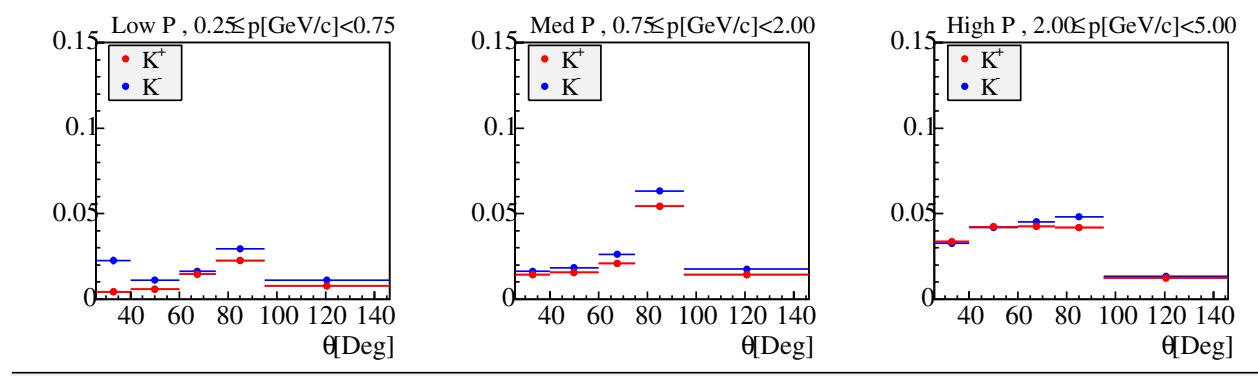

Figure A.24: Efficiencies for selecting $K^{ \pm}$s using the piLHVeryTight list in data. The histograms show the efficiency for $K^{-}$and $K^{+}$as a function of polar angle for the low-momentum tracks (left), medium-momentum tracks (center), and highmomentum tracks (right) 


\section{Bibliography}

[1] G. Burdman and I. Shipsey, Ann. Rev. Nucl. Part. Sci. 53, 431 (2003), [hep$\mathrm{ph} / 0310076]$.

[2] A. Datta and D. Kumbhakar, Z. Phys. C27, 515 (1985).

[3] L. Wolfenstein, Phys. Lett. B164, 170 (1985).

[4] J. F. Donoghue, E. Golowich, B. R. Holstein and J. Trampetic, Phys. Rev. D33, 179 (1986).

[5] A. F. Falk, Y. Grossman, Z. Ligeti and A. A. Petrov, Phys. Rev. D65, 054034 (2002), [hep-ph/0110317].

[6] H. Georgi, Phys. Lett. B297, 353 (1992), [hep-ph/9209291].

[7] T. Ohl, G. Ricciardi and E. H. Simmons, Nucl. Phys. B403, 605 (1993), [hep$\mathrm{ph} / 9301212]$.

[8] I. I. Y. Bigi and N. G. Uraltsev, Nucl. Phys. B592, 92 (2001), [hep-ph/0005089]. 
[9] S. Bergmann, Y. Grossman, Z. Ligeti, Y. Nir and A. A. Petrov, Phys. Lett. B486, 418 (2000), [hep-ph/0005181].

[10] BABAR, B. Aubert et al., Phys. Rev. Lett. 91, 171801 (2003), [hepex/0304007].

[11] BELLE, K. Abe et al., Phys. Rev. Lett. 94, 071801 (2005), [hep-ex/0408125].

[12] CLEO, R. Godang et al., Phys. Rev. Lett. 84, 5038 (2000), [hep-ex/0001060].

[13] M. Gronau, Y. Grossman and J. L. Rosner, Phys. Lett. B508, 37 (2001), [hep$\mathrm{ph} / 0103110]$.

[14] E791, E. M. Aitala et al., Phys. Rev. Lett. 83, 32 (1999), [hep-ex/9903012].

[15] FOCUS, J. M. Link et al., Phys. Lett. B485, 62 (2000), [hep-ex/0004034].

[16] BABAR, B. Aubert et al., Phys. Rev. Lett. 91, 121801 (2003), [hepex/0306003].

[17] E791, E. M. Aitala et al., Phys. Rev. Lett. 77, 2384 (1996), [hep-ex/9606016].

[18] BABAR, B. Aubert et al., Phys. Rev. D70, 091102 (2004), [hep-ex/0408066].

[19] CLEO, G. Brandenburg et al., Phys. Rev. Lett. 87, 071802 (2001), [hepex/0105002].

[20] Belle, X. C. Tian et al., hep-ex/0507071. 
[21] BABAR, B. Aubert et al., Nucl. Instrum. Meth. A479, 1 (2002), [hepex/0105044].

[22] M. Pivk and F. R. Le Diberder, arXiv:physics/0402083.

[23] Particle Data Group, S. Eidelman et al., Phys. Lett. B592, 1 (2004).

[24] CLEO, S. Kopp et al., Phys. Rev. D63, 092001 (2001), [hep-ex/0011065]. 